\title{
Economics of Ramping Rate Restrictions at Hydro Power Plants: Balancing Profitability and Environmental Concerns
}

Shilei Niu

\author{
A thesis \\ presented to the University of Waterloo \\ in fulfillment of the \\ thesis requirement for the degree of \\ Doctor of Philosophy \\ in \\ Applied Economics
}

Waterloo, Ontario, Canada, 2014

(c) Shilei Niu 2014 


\section{Author's Declaration}

I hereby declare that I am the sole author of this thesis. This is a true copy of the thesis, including any required final revisions, as accepted by my examiners.

I understand that my thesis may be made electronically available to the public. 


\begin{abstract}
This thesis consists of three essays on the economics of ramping rate restrictions at hydro power plants. The first essay examines the impact of ramping rate restrictions imposed on hydro operations to protect aquatic ecosystems. A dynamic optimization model of the profit maximizing decisions of a hydro operator is solved for various restrictions on water flow, using data for a representative hydro operation in Ontario. Profits are negatively affected, but for a range of restrictions the impact is not large. Ramping restrictions cause a redistribution of hydro production over a given day, which can result in an increase in total hydro power produced. This affects the need for power from other sources with consequent environmental impacts.
\end{abstract}

The second essay uses a real options approach to study the impact of ramping rate restrictions on hydro power plants. We consider the effect on profits from electricity generation in order to inform policy decisions about ramping rate restrictions. A novelty of the essay is in examining the optimal operation of a prototype hydro power plant with electricity prices modelled as a regime switching process. We show that profits are negatively affected by ramping restrictions. Interestingly for a large range of restrictions, profit is not sensitive to ramping restrictions. The results point to the importance of accurately modelling electricity prices in gauging the trade offs involved in imposing restrictions on hydro operators which may hinder their ability to respond to volatile electricity prices and meet peak demands.

The third essay investigates the impact of ramping rate restrictions on hydro power plants using a three regimes model with multiple jump sizes. We consider how the multiple jump sizes among these three regimes affect the impact of ramping restrictions on the prototype hydro power plant. The numerical experiments provide further evidence that ramping restrictions have a larger impact when the expected variation in price is increased such as through an increase in the jump size which makes it desirable to change water release rates relatively frequently.

In both non-stochastic and stochastic settings, these three essays have highly consistent results on the impact of ramping restrictions on the hydro station's profit. We observe 
profits are significantly affected (by less than $7 \%$ in essay one, by less than $10 \%$ in essay two, and by less than $9 \%$ in essay three) in the case of the most severe ramping constraints, but we also observe a range of less severe ramping restrictions over which profits are not substantially affected (by less than $2 \%$ in essay one, by less than $3 \%$ in essay two, and by less than $2 \%$ in essay three). Results from this thesis should facilitate the implementation of ramping rate restrictions for environmental and economic benefits. 


\section{Acknowledgements}

First and foremost, my sincere gratitude goes to my supervisor, Professor Margaret Insley. This thesis would not have been possible without her continuous help and guidance. Thanks must also be extended to my co-supervisor Professor Tony Wirjanto for his considerable support and encouragement at various stages of my Ph.D. study. Thanks also to my committee members: Professor Pierre Chaussé, Professor Ranjini Jha, Professor Horatiu Rus as well as my external examiner Professor Jean-Daniel Saphores. Thank you for taking the time to read my thesis and provide me with useful comments. I would like to express my thanks to all the people who have helped me during my time at Waterloo.

I wish to acknowledge the Ontario Centres of Excellence (OCE) for the financial support. I also would like to thank the Canadian Association for Energy Economics and the Institute for Quantitative Finance and Insurance at the University of Waterloo for their financial support.

I would like to greatly thank my parents for their continuous support and encouragement. Finally, I would like to thank my wife for her patience and understanding over the past two years. 


\section{Table of Contents}

List of Tables $\quad$ x

List of Figures $\quad$ xii

1 Introduction $\quad 1$

2 On the Economics of Ramping Rate Restrictions at Hydro Power Plants: $\begin{array}{ll}\text { Balancing Profitability and Environmental Costs } & 8\end{array}$

2.1 Introduction . . . . . . . . . . . . . . . . . . 8

2.2 Literature Review . . . . . . . . . . . . . . . . . . . . 10

2.3 Assumptions and Model Formulation . . . . . . . . . . . . . . . . . 14

2.4 The Optimization Problem . . . . . . . . . . . . . . . . 20

2.5 Data Description . . . . . . . . . . . . . . . . . . 24

2.6 Modeling Hydropower Generation . . . . . . . . . . . . . . . . 26

2.7 Empirical Analysis . . . . . . . . . . . . . . . . . . . . . . . . 27

2.7.1 Baseline Optimization . . . . . . . . . . . . . . 30

2.7.2 Optimization with Release Rate Constraints . . . . . . . . . . 33

2.7.3 Optimization with Ramping Rate Constraints . . . . . . . . . . 36 
2.7.4 Comparing the Three Optimization Scenarios . . . . . . . . . 36

2.8 Including the Environmental Impact of Changes in Thermal Generation . . 45

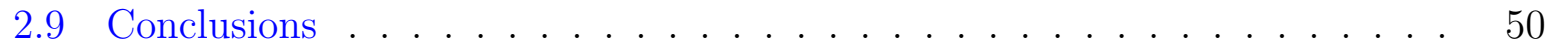

3 An Options Pricing Approach to Ramping Rate Restrictions at Hydro Power Plants $\quad 52$

3.1 Introduction . . . . . . . . . . . . . . . . . . . . 52

3.2 Related Literature . . . . . . . . . . . . . . . . . . . 56

3.3 Electricity Price Models _. . . . . . . . . . . . . . . . . . . . . . . . 59

3.4 Modelling Optimal Hydro Operations . . . . . . . . . . . . . . . . . . . 61

3.4.1 Physical and Environmental Constraints . . . . . . . . . . . . . 61

3.4.2 The Optimization Problem . . . . . . . . . . . . . . . . . . 64

3.5 Hamilton Jacobi Bellman Equations . . . . . . . . . . . . . . . . . . 65

3.5.1 HJB-PDE for the Regime Switching Model . . . . . . . . . . . 65

3.5 .2 Boundary Conditions . . . . . . . . . . . . . . . . . 67

3.6 Specification of the Empirical Example . . . . . . . . . . . . . . . . 69

3.6.1 Description of the Hydro Plant . . . . . . . . . . . . . . . 69

3.6.2 Specification of the Regime Switching Price Process . . . . . . . 71

3.7 Empirical Results . . . . . . . . . . . . . . . . . . . . . . . 75

3.7 .1 Base Case . . . . . . . . . . . . . . . . . . . . . 75

3.7.2 Impact of Changing Ramping Restrictions . . . . . . . . . . . 82

3.7 .3 Sensitivity Analysis . . . . . . . . . . . . . . . . . . 88

3.8 Conclusions . . . . . . . . . . . . . . . . . . . . . . . . . . . . . 91 
4 An Options Pricing Approach to Ramping Rate Restrictions at Hydro Power Plants: An Extension to Three Price Regimes 94

4.1 Introduction . . . . . . . . . . . . . . . . . . . . . . . . . . . 94

4.2 Empirical Analysis . . . . . . . . . . . . . . . . . . . 96

4.3 Case I: Large Jump Size from the Base Regime to the Second Spike Regime 98 4.3 .1 Base Case for Case I . . . . . . . . . . . . . . . . . . . . . . 98

4.3.2 Impact of Changing Ramping Restrictions for Case I . . . . . . . 101

4.4 Case II: Small Jump Size from the Base Regime to the Second Spike Regime 109

4.5 Conclusions . . . . . . . . . . . . . . . . . . . . . . . . . 118

5 Conclusions $\quad 119$

$\begin{array}{lr}\text { APPENDICES } & 124\end{array}$

$\begin{array}{ll}\text { A Appendix to Chapter } 3 & 125\end{array}$

A.1 Derivation and Numerical Algorithms of HJB-PDE . . . . . . . . . . 125

A.1.1 Derivation of HJB-PDE . . . . . . . . . . . . . . . 125

A.1.2 Numerical Algorithms . . . . . . . . . . . . . . . . . . 130

A.1.3 Numerical Scheme for Pricing Equations . . . . . . . . . . . . . . . 131

A.1.4 Solving the Local Optimization Problems . . . . . . . . . . . . . 134

A.1.5 Matrix Form of the Discrete Equations . . . . . . . . . . . 135

A.1.6 Solution Algorithms . . . . . . . . . . . . . . . . . . . . . 137

A.1.7 Derivation of Equation (A.10) for Interior Prices . . . . . . . . . 140

A.1.8 Derivation of Equation (A.10) for the Price at the Lower Bound . . 144

A.1.9 Derivation of Equation (A.10) for the Price at the Upper Bound . . 145

A.2 Tables for the Sensitivity Analyses _ . . . . . . . . . . . . . 146 
References 


\section{List of Tables}

2.1 Parameter Values Used in the Empirical Examples . . . . . . . . . . . . . 29

2.2 Baseline Experiment . . . . . . . . . . . . . . . . . . 31

2.3 Case Including Minimum and Maximum Release Constraints . . . . . . . . 34

2.4 Case Including Up-ramping and Down-ramping Constraints . . . . . . . . 37

2.5 Comparing Profits under Various Equal Levels of Up-ramping and Downramping Constraints .................... . . 41

3.1 Parameter Values for the Abitibi Canyon Station . . . . . . . . . . . . . 71

3.2 Parameter Values Estimated by Janczura and Weron [2009] . . . . . . . . . 72

3.3 Parameter Values for the Regime Switching Model . . . . . . . . . . . . . . 74

3.4 Numerical Results Under Various Ramping Restrictions . . . . . . . . . . . 84

4.1 Parameter Values for the Regime Switching Model (Three Price Regimes) . 97

4.2 Numerical Results Under Various Ramping Restrictions (Large Jump Size from the Base Regime to the Second Spike Regime) . . . . . . . . . . . . 107

4.3 Numerical Results Under Various Ramping Restrictions (Small Jump Size from the Base Regime to the Second Spike Regime) . . . . . . . . . . . . . 116

A.1 Numerical Results with Different Mean Reverting Rate . . . . . . . . . . . 147 
A.2 Numerical Results with Different Transition Probabilities . . . . . . . . . . 148

A.3 Numerical Results with Different Volatilities . . . . . . . . . . . . . . . 149

A.4 Numerical Results with Different Base Regime Mean . . . . . . . . . . . . 150

A.5 Numerical Results with Different Production Cost . . . . . . . . . . . . . . 151

A.6 Summary of Results from the Sensitivity Analysis . . . . . . . . . . . . 152

A.7 Base Regime as Single Regime for the Regime Switching Model . . . . . . 153

A.8 Base Regime as Single Regime for the Regime Switching Model with Different Mean Reverting Rate . . . . . . . . . . . . . . . . . . . . . 153

A.9 Base Regime as Single Regime for the Regime Switching Model with Different Volatilities . . . . . . . . . . . . . . . . . . . 154

A.10 Base Regime as Single Regime for the Regime Switching Model with Different Production Cost . . . . . . . . . . . . . . . . . . . . 154 


\section{List of Figures}

2.1 Hydro and Thermal Power Production: Baseline Experiment . . . . . . . . 32

2.2 Hydro and Thermal Power Production: Experiment with Minimum and Maximum Release Constraints . . . . . . . . . . . . . . . . . . . . . . . . . 35

2.3 Hydro and Thermal Power Production: Experiment with Up-ramping and Down-ramping Constraints . . . . . . . . . . . . . . . . . . 38

2.4 Comparing Water Release Rates for Hydro Production . . . . . . . . . . . 39

2.5 Comparing Profit Levels . . . . . . . . . . . . . . . . . . . . . 40

2.6 Comparing Power Production Levels . . . . . . . . . . . . . . . . . . . . 42

2.7 Comparing Water Content across Cases . . . . . . . . . . . . . . . . . . . 43

2.8 Net Cost Analysis under Various Equal Levels of Up-ramping and Downramping Constraints (Coal as Replacement Power) . . . . . . . . . . . . 48

2.9 Net Cost Analysis under Various Equal Levels of Up-ramping and Downramping Constraints (Coal and Natural Gas as Replacement Power) . . . .

3.1 German EEX Spot Price, January 1-July 24, 2006 . . . . . . . . . . . . 53

3.2 German EEX Spot Price, January 9-15, July 17-23, December 18-24, 200658

3.3 Value over Price and Release Rate for the Base and Spike Regimes . . . . . 78

3.4 Optimal Ramping Rate over Price and Release Rate for the Base and Spike Regimes .......................... 79 
3.5 Optimal Ramping Rate over Price and Time for the Base and Spike Regimes 80

3.6 Value over Price and Reservoir Level for the Base and Spike Regimes . . . 85

3.7 Optimal Ramping Rate over Price and Reservoir Level for the Base and Spike Regimes . . . . . . . . . . . . . . . . . . . . 86

3.8 Total Profit vs Ramping Rate Restrictions at Half Release Rate and Full Reservoir Level and at Full Release Rate and Full Reservoir Level . . . . . 87

4.1 Value over Price and Release Rate for the Base, the First Spike, and the Second Spike Regimes (Case I) _ . . . . . . . . . . . . . . . 102

4.2 Optimal Ramping Rate over Price and Release Rate for the Base, the First Spike, and the Second Spike Regimes (Case I) . . . . . . . . . . . 103

4.3 Optimal Ramping Rate over Price and Time for the Base, the First Spike, and the Second Spike Regimes (Case I) . . . . . . . . . . . . . . . 104

4.4 Value over Price and Reservoir Level for the Base, the First Spike, and the Second Spike Regimes (Case I) _ . . . . . . . . . . . . . 105

4.5 Optimal Ramping Rate over Price and Reservoir Level for the Base, the First Spike, and the Second Spike Regimes (Case I) . . . . . . . . 106

4.6 Total Profit vs Ramping Rate Restrictions at Half Release Rate and Full Reservoir Level and at Full Release Rate and Full Reservoir Level (Case I) 108

4.7 Value over Price and Release Rate for the Base, the First Spike, and the Second Spike Regimes (Case II) . . . . . . . . . . . . . . . . . 111

4.8 Optimal Ramping Rate over Price and Release Rate for the Base, the First Spike, and the Second Spike Regimes (Case II) . . . . . . . . . . . .

4.9 Optimal Ramping Rate over Price and Time for the Base, the First Spike, and the Second Spike Regimes (Case II) _ . . . . . . . . . . . 113

4.10 Value over Price and Reservoir Level for the Base, the First Spike, and the Second Spike Regimes (Case II) . . . . . . . . . . . . . . . . . 114 
4.11 Optimal Ramping Rate over Price and Reservoir Level for the Base, the First Spike, and the Second Spike Regimes (Case II) . . . . . . . . . . 115

4.12 Total Profit vs Ramping Rate Restrictions at Half Release Rate and Full Reservoir Level and at Full Release Rate and Full Reservoir Level (Case II) 117 


\section{Chapter 1}

\section{Introduction}

Hydro power is currently favored as a source of clean energy with several desirable features including no carbon emissions, low operating costs, the ability to meet peak demands, significant operational flexibilities and high reliability. In an era of deregulated electricity markets, global climate change and volatile prices for fossil fuels, these key features of hydro power become extremely valuable. However, many studies show that hydroelectric production can also have significant negative effects on the environment including impacts on the aquatic ecosystem due to changes in in-stream flow rates, reservoir levels, and water temperatures which cause changes in the chemical and physical composition of the released water. In addition flow fluctuations can impact beaches and cause bank erosion affecting shore areas that provide critical wildlife habitat for native fishes and other aquatic flora and fauna (Edwards et al. [1999]).

There are numerous studies in the biological and environmental sciences literature exploring these negative consequences of hydro operations. There is a consensus that modified water flows are affecting fish and fish habitat, but the response varies widely. The extent of these negative environmental impacts is case specific, depending on the size of hydro operation and the fragility of the surrounding ecosystem including the characteristics of the water body that receives the released water. Examples of studies documenting these effects include Murchie et al. [2008] and Marty et al. [2009]. Niu and Insley [2013] reference 
numerous other studies which examine the environmental consequences of altered water flows caused by hydro operations. The consequences for the surrounding ecosystem may be judged to be serious enough to warrant restrictions on the management of water flows by a hydro operator. This is an issue that has received attention in numerous jurisdictions across North America. Currently, in Ontario both electricity producers and the Ontario Ministry of Natural Resources are interested in testing whether restricting ramping rates through turbines at hydroelectric facilities can provide ecological benefits without unduly affecting hydro production (Smokorowski et al. [2009]). Some examples of hydro dams that operate with ramping constraints include the Glen Canyon Dams in Arizona (Veselka et al. [1995] and Harpman [1999]); the Sugar Lake Dam in British Columbia (BC Hydro [2005]); and the Kerr Dam in Montana (Flathead Lakers [2005]).

Hydro operators seeking to maximize profits face a complex dynamic optimization problem. The production of electricity depends in a non-linear fashion on the speed of water released through turbines as well as on the reservoir head, which refers to the height of the water in the reservoir. Releasing water at any given hour reduces the head and hence negatively affects the amount of power that can be produced in the next hour. Eventually the released water will be recovered through water inflow into the reservoir. Maximizing profits over time is thus a balancing act between water inflow to and outflow from the reservoir while attempting to match changing electricity demands over time. In additions, hydro power stations are typically required to meet operational, physical and legal requirements. With no restrictions, hydroelectric facilities will maximize profits by adjusting water flows so that electricity production is highest when it is most profitable. This implies hydropower stations will tend to increase the rate of water flow (or ramp up) when prices/demands are high and decrease the rate of water flow (or ramp down) when prices/demands are low to let water levels in the dam recover. Any restrictions imposed on water flows reduce efficiency, profitability, and the ability to react to changes in electricity demand and price.

Ramping rate restrictions are believed to provide environmental benefits by protecting downstream fish, fish habitat and the productive capacity of the river. However, to the extent that hydro production is affected, there will also likely be offsetting impacts on other 
sources of electric power generation such as thermal generation from coal, oil or natural gas. This would have added environmental consequences since thermal power is associated with emissions of greenhouse gases, and pollutants such as $\mathrm{SO}_{2}$ and $\mathrm{NO}_{2}$. Hence in evaluating ramping rate restrictions it is important to study the trade offs between protecting aquatic ecosystems and the optimal operation of hydropower plants to satisfy electricity demand. In much of the existing research on electric power scheduling, ramping rate restrictions are regarded as physical/technical constraints in optimization models, ${ }^{1}$ rather than legal/policy constraints. There are currently only a limited number of studies that estimate the extra costs and associated benefits of restricting ramping at hydroelectric generating stations.

In this thesis, we examine the effect of ramping rate restrictions imposed by a regulatory authority on a power plant's operation and profit. Using a model of a prototype hydro plant we examine the tradeoff between the desire for stricter control of water flows to protect the aquatic environment and the need to vary water flow in response to changing electricity demand and price. We also consider the impact of ramping restrictions on the need for alternate sources of power which may also have undesirable environmental consequences. This thesis will contribute to our understanding of the economic issues involved in imposing ramping restrictions on hydro electric facilities.

In the second chapter, we examine the impact of flow regulations on optimal hydro operations and profitability. We solve a dynamic non-linear model of the profit maximizing decision by a private hydro operator given restrictions on flow rates imposed by a regulator. We assume the hydro plant must meet a minimum level of contract electricity demand and if unable to do so through hydro production, must buy on the spot market. Using data that are representative of a medium-sized hydro operation in Ontario, we investigate the operator's optimal decisions regarding hydro production and power purchases in on-peak and off-peak periods and examine the sensitivity of profits to ramping rate restrictions. We abstract from the issue of electricity price uncertainty by assuming on- and off-peak prices are known and constant.

In principle, the optimal ramping rate restrictions can be identified through a cost

\footnotetext{
${ }^{1}$ Thermal units, especially large and efficient units, frequently have the most significant ramp limits in the system (Svoboda et al. [1997]). In this thesis, we only consider the ramping issue for hydro units.
} 
benefit analysis which includes the lost profit for the hydro operator, the environmental impact of changed reliance on thermal power, and the environmental benefit for the river ecosystem. We assume that any change in hydro production implies an equal offsetting change in thermal electric generation - either coal or natural gas. We estimate the environmental cost or benefit of this change in thermal production due to the resulting change in air pollution emissions. Currently, there are no studies available to measure the environmental benefit of ramping restrictions for the river ecosystem in terms of monetary values. However we undertake to estimate the other two impacts to provide a lower bound for the environmental benefit of ramping rate restrictions that would be required in order for them to be worthwhile. This chapter has identified some of the important trade offs which include the impact on hydro operator profits as well as the environmental impact of a change in the intensity of use of other types of power. However, these results are obtained under relatively restrictive assumptions. For example, the optimization model is solved in a deterministic framework. Uncertain electricity prices as well as water inflows imply that optimal hydro power operation is best studied in a stochastic framework.

In the third chapter, we investigate the impact of ramping restrictions on hydro plant operations and profitability using a Markov regime switching model of electricity prices. Hydro operations are modelled as a stochastic control problem subject to various physical and environmental constraints. Under the risk adjusted measure, we use the standard hedging approach in options pricing to derive the Hamilton Jacobi Bellman Partial Differential Equation (HJB-PDE) for the value of the hydro power station. The optimal ramping rate for water releases is obtained by maximizing the value of the hydro plant. The optimal control is determined by solving the HJB-PDE numerically using a fully implicit finite difference approach with semi-Lagrangian time stepping. Using the same prototype hydro plant as chapter 2, we examine the impact of a range of ramping restrictions on hydro plant operations and profitability. We focus on how the impact of ramping restrictions depends on the characteristics of the assumed price process such as the volatility, probability of regime shift, speed of mean reversion, and long run average price and relies on the cost of generating hydroelectric power (relative to the price level). For comparison we also examine results for a single price regime case to determine how the presence of two price 
regimes affects hydro plant value and optimal operations. Given the problematic nature of estimating the environmental benefits or costs of different ramping rate restrictions, we seek instead to examine the other side of the equation - the costs of these restrictions in terms of lost profitability. Knowledge of these costs will help illuminate the trade offs involved and inform the design of regulations.

In the fourth chapter, we make a further investigation on the impact of ramping restrictions by extending the Markov regime switching model in chapter 3 to a model with multiple jump sizes. In the empirical analysis, we conduct numerical experiments on the jump sizes by proposing a regime switching model with three regimes: the base regime, the first spike regime, and the second spike regime. Assuming different jump sizes among these three regimes allows us to study the effect of multiple jump sizes on the impact of ramping restrictions on the hydro plant. The different jump sizes among these three regimes could be viewed as the simplest approximation of the random jump size observed from the data.

The main contribution of the thesis is in providing a comprehensive analysis of the economics of ramping rate restrictions of hydro power plants for the purpose of environmental protection. In both deterministic and stochastic settings, the thesis provides insights into our understanding of ramping related issues for a hydro power station, including the optimal choice of ramping restrictions and possible policy recommendations. This thesis extents the existing literature as follows:

- The empirical studies in this thesis are based on a unique data set for a prototype hydro power plant in Ontario.

- Chapter 2's contribution to the existing literature is in furthering our understanding of the trade offs involved when ramping rate restrictions are imposed at hydro facilities. In particular, we examine the sensitivity of hydro station profits to ramping restrictions as well as the potential impact on hydro electricity production and production from alternative generating facilities.

- Chapter 2 contributes to the literature by using a replacement power approach (assuming that any change in hydro production implies an equal offsetting change in 
thermal electric generation) to measure the net cost of ramping rate restrictions (as the loss in profit from hydro generation net of the value of any implied change in polluting emissions from thermal plants). This net cost for the prototype hydro plant may be viewed as the lower bound needed for the value of aquatic ecosystem benefits of ramping restrictions for these restrictions to be worthwhile.

- Chapter 3 contributes to the literature by studying the impact of ramping restrictions on hydro plant operations and profitability using a regime switching model of electricity prices, which we argue provides a more realistic characterization of electricity prices than other models, such as jump diffusion, used previously in the literature.

- Chapter 3's contribution to the literature is in furthering our understanding of how the impact of ramping restrictions depends on the cost of generating hydroelectric power and the characteristics of the assumed price process such as the volatility, probability of regime shift, speed of mean reversion and long run average price, and how the presence of two price regimes affects hydro plant value and optimal operations and the impact of ramping restrictions on hydro plant.

- Chapter 4 contributes to the literature by empirically investigating the impact of ramping rate restrictions on hydro plant operations and profitability using a regime switching model with multiple jump sizes.

The main results of the thesis can be summarized as follows:

- In chapters 2,3 , and 4, for our medium-sized power plant we observe profits are significantly affected for the most restrictive ramping constraints, but we also observe a range of less severe ramping restrictions over which profits are not substantially affected. This suggests that for some levels of ramping restrictions, the aquatic environment can be properly protected while allowing hydro dams to maintain profitable operations.

- In the second chapter, we find that ramping restrictions negatively affect profits to the extent that they force a hydro operator to make different choices (on hydro production 
and power purchases) than when no ramping restrictions are imposed. One counter intuitive result is that ramping restrictions can cause an increase in the total amount of hydro power produced over a 24 hour period. Assuming that any change in hydro production implies an equal offsetting change in thermal electric generation, the increased hydro production constitutes an added environmental benefit of ramping restrictions from reduced air pollution emissions, in addition to any benefits to the aquatic ecosystem below the hydro dam.

- In the third chapter, we observe that in most scenarios the optimal control is of a "bang-bang" type (ramping up or down at the upper limit). The exception to this is when the dam is up against one of the other constraints such as maximum/minimum release rates or maximum water content in the dam. We find that ramping restrictions have a larger effect in an environment where frequent ramping up or down is desired. A lower speed of mean reversion, higher volatility and more frequent transition to the spike regime all have the effect of increasing the impact of ramping restrictions. The results also show that price spikes have a significant effect on the value of the hydro power plant, however the ramping effect on profits mostly depends on the level of the price relative to the cost of generation (how long and how frequent the price is close to or below the cost), but is not very sensitive to the price jumps.

- In the fourth chapter, our numerical experiments provide further evidence that ramping restrictions have a larger impact when the expected variation in price is increased such as through an increase in jump size (for the third regime) which makes it desirable to change water release rates relatively frequently.

Results from this research should facilitate the implementation of environmental regulations designed to promote the integrity of river systems, as well as to provide a set of planning tools regulators and industry can use to negotiate the optimal ramping rate for environmental and economic benefits. 


\section{Chapter 2}

\section{On the Economics of Ramping Rate Restrictions at Hydro Power Plants: Balancing Profitability and Environmental Costs}

\section{$2.1 \quad$ Introduction}

In this chapter, we examine the effect of ramping rate restrictions imposed by a regulatory authority on a power plant's operation and profit. We model profit maximization of a prototype hydro plant, based on a medium size plant in Ontario. Assuming that the plant must satisfy a minimum contract demand either through producing hydro power or purchasing power on the spot market, we investigate the operator's optimal decisions regarding hydro production and power purchases in on-peak and off-peak periods. We abstract from the issue of electricity price uncertainty by assuming on- and off-peak prices are known and constant $^{1}$.

\footnotetext{
${ }^{1}$ In this chapter, the profit maximization model is kept fully deterministic because it allows us to include extra control variables and restrictions to implement the replacement power approach. This determinis-
} 
We also consider the potential impact of ramping restrictions on the need for other sources of power generation. We assume that any change in hydro production implies an equal offsetting change in thermal electric generation - either coal or natural gas. We estimate the environmental cost or benefit of this change in thermal production due to the resulting change in air pollution emissions.

There are currently no suitable monetary measures available in the literature of the environmental benefit for the river ecosystem of ramping restrictions. In the absence of such monetary measures, we determine the net cost of ramping restrictions as the loss in profit from hydro generation net of the value of any implied change in polluting emissions from thermal plants. This net cost for the prototype hydro plant may be viewed as the lower bound needed for the value of aquatic ecosystem benefits of ramping restrictions for these restrictions to be worthwhile.

This chapter's contribution to the literature is in furthering our understanding of the trade offs involved when ramping rate restrictions are imposed at hydro facilities. In particular, we examine the sensitivity of hydro station profits to ramping restrictions as well as the potential impact on electricity production from hydro and other sources. Although the impact of ramping restrictions on firm profits will depend on the specifics of the particular hydro plant under consideration, as well as the market structure that the plant operates in, we are able to draw some general conclusions. Ramping restrictions will have a negative effect on profits to the extent that they force a hydro operator to make different choices than when no ramping restrictions are imposed. The most obvious choice variable affected is the allocation of power sales over a given day. Profits are reduced if ramping restrictions force hydro operators to increase the amount of power sold in off-peak periods when prices are lower. In our analysis we observe a significant effect on profits for the most restrictive ramping constraints, but we also observe a range of ramping restrictions over which profits are not substantially affected.

A more surprising result is that ramping restrictions can cause an increase in the total

tic model is clearly limited since it ignores the stochastic nature of electricity prices and water inflows. However, in a dynamic optimization model with stochastic electricity prices and water inflows, it would be notoriously difficult to conduct the replacement power analysis. 
amount of hydro power produced over a 24 hour period. This is a consequence of hydro operators' efforts to maintain profits in the face of constraints. In response to the ramping constraints, operators increase power production in off-peak periods while at the same time attempting to maintain production as much as possible in on-peak periods. If the increased hydro production implies a reduction in power produced by fossil fuel fired plants, this may constitute an added environmental benefit of ramping restrictions, in addition to any benefits to the aquatic ecosystem below the hydro dam.

It is important to note, however, that the analysis in this chapter is for a single hydro plant. If ramping rate restrictions were applied to a significant portion of the hydro generation capacity in the a particular province or state, then the impact on the entire grid would need to be considered. In this chapter we assume that even though ramping restrictions constrain the system's ability to meet peak demand with hydro, it is possible to meet those peak demands with other electricity sources at little increase in cost. A full analysis of ramping restrictions on a significant portion of hydro generation would need to consider the potential for increased cost in meeting peak demands by operating thermal units less efficiently, or by adding more expensive gas-fired units.

This chapter is organized as follows: in the second section, we provide a brief review of the related literature; assumptions and model formulation are presented in section three; then we formally specify the optimization problem; data issues are addressed in section five; next we calibrate the power generation function and the head function ${ }^{2}$; section seven contains the empirical analysis of the hydro plant operation and profit; the environmental impact of changes in thermal generations is considered in section eight; lastly, conclusions and directions of future research are given in section nine.

\section{$2.2 \quad$ Literature Review}

The literature on hydro dam operations and the associated environmental effects is enormous. The existing research in this area can be divided into three broad categories: the

\footnotetext{
${ }^{2}$ The head refers to the difference in height between a dam's water source and water outflow.
} 
power and civil engineering literature; the biology and environmental studies literature; the energy and environmental economics literature. In this section we survey a selection of papers from each of these literatures with an emphasis on ramping related issues.

In the power engineering literature, there has been considerable interest in the application of mathematical programming methods to scheduling the generation of electricity. Most of this work has focused on problems of scheduling the generation of hydro-electricity or thermal electricity, and coordinating thermal electricity generation with hydro-electricity generation. Much of the interest in electricity scheduling models concentrates on the optimal operation of power stations with the objective of producing electric power at the lowest cost, at maximum profit, at the best efficiency, at maximum potential energy and so on. In general, these models include many detailed technical specifications and constraints are quite complex. Their solution is computationally intensive, requiring special solution algorithms. ${ }^{3}$ From the power engineering literature, some of the papers studying the optimal production scheduling problem for a hydro-electric power producer include: Hreinsson [1988], Soliman and Christensen [1988], Shawwash et al. [2000], Conejo et al. [2002] and Deng et al. [2006].

In practice, hydro operators face regulatory requirements for minimum and maximum water flows and levels, as well as restrictions on ramping rates which are intended to protect the aquatic environment of the associated rivers and lakes. Some of the studies discussed above include only minimum flow restrictions in their optimization models as physical or environmental constraints. A few of them consider ramping rate restrictions as physical constraints. In the international literature on power engineering, including the papers mentioned above, relatively little attention has been paid to directly address ramping rate restrictions as policy constraints for environmental protection. One exception is Guan et al. [1999], where an optimization-based algorithm is presented for scheduling hydro power systems with restricted operating zones and discharge ramping constraints. In the Guan paper the ramping constraints may be imposed on discharges for generation or spillage through canals or tunnels due to the requirements of navigation, the environment, recreation, etc. They find that, with ramping constraints imposed, the hydro production

\footnotetext{
${ }^{3}$ See Bensalem et al. [2007] for example.
} 
schedule changes significantly and the costs are generally increased, since the constraints limit the water release so that downstream power plants may generate less power.

The term 'hydropeaking' is used in the literature to refer to the shifting of hydro production to periods in the day when prices are highest. The environmental effects of hydropeaking power generation on fish and fish habitat have attracted much attention from biological and environmental scientists. Most of their studies directly address the effect of the instream flow rate (the minimum flow rate, the variation of flow rate and the ramping rates) of regulated rivers on the downstream biological habitat. In Scruton et al. [2003], hydropeaking or pulse power generation is defined as "reservoir operations, where water is stored to generate electricity during times of peak demand, leading to diurnally and annually variable water pulses in the river below the power station resulting in unnatural flow patterns involving alterations to magnitude, duration, sequence, and frequency of flows." They note that hydropeaking often results in "rapid changes in river discharge and associated habitat conditions over very short time scales (less than a day, or multiple peaks per day) and changes can be moderate or as large as several orders of magnitude." There is a clear consensus that modified flow regimes in regulated rivers mainly for purposes of hydroelectric generation are affecting fish and fish habitat, but the severity and direction of the response varies widely. Murchie et al. [2008] conduct a systematic review of available literature examining the response of fish to fluctuating flow regimes in different systems.

In regulated rivers, the environmental heterogeneity of fish habitat may be aggravated and unpredictable, depending on hydropower demand and price. One consequence experienced in many rivers is peaking flow on a daily basis, with suddenly increasing and high flows in the morning and increasingly higher flows during the day, then decreasing flow in the evening, and extremely low flow at night. Hvidsten [1985], Cross and DosSantos [1988], Bradford et al. [1995] and Saltveit et al. [2001] demonstrate that this variable flow pattern affects the habitat conditions and directly results in stranding of young fish and increased mortality. The negative impacts of hydropeaking are also documented by Flodmark et al. [2002], Berland et al. [2004], Scruton et al. [2003], Scruton et al. [2005], Scruton et al. [2008] and Grand et al. [2006]. Freeman et al. [2001] demonstrate that providing periods of stable flow conditions below hydropower facilities during appropriate seasons should 
facilitate reproduction by native riverine fishes. Marty et al. [2009] find that there is a significant effect of a high ramping rate flow regime on the length of the food web. The operations of waterpower facilities will alter a river's flow in terms of its magnitude, timing, frequency, rate of change, and duration. A publication of the Ontario Ministry of Natural Resources [2003] summarizes some negative effects of this alteration, both from up-ramping and down-ramping. Specifically this report states that excessive up-ramping could affect fish holding instream and result in scouring of substrate and infauna (Cushman [1985]), while slower down-ramping is beneficial for biota by protecting fauna from stranding and ensuring better conditions for vegetation seeding (Petts and Maddock [1994]).

Relationships between the quantity of suitable fish habitat and flow have been used to select regulatory minimum flows for numerous rivers (Jager and Smith [2008]). Currently, there is considerable interest in Ontario in using and evaluating instream-flow-needs (IFN) methods for fish. Kilgour et al. [2005] provide a review of IFN methods appropriate to waterpower facilities. Gouraud et al. [2008] estimate the change in brown trout population under different minimum instream flows. Murchie et al. [2008] suggest that more studies are needed to evaluate the behaviour of fish during dynamic periods such as flow increase or decrease (i.e. during the ramping). Jager and Smith [2008] review research on reservoir optimization problems that explicitly includes environmental objectives. They find that nearly half of the studies they reviewed addressed environmental flows by including a constraint on minimum flow releases.

From our survey of the available information it appears that many hydro dams operate with minimum flow requirements, but very few operate under ramping rate constraints. Some examples that do face ramping constraints include the Glen Canyon Dams, located on the Colorado River in Arizona, which are operated under restrictions on maximum flows, minimum flows, ramp rates, and the daily change in flow (Veselka et al. [1995] and Harpman [1999]). Located on the Shuswap River, east of Vernon in the southern interior of British Columbia, the Sugar Lake Dam is operated under ramp rate constraints and the Wilsey Dam needs to meet the minimum discharge requirement (BC Hydro [2005]). The Kerr Dam on the Flathead River about five miles southwest of Polson in Montana faces the following restrictions: minimum flow requirements, maximum between-day flow 
changes and maximum allowable ramping rates (Flathead Lakers [2005]).

In the economics literature, there are very few studies regarding the environmental effect of ramping and the associated economic impact on hydro power operations. Some of these studies include Veselka et al. [1995], Edwards et al. [1999], Edwards [2003], Harpman [1999] and Chen and Forsyth [2008], who treat ramping rate restrictions as environmental constraints in their optimization models. These papers (except Chen and Forsyth [2008]) assume that the power stations operate under a particular ramping rate regime, but do not analyze the effect of various levels of ramping rate restrictions on the power station's optimal operation and profit. The trade offs involved in the choice of the optimal ramping rate regime are not addressed. We attempt to fill in this gap in the literature by considering both the associated benefits and costs of ramping restrictions on hydro profits and on total daily hydro production and the potential implications for other sources of power.

There are some related cost and benefit studies similar to this chapter. Kotchen et al. [2006] conduct a benefit-cost analysis of changing daily conditions from peaking to run-ofriver (ROR) flows for two hydroelectric dams in Michigan. They consider three categories of costs and benefits related to the switch to ROR flow: electricity production costs, air quality benefits, and recreational fishing benefits. Huppert [1999] estimates the costs of protecting the endangered and threatened salmon, including the cost of environmental restrictions on hydropower operations in the Snake River. Jager and Bevelhimer [2007] review hydropower projects with license-mandated changes from peaking to ROR operation, and discuss producer costs and environmental benefits associated with operations: decreased generation efficiency; higher energy cost of fossil fuels needed to replace hydropower during peak versus off-peak hours; the negative costs of environmental externalities.

\subsection{Assumptions and Model Formulation}

The goal of the chapter is to examine the opportunity cost of ramping rate restrictions on hydro power operations. Our approach is to consider the costs for a representative hydro power plant. We assume that the hydro plant has signed a binding contract to supply a 
specified amount of power to a certain customer, which implies there is a minimum amount of power that the plant must produce. Any power which is over and above the contracted amount can be sold in the spot market. This is similar to the assumption in Veselka et al. [1995], but contrasts with Edwards et al. [1999] in which the contract demand must be met exactly with no production allowed over the contracted amount.

This hypothetical contract is a device to permit the estimation of the cost of ramping restrictions. If ramping restrictions imply that the contract cannot be met at certain times during the day, then another source of power must be purchased. The additional cost of this alternative source of power is a measure of the opportunity cost of the ramping restrictions.

The contract specifies the quantity of electricity exchanged for each time $t$. The price is the spot market rate which is assumed known and non-stochastic. This is clearly unrealistic, since a key feature of electricity prices is their high degree of volatility. In this chapter we ignore uncertainty in both electricity demand and price, and focus solely on the opportunity costs of ramping restrictions in a non-stochastic environment. The case of uncertain demand and price is left for future research.

We also ignore the possibility that the hydro power station could provide any ancillary services to the electricity market. For example, besides producing electricity, a hydro unit can also provide spinning reserves, which means that some of its power capacity is put aside to provide electricity in case of a power shortage somewhere over the network.

A typical hydroelectric generation system can consist of more than one independent rivers, with one or several generating facilities and reservoirs in a series or in parallel, and transmission lines to neighboring systems through which electricity may be exchanged. In addition, reservoir management deals not only with power generation, but with recreation, fishery and irrigation as well. In order to focus on the ramping issue of the station's operation, the proposed model will only consider the power generation aspect of one representative station and issues related to system transmission and distribution are ignored in this study. In brief, we will largely follow Edwards et al. [1999] in the theoretical formulation of the model. Specific differences are noted in the model description later in this 
section.

The time horizon of the model is $T$ periods, with each individual period indexed by $t=1, \ldots, T$. In the empirical example to follow we solve the optimization problem for each hour over a five day period. In this case $t$ represents one hour and $T=5 X 24=120$ hours. We denote the number of days as $N$ where $N=5$. Each day is further divided into on-peak and off-peak periods. We assume that the prevailing spot price during peak periods will exceed that for off-peak periods. For the prototype hydro power station, there are three alternative choices available to meet the contract demand: generation of hydro power only; purchase of electricity on the spot market at prevailing prices and resale to the consumer; or some combination of these two. The hydro power station operates under various physical constraints and must also meet environmental and other policy constraints set by the regulator. Here, we assume that the hydro operation is subject to the following constraints:

- Maximum hourly up-ramping and down-ramping rates;

- Maximum daily total water release. ${ }^{4}$

- Maximum and minimum hourly

- water release rates;

- water spill rates;

- head requirements;

- water content;

- hydro power generation;

In addition, contract demand and the water balance equation must be satisfied at all times.

\footnotetext{
${ }^{4}$ In this model we do not explicitly include a constraint on the maximum daily change in water flow. Normally, up-ramping and down-ramping are believed to have more a severe effect on the environment compared with fluctuations in daily flow. Since our main focus is on the ramping rate, we assume the allowable fluctuations in daily flow are large enough that our optimization results are not affected.
} 
The total amount of power provided to the market by the owner of the hydro station comprises the portion derived from hydro generation and the portion derived from spot market purchases and resale. For a specific hour, this can be written as follows:

$$
q_{t}=q_{t}^{r}+q_{t}^{h}\left(r_{t}, h_{t}\left(w_{t}\right)\right)
$$

where $q_{t}$ is the total electricity supplied by the owner during period $t ; q_{t}^{r}$ is the electricity purchased from the spot market for resale; and $q_{t}^{h}$ is the amount of hydro power generated and sold in period $t$. Hydro generation is a function of $r_{t}$, the water release rate during period $t$, and $h_{t}$, the head of the dam which depends on the amount of water in the reservoir $w_{t}$. The hydro power production function will be non-linear and is assumed to be continuous and increasing with respect to both arguments ${ }^{5}, \frac{\partial q_{t}^{h}}{\partial r_{t}}>0$ and $\frac{\partial q_{t}^{h}}{\partial h_{t}}>0$, but the second order derivatives are assumed to be zero, i.e., $\frac{\partial^{2} q_{t}^{h}}{\partial^{2} r_{t}}=0$ and $\frac{\partial^{2} q_{t}^{h}}{\partial^{2} h_{t}}=0$. We further assume that $q_{t}^{h}\left(0, h_{t}\left(w_{t}\right)\right)=0$ and $q_{t}^{h}\left(r_{t}, 0\right)=0$, meaning that at any level of water head when there is no water release the hydro power generation will be zero, and at any level of water release rate, if the water head is zero there will be no power generated. For the head function $h_{t}\left(w_{t}\right)$, it is assumed that $\frac{\partial h_{t}}{\partial w_{t}}>0, \frac{\partial^{2} h_{t}}{\partial^{2} w_{t}}=0$ and $h_{t}(0)=0$. The specific functional form assumed for the hydro production function is given in Section 2.6.

Next, we assume that the equation of motion for water is governed by the following formula ${ }^{6}$ :

$$
w_{t+1}=w_{t}+\alpha\left[i_{t}-r_{t}-f_{t}\right]
$$

This equation states that the total amount of water in the reservoir at time $t+1$, i.e., $w_{t+1}$, equals to the total amount of water stored at time $t$, i.e., $w_{t}$, plus the water inflows (coming from snow melting, rain, runoff water and natural river flow) into the reservoir at time $t$, i.e., $i_{t}$, minus the water outflows (turbine and spill flows) at time $t$, i.e., $\left\{r_{t}, f_{t}\right\}$. $\alpha$ is

\footnotetext{
${ }^{5}$ The more realistic hydro power production function is not continuous, but our simplified assumptions still allow a good approximation of the actual function (see Harpman [1999]). In the empirical studies, we use the production function given by Equation (2.26). It should be pointed out that for any specific dam, to apply this production function, both the water release rate and water head should be within certain limits as described in Equations (2.6) and (2.8)-(2.9). Normally, the upper and lower limits will be different for various dams.

${ }^{6}$ This water balance equation differs from the one in Edwards et al. [1999] by the inclusion of spill flows.
} 
the conversion factor to convert water flow units into water volume units. In this chapter, water flows are measured in Cubic-feet-per-second (CFS) and water volume is in acre feet. Reservoir water losses due to seepage and evaporation are neglected ${ }^{7}$. Here, the dam possesses a mechanism to release water with and without hydro power generation. This general formulation captures the case when it may be necessary to spill a large quantity of water such as during a period of flooding. In practice the spill flow can be controlled quite precisely by adjusting gate openings. However, spilling should be avoided as much as possible, given that no electricity is produced in this case.

In addition, the hydro power station is required to meet contractual obligations for power at any time of the day, so the sum of hydropower production and the purchased power for resale must be sufficient to satisfy the contract demand of the day. This load resource balance can be represented by the following equation:

$$
\hat{q}_{t} \leq q_{t}^{r}+q_{t}^{h}\left(r_{t}, h_{t}\left(w_{t}\right)\right)
$$

$\hat{q}_{t}$ represents the contract demand during period $t$ of the day.

We assume that the hydro power station is subject to the up-ramping and downramping constraints which will limit its operational ability to increase or decrease the water release rate in any given period. These two constraints can be expressed as:

$$
\begin{aligned}
& r_{t+1}-r_{t} \leq r^{u} . \\
& r_{t}-r_{t+1} \leq r^{d} .
\end{aligned}
$$

$r_{t}$ refers to water release in period $t$, which may be an on- or off-peak period. Equation (2.4) limits the rate at which the water release rate can be increased between periods to $r^{u} .8$ The up-ramping limit will be determined by the physical capabilities of the particular hydro turbine and the ramping rate constraint imposed by regulators to protect the environment. In this chapter we concern ourselves only with the latter source of ramping restrictions.

\footnotetext{
${ }^{7}$ These assumptions are reasonable for a very short term analysis such as five days in this chapter.

${ }^{8}$ In general the desired ramping restrictions my vary over the hours in a day, and over months and seasons as well. In this chapter we assume fixed ramping constraints.
} 
Similarly, Equation (2.5) limits the rate of ramping-down, i.e., the rate at which the water release rate can be decreased between periods. Again we assume the ramping constraint is imposed by regulators, although the physical characteristics of a particular hydro unit may also limit down-ramping.

The hydro station also faces minimum and maximum water release rate requirements, which can be represented as:

$$
r^{\min } \leq r_{t} \leq r^{\max }
$$

Equation (2.6) limits the range of water release rate by $r^{\text {min }}$ and $r^{\text {max }}$. Again we assume the minimum and maximum water release rates are constant over any day and represent regulatory requirements to protect the river ecosystem. The minimum release requirement is loosely defined as the smallest amount of flow that can be left in the river without harming downstream fish populations (Jager and Smith [2008]). By imposing these constraints, the hydro power station's operational flexibility may be significantly affected. Currently, many hydro power stations operate under the minimum and maximum water release constraints.

Similarly, the station faces minimum and maximum water spill rate requirements (Catalão et al. [2006]), denoted $f^{\min }$ and $f^{\max }$ respectively. These can be represented as:

$$
f^{\min } \leq f_{t} \leq f^{\max }
$$

In practice, especially during flood periods, spillways may release water so that the water does not overtop and damage the dam. Spillways provide added flexibility of operations given variations in water inflow.

Additional operational constraints include that the water level must remain between specified minimum and maximum values. This implies the station faces minimum and maximum water head requirements (Equation (2.8)), and upper and lower reservoir storage constraints (Equation (2.9)), which may vary over the year (Catalão et al. [2006]). These constraints can be stated as:

$$
\begin{gathered}
h^{\text {min }} \leq h_{t} \leq h^{\text {max }} \\
w^{\text {min }} \leq w_{t} \leq w^{\text {max }} .
\end{gathered}
$$


where, the water head lower bound is $h^{\text {min }}$ and the water head upper bound is $h^{\text {max }}$. The reservoir storage lower bound is $w^{\text {min }}$ and the reservoir storage upper bound is $w^{\text {max }}$.

We further assume that the hydro station is facing minimum and maximum power production constraints, which can be written as:

$$
q^{\min } \leq q_{t}^{h} \leq q^{\max }
$$

These limits may be technical limits of hydro turbines or may reflect a constraint on the amount of power that can be transmitted through power lines, perhaps due to congestion.

According to Edwards et al. [1999] and Harpman [1999] hydro dams typically are required to release a specified quantity of water each month. For example, in the United States Power Marketing Administrations (PMAs) are required to release specific amounts of water for each dam during each month of the year (Edwards et al. [1999]). In this chapter the optimization occurs over 5 days and it is assumed that there is a maximum that can be released in each 24 hour period. The constraint is given by:

$$
\sum_{t=1}^{24} \alpha r_{t j} \leq R, j=1, \ldots, N
$$

where $\alpha$ is the conversion factor to convert a water flow into a water volume and $j$ indexes each day. Additional optional constraints in the model can be easily imposed if required, such as system reliability and ancillary service requirements; more detailed market conditions; transmission losses and other operational details.

\subsection{The Optimization Problem}

In this section we formulate the optimization problem for the representative hydro power station. The owner of the station is assumed to maximize profits subject to various constraints described in the previous section. Profit maximization involves determining the amount of power production which depends in a non-linear fashion on water released 
through the turbine and on dam head, as given in Equation (2.1). Dam head is a reflection of water content in the reservoir. A hydro operator knows that water released today reduces dam head and therefore the amount of power that can be produced in the next period. Profit maximization over time involves choosing the level of water releases so that the benefit in terms of electricity production today just offsets the opportunity cost in terms of foregone future production and profits.

In order to keep our optimization problem of manageable size, our empirical analysis considers a 5 day period ${ }^{9}$ of operations. The optimal choices in any single day depend on initial conditions, and in particular on the initial water content and dam head. To avoid dependence on arbitrary initial conditions, we look for a steady state solution where the optimal choice of water release and water level in the dam is unchanging from the previous day. In our empirical example, we choose initial conditions for water level and the water release rate that allow us to reach a steady state within the five day period. We then report the results for a steady state day in all cases.

Our focus in this chapter is on ramping rate constraints, Equations (2.4) and (2.5), and we measure their cost as the lost profit from having to meet these constraints, net of the cost of any change in pollutant emissions caused by a change in the economy's reliance on thermal power. As noted earlier, we do not attempt to specify the benefits of environmental restrictions in terms of reduced damages to the aquatic environment. This is beyond the scope of the current chapter.

The representative hydro station's power generation is assumed to be small in the electricity market and hence is a price-taker during each period. The station charges the spot market price for its power, whether generated by the station, purchased from the spot market for resale, or some combination of the two. The power purchased for resale is purchased and sold at the same spot price, so no net revenue is generated. However it is assumed that an administrative cost is incurred, $c_{t}^{r}$ per $\mathrm{kWh}$, so the hydro station incurs a net loss on this transaction. A similar assumption is made in Edwards et al. [1999].

\footnotetext{
${ }^{9}$ This time period is sufficient for the results to reach (or nearly reach) a steady state. In the empirical studies, the computational time for each case varies from less than an hour to several hours.
} 
This administrative cost ${ }^{10}$ can be an arbitrarily small number, but is required to achieve a reasonable solution to the optimization problem in that spot market purchases are only made when needed to meet contract demand. The hydro generation and transmission (G\&T) costs are given by $c^{h}$ per unit of power and are assumed to be the same during both off-peak and on-peak periods. The spot electricity price is denoted by $p_{t}$ per $\mathrm{kWh}$ during time $t$.

The minimum amount of power produced or purchased by the hydro plant owner is specified in the contract. Therefore, whatever the realized market conditions and water inflows, contract demand must always be satisfied, and purchase for resale may become necessary at some points in time. The option to purchase power in the spot market is valuable to the hydro operator, since it means the contract demand can always be met. In the empirical examples that follow we assume water inflow is deterministic, but in practice the uncertainty and variability of water inflow due to weather conditions may impact the amount of electricity that a hydro station can generate in any given period. The stochastic nature of water flows would give added value to the ability to satisfy contract demand with spot market purchases. In addition, this option also creates value by giving the operator the flexibility of hydro-shifting. Hydro-shifting refers to the practice of shifting production to on-peak periods when prices are highest. In off-peak periods, contract demand can be satisfied through spot market purchases.

The total profit of providing power over the $T$ periods is given by the following equation:

$$
\sum_{t=1}^{T}\left\{\left(p_{t}-c^{h}\right) q_{t}^{h}\left(r_{t}, h_{t}\left(w_{t}\right)\right)-c_{t}^{r} q_{t}^{r}\right\} .
$$

The first term inside the brace accounts for the total profit from generating hydroelectric power, given the hourly spot prices. The second term inside the brace represents the net cost of purchasing power for resale from the spot market. It is the per unit administrative $\operatorname{cost} c_{t}^{r}$ multiplied by the quantity of spot market purchases.

The optimization problem is to maximize Equation (2.12) subject to a suite of con-

\footnotetext{
${ }^{10}$ Assuming a small administrative cost could avoid spot market purchases when not needed.
} 
straints. The set of control variables includes the water release rate for power generation, the water spill rate and the amount of power to purchase for resale for each period $t$, i.e., $\left\{r_{t}, f_{t}, q_{t}^{r}\right\}$. The state variables is the water content, $w_{t}$. Exogenous variables including the water inflow rate, electricity demand, and electricity price for each period $t$, i.e., $\left\{i_{t}, \hat{q}_{t}, p_{t}\right\}$ are assumed to be known and deterministic. This is a deterministic dynamic non-linear optimization problem. The objective function and constraints are given below in Equations (2.13)-(2.24). The constraints ${ }^{11}$ are as detailed in Section 2.3, with the addition of the non-negativity constraint, Equation (2.23).

$$
\max _{r_{t}, f_{t}, q_{t}^{r}} \sum_{t=1}^{T}\left\{\left(p_{t}-c^{h}\right) q_{t}^{h}\left(r_{t}, h_{t}\left(w_{t}\right)\right)-c_{t}^{r} q_{t}^{r}\right\} .
$$

Subject to

$$
\begin{gathered}
w_{t}=w_{t-1}-\alpha\left(r_{t-1}+f_{t-1}\right)+\alpha i_{t-1}, t=2, \ldots, T . \\
\hat{q}_{t} \leq q_{t}^{r}+q_{t}^{h}\left(r_{t}, h_{t}\left(w_{t}\right)\right), t=1,2, \ldots, T . \\
r_{t}-r_{t-1} \leq r^{u}, t=2, \ldots, T . \\
r_{t-1}-r_{t} \leq r^{d}, t=2, \ldots, T . \\
r^{\text {min }} \leq r_{t} \leq r^{\max }, t=1,2, \ldots, T . \\
f^{\text {min }} \leq f_{t} \leq f^{\max }, t=1,2, \ldots, T . \\
h^{\text {min }} \leq h_{t} \leq h^{\max }, t=1,2, \ldots, T . \\
w^{\text {min }} \leq w_{t} \leq w^{\max }, t=1,2, \ldots, T . \\
q^{\text {min }} \leq q_{t}^{h} \leq q^{\max }, t=1,2, \ldots, T . \\
0 \leq q_{t}^{r}, t=1,2, \ldots, T .
\end{gathered}
$$

\footnotetext{
${ }^{11}$ Equations (2.14)-(2.22) and (2.24) represent the equation of motion for water, load resource balance, maximum up-ramping constraint, maximum down-ramping constraint, minimum and maximum water release rate requirements, minimum and maximum water spill rate requirements, minimum and maximum water head requirements, upper and lower reservoir storage constraints, minimum and maximum power production constraints, and maximum daily release constraint respectively.
} 


$$
\sum_{t=1}^{24} \alpha r_{t j} \leq R, j=1, \ldots, N
$$

The Karush-Kuhn-Tucker conditions for this mathematical programming problem can be easily derived. This type of analysis admits two possible solution forms. The first is an interior solution characterized by all endogenous variables having positive values at the optimum (i.e., the dispatcher relies on both thermal power resales and hydro generation in both periods). The second is a corner solution, in which at least one of the endogenous variables will take on a zero value at the optimum (e.g., no thermal power is sold or no hydro power is generated in one of the periods). For the empirical studies in the following sections, we will specify the optimization problem, and obtain solutions using Matlab. ${ }^{12}$

\subsection{Data Description}

The prototype hydro plant used in our empirical example is based on a medium-sized plant in Ontario. We construct our example using some specifications of an Ontario Power Generation (OPG) generating station, as well as our own assumptions based on input from a variety of sources. An example of a medium-sized hydro plant is OPG's Abitibi Canyon generating station located on the Abitibi River in northeastern Ontario. Details of the generating station can be found on the OPG web site, ${ }^{13}$ and in Statistics Canada [2000] and Hendry and Chang [2001]. ${ }^{14}$

OPG owns 65 hydro generating stations with a total capacity of 6,963 megawatts (MW). The Abitibi Canyon station consists of five generating units and has a total generation capacity of about $336 \mathrm{MW}$. In terms of water inflow, the combined physical capacity of the generators is assumed to be about 19 thousand Cubic-feet-per-second (CFS) of water. The

\footnotetext{
${ }^{12}$ Using Matlab's optimization tool fmincon.

${ }^{13}$ For example, see http://www.opg.com/power/hydro/northeast_plant_group/abitibi.asp and http://www.opg.com/power/.

${ }^{14}$ Hendry and Chang [2001] investigated the composition and structure of fish communities, and habitat features in the Abitibi Canyon generating station tailwater. Further information about the Abitibi Station is available in their study.
} 
storage capacity of the reservoir is assumed to be about 17 thousand acre-feet of water.

We model the optimal operation of the hydro station over a 24 hour period assuming it faces a contract demand requirement that ranges from $112 \mathrm{MW}$ during the off-peak period to 336 MW during the on-peak period (Table 2.1). This contract is hypothetical and mimics the daily pattern of Ontario electricity demand. The contract demand can be met either by generating hydroelectricity, or purchasing power from the spot market for resale, or some combination of both.

Consistent with the empirical observations, peak hours are specified as being from 6 AM to 11 PM Mondays through Fridays, and off-peak hours are from 11 PM to 6 AM. Each 24 hour period begins at 11 PM, with 11 PM - 12 PM labeled as the first hour. This can be seen in Table 2.1.

Data for the Hourly Ontario Electricity Price (HOEP) from 01 May 2002 to 30 November 2006 is used to determine reasonable assumptions for electricity prices. Based on the definitions of off-peak and on-peak periods, we calculate the average prices for both periods using these data. The average spot electricity price is $36.33 \$ / \mathrm{MWh}$ during the off-peak period and $62.13 \$ /$ MWh during the on-peak period, which will be used in our empirical analysis (Table 2.1). We also assume that purchase for resale incurs an administrative cost of $2 \$ / M W h$, which will be paid by the hydro operator. This is the amount assumed in Edwards et al. [1999]. In addition, the cost of generating hydroelectric power is assumed constant at $20 \$ /$ MWh in both off-peak and on-peak periods for the hydro station.

Actual water inflows are stochastic in nature, but are handled here in a deterministic manner in this analysis. Based on data for the historical water inflow for the Abitibi Canyon from 01 January 2001 to 30 November 2006, we calculate an average daily amount of 6,671 CFS. ${ }^{15}$ We abstract from fluctuations in water inflow over a typical day, and assume that inflow is a constant 6,671 CFS for each hour.

\footnotetext{
${ }^{15}$ This daily average excludes the months of April and May which are atypical with water inflows significantly higher than the rest of the year. When calculating the daily average, we also ignore the seasonal variation of water inflows over the years (excluding the months of April and May). The analysis for a specific season could be easily conducted by using the daily average corresponding to the season.
} 


\subsection{Modeling Hydropower Generation}

In this section, we specify the hydro power production function and the gross head function. The production function we adopt is standard in the power engineering literature, and is identical to that used in Philpott et al. [2000]. Water flowing through a turbine generates electricity by changing its potential energy into electrical energy. The amount of power available from a hydro power station is proportional to the product of its water flow rate, its water head and its generation efficiency. The hydro electricity generation function is determined empirically and is, in general, a non-linear function of the turbine discharge and the gross head. The amount of electricity produced by each unit (turbine) can be calculated using the following relation:

$$
q_{t}^{h}\left(r_{t}, w_{t}\right) \propto r_{t} h_{t}\left(r_{t}, w_{t}\right) e\left(r_{t}, h_{t}\right)
$$

where, $q_{t}^{h}$ is the power output, $r_{t}$ is the flow rate, $h_{t}$ is the gross head, $e$ is the efficiency factor and $\propto$ means proportion. Gross head refers to the vertical distance between the top of the penstock that conveys water under pressure and the point where the water discharges from the turbine. Here, the gross head is a function of the flow rate and the water content, and can be represented as $h_{t}\left(r_{t}, w_{t}\right)$. The generation efficiency in converting water flow to electrical power is a non-linear function of the flow rate and the gross head of the water flowing through the turbine, and can be written as $e\left(r_{t}, h_{t}\right)$. Due to the complexity of this nonseparable hydro production function, we have chosen to make a number of simplifying assumptions about its functional form for our model. Following Harpman [1999], Equation (2.25) becomes:

$$
q_{t}^{h}\left(r_{t}, h_{t}\left(w_{t}\right)\right)=0.001 g r_{t} h_{t}\left(w_{t}\right) e
$$

where, $g$ is the gravitational constant (32.15 feet-per-square-second) and the factor 0.001 converts $q_{t}^{h}$ to $\mathrm{MW}$ from KW, $r_{t}$ is in CFS and $h_{t}$ is in feet. According to Equation (2.26) gross head is only a function of the water content and does not vary with the flow rate, and the generation efficiency is kept as constant over the course of our (short-term) planning horizon (Hreinsson [1988]). Energy is always lost when converted from one form 
to another, and all the equipment used to convert power available in the flowing water to electrical power is less than 100 percent efficient. We use an efficiency factor of 0.87 . Therefore, the right hand side of Equation $(2.26)$ can be rewritten as $0.001 \times 32.15 \times 0.87 \times$ $r_{t} h_{t}\left(w_{t}\right)=0.028 r_{t} h_{t}\left(w_{t}\right)$ where $r_{t}$ is in CFS and $h_{t}$ is in feet. This simple formulation of the hydroelectric generating plant's production function has the characteristics of convexity, continuity and smoothness, which implies that standard optimization techniques can be usefully applied.

The level of head can be expressed as function of the water content in the reservoir. Due to the unavailability of some key data, following Edwards et al. [1999] we make the simplifying assumption of a linear functional form which can be written as:

$$
h_{t}\left(w_{t}\right)=\beta w_{t}
$$

where, $w_{t}$ is the water content in acre-feet. Then the parameter value of beta can be approximated using the available data. Under the normal operating range, the calibrated beta $^{16}$ value is 0.0089 . The advantage of using linear functional form is that only one parameter needs to be calibrated and it provides a good approximation when converting from gross head to reservoir storage, particularly for reservoirs with high inflows but small storage capacities.

\subsection{Empirical Analysis}

In this section, we examine three optimization cases under various operational and environmental constraints. The baseline case optimizes Equation (2.13) subject to Equations (2.14), (2.15), and Equations (2.19) through (2.24). The second case adds both the minimum and maximum water release requirements given by Equation (2.18). The third case adds extra up-ramping and down-ramping constraints, which are Equations (2.16)-(2.17).

\footnotetext{
${ }^{16}$ If the key data are available, we could specify a more realistic function form for Equation (2.27) and perform a goodness of fit test of the calibrated model.
} 
Hourly contract demand, given in Table 2.1, is based on the contract used in Edwards et al. [1999] but is scaled up to match the production capacity of our medium-sized prototype hydro station. For the baseline case the constraints are specified as follows:

- The minimum spill rate is 0 CFS and the maximum spill rate is 10,000 CFS;

- The minimum water content requirement is 7,000 acre-feet and the maximum value is 17,497 acre-feet;

- Hydro capacity varies from $0 \mathrm{MW}$ to $336 \mathrm{MW}$;

- A maximum of 13,100 acre-feet of water may be released during a 24 hour period for power generation;

For the case with release rate constraints the following restrictions are added to the baseline case:

- The minimum water release requirement is 2,000 CFS and the maximum release constraint is $15,000 \mathrm{CFS}$;

For the case with ramping rate constraints the following restrictions are added to the baseline case:

- Up-ramping and down-ramping constraints are 1,000 Cubic-feet-per-second per hour (CFS-hr);

- The minimum water release requirement is 2,000 CFS and the maximum release constraint is $15,000 \mathrm{CFS}$;

The constraint on the maximum quantity of water release during a 24 hour period for power generation may be thought of as an environmental constraint ensuring that the dam will not be drained in any period or it may be a technical constraint of the turbines. Note that this constraint on total release does not include spillage. Spillage will always be kept 
Table 2.1: Parameter Values Used in the Empirical Examples

\begin{tabular}{|c|c|c|c|c|}
\hline Hour & Time (Hour) & Demand (MW) & Water Inflow (CFS) & Price $(\$ / M W h)$ \\
\hline 0 & 10pm-11pm & 199 & 6671 & 62 \\
\hline 1st & 11pm-12pm & 159 & 6671 & 36 \\
\hline 2nd & 12pm-1am & 112 & 6671 & 36 \\
\hline $3 \mathrm{rd}$ & 1am-2am & 116 & 6671 & 36 \\
\hline 4 th & $2 \mathrm{am}-3 \mathrm{am}$ & 116 & 6671 & 36 \\
\hline 5th & $3 a m-4 a m$ & 114 & 6671 & 36 \\
\hline 6 th & 4am-5am & 125 & 6671 & 36 \\
\hline 7th & $5 \mathrm{am}-6 \mathrm{am}$ & 128 & 6671 & 36 \\
\hline 8th & 6am-7am & 134 & 6671 & 62 \\
\hline 9th & 7am-8am & 146 & 6671 & 62 \\
\hline 10th & 8am-9am & 164 & 6671 & 62 \\
\hline 11 th & 9am-10am & 181 & 6671 & 62 \\
\hline 12 th & 10am-11am & 199 & 6671 & 62 \\
\hline 13th & 11am-12am & 226 & 6671 & 62 \\
\hline 14 th & $12 \mathrm{am}-1 \mathrm{pm}$ & 267 & 6671 & 62 \\
\hline 15 th & $1 \mathrm{pm}-2 \mathrm{pm}$ & 291 & 6671 & 62 \\
\hline 16 th & $2 \mathrm{pm}-3 \mathrm{pm}$ & 314 & 6671 & 62 \\
\hline 17 th & $3 \mathrm{pm}-4 \mathrm{pm}$ & 336 & 6671 & 62 \\
\hline 18th & $4 \mathrm{pm}-5 \mathrm{pm}$ & 336 & 6671 & 62 \\
\hline 19 th & $5 \mathrm{pm}-6 \mathrm{pm}$ & 336 & 6671 & 62 \\
\hline 20 th & $6 \mathrm{pm}-7 \mathrm{pm}$ & 336 & 6671 & 62 \\
\hline $21 \mathrm{st}$ & $7 \mathrm{pm}-8 \mathrm{pm}$ & 336 & 6671 & 62 \\
\hline 22nd & 8pm-9pm & 291 & 6671 & 62 \\
\hline $23 r d$ & 9pm-10pm & 251 & 6671 & 62 \\
\hline 24 th & 10pm-11pm & 199 & 6671 & 62 \\
\hline
\end{tabular}

to a minimum as it does not contribute to profits. In the following examples the constraint on the maximum quantity of water release is set slightly below the total quantity of water inflow during the day implying that once the desired water level is obtained in the dam, it will be necessary to spill a certain amount each day to avoid overtopping. ${ }^{17}$ In practice the option to spill allows a hydro operator flexibility in cases where water inflow is higher than normal. This is not an issue in our empirical example in which water inflow does not vary from one hour to the next.

We begin the optimization by choosing an initial water release rate and water content. The optimization proceeds over the 5 day period. Through the choice of optimal hourly

\footnotetext{
${ }^{17}$ One CFS for 1 hour converts to approximated 0.082646 acre-feet. Converting the water inflow in each hour in Table 2.1 and adding over the 24 hour period gives 13,232 acre-feet as the total inflow.
} 
water releases, the water level in the dam changes over time until a steady state is reached so that subsequent days are identical to the previous day. The initial conditions are specified so that a steady state is reached (or nearly reached) within the five day optimization scenario. ${ }^{18}$ We report results for the fourth day for all cases. The first 7 hours of any day represent the off-peak period and the next 17 hours represent the on-peak period.

The results of the optimization are reported in the following sections. We begin with the baseline which uses the basic operational constraints, but no restrictions on minimum and maximum releases and ramping. The operational constraints are Equations (2.14) (water balance), (2.15) (contract demand) and (2.19)-(2.24), which are constraints on water spillage, head level, water content, hydro production, resale power, and total water release. Although our focus is on ramping constraints, we first consider the impact of minimum and maximum flow constraints alone and then add on ramping constraints.

\subsubsection{Baseline Optimization}

The base case results for a steady state day are reported in Table 2.2 and Figure 2.1. In Table 2.2, water release is seen to be zero during the off-peak hours. The spillage shown during off-peak periods is the amount needed to equalize flow into and out of the dam, so that the water level remains unchanged. During on-peak hours, water releases rise fairly steadily, peaking at 11,343 CFS at the 24th hour. The largest ramping-up in the water release rate occurs from the 7th to the 8th hour which is the cross over point from off-peak to on-peak. Similarly, the largest ramping-down occurs between the 10pm-11pm and $11 \mathrm{pm}-12 \mathrm{pm}$ periods of the day, which is at the cross point from the on-peak period to the off-peak period. During the off-peak period contract demand is satisfied only by purchasing power from the market for resale. During on-peak hours contract demand is met by hydro power only and for a significant number of hours more hydro electricity is produced than the contract requires. No thermal power is purchased for resale in this

\footnotetext{
${ }^{18} \mathrm{An}$ initial water release rate of 7,000 CFS is used in all cases. An initial water content of 14,000 acre-feet is used for the cases without ramping rate restrictions, while 17,000 acre-feet is used for the cases with ramping rate restrictions. The steady state is optimum since we are solving a convex optimization problem under the simplified assumptions for the hydro power production function.
} 
Table 2.2: Baseline Experiment

\begin{tabular}{|c|c|c|c|c|c|c|c|}
\hline Hour & Time (Hour) & $\begin{array}{l}\text { Water Con- } \\
\text { tent (Acre- } \\
\text { feet) }\end{array}$ & $\begin{array}{l}\text { Spillway } \\
\text { (CFS) }\end{array}$ & $\begin{array}{l}\text { Water } \\
\text { Release } \\
\text { (CFS) }\end{array}$ & $\begin{array}{l}\text { Hydro } \\
\text { Generation } \\
(\mathrm{MW})\end{array}$ & $\begin{array}{l}\text { Power } \\
\text { Purchase } \\
(\mathrm{MW})\end{array}$ & $\begin{array}{l}\text { Total } \\
\text { Power } \\
\text { (MW) }\end{array}$ \\
\hline 0 & 10pm-11pm & 13768 & $\mathrm{~N} / \mathrm{A}$ & $\mathrm{N} / \mathrm{A}$ & $\mathrm{N} / \mathrm{A}$ & $\mathrm{N} / \mathrm{A}$ & $\mathrm{N} / \mathrm{A}$ \\
\hline 1 st & $11 \mathrm{pm}-12 \mathrm{pm}$ & 14307 & 157 & 0 & 0 & 159 & 159 \\
\hline 2nd & 12pm-1am & 14817 & 490 & 0 & 0 & 112 & 112 \\
\hline $3 r d$ & 1am-2am & 15357 & 146 & 0 & 0 & 116 & 116 \\
\hline 4 th & $2 \mathrm{am}-3 \mathrm{am}$ & 15888 & 236 & 0 & 0 & 116 & 116 \\
\hline 5 th & $3 \mathrm{am}-4 \mathrm{am}$ & 16433 & 85 & 0 & 0 & 114 & 114 \\
\hline 6th & $4 \mathrm{am}-5 \mathrm{am}$ & 16947 & 451 & 0 & 0 & 125 & 125 \\
\hline 7th & $5 \mathrm{am}-6 \mathrm{am}$ & 17497 & 10 & 0 & 0 & 128 & 128 \\
\hline 8th & 6am-7am & 17497 & 0 & 6671 & 251 & 0 & 251 \\
\hline 9th & 7am-8am & 17497 & 0 & 6671 & 251 & 0 & 251 \\
\hline 10 th & 8am-9am & 17497 & 0 & 6671 & 251 & 0 & 251 \\
\hline 11th & $9 \mathrm{am}-10 \mathrm{am}$ & 17375 & 0 & 8147 & 304 & 0 & 304 \\
\hline 12 th & 10am-11am & 17175 & 0 & 9093 & 336 & 0 & 336 \\
\hline 13 th & 11am-12am & 16966 & 0 & 9205 & 336 & 0 & 336 \\
\hline 14 th & $12 \mathrm{am}-1 \mathrm{pm}$ & 16746 & 0 & 9326 & 336 & 0 & 336 \\
\hline 15 th & $1 \mathrm{pm}-2 \mathrm{pm}$ & 16516 & 0 & 9456 & 336 & 0 & 336 \\
\hline 16th & $2 \mathrm{pm}-3 \mathrm{pm}$ & 16275 & 0 & 9596 & 336 & 0 & 336 \\
\hline 17 th & $3 \mathrm{pm}-4 \mathrm{pm}$ & 16020 & 0 & 9748 & 336 & 0 & 336 \\
\hline 18th & $4 \mathrm{pm}-5 \mathrm{pm}$ & 15752 & 0 & 9914 & 336 & 0 & 336 \\
\hline 19th & $5 \mathrm{pm}-6 \mathrm{pm}$ & 15469 & 0 & 10095 & 336 & 0 & 336 \\
\hline 20 th & $6 \mathrm{pm}-7 \mathrm{pm}$ & 15170 & 0 & 10295 & 336 & 0 & 336 \\
\hline $21 \mathrm{st}$ & $7 \mathrm{pm}-8 \mathrm{pm}$ & 14853 & 0 & 10515 & 336 & 0 & 336 \\
\hline 22nd & $8 \mathrm{pm}-9 \mathrm{pm}$ & 14515 & 0 & 10759 & 336 & 0 & 336 \\
\hline $23 r d$ & $9 \mathrm{pm}-10 \mathrm{pm}$ & 14154 & 0 & 11033 & 336 & 0 & 336 \\
\hline 24th & $10 \mathrm{pm}-11 \mathrm{pm}$ & 13768 & 0 & 11343 & 336 & 0 & 336 \\
\hline
\end{tabular}

period. Clearly, in this case the absence of minimum and maximum release constraints and ramping constraints allows for rather dramatic changes in water release rates. Figure 2.1 plots the total power (hydro production and resale power), contract demand, hydroelectric production and power purchase for resale under this baseline case. It shows a clear pattern of hydro-shifting. 


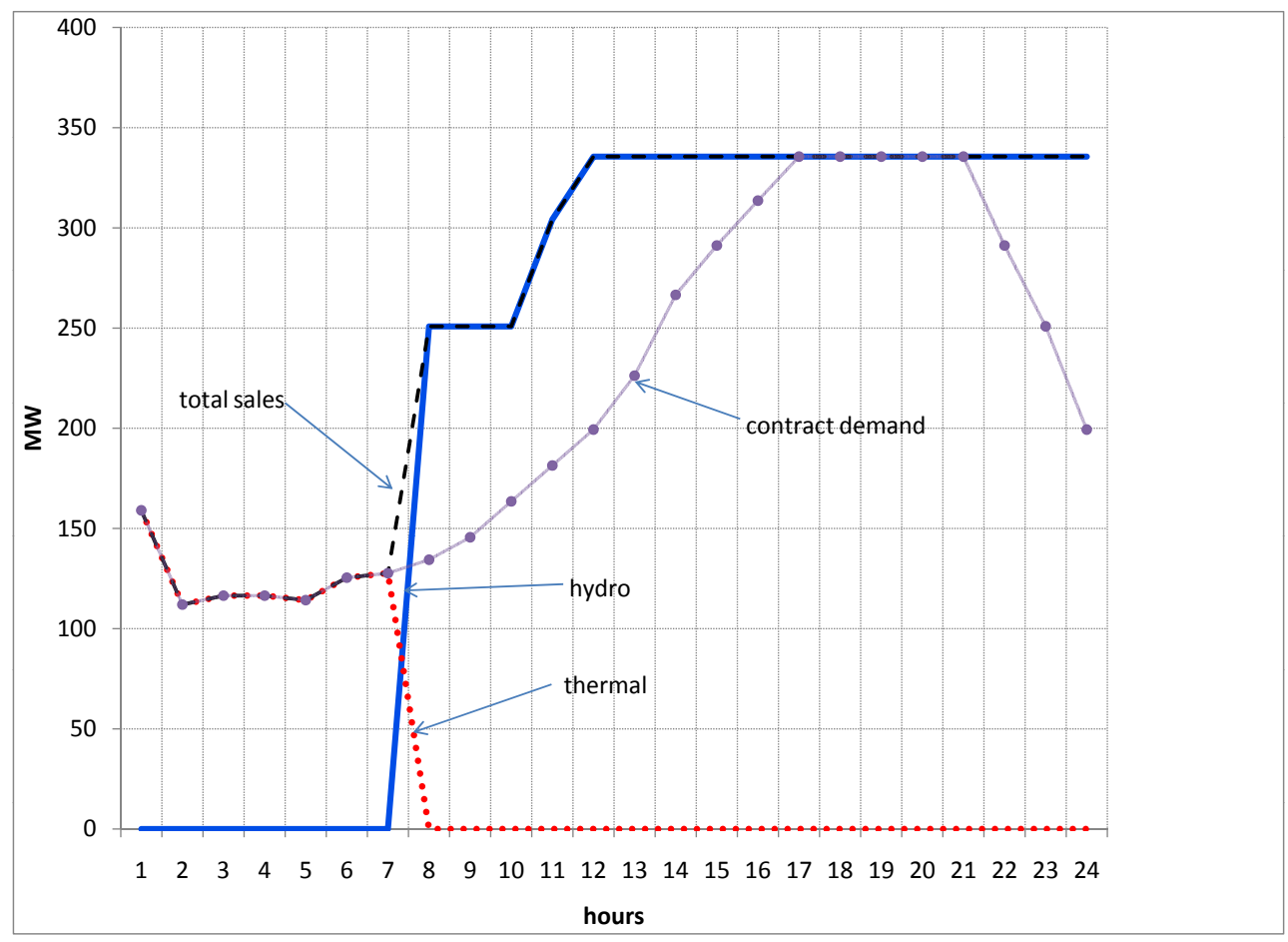

Figure 2.1: Hydro and Thermal Power Production: Baseline Experiment

These results make sense intuitively and are consistent with Edwards et al. [1999]. Because of the upper limit on the total water flow through the turbine in any one day, it is in the interests of the hydro operator to release the permitted water flow when the electricity price is highest, which is during on-peak hours. In addition, the requirement that sufficient power must be sold during the base period in order to satisfy the demand requirement, induces the decision maker to purchase and resell thermal power during the off-peak period to satisfy this contract demand. To maximize the value of hydro resources, the power station stores water during the off-peak period for release during the on-peak period, and sells thermal power during the base period to satisfy the demand requirement of its customers. 


\subsubsection{Optimization with Release Rate Constraints}

In this second optimization, we impose a minimum release requirement of 2,000 CFS and a maximum release requirement of $15,000 \mathrm{CFS}$. Together with the operational constraints in the first scenario, these constraints cause several major changes in the water release profile during the representative day (Table 2.3 and Figure 2.2). First, off-peak period releases increase to satisfy the new minimum release rate constraint and are maintained at the lower bound of 2,000 CFS from the 1st hour up to the 7th hour of the representative day. As a result, from the 8 th hour to the 24 th hour water releases are either slightly lower than or the same as the baseline case. This indicates that, during the off-peak period, it is optimal to maintain the minimum release rate and to purchase from the spot market the remaining power needed to meet the demand requirement. During the on-peak period, since the electricity prices are much higher, it is desirable to keep the water release rate similar to the baseline case.

During the off-peak period, at a release rate of 2,000 CFS the produced hydro power is lower than the contract demand, however during the on-peak period the hydro power production is either higher than or equal to the contract demand. Correspondingly, power resales are lower during the off-peak hours than under the baseline case. As in the baseline case, there are no power resales in the on-peak period.

Also consistent with the baseline case, up-ramping is highest from the 7th to the 8th hour and down-ramping is highest from 10pm-11pm to $11 \mathrm{pm}-12 \mathrm{pm}$. However, these ramping rate peaks have lower magnitudes than in the baseline case. As shown in Table 2.3, the maximum release constraint is never binding during the on-peak period. Maximum water release occurs from $10 \mathrm{pm}-11 \mathrm{pm}$, and at 10,463 CFS is less that in the baseline case. After imposing the minimum and maximum release constraints, hydro-shifting is still apparent, but less significant compared with the baseline case, as is illustrated in Figure 2.2. This indicates that these extra environmental constraints limit the station's ability to make full use of the benefit of hydro-shifting, and therefore reduce its value. 
Table 2.3: Case Including Minimum and Maximum Release Constraints

\begin{tabular}{|c|c|c|c|c|c|c|c|}
\hline Hour & Time (Hour) & $\begin{array}{l}\text { Water Con- } \\
\text { tent (Acre- } \\
\text { feet) }\end{array}$ & $\begin{array}{l}\text { Spillway } \\
\text { (CFS) }\end{array}$ & $\begin{array}{l}\text { Water } \\
\text { Release } \\
\text { (CFS) }\end{array}$ & $\begin{array}{l}\text { Hydro } \\
\text { Generation } \\
(\mathrm{MW})\end{array}$ & $\begin{array}{l}\text { Power } \\
\text { Purchase } \\
(\mathrm{MW})\end{array}$ & $\begin{array}{l}\text { Total } \\
\text { Power } \\
\text { (MW) }\end{array}$ \\
\hline 0 & 10pm-11pm & 14925 & $\mathrm{~N} / \mathrm{A}$ & $\mathrm{N} / \mathrm{A}$ & $\mathrm{N} / \mathrm{A}$ & $\mathrm{N} / \mathrm{A}$ & $\mathrm{N} / \mathrm{A}$ \\
\hline 1 st & $11 \mathrm{pm}-12 \mathrm{pm}$ & 15311 & 0 & 2000 & 66 & 93 & 159 \\
\hline 2nd & 12pm-1am & 15697 & 0 & 2000 & 67 & 45 & 112 \\
\hline $3 r d$ & 1am-2am & 16083 & 0 & 2000 & 69 & 47 & 116 \\
\hline 4 th & 2am-3am & 16469 & 0 & 2000 & 71 & 46 & 117 \\
\hline 5 th & $3 a m-4 a m$ & 16855 & 0 & 2000 & 72 & 42 & 114 \\
\hline 6 th & $4 \mathrm{am}-5 \mathrm{am}$ & 17241 & 0 & 2000 & 74 & 51 & 125 \\
\hline 7th & $5 \mathrm{am}-6 \mathrm{am}$ & 17497 & 1574 & 2000 & 75 & 52 & 127 \\
\hline 8th & 6am-7am & 17497 & 0 & 6671 & 251 & 0 & 251 \\
\hline 9th & 7am-8am & 17497 & 0 & 6671 & 251 & 0 & 251 \\
\hline 10 th & 8am-9am & 17497 & 0 & 6671 & 251 & 0 & 251 \\
\hline 11th & 9am-10am & 17497 & 0 & 6671 & 251 & 0 & 251 \\
\hline 12 th & 10am-11am & 17497 & 0 & 6671 & 251 & 0 & 251 \\
\hline 13 th & 11am-12am & 17497 & 0 & 6671 & 251 & 0 & 251 \\
\hline 14 th & $12 \mathrm{am}-1 \mathrm{pm}$ & 17419 & 0 & 7621 & 285 & 0 & 285 \\
\hline 15 th & $1 \mathrm{pm}-2 \mathrm{pm}$ & 17220 & 0 & 9069 & 336 & 0 & 336 \\
\hline 16th & $2 \mathrm{pm}-3 \mathrm{pm}$ & 17013 & 0 & 9179 & 336 & 0 & 336 \\
\hline 17 th & $3 \mathrm{pm}-4 \mathrm{pm}$ & 16796 & 0 & 9298 & 336 & 0 & 336 \\
\hline 18th & $4 \mathrm{pm}-5 \mathrm{pm}$ & 16569 & 0 & 9426 & 336 & 0 & 336 \\
\hline 19th & $5 \mathrm{pm}-6 \mathrm{pm}$ & 16330 & 0 & 9564 & 336 & 0 & 336 \\
\hline 20 th & $6 \mathrm{pm}-7 \mathrm{pm}$ & 16078 & 0 & 9713 & 336 & 0 & 336 \\
\hline $21 \mathrm{st}$ & $7 \mathrm{pm}-8 \mathrm{pm}$ & 15813 & 0 & 9876 & 336 & 0 & 336 \\
\hline 22nd & 8pm-9pm & 15534 & 0 & 10053 & 336 & 0 & 336 \\
\hline $23 \mathrm{rd}$ & $9 \mathrm{pm}-10 \mathrm{pm}$ & 15238 & 0 & 10248 & 336 & 0 & 336 \\
\hline 24 th & $10 \mathrm{pm}-11 \mathrm{pm}$ & 14925 & 0 & 10463 & 336 & 0 & 336 \\
\hline
\end{tabular}




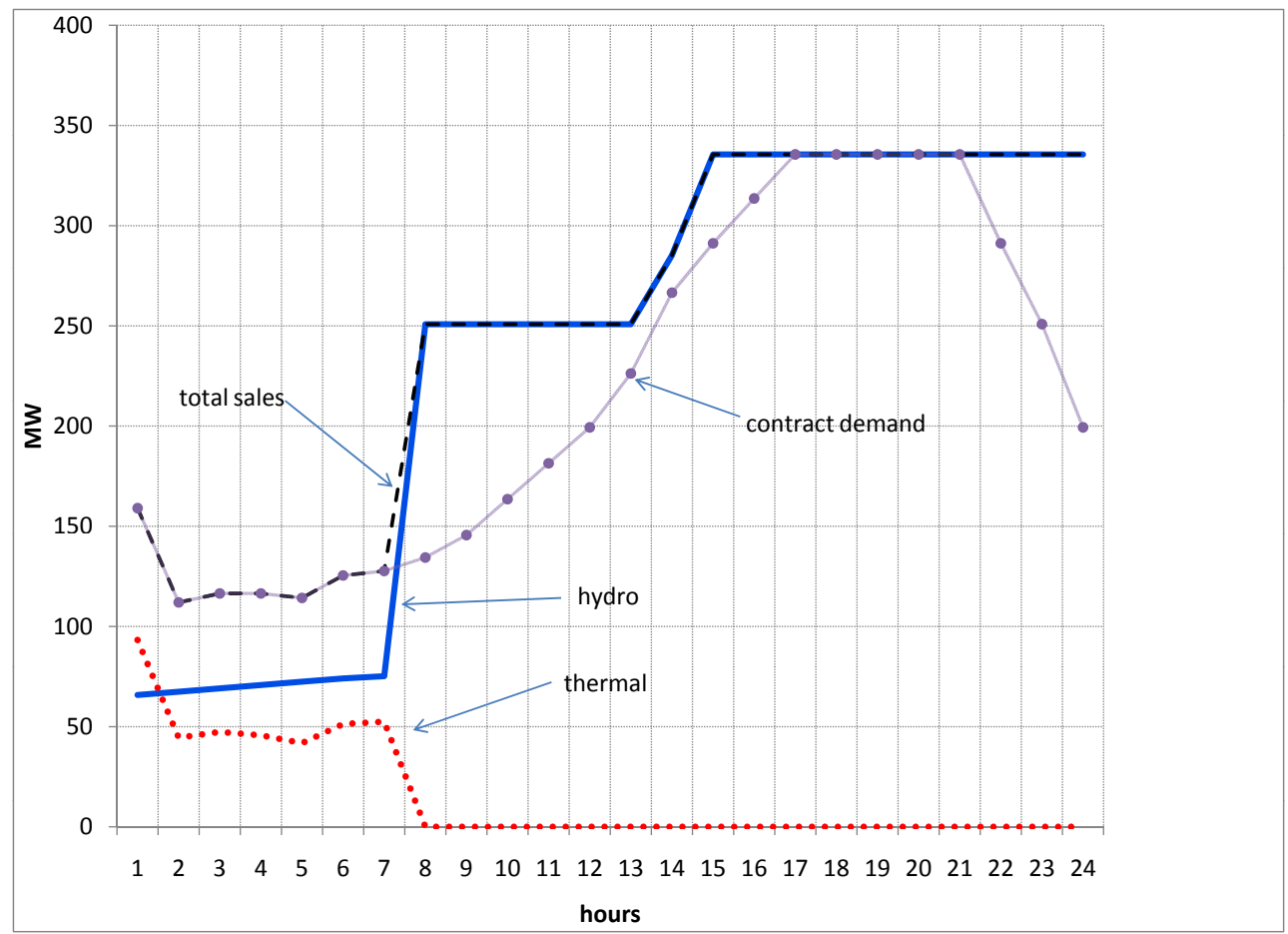

Figure 2.2: Hydro and Thermal Power Production: Experiment with Minimum and Maximum Release Constraints 


\subsubsection{Optimization with Ramping Rate Constraints}

In this scenario we add up-ramping and down-ramping restrictions, both of which are set initially at 1,000 CFS-hr. As Table 2.4 and Figure 2.3 illustrate, with ramping restrictions the highest on-peak water release rate during the representative day reaches the maximum of 9,621 CFS in the 20th hour, which is lower than the previous two cases. The ramping constraints reduce the extent of hydro-shifting that is possible. In the off-peak period, the hydro power station gradually ramps down the water release rate, and then in the on-peak period gradually ramps up again. However while still in the on-peak period, after the 20th hour, ramping down has commenced in preparation for the approaching off-peak period.

The change in the pattern of hydro production over the day compared to the previous scenarios is most easily seen in Figure 2.3 compared to Figure 2.2. With the maximum allowable release during a day, the increase in hydro production during the off-peak period implies there will be a reduction during the on-peak period. During the 21st and 22nd hours a small portion of demand is met by purchases of thermal power. The inability to fully satisfy peak demand with hydro production will have a negative effect on profits.

\subsubsection{Comparing the Three Optimization Scenarios}

Figure 2.4 compares water release rates for these three optimization scenarios. From this graph the shifting of hydro production from the on-peak to the off-peak period is very evident for the case with minimum and maximum flow constraints compared to the baseline. This shift is even larger for the case with ramping constraints. 
Table 2.4: Case Including Up-ramping and Down-ramping Constraints

\begin{tabular}{|c|c|c|c|c|c|c|c|}
\hline Hour & Time (Hour) & $\begin{array}{l}\text { Water Con- } \\
\text { tent (Acre- } \\
\text { feet) }\end{array}$ & $\begin{array}{l}\text { Spillway } \\
\text { (CFS) }\end{array}$ & $\begin{array}{l}\text { Water } \\
\text { Release } \\
\text { (CFS) }\end{array}$ & $\begin{array}{l}\text { Hydro } \\
\text { Generation } \\
(\mathrm{MW})\end{array}$ & $\begin{array}{l}\text { Power } \\
\text { Purchase } \\
(\mathrm{MW})\end{array}$ & $\begin{array}{l}\text { Total } \\
\text { Power } \\
\text { (MW) }\end{array}$ \\
\hline 0 & 10pm-11pm & 15876 & $\mathrm{~N} / \mathrm{A}$ & 6490 & $\mathrm{~N} / \mathrm{A}$ & $\mathrm{N} / \mathrm{A}$ & $\mathrm{N} / \mathrm{A}$ \\
\hline 1 st & $11 \mathrm{pm}-12 \mathrm{pm}$ & 15974 & 0 & 5490 & 188 & 0 & 188 \\
\hline 2nd & 12pm-1am & 16154 & 0 & 4490 & 156 & 0 & 156 \\
\hline $3 r d$ & 1am-2am & 16417 & 0 & 3490 & 123 & 0 & 123 \\
\hline 4 th & 2am-3am & 16762 & 0 & 2490 & 90 & 27 & 117 \\
\hline 5 th & $3 a m-4 a m$ & 17093 & 0 & 2671 & 98 & 16 & 114 \\
\hline 6 th & $4 \mathrm{am}-5 \mathrm{am}$ & 17341 & 0 & 3671 & 137 & 0 & 137 \\
\hline 7th & $5 \mathrm{am}-6 \mathrm{am}$ & 17497 & 108 & 4671 & 176 & 0 & 176 \\
\hline 8th & 6am-7am & 17497 & 1000 & 5671 & 213 & 0 & 213 \\
\hline 9th & 7am-8am & 17497 & 0 & 6671 & 251 & 0 & 251 \\
\hline 10th & 8am-9am & 17497 & 0 & 6671 & 251 & 0 & 251 \\
\hline 11th & 9am-10am & 17497 & 0 & 6671 & 251 & 0 & 251 \\
\hline 12 th & 10am-11am & 17497 & 0 & 6671 & 251 & 0 & 251 \\
\hline 13 th & 11am-12am & 17497 & 0 & 6671 & 251 & 0 & 251 \\
\hline 14 th & $12 \mathrm{am}-1 \mathrm{pm}$ & 17461 & 0 & 7110 & 267 & 0 & 267 \\
\hline 15 th & $1 \mathrm{pm}-2 \mathrm{pm}$ & 17342 & 0 & 8110 & 302 & 0 & 302 \\
\hline 16th & $2 \mathrm{pm}-3 \mathrm{pm}$ & 17140 & 0 & 9110 & 336 & 0 & 336 \\
\hline 17 th & $3 \mathrm{pm}-4 \mathrm{pm}$ & 16929 & 0 & 9225 & 336 & 0 & 336 \\
\hline 18th & $4 \mathrm{pm}-5 \mathrm{pm}$ & 16708 & 0 & 9347 & 336 & 0 & 336 \\
\hline 19th & $5 \mathrm{pm}-6 \mathrm{pm}$ & 16476 & 0 & 9478 & 336 & 0 & 336 \\
\hline 20 th & $6 \mathrm{pm}-7 \mathrm{pm}$ & 16233 & 0 & 9621 & 336 & 0 & 336 \\
\hline $21 \mathrm{st}$ & $7 \mathrm{pm}-8 \mathrm{pm}$ & 16029 & 0 & 9134 & 315 & 21 & 336 \\
\hline 22nd & $8 \mathrm{pm}-9 \mathrm{pm}$ & 15908 & 0 & 8134 & 278 & 13 & 291 \\
\hline $23 \mathrm{rd}$ & $9 \mathrm{pm}-10 \mathrm{pm}$ & 15870 & 0 & 7134 & 243 & 8 & 251 \\
\hline 24 th & $10 \mathrm{pm}-11 \mathrm{pm}$ & 15915 & 0 & 6134 & 210 & 0 & 210 \\
\hline
\end{tabular}




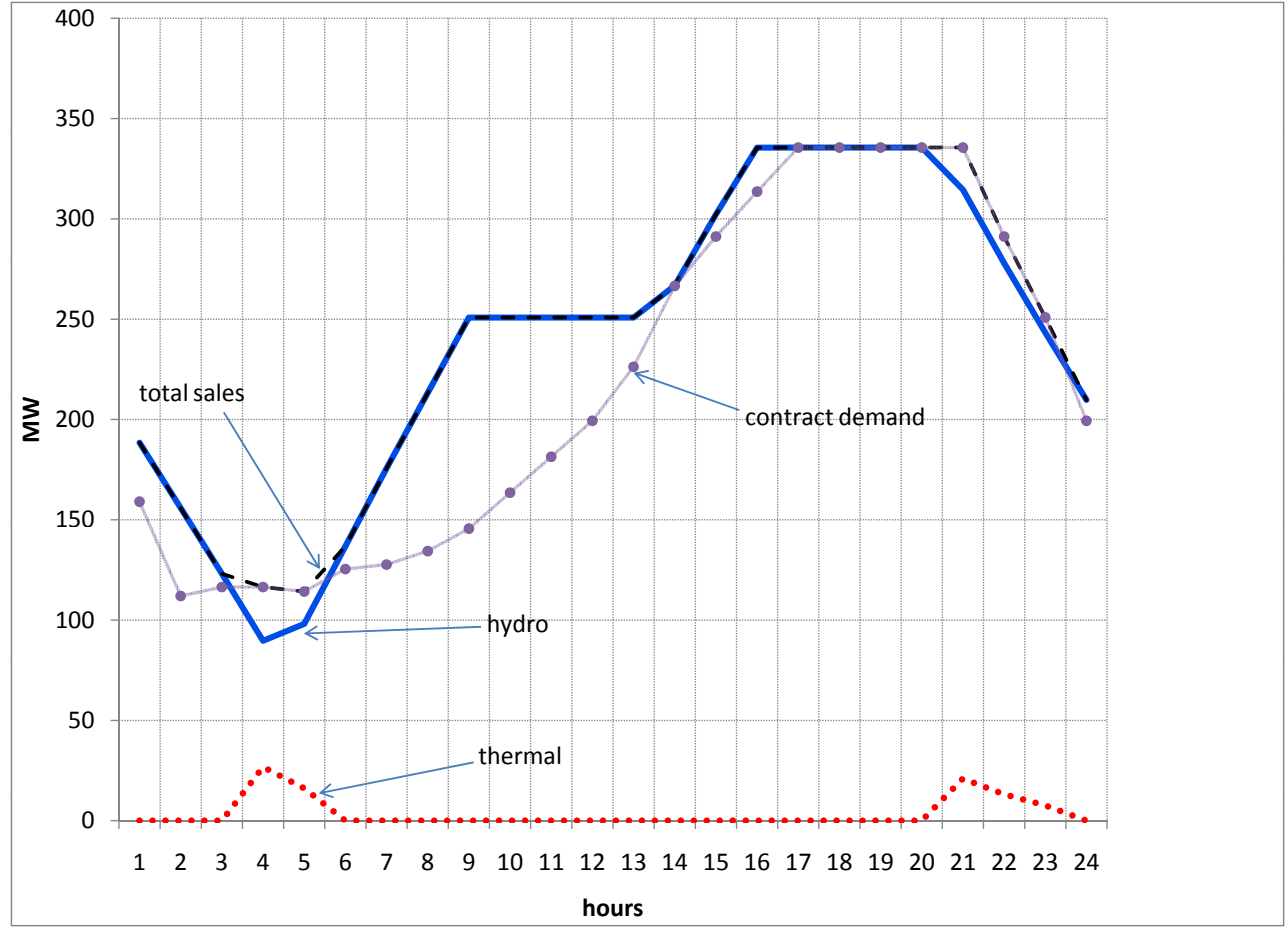

Figure 2.3: Hydro and Thermal Power Production: Experiment with Up-ramping and Down-ramping Constraints 


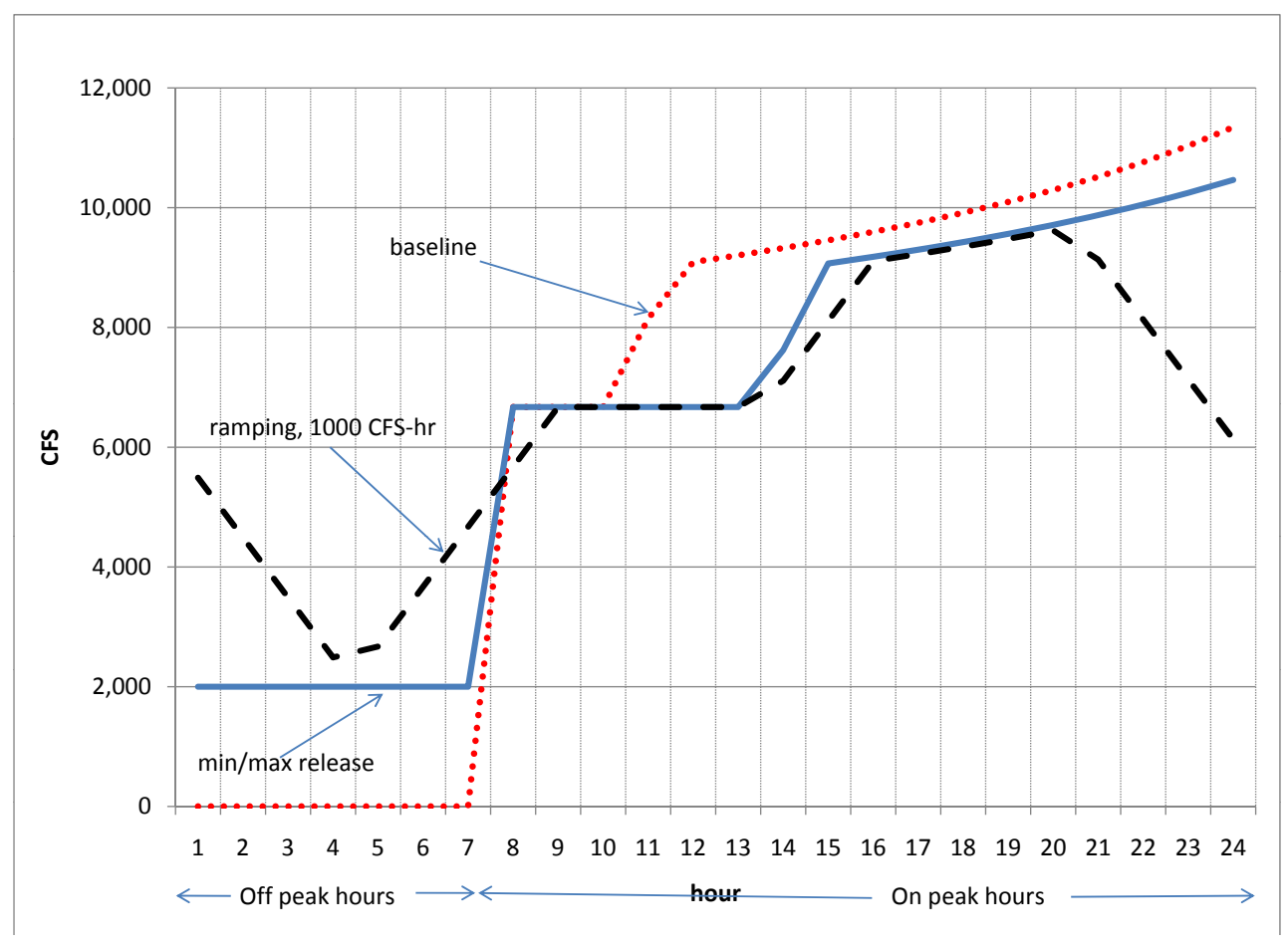

Figure 2.4: Comparing Water Release Rates for Hydro Production

The impact of these constraints on profits for a range of ramping constraints is detailed in Table 2.5 and Figure $2.5^{19}$. In general, the more restrictive the constraint the greater is the limitation on the station's operational flexibility and the larger the impact on profits. Under the baseline case, the total profit from providing power is $\$ 226$ thousand. For Case I with the minimum and maximum release constraints, the total daily profit drops to $\$ 223$ thousand, representing a $1.1 \%$ reduction over the baseline scenario. When ramping constraints of 5,000 CSF-hr are added in Case II profit drops marginally to $\$ 222$ thousand

\footnotetext{
${ }^{19}$ Case I is min/max release constraints only. Cases II through VIII include min/max release constraints as well as equal up and down ramping constraints respectively in CFS-hr of 5,000, 4,000, 3,000, 2,000, $1,000,500$, and 250. The percentage change of total profit is shown in Table 2.5.
} 
which is $1.7 \%$ less than the baseline. As ramping rate restrictions are increased profits continue to drop, until at a restriction of $250 \mathrm{CSF}-\mathrm{hr}$, i.e., the water release rate can increase or decrease by at most 250 CFS between any two consecutive hours, then the total profit drops to as low as $\$ 208$ thousand, which is an $8 \%$ decrease relative to the baseline. From Figure 2.5 we also observe profits fall proportionately more as ramping restrictions are increased when ramping rates are already quite restrictive - i.e. for rates of less than 2,000 CFS-hr ${ }^{20}$.

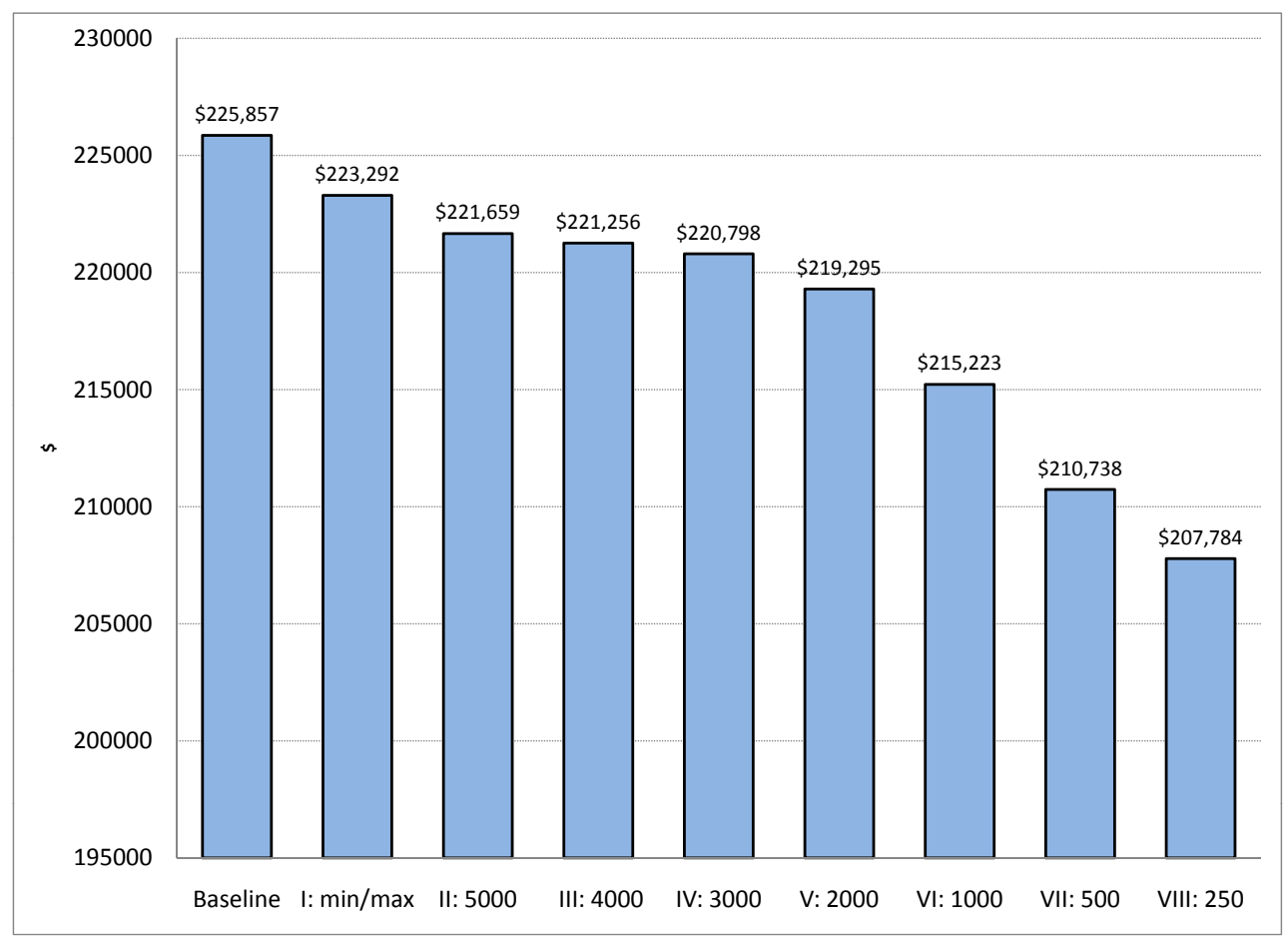

Figure 2.5: Comparing Profit Levels

\footnotetext{
${ }^{20}$ This could be viewed as the threshold between the sensitive and insensitive regions for the impact of ramping constraints on profits.
} 


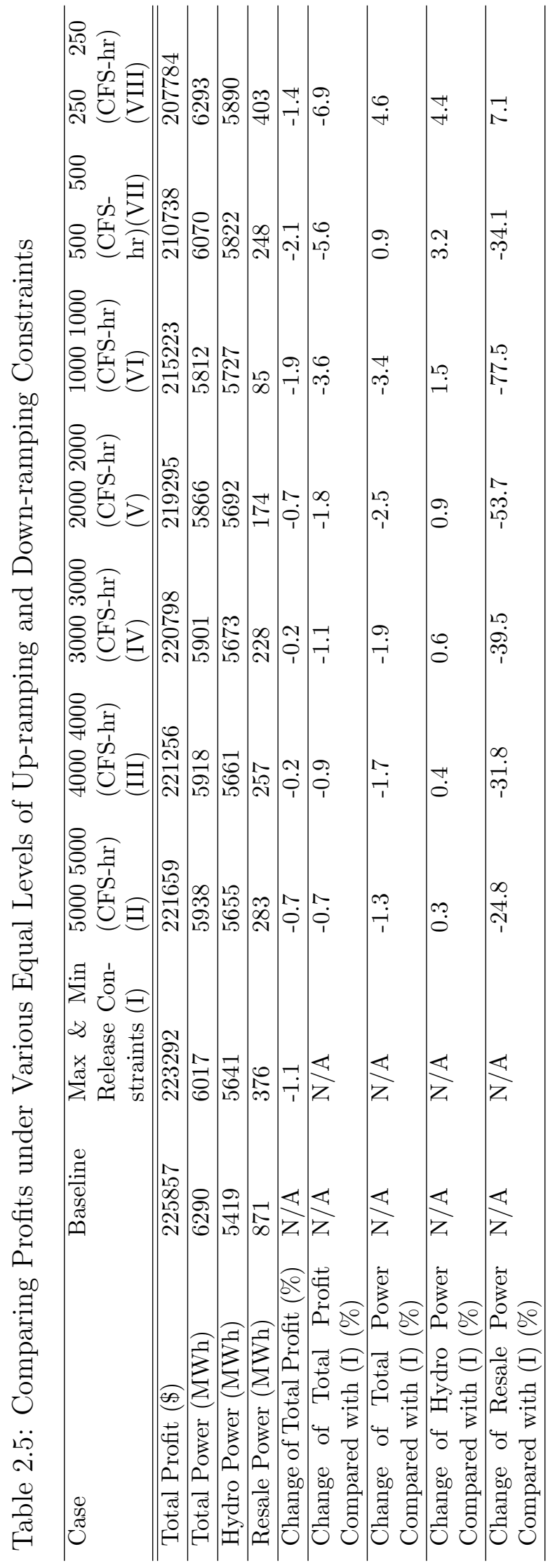




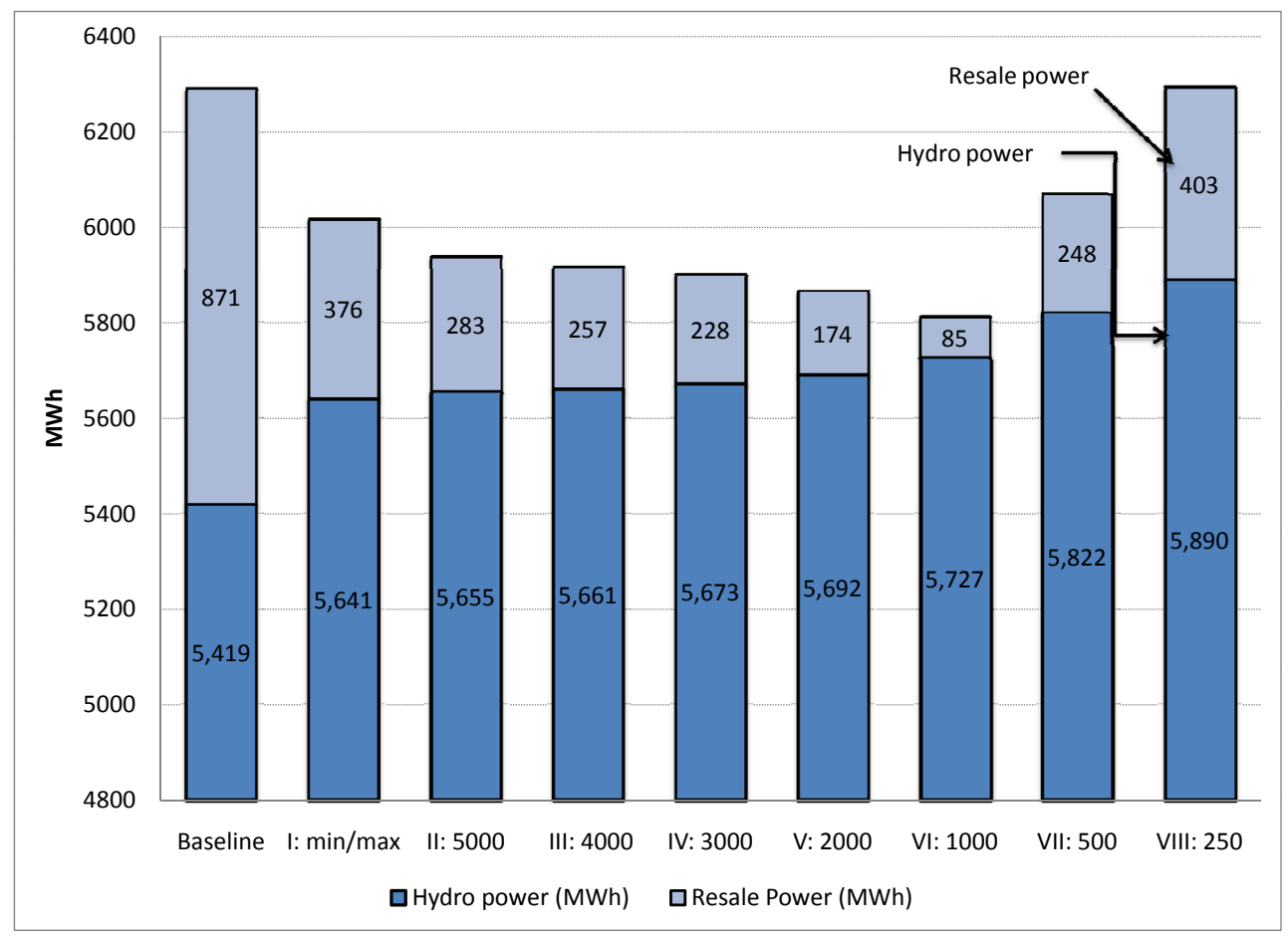

Figure 2.6: Comparing Power Production Levels 


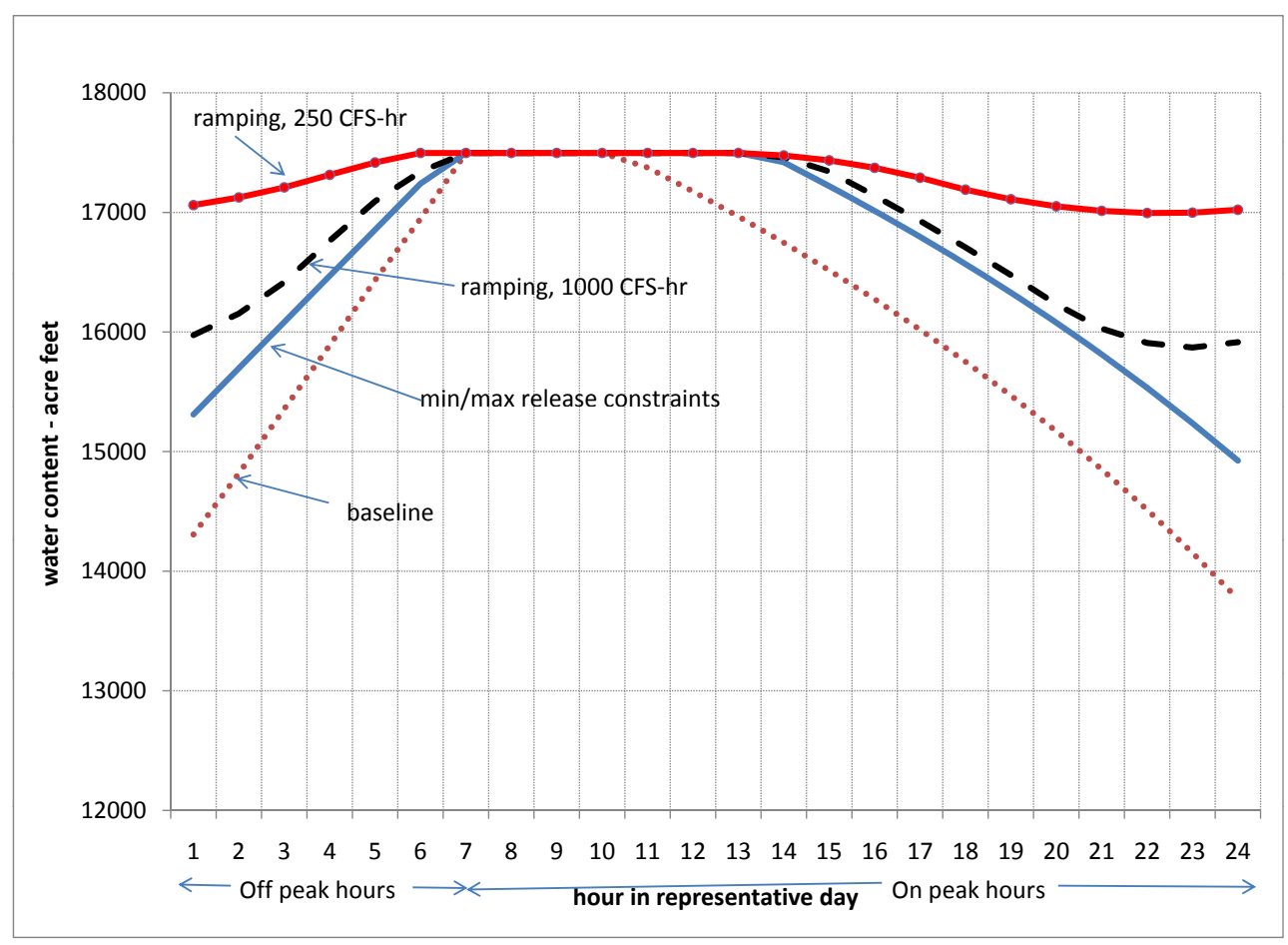

Figure 2.7: Comparing Water Content across Cases

In Table 2.5 and Figure $2.6^{21}$, we report total power sales, hydro power generation, and spot purchases for resale. Interestingly we observe that total power sales are affected, implying that the impact of the restrictions is not simply a redistribution from on-peak to off-peak periods. We observe the largest power production level in the most restrictive case (ramping rate constraints of $250 \mathrm{CFS}-\mathrm{hr}$ ). Figure 2.7 gives a clue as to why this is so. In this figure water content by hour is shown for the baseline case, the case of $\mathrm{min} / \mathrm{max}$ release constraints and the cases of 250 and 1,000 CFS-hr up and down ramping restrictions. The

\footnotetext{
${ }^{21}$ Case I is min/max release constraints only. Cases II through VIII include min/max release constraints as well as equal up and down ramping constraints respectively in CFS-hr of 5,000, 4,000, 3,000, 2,000, $1,000,500$, and 250 .
} 
flat portions on the graph are periods when water content is at the upper limit. Starting at a given initial water content, as optimization proceeds over the 5 day period the optimal choice of water release and spillage affects the water content of the dam. By the time a steady state is achieved, the water level profile by hour over a 24 hour period remains the same from one day to the next and the amount of spillage in one day is chosen so that the water level in the dam is maintained.

We observe from Figure 2.7 that the optimal water levels for the restricted cases (min/max release constraints and ramping rate constraints) are greater than or equal to the water level for the baseline case. Further, the ramping rate case shows a higher water level than the min/max release constraint case for many hours of the day. The larger water content is a result of optimal choices necessitated by the restrictions and allows the operator to generate more power with a given water release rate (recall Equation 2.26). This is needed in order to produce as much as possible during on-peak periods despite the ramping constraints.

In Figure 2.6 different levels of restrictions can also be seen to have an impact on the level of power purchased for resale. The largest amount of resale power occurs in the base case without restrictions. Resale power is reduced as ramping restrictions are made more restrictive moving from 5,000 CFS-hr to 1,000 CFS-hr. However for restrictions of 500 and 250 CFS-hr an increase in the purchase of resale power is observed. This purchase happens in peak period hours, and without it the hydro operator would be unable to meet contract demand. In summary, we observe that the ramping restrictions have caused an increased reliance on hydro-power ${ }^{22}$ and a decreased reliance on purchases for resale compared to the baseline case. This is a somewhat counter intuitive result.

The results we have shown so far for our prototype Ontario dam are consistent with Edwards et al. [1999]. Edwards showed that ramping restrictions increase the amount of hydro sold in the off-peak period and reduce it in the on-peak period and thereby reduce overall profitability of the hydro operations. Our results differ from Edwards in the finding that total hydro production may increase as ramping restrictions are imposed. This follows

\footnotetext{
${ }^{22}$ Ramping restrictions cause an increase of the water content compared to the baseline case (Figure 2.7 ), therefore the hydro power generation also increases over a 24 hour period.
} 
from our assumption that total hydro sales can exceed contract demand, and that as the hydro operator optimizes water releases over several days the water level in the dam adjusts until a steady state is reached. The ability to increase hydro production is a means for the operator to reduce the impact of ramping restrictions on profits. However, profitability is still affected with the most significant effects coming when ramping is restricted to 1,000 CFS-hr and less.

\subsection{Including the Environmental Impact of Changes in Thermal Generation}

In the previous sections, we detailed the impact of ramping restrictions on the profitability of the firm. However in setting a ramping rate policy a regulator should consider other potential impacts that will affect the public good. Ideally the determination of an optimal level of ramping constraints would begin with a comprehensive environmental assessment of the positive effect of various levels of ramping restrictions on the river ecosystem. Evidence on the benefits for the aquatic ecosystem would be weighed against the negative effects on hydro station profits as well as the environmental impact of the change in reliance on other sources of power generation such as fossil fuels. If one were able to put a dollar value on each of these effects, the optimal ramping rate restrictions could be chosen. However, practically it is very difficult to measure the environmental effect of ramping on the river ecosystem based on biological studies and it is even more challenging to calculate this effect quantitatively in terms of monetary value. Currently, there is very limited research on this environmental effect and no studies available to provide some appropriate monetary measure of the environmental benefit of ramping restrictions. In contrast, there are estimates available of the environmental costs of thermal power generation. In this section we estimate the difference in environmental damages due to the change in reliance on thermal generation as a result of ramping rate restrictions and add this to the loss in hydro profits to get an estimate of the total cost of ramping restrictions.

In this section we investigate the impact of imposing up-ramping and down-ramping 
restrictions when minimum and maximum flow restrictions are already in place. We will measure the unit environmental cost $(\$ / M W h)$ of the replacement power by using an estimate of the marginal external cost of emissions of a thermal generation plant. These emissions include $\mathrm{SO}_{2}, \mathrm{NO}_{x}$ and $\mathrm{CO}_{2}$. We consider two cases for thermal (replacement) power: (i) replacement power is generated with coal during both the off-peak and on-peak periods; or (ii) it is generated with coal during the off-peak period and natural gas during the on-peak period ${ }^{23}$.

In the empirical results presented in Section 2.7, we found that in the steady state, when ramping constraints were imposed, hydro production decreased during on-peak periods and increased during off-peak periods. Overall total hydro power production increased in the 24 hour period as ramping restrictions became increasingly tight. We assume that total market demand and production for electricity are not affected by the hydro power plant's operation. It follows that every unit change in hydro power production will be exactly offset by a change in thermal power generation. As a consequence, these flow restrictions will result in a decrease in polluting emissions from thermal power during off-peak hours and an increase in on-peak hours. Overall on a daily basis we will observe a reduction in pollutant emissions from thermal power.

For the first case, this environmental benefit is calculated as the total net increase in hydro production over the 24 hour period after imposing the ramping restrictions (which also equals the change in the amount of thermal power) multiplied by the marginal external costs of emissions for coal. For the second case, the associated total environmental benefit is calculated as the increase in the amount of hydro power generation during the off-peak period after imposing the ramping restrictions multiplied the marginal external costs of emissions for coal, minus the reduced amount of hydro power generation during the on-peak period after imposing the ramping restrictions multiplied by the marginal external costs of emissions for natural gas. The benefit of emissions reduction from thermal generation is subtracted from the lost profit caused by ramping restrictions which gives the net cost

\footnotetext{
${ }^{23}$ In Kotchen et al. [2006], they assume that the thermal (replacement) power during peak periods is generated with fuel oil and natural gas, while thermal power during off-peak periods is generated with coal only.
} 
of the ramping restrictions, ignoring any benefits that accrue to the aquatic ecosystem. This cost estimate provides a lower bound on the level of benefits to the aquatic ecosystem which would make ramping constraints worthwhile.

We consider the benefits of reduced emissions from thermal plants for the scenarios examined in the previous section of equal levels of up-ramping and down-ramping constraints. For the marginal external costs of emissions, we choose both the high and low cost estimates. $^{24}$ For coal, these are $67.18 \$ / \mathrm{MWh}$ and $45.20 \$ / \mathrm{MWh}$. For natural gas, these are $9.96 \$ / \mathrm{MWh}$ and $7.44 \$ / \mathrm{MWh}$.

The results for the scenario with coal as replacement power under various equal levels of up-ramping and down-ramping constraints are reported in Figure $2.8^{25}$. As we can observe, at any given level of ramping restrictions, higher marginal external costs of emissions always result in higher associated environmental benefits. However the cost in terms of lost profits nearly always exceeds the benefit from any reduction in pollution from thermal fired generation. Only for the most restrictive ramping rate (250 CFS-hr) and with the higher estimate for marginal external costs of pollution do we observe that the benefit from reduced thermal emissions exceeds the cost from lost profit. If we could measure the associated environmental benefits for the river ecosystem, these could be directly included in a cost benefit analysis. The net cost lines show how large this benefit would have to be to justify ramping restrictions. Using the high marginal external cost estimate (green lines) we see that the necessary ecosystem benefit actually declines as ramping constraints are made more restrictive, getting smaller from 1,000 CFS-hr.

\footnotetext{
${ }^{24}$ These estimates are first calculated based on the coal generation plant's marginal external costs (MEC) in the US in 2004 (Dewees [2008]). We use the Michigan MEC at $34.77 \$$ US/MWh (low MEC scenario), and the Ohio MEC at 51.68 WUS/MWh (high MEC scenario). Then these values are converted to Canadian dollars at the 2004 exchange rate of $1.3 \$ C A D / \$ U S$. Natural gas emissions and external costs for gas-fired power plants are much lower than those of coal. The gas-fired power plant's marginal external cost is 5.72 \$US/MWh for Michigan (low MEC scenario) and 7.66 \$US/MWh for Indiana (high MEC scenario).

${ }^{25}$ 'Benefit' curves show the extra environmental benefit under various levels of ramping rate restrictions using either $67.18 \$ / \mathrm{MWh}$ or $45.20 \$ / \mathrm{MWh}$ as a proxy for the marginal environmental cost of coal fired power. 'Cost' refers to the generator's cost (reduction of profit) under various levels of ramping rate restrictions. The 'net cost' curves show the 'cost' minus 'benefit' for the two different estimate of the marginal environmental cost of thermal power.
} 


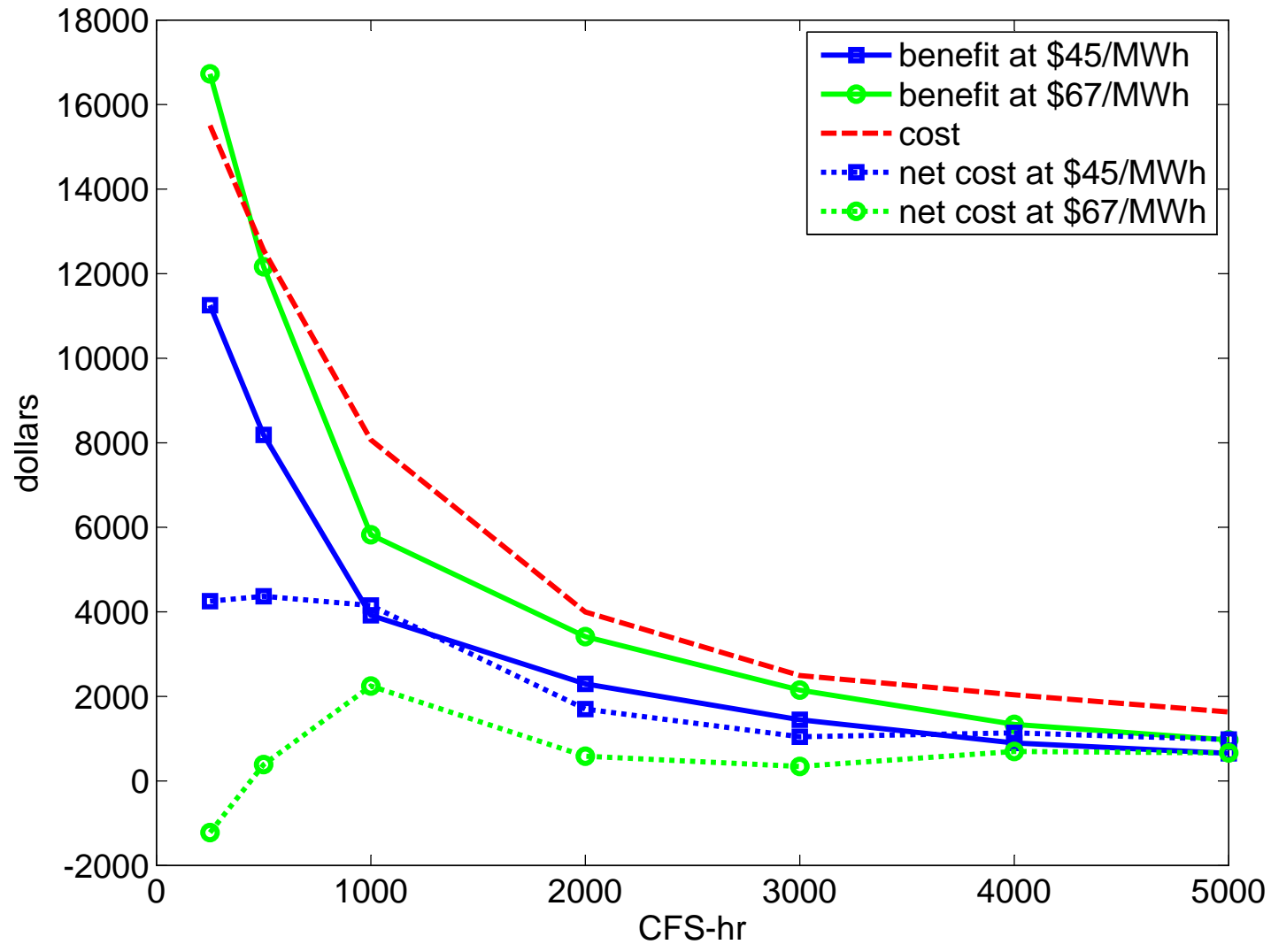

Figure 2.8: Net Cost Analysis under Various Equal Levels of Up-ramping and Downramping Constraints (Coal as Replacement Power) 


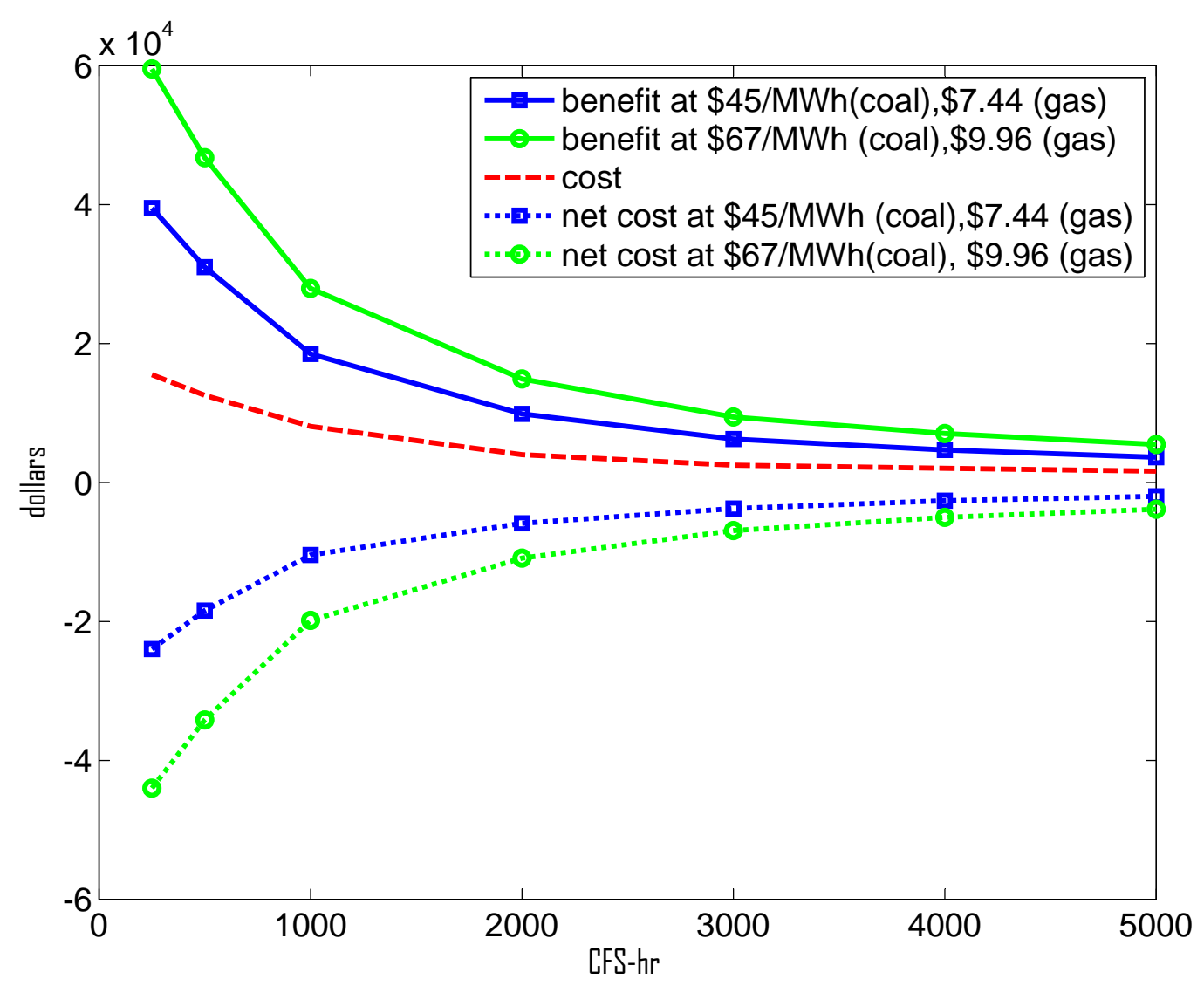

Figure 2.9: Net Cost Analysis under Various Equal Levels of Up-ramping and Downramping Constraints (Coal and Natural Gas as Replacement Power)

The results for the scenario with coal as replacement power during the off-peak period and natural gas as replacement power during the on-peak period under various equal levels of up-ramping and down-ramping constraints are reported in Figure $2.9^{26}$. With more and

\footnotetext{
${ }^{26}$ 'Benefit' curves show the extra environmental benefit under various levels of ramping rate restrictions using either $67.18 \$ / \mathrm{MWh}$ or $45.20 \$ / \mathrm{MWh}$ as a proxy for the marginal environmental cost of coal fired power and $9.96 \$ / \mathrm{MWh}$ or $7.44 \$ / \mathrm{MWh}$ as the marginal environmental cost of natural gas fired power. 'Cost' refers to the generator's cost (reduction of profit) under various levels of ramping rate restrictions. The 'net cost' curves show the 'cost' minus 'benefit' for the two different estimate of the marginal environmental cost of thermal power.
} 
more restrictive ramping constraints, both the cost curve and the environmental benefit curves move up steadily, but the environmental benefit curves increase at a slightly faster rate and are always located above the cost curve. The net cost curves are always below zero and move down steadily with increasing ramping restrictions. This follows because with increasing ramping restrictions the environmental benefit gained through the reduction of thermal power generated using coal in the off-peak period exceeds by an increasing amount the associated loss of profit and the environmental cost of increased thermal power generated using natural gas in the on-peak period. In this example, the greater the ramping restrictions, the greater the net social benefit. Optimal ramping restrictions are shown to be 250 CFS-hr even without any consideration of the potential benefits to the river ecosystem.

Our assumption of a one-for-one replacement of thermal power by hydro power, with no effects on price, is clearly overly simplistic, but illustrates the importance of looking at the impact of hydro ramping rates on other sources of electricity generation. In Ontario, coal generation is being phased out as part of government policy to reduce air pollution. As the province moves to "greener" sources of power the potential for an associated positive impact of ramping restrictions on air quality will be reduced.

\subsection{Conclusions}

The ability of hydro facilities to respond quickly through ramping to changing demand conditions is one of the benefits of hydro power. However the possibility of negative consequences of ramping on aquatic ecosystems needs to be considered by regulators. These negative impacts are case specific, dependent on the ecological conditions of particular rivers and streams. In cases where ramping rate restrictions are being considered, there should be a recognition of the costs imposed on hydro operators in terms of lost profits as well as potential environmental impacts that result from the need to utilize alternative sources of electricity. Ideally ramping rate regulations would be determined through a careful analysis of all the potential impacts. This chapter contributes to our understanding of these impacts and the trade offs involved. 
For a prototype hydro dam we modelled the lost profits for a range of ramping restrictions over a five day period. We present results for a typical day once a steady state has been obtained. We find that profits are significantly affected (by about $8 \%$ ) in the case of the most severe ramping constraints. However we also find a range of less severe ramping constraints for which profits are impacted by less than $3 \%$. We examine the change in total hydro production, as well as the purchase of replacement power that results from the restrictions. One counter intuitive result is that total hydro production increases as a result of the ramping constraints. This result follows from the desire of the hydro operator to mitigate the effect of the ramping constraints by producing more power in off-peak periods and in our example resulted in an increase in the average water level in the dam over a 24 hour period. Our assumption is that the increase in hydro production will result in reduced thermal generation in the economy, which causes an environmental benefit from reduced air pollution emissions. We calculate a net cost of the ramping restrictions as the lost profits net of any environmental benefit of reduced air pollution. This net cost can be compared to expected environmental benefits from an improved aquatic ecosystem.

An important conclusion of the chapter is that ramping restrictions should not be determined in isolation, but rather using a cost-benefit approach that evaluates the trade offs involved. This chapter has identified some of the important trade offs that should be examined more carefully in future research. These include the impact on hydro operator profits as well as the environmental impact of a change in the intensity of use of other types of power.

There are several directions for further research. First, we could account for uncertainty in demand, water inflow and electricity prices through a stochastic dynamic optimization model assuming these uncertain variables can be modelled as known stochastic processes. Second, more realistic, but sophisticated hydro power production functions could be used and the provision of ancillary services such as spinning reserve to the electricity market could be considered. Finally further efforts are needed to construct a measure of the environmental benefits for the river ecosystem gained by imposing these ramping restrictions. 


\section{Chapter 3}

\section{An Options Pricing Approach to Ramping Rate Restrictions at Hydro Power Plants}

\subsection{Introduction}

Electricity demand tends to follow a marked daily pattern, peaking during the daytime and early evening hours. It is also subject to seasonal spikes to meet demand for air conditioning in the summer and/or heating in the winter. Electricity cannot be stored and the typical base load power sources including coal and nuclear are much more limited than hydro in their ability to vary generation levels. This results in fairly inelastic supply and consequent volatile electricity prices, particularly in those regions which rely heavily on fossil fuel and nuclear power. Price spikes and jumps are not uncommon. Figure 3.1 shows the hourly German EEX spot price from January 1 to July 24, 2006. Hydro operators can benefit from this volatility by increasing water release rates (ramping up) in response to high prices and by reducing water release rates (ramping down) in periods of low prices to let water levels recover in the reservoir. 


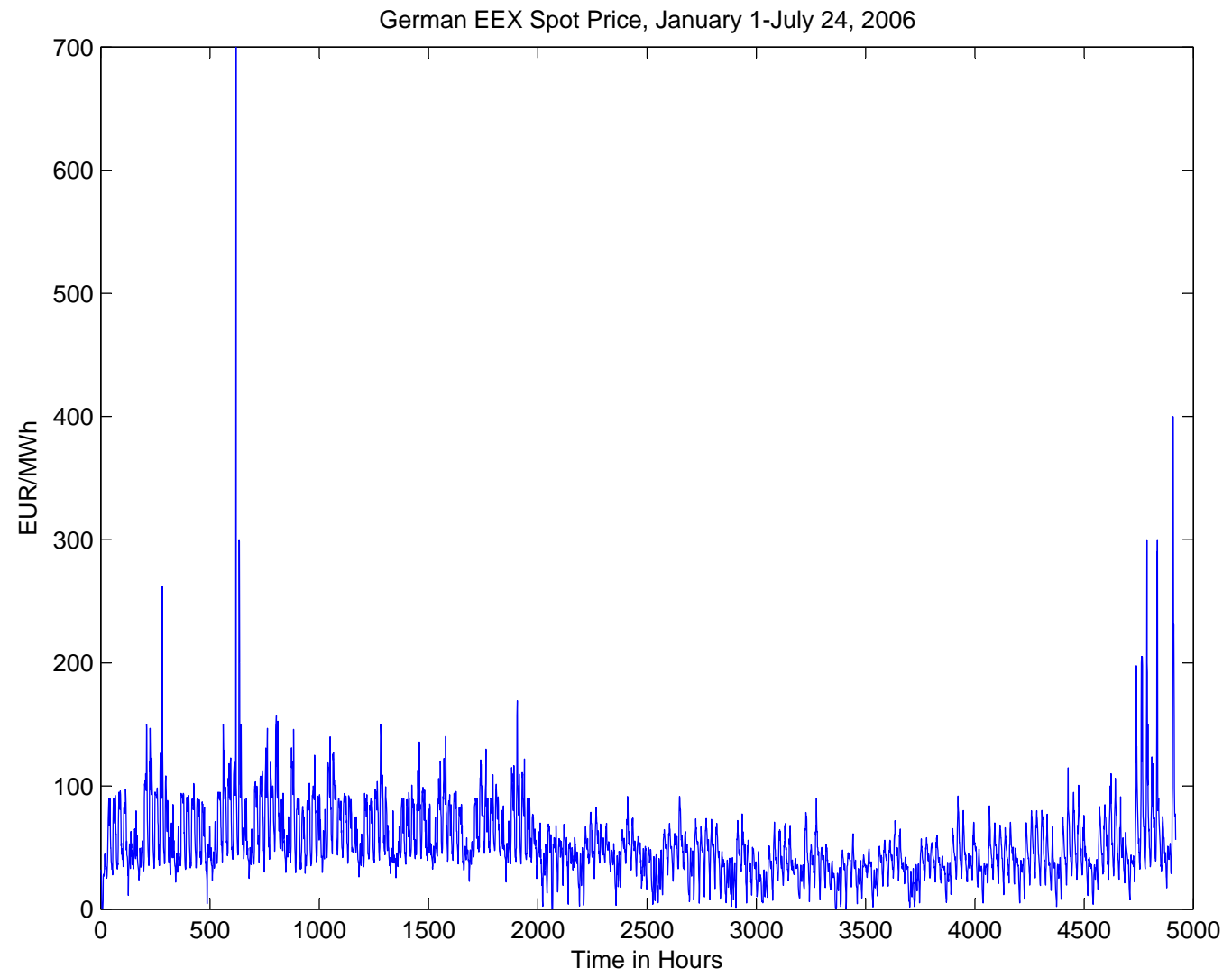

Figure 3.1: German EEX Spot Price, January 1-July 24, 2006

Regulators charged with protecting local ecosystems must consider the consequences of hydro operations for native flora and fish habitat. Restrictions may be imposed on minimum and maximum water levels in reservoirs and rivers, release rates from reservoirs, and the rate of change in the release rate (ramping rate). Any restrictions on hydro operations must be considered in light of the impact on profitability for the hydro operator and on the capability of the electricity grid to meet peak demands. If there is greater reliance on fossil fuels for peaking requirements, there would be added environmental consequences resulting from the harmful emissions from coal, natural gas and petroleum ${ }^{1}$. The optimal

\footnotetext{
${ }^{1}$ This issue is addressed in chapter 2 in a model of optimal hydro production with deterministic prices.
} 
choice of restrictions would balance the consequences for the ecosystem with the lost profits to hydro operators and the possible impact on the electricity grid.

Intuitively, ramping restrictions should have the largest impact on profits of hydro operations for which the optimal control without restrictions involves frequent ramping up or down of water release rates. This would be expected in an environment of frequently changing prices in which hydro operators are motivated to adjust water release rates accordingly. It follows that in analyzing the impact of ramping rates it is important to pay particular attention to accurately modelling the dynamics of electricity prices. The jumps and spikes that are observed in electricity prices make electricity price modelling particularly challenging. The recent literature on electricity price modelling, reviewed in Section 3.2 , suggests that regime switching models hold considerable promise.

In the current literature, there are relatively few studies of the costs and associated benefits of restricting ramping rates at hydro power plants. This chapter contributes to this literature by studying the impact of ramping restrictions on hydro plant operations and profitability using a regime switching model of electricity prices, which we argue provides a more realistic characterization of electricity prices than other models, such as jump diffusion, used previously in the literature. Hydro operations are modelled as a stochastic control problem which results in a Hamilton-Jacobi-Bellman (HJB) equation. The ramping rate for water releases is chosen to maximize the value of the hydro plant. The optimal control is determined by solving the HJB equation numerically using a fully implicit finite difference approach with semi-Lagrangian time stepping. ${ }^{2}$ We examine the impact of a range of ramping restrictions for a medium-sized prototype hydro plant in Ontario. We focus on how the impact of ramping restrictions depends on the cost of generating hydroelectric power and the characteristics of the assumed price process such as the volatility, probability of regime shift, speed of mean reversion and long run average price. For comparison we also examine results for a single price regime case (using the base regime in the regime switching model as the single regime) to determine how the presence of two price

\footnotetext{
${ }^{2}$ See Chen [2008] for a discussion of finite difference schemes based on a semi-Lagrangian method for solving stochastic optimal control problems.
} 
regimes affects hydro plant value and optimal operations. The parameters ${ }^{3}$ of the regime switching model are based on estimates from Janczura and Weron [2009].

Our study is limited in that we do not address the environmental gains to the aquatic ecosystem, nor the environmental costs of alternate thermal power generation. Specifying these environmental costs and benefits in monetary terms is problematic and beyond the scope of this research. Rather we seek to examine the other side of the equation - the costs of these restrictions in terms of lost profitability. Knowledge of these costs will help illuminate the trade offs involved and inform the design of regulations.

Individual hydro plants are unique in terms of their size, water flows, physical design and linkages to the grid. Optimal hydro operations depend on electricity market structure, demand patterns and reliance on different fuel sources. In this chapter we seek to draw some general conclusions about when the effect of ramping restrictions on hydro profits will be most severe. To preview our results, for our medium-sized power plant there is a significant effect on profits for the most restrictive ramping constraints, but we also observe a range of ramping restrictions over which profits are not substantially affected. There are differing effects depending on the characteristics of the assumed price process. We find that ramping restrictions have a larger impact when the expected variation in price is increased such as through an increase in volatility which make it desirable to change water release rates relatively frequently. Ramping rates also have a larger impact if the range of likely prices includes values at which the hydro plant is operating at a loss. These and other avenues through which ramping restrictions influence profits are explored in depth in this chapter.

This chapter is organized as follows: in the second section, we provide a brief review of the related literature; electricity price models for both the jump diffusion and regime switching cases are presented in section three; section four describes the modelling of hydro operations; in section five the Hamilton Jacobi Bellman equation for the regime switching case is derived; section six describes the prototype hydro plant used in the empirical study as well as the parameters of the price process; section seven contains the empirical analysis

\footnotetext{
${ }^{3}$ We use the estimated parameters by Janczura and Weron [2009] based on the German EEX spot price since these parameters for the Ontario spot price are not available in the literature.
} 
of the hydro operation and profit under various ramping restrictions, together with a comprehensive sensitivity analysis; lastly, conclusions and directions of future research are given in section eight.

\subsection{Related Literature}

Early work on the economics of ramping restrictions include Veselka et al. [1995], Edwards et al. [1999], Harpman [1999] and Edwards [2003], who examine the effect of particular ramping rate regimes as environmental constraints, but do not provide extensive analysis of ramping rate restrictions on the power station's optimal operation and profit. The trade offs involved in the choice of the optimal ramping rate regime are not addressed in these papers. In a recent study, Niu and Insley [2013] extend these works by considering both the associated benefits and costs of ramping restrictions on hydro profits and on total daily hydro production and the potential implications for other sources of power.

A limitation of these studies is that the optimization models are solved in a deterministic framework. Uncertain electricity prices as well as water inflows imply that optimal hydro power operation is best studied in a stochastic framework. Thompson et al. [2004] and Chen and Forsyth [2008] study the hydro operation and valuation problem in a stochastic optimal control framework using jump diffusion models for electricity prices. Chen and Forsyth [2008] also analyze the impact of ramping restrictions on a hydro plant's value. In a numerical example they find that imposing the most restrictive ramping constraint reduces the value of a hypothetical power plant by $37 \%$. The focus of the Chen and Forsyth [2008] paper is on the efficient solution of the complex stochastic optimization problem. They do not attempt to determine the best model for electricity prices.

There is a rich literature which addresses the challenges of modelling electricity spot prices. At the heart of the modelling issues are the inherent properties of electricity which result in limited transferability over time (storage constraint) and space (transmission constraint). Key characteristics of electricity prices and loads include strong dependence on weather and regular daily, weekly, and monthly patterns. Electricity spot prices tend 
to exhibit frequent small jumps and occasionally extreme spikes. They show strong mean reversion moving rapidly from price spikes to the mean price, and price level and volatility are highly correlated. Weron [2008] gives a description of these stylized facts.

Weron [2008] provides a good review of the literature on electricity price modelling. When the objective is derivative pricing and risk management, there are two popular classes of stochastic processes for electricity prices: mean-reverting jump diffusion models and mean-reverting Markov regime switching models ${ }^{4}$. Some examples of jump-diffusion models include Deng [2000], Geman and Roncoroni [2006], Escribano et al. [2011], Weron [2008] and Benth et al. [2007]. An issue in using jump diffusion models is how to capture the return to a more normal price level after a jump. There are various modelling approaches used in the literature. Chen and Forsyth [2008] in their numerical example model electricity prices with two jump processes - a positive jump is followed with high probability by a down jump, bring price back into its normal state. Weron et al. [2004] suggest this represents a good approximation as spikes typically do not last for more than one day.

It is, however, desirable to be able to capture the situation where price suddenly spikes upward and then remains in this spike regime for several periods before returning to a more normal state. This can be observed in the historical data as is shown in Figure 3.2. This pattern might appear when for some reason the grid cannot respond quickly enough to a sudden surge in demand or when there is an unanticipated restriction on supply such as would be caused by grid congestion or an outage in a power station. One way to capture this phenomenon would be to add more jump terms into a jump diffusion model, but this would make the parameter estimation and plant valuation quite complex. Another alternative is the use of Markov regime switching models with base and jump regimes where there is some positive probability of remaining in the jump regime.

\footnotetext{
${ }^{4}$ Another class of model is the threshold autoregressive (TAR) model for electricity prices. Recent work includes Rambharat et al. [2005] who incorporate an exogenous variable (temperature) in the proposed TAR model. However, Misiorek et al. [2006] argue that the spot electricity price depends on both fundamentals (such as loads and network constraints) and other psychological and sociological factors which are unquantifiable. They state that "the Markov regime-switching (or simply regime-switching) models, where the regime is determined by an unobservable, latent variable, seem interesting."
} 


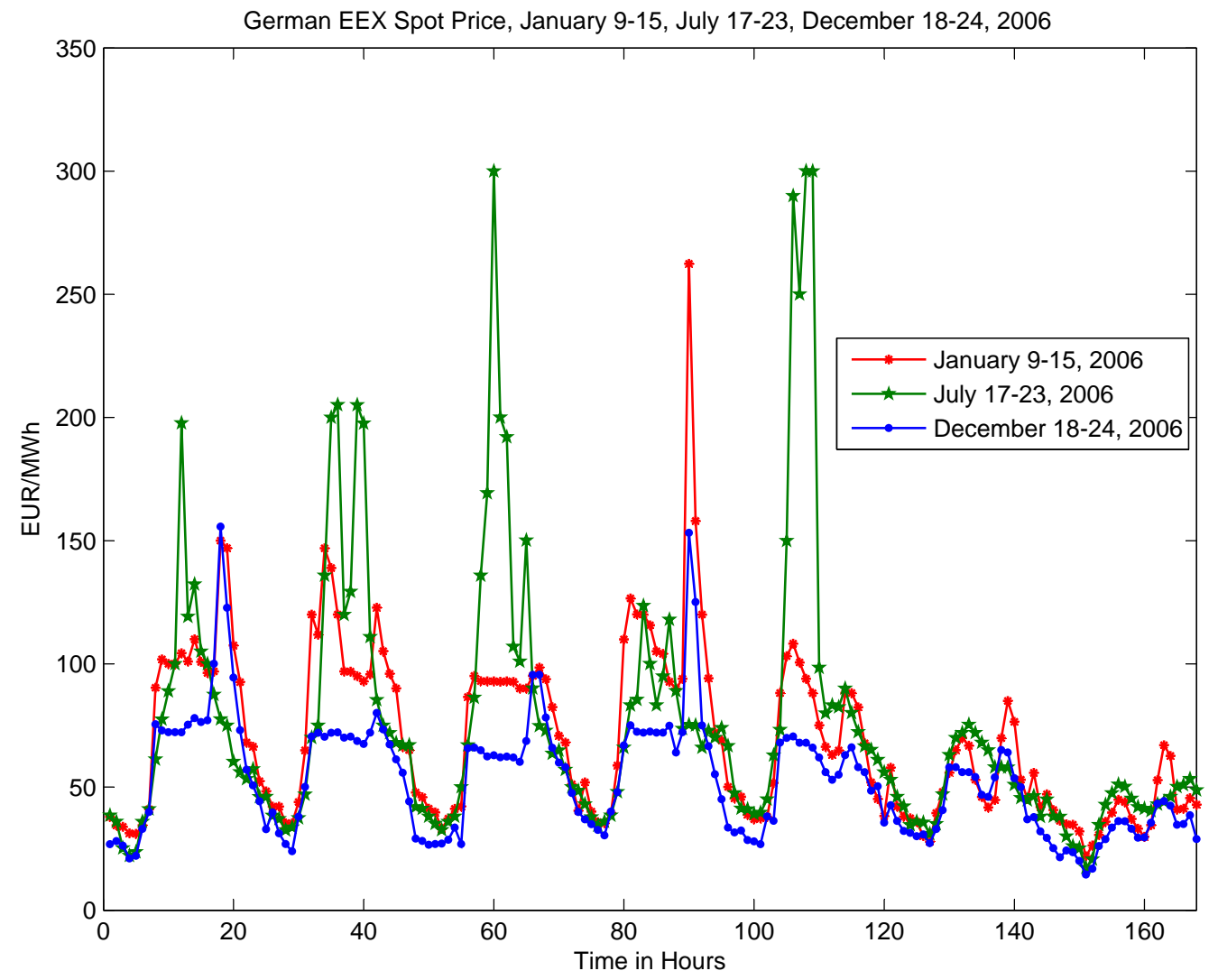

Figure 3.2: German EEX Spot Price, January 9-15, July 17-23, December 18-24, 2006

Early results on Markov regime switching models include Ethier and Mount [1998] who estimate a two-state switching model with both regimes governed by $\mathrm{AR}(1)$ processes. Huisman and De Jong [2003], Weron et al. [2004] and Bierbrauer et al. [2007] make extensions by proposing an independent spike two-regime model using different distributional assumptions for the spike regime. De Jong [2006] modifies the spike regime by using an autoregressive process with Poisson jumps. Further extensions are given by Mount et al. [2006] who allow transition probabilities to depend on the reserve margin and Huisman [2008] who suggests temperature dependent transition probabilities. In two recent studies, Janczura and Weron [2009, 2010] introduce median-shifted spike regime distributions 
with heteroskedastic dynamics for the base regime. An overview of the development on alternative regime switching models can be found in Janczura and Weron [2010]. Some applications of regime switching models in the valuation of physical assets include Chen and Forsyth [2010] for natural gas storage valuation, Heydari and Siddiqui [2010] for valuing a gas-fired power plant and Chen and Insley [2012] for optimal forest harvesting problem.

One common practice with the study of electricity price dynamics is that daily average log-prices, instead of hourly prices, are used to estimate the chosen models partially because they are better suited to certain distributions and the estimated parameters have robust statistical properties. However, this may be problematic for derivative pricing and risk management since optimal decisions such as power plant operations are made on an hourly or half hourly basis according to spot electricity prices and demand.

\subsection{Electricity Price Models}

Both jump diffusion and regime switching models have been used in the literature to model electricity prices. Chen and Forsyth [2008] and Thompson et al. [2004] employ jump diffusion models as shown in Equation (3.1).

$$
d P=\mu(P, t) d t+\sigma(P, t) d W+\sum_{\varsigma=1}^{N} \psi_{\varsigma}\left(P, t, J_{\varsigma}\right) d q_{\varsigma} .
$$

where

- $\mu(P, t)$ is the generalized drift;

- $\sigma(P, t)$ is the generalized volatility;

- $d W$ is the increment of the standard Gauss-Wiener process;

- $\psi_{\varsigma}\left(P, t, J_{\varsigma}\right)$ 's are any arbitrary functions of price and/or time;

- $J_{\varsigma}$ 's are drawn from some arbitrary distributions $G_{\varsigma}(J)$; 
- $d q_{\varsigma}$ 's are Poisson processes with

$$
d q_{\varsigma}= \begin{cases}1 & \text { with probability } \epsilon_{\varsigma}(P, t) d t \\ 0 & \text { with probability } 1-\epsilon_{\varsigma}(P, t) d t\end{cases}
$$

Over the time interval $d t, \epsilon_{\varsigma}(P, t) d t$ is the probability of $d q_{\varsigma}=1$, i.e., the price jumps from $P$ to $P+\psi_{\varsigma}\left(P, t, J_{\varsigma}\right)$. Here $\psi_{\varsigma}\left(P, t, J_{\varsigma}\right)$ determines the jump size of a particular jump, where $J$ is a random variable. The number of Poisson processes $N$ is arbitrary. In both Chen and Forsyth [2008] and Thompson et al. [2004] there are assumed to be two types of jumps, an upward jump and a downward jump. A threshold, $\bar{P}$, was specified so that any price above the threshold was assumed to have arrived there as a result of an up-jump. A seasonal component is included in the drift term. Parameters are chosen by Thompson et al. [2004] so that resulting price simulations appear to be a reasonable depiction of electricity prices. Chen and Forsyth [2008] adopt the same parameter values. Note that the parameter values are assumed to reflect risk adjusted parameters under the Q measure.

The regime switching price model defines $N$ specific regimes and a different price process may be specified for each regime. The transition between regimes is controlled by a continuous-time Markov chain. A general specification of a regime switching process is given in Equation (3.2) below.

$$
d P=\mu^{\imath}(P, t) d t+\sigma^{\imath}(P, t) d Z+\sum_{\jmath=1}^{N} P\left(\xi^{\imath \jmath}-1\right) d X_{\imath \jmath}, \imath=1, \ldots, N .
$$

where

- $\mu^{\imath}(P, t)$ is the generalized drift in state $\imath$;

- $\sigma^{\imath}(P, t)$ is the generalized volatility in state $\imath$;

- $d Z$ is the increment of the standard Gauss-Wiener process;

- $\xi^{\imath \jmath}$ is the jump size from state $\imath$ to state $\jmath$; 
- $d X_{\imath \jmath}$ indicates the transition of the Markov chain from state $\imath$ to state $\jmath$;

Let $q_{\imath}$ be the transition intensity of the Markov chain from state $\imath$ to state $\jmath$. Then over an instant $d t$, we have

$$
d X_{\imath \jmath}= \begin{cases}1 & \text { with probability } q_{\imath \jmath} d t \\ 0 & \text { with probability } 1-q_{\imath \jmath} d t\end{cases}
$$

Over the time interval $d t, q_{\imath \jmath} d t$ is the probability of $d X_{\imath \jmath}=1$, i.e., the price jumps from $P$ in regime $\imath$ to $P \xi^{\imath \jmath}$ in regime $\jmath$. The number of states $N$ is often chosen arbitrarily for ease of computation, and is set equal to 2 in our empirical study.

Note that in the regime switching model the drift and diffusion terms are specific to the particular regime, indexed by superscript $\imath$. When a regime change occurs, the price jumps from $P$ in regime $\imath$ to $P \xi^{\imath \jmath}$ in regime $\jmath$ and both the drift function and volatility function will change from $\mu^{\imath}(P, t)$ and $\sigma^{\imath}(P, t)$ to $\mu^{\jmath}(P, t)$ and $\sigma^{\jmath}(P, t)$. This contrasts with the common jump diffusion model in which a jump in price does not imply a change in drift and volatility terms. The regime switching model could approximate a jump diffusion

model if many different regimes are assumed, and each regime involves a different jump size.

There is no general agreement in the literature as to which model is best able to describe the characteristics of electricity prices. This is an empirical question and the answer will be case specific, depending on the nature of a given electricity market. In this chapter we adopt a regime switching model based on a statistical estimation done by Janczura and Weron [2009] for German EEX spot prices.

\subsection{Modelling Optimal Hydro Operations}

\subsubsection{Physical and Environmental Constraints}

A typical hydro power plant is operated under certain physical and environmental constraints including minimum and maximum water release rates, maximum and minimum 
water content in the reservoir, and possibly ramping constraints. We specify similar constraints as in Niu and Insley [2013]. The minimum and maximum water release rate requirements can be represented as

$$
r^{\min } \leq r \leq r^{\max }
$$

where $r$ is the water release rate. Reservoir storage constraints can be stated as

$$
w^{\min } \leq w \leq w^{\max }
$$

where $w$ represents the water content. The equation of motion for water is governed by the following formula:

$$
d w=a(\ell-r) d t
$$

where $a$ converts water flow rate to the same unit as the water content. Water inflow rates are stochastic in nature. In order to focus on the stochastic feature of electricity prices and the impact of ramping restrictions on the valuation and optimal operation of the hydro power plant, we assume that the inflow rate $\ell$ is a positive constant. This simplified assumption is realistic for a short period of time such as the single week used in the empirical analysis in Section 3.6. An extension of this chapter would be to model $\ell$ by a stochastic differential equation. To prevent the water content falling below $w^{\text {min }}$ or increasing above $w^{\max }$, we follow the approach of Chen and Forsyth [2008] using an augmented equation of motion:

$$
d w=H(r, w) a(\ell-r) d t
$$

for any $w \in\left[w^{\min }, w^{\max }\right]$ and $r \in\left[r^{\min }, r^{\max }\right]$, where $H$ is a smooth function of $w$ and $r$ satisfying

$$
\begin{aligned}
& H(r, w) \rightarrow 0 \text { if } r>\ell \text { and } w \rightarrow w^{\min }, \\
& H(r, w) \rightarrow 0 \text { if } r<\ell \text { and } w \rightarrow w^{\max }, \\
& H(r, w)=1 \text { otherwise. }
\end{aligned}
$$


In order to satisfy Equation (3.7) it is assumed that function $H(r, w)$ has the following properties:

$$
\begin{aligned}
& H(r, w)=O\left(\left(w-w^{\min }\right)^{\nu}\right) \text { if } r>\ell \text { and } w \rightarrow w^{\min }, \\
& H(r, w)=O\left(\left(w^{\max }-w\right)^{\nu}\right) \text { if } r<\ell \text { and } w \rightarrow w^{\max }, \\
& H(r, w)=1 \text { otherwise }
\end{aligned}
$$

where $\nu$ could take any small positive constant value.

The ramping control variable $z$ is defined in the following equation:

$$
d r=z d t
$$

The ramping rate $z$ needs to satisfy the following conditions

$$
\begin{aligned}
& z \geq 0 \text { if } r=r^{\min } . \\
& z \leq 0 \text { if } r=r^{\max } .
\end{aligned}
$$

to avoid the water release rate from increasing above the maximum releasing rate or decreasing below the minimum releasing rate. As in Chen and Forsyth [2008], in order to satisfy conditions in $(3.10)$ we will require that

$$
\begin{aligned}
& \min \{z\}=O\left(\left(r-r^{\min }\right)^{\theta}\right) \text { if } r \rightarrow r^{\min }, \\
& \max \{z\}=O\left(\left(r^{\max }-r\right)^{\theta}\right) \text { if } r \rightarrow r^{\max },
\end{aligned}
$$

where $\theta$ could take any small positive constant value. In addition, the up-ramping and down-ramping constraints can be expressed as

$$
\begin{gathered}
d r \leq r^{u} d t . \\
-d r \leq r^{d} d t .
\end{gathered}
$$


where $r^{u}$ and $r^{d}$ represent the maximum allowed up-ramping and down-ramping rates respectively. We rewrite these two equations as

$$
-r^{d} d t \leq d r \leq r^{u} d t
$$

which is

$$
-r^{d} \leq z \leq r^{u}
$$

Let $Z(r)$ denote the set of admissible controls that satisfy constraint (3.15) and conditions (3.11). This gives $Z(r) \subseteq\left[z^{\min }, z^{\max }\right]$.

\subsubsection{The Optimization Problem}

The present value of net revenue from power generation from $t=t_{1}$ to $t=T$ is given by the following equation

$$
\int_{t_{1}}^{T} e^{-\rho t} q(r, h(w))(P-c) d t
$$

where $\rho$ is the discount rate, $q$ is the amount of power produced which is a function of the water release rate $r$ and the head $h$, and $c$ is the unit cost of hydro power production, which is assumed to be a positive constant. Therefore, $q(r, h(w))(P-c)$ is the instantaneous profit for the hydro power plant. The objective is to maximize Equation (3.16) subject to the set of physical and environmental constraints for a hydro power plant described in Section 3.4.1. Let $V^{\imath}\left(P, w, r, t_{1}\right)$ denote the value of the hydro plant under the optimal control in regime $\imath$ with the risk adjusted (or risk neutral) measure.

$$
V^{\imath}\left(P, w, r, t_{1}\right)=\max _{z} E^{Q}\left[\int_{t_{1}}^{T} e^{-\rho\left(t-t_{1}\right)} H(r, w) q(r, h(w))(P-c) d t\right]
$$

subject to

$$
Z(r) \subseteq\left[z^{\min }, z^{\max }\right]
$$




$$
\begin{gathered}
d w=H(r, w) a(\ell-r) d t . \\
d r=z d t . \\
d P=\mu^{\imath}(P, t) d t+\sigma^{\imath}(P, t) d Z+\sum_{\jmath=1}^{N} P\left(\xi^{\imath \jmath}-1\right) d X_{\imath \jmath} .
\end{gathered}
$$

where $H$ is a penalty function on profits given by Equation (3.7), so that the profit decreases to zero as $r>\ell$ and $w \rightarrow w^{\min }$ or $r<\ell$ and $w \rightarrow w^{\max }$, and otherwise remains at $H(r, w) q(r, h(w))(P-c)$. $Z(r)$ specifies the set of admissible controls. In addition, the electricity price, $P$, follows Equation (3.21) which is assumed to be a risk adjusted specification. The transition equations governing water balance and water release are specified in Equations (3.19) and (3.20) respectively. Note that constraints (3.3) and (3.14) are incorporated into (3.18) and constraint (3.4) is incorporated into (3.17) and (3.19).

\subsection{Hamilton Jacobi Bellman Equations}

\subsubsection{HJB-PDE for the Regime Switching Model}

Following Kennedy [2007], we use the standard hedging approach in options pricing to derive the HJB-PDE (Hamilton Jacobi Bellman Partial Differential Equation) for the value of the hydro power station (Appendix A.1.1). Under the risk adjusted measure ${ }^{5}$ the value of the hydro plant in regime $\imath$ satisfies the following equation

\footnotetext{
${ }^{5}$ The hydro plant valuation with the regime switching model for electricity prices is associated with the regime switching risk, therefore the market is not complete. This indicates that there is no unique equivalent martingale measure that allows us to move from the physical measure to the risk adjusted measure. Forsyth and Vetzal [2014] discuss that one way to pin down the measure for pricing is to use an expanded set of hedging instruments. Then the risk-neutral transition intensity could be uniquely determined by the prices of these instruments. Our derivation for the HJB-PDE follows this method.
} 


$$
\begin{array}{r}
\bar{r} V^{\imath}=\sup _{z \in Z(r)}\left(z \frac{\partial V^{\imath}}{\partial r}\right)+H(r, w) a(\ell-r) \frac{\partial V^{\imath}}{\partial w}+\frac{1}{2}\left(\sigma^{\imath}\right)^{2}(P, t) \frac{\partial^{2} V^{\imath}}{\partial P^{2}}+\left(\mu^{\imath}(P, t)-\Lambda^{\imath} \sigma^{\imath}(P, t)\right) \frac{\partial V^{\imath}}{\partial P} \\
+H(r, w) q(r, h(w))(P-c)+\frac{\partial V^{\imath}}{\partial t}+\sum_{\substack{\jmath=1 \\
\jmath \neq \imath}}^{N} \lambda_{\imath \jmath}^{Q}\left(V^{\jmath}-V^{\imath}\right) .
\end{array}
$$

where $\bar{r}$ is the risk free interest rate, $\Lambda^{\imath}$ is the market price of risk in state $\imath$ and $\lambda_{\imath \jmath}^{Q}$ is the risk-neutral transition intensity from state $\imath$ to $\jmath(\jmath \neq \imath)$. The electricity price given by Equation (3.2) is used to derive Equation (3.22).

The HJB-PDE can be interpreted as a no-arbitrage condition. In the risk neutral world the return on the asset must equal the risk free rate, $\bar{r}$. On the right hand side of the equation we see all the sources of return on the asset which, from left to right, include changes due to the optimal choice of the ramping rate, $z$; changes due to water inflows and releases which affect the water content, $w$; changes due to first and second order effects of electricity price variation, $P$; cash flow from electricity sales; changes in $V$ with time; and lastly the effect on value of the risk of a price regime change.

The domain for Equation $(3.22)$ is $(P, w, r) \in[0, \infty] \times\left[w^{\min }, w^{\max }\right] \times\left[r^{\min }, r^{\max }\right]$. The numerical solution is computed in a finite domain: $(P, w, r) \in\left[0, P^{\max }\right] \times\left[w^{\min }, w^{\max }\right] \times$ $\left[r^{\min }, r^{\max }\right]$. Equation (3.22) is solved backwards in time from $t=T$ to $t=t_{1}$. It is convenient to define $\tau=T-t$ as the time remaining in the life of the asset. Equation (3.22) can be rewritten in terms of $\tau$ as follows:

$$
\frac{\partial V^{\imath}}{\partial \tau}=C V^{\imath}+B V^{\imath}+\sup _{z \in Z(r)}\left(z \frac{\partial V^{\imath}}{\partial r}\right)+H(r, w) a(\ell-r) \frac{\partial V^{\imath}}{\partial w}+H(r, w) q(r, h(w))(P-c) .
$$

where the operators $C$ and $B$ are given by 


$$
\begin{aligned}
C V^{\imath} & =\frac{1}{2}\left(\sigma^{\imath}\right)^{2}(P, t) \frac{\partial^{2} V^{\imath}}{\partial P^{2}}+\left(\mu^{\imath}(P, t)-\Lambda^{\imath} \sigma^{\imath}(P, t)\right) \frac{\partial V^{\imath}}{\partial P}-\left(\bar{r}+\sum_{\substack{\jmath=1 \\
\jmath \neq \imath}}^{N} \lambda_{\imath \jmath}^{Q}\right) V^{\imath} . \\
B V^{\imath} & =\sum_{\substack{\jmath=1 \\
\jmath \neq \imath}}^{N} \lambda_{\imath \jmath}^{Q} V^{\jmath} .
\end{aligned}
$$

We specify a functional form of the electricity price as in Chen and Forsyth [2008] for both the drift and diffusion terms and assume $\mu^{\imath}(P, t)=\alpha^{\imath}\left(K^{\imath}-P\right), \sigma^{\imath}(P, t)=\sigma^{\imath} P$. Now $C V^{\imath}$ is written as

$$
C V^{\imath}=\frac{1}{2}\left(\sigma^{\imath}\right)^{2} P^{2} \frac{\partial^{2} V^{\imath}}{\partial P^{2}}+\left[\alpha^{\imath}\left(K^{\imath}-P\right)-\Lambda^{\imath} \sigma^{\imath} P\right] \frac{\partial V^{\imath}}{\partial P}-\left(\bar{r}+\sum_{\substack{\jmath=1 \\ j \neq \imath}}^{N} \lambda_{\imath \jmath}^{Q}\right) V^{\imath} .
$$

A numerical approach is required to solve this non-linear HJB-PDE. We will use a fully implicit finite difference scheme with semi-Lagrangian time stepping. This numerical approach converges to the viscosity solution of the HJB equation as is shown in Chen and Forsyth [2007, 2008]. Details on the numerical algorithms for solving Equation (3.23) are given in A.1.2.

\subsubsection{Boundary Conditions}

Boundary conditions are required to fully specify the optimization problem. For the terminal boundary condition at $t=T$ (i.e. $\tau=0$ ), we assume the value of the asset is zero, which is a common assumption in the literature. (See Thompson et al. [2004] for example.)

$$
V^{\imath}(P, w, r, \tau=0)=0 \text {. }
$$

It can be shown that the solution of Equation (3.23) does not require information outside of the domains of $w$ and $r$ and hence no special boundary conditions are required. 
At $w^{\min }, w^{\max }, r^{\min }, r^{\max }$, we simply solve the PDE along the corresponding boundaries. ${ }^{6}$ However, the computational domain of the price could differ for any two different regimes.

Taking the limit of the PDE equation as $P \rightarrow 0$, we obtain the boundary PDE for $\imath=1,2, \ldots, N$.

$$
\frac{\partial V^{\imath}}{\partial \tau}=C_{0} V^{\imath}+B V^{\imath}+\sup _{z \in Z(r)}\left(z \frac{\partial V^{\imath}}{\partial r}\right)+H(r, w) a(\ell-r) \frac{\partial V^{\imath}}{\partial w}-H(r, w) q(r, h(w)) c ; P \rightarrow 0
$$

where the operator $B$ is the same as above and $C_{0}$ is given by

$$
C_{0} V^{\imath}=\alpha^{\imath} K^{\imath} \frac{\partial V^{\imath}}{\partial P}-\left(\bar{r}+\sum_{\substack{\jmath=1 \\ j \neq \imath}}^{N} \lambda_{\imath \jmath}^{Q}\right) V^{\imath}
$$

For $P \rightarrow \infty$, we apply the commonly used boundary condition $V_{P P}^{\imath}=0$ (Wilmott [1998]), which implies that

$$
V^{\imath} \simeq x(w, r, \tau) P+y(w, r, \tau) .
$$

The boundary PDE now is written as

$\frac{\partial V^{\imath}}{\partial \tau}=C_{1} V^{\imath}+B V^{\imath}+\sup _{z \in Z(r)}\left(z \frac{\partial V^{\imath}}{\partial r}\right)+H(r, w) a(\ell-r) \frac{\partial V^{\imath}}{\partial w}+H(r, w) q(r, h(w))(P-c) ; P=P^{\max }$

where the operators $C_{1}$ is given by

$$
C_{1} V^{\imath}=\left[\alpha^{\imath}\left(K^{\imath}-P\right)-\Lambda^{\imath} \sigma^{\imath} P\right] \frac{\partial V^{\imath}}{\partial P}-\left(\bar{r}+\sum_{\substack{j=1 \\ j \neq \imath}}^{N} \lambda_{\imath \jmath}^{Q}\right) V^{\imath}
$$

We solve the PDE in the region $P \in\left(0, P^{\max }\right)$. At the boundaries we solve PDE (3.25) at

\footnotetext{
${ }^{6}$ See Chen and Forsyth [2008] for more details.
} 
$P=0$ and $\mathrm{PDE}(3.26)$ at $P=P^{\max }$.

\subsection{Specification of the Empirical Example}

\subsubsection{Description of the Hydro Plant}

The empirical analysis in this study is done for a medium-sized hydro plant similar to the Abitibi Canyon generating station in north eastern Ontario owned by Ontario Power Generation. This station has a generation capacity of 336 MW. For comparison the Sir Adam Beck I and II generating stations at Niagara Falls have capacities of about 500 MW and 1,500 MW respectively and the Donzère-Mondragon Dam in France has an installed capacity of $354 \mathrm{MW}$. The combined physical capacity (CPC) of the generators at the Abitibi Canyon station is about 19 thousand Cubic-feet-per-second (CFS) and the storage capacity of the reservoir is about 17 thousand acre-feet. ${ }^{7}$

The analysis is conducted for a time horizon of one week ${ }^{8}$ which is a sufficient time scale for the questions addressed in this chapter. We abstract from the natural variations in water inflow and assume a constant inflow of $6,671 \mathrm{CFS},{ }^{9}$ which is the average hourly inflow rate based on data for the historical water inflow for the hydro station from 01 January 2001 to 30 November 2006. As noted earlier this assumption is considered realistic for the short time horizon we are using. A stochastic mean reverting diffusion water inflow process could be incorporated into this empirical study, which however would add another dimension to the HJB-PDE. The cost of generating hydroelectric power is assumed constant at $20 \mathrm{EUR} / \mathrm{MWh}$ for the hydro station.

\footnotetext{
${ }^{7}$ Detailed descriptions of the generating station can be found in Statistics Canada [2000], Hendry and Chang [2001], and http://www.opg.com/power/hydro/northeast_plant_group/abitibi.asp.

${ }^{8}$ In two similar studies, the time horizon is 15 days in Thompson et al. [2004] and one week in Chen and Forsyth [2008]. For the empirical studies in this chapter, the computational time varies with the level of ramping restrictions and takes from less than a day to one week.

${ }^{9}$ This only represents the average case in our sample period. The analysis for a specific season (wet or dry period) could be easily conducted by calculating the average hourly inflow rate in that period.
} 
We use a standard hydro power production function from the power engineering literature. The amount of electricity produced can be represented by

$$
q(r, w) \propto r \times h(r, w) \times e(r, h) .
$$

where $q$ is the power output, $r$ is the water release rate, $h$ is the gross head, $w$ is the water content, $e$ is the efficiency factor and $\propto$ means proportion. Under some simplifying assumptions about its functional form from Niu and Insley [2013], the following production function is used in our empirical analysis

$$
\begin{aligned}
q(r, h(w)) & =0.001 \times g \times r \times h(w) \times e \\
& =0.28 \times r \times h(w)
\end{aligned}
$$

where $g$ is the gravitational constant (32.15 feet-per-square-second) and the factor 0.001 converts $q$ to MW from KW, $r$ is in CFS and $h$ is in feet. The efficiency factor is assumed constant at 0.87 . We use a linear functional form between the head and water content in the reservoir, represented by

$$
h(w)=b \times w
$$

where $w$ is the water content in acre-feet and $b$ is assumed to be 0.0089 .

In the empirical analysis, the operational and environmental constraints and their associated values for the base case are specified as

- Up-ramping and down-ramping constraints are 3,000 Cubic-feet-per-second per hour (CFS-hr);

- The minimum water release requirement is 2,000 CFS and the maximum release constraint is 15,000 CFS;

- The minimum water content requirement is 7,000 acre-feet and the maximum value is 17,000 acre-feet;

These are similar to the cases examined in Niu and Insley [2013]. The input parameters 


\begin{tabular}{|c|c|c|c|}
\hline Parameter & Value & Parameter & Value \\
\hline$\ell$ & $6671 \mathrm{CFS}$ & $w^{\max }$ & 17000 acre-feet \\
\hline$g$ & 32.15 feet/square-second & $w^{\min }$ & 7000 acre-feet \\
\hline$\rho$ & 1000 kg/cubic-meter & $r^{\max }$ & 15000 CFS \\
\hline$b$ & 0.0089 & $r^{\min }$ & 2000 CFS \\
\hline$e$ & 0.87 & $q^{\max }$ & $336 \mathrm{MW}$ \\
\hline$C P C$ & $19000 \mathrm{CFS}$ & $q^{\min }$ & $0 \mathrm{MW}$ \\
\hline- & - & $r^{u}$ & 3000 CFS-hr \\
\hline- & - & $r^{d}$ & 3000 CFS-hr \\
\hline
\end{tabular}

for the hydro power production and hydro dam specifications are reported in Table 3.1.

\subsubsection{Specification of the Regime Switching Price Process}

We base our Markov regime switching model on the model estimated in Janczura and Weron [2009], which is parsimonious and can capture pertinent characteristics for electricity price dynamics. They use the German EEX spot price from 2001-2009 to estimate the following model ${ }^{10}$

$$
\begin{gathered}
d P=\eta\left(\mu_{1}-P\right) d t+\sigma_{1} \sqrt{P} d Z . \\
\log (P-m) \sim N\left(\mu_{2}, \sigma_{2}^{2}\right), P>m .
\end{gathered}
$$

where the base regime is the CIR (Cox-Ingersoll-Ross) process and the spike regime is the shifted lognormal distribution (with higher mean and variance than those in the base regime) which assigns zero probability to prices below the median $m$. Introducing heteroskedasticity through the CIR square root process accounts for the fact that electricity price volatility generally increases with price level, because positive price shocks tend to increase volatility more than negative shocks. The shifted spike regime distribution (lognormal) is used in order to correctly separate spikes from the 'normal' price behavior. Their empirical study shows that these specifications are more realistic for electricity spot

\footnotetext{
10 Janczura and Weron [2009] estimate the Markov regime switching models with shifted lognormal or Pareto spikes and Vasicek or CIR base regimes. The test results for the base regime show that the CIR specification is more suitable than the Vasicek specification and the $p$-values for the spike regime indicate that the lognormal distribution gives a better fit compared to the Pareto spike distribution.
} 
Table 3.2: Parameter Values Estimated by Janczura and Weron [2009]

\begin{tabular}{cc}
\hline Parameter & Value \\
\hline$\mu_{1}$ & $47.194 \mathrm{EUR} / \mathrm{MWh}$ \\
$\mu_{2}$ & 3.44 \\
$\eta$ & 0.36 \\
$\sigma_{1}$ & 0.73485 \\
$\sigma_{2}$ & 0.83066 \\
$m$ & $46.54 \mathrm{EUR} / \mathrm{MWh}$ \\
$\lambda_{12}$ & 0.0089 \\
$\lambda_{21}$ & 0.8402 \\
\hline
\end{tabular}

Base regime: $d P=\eta\left(\mu_{1}-P\right) d t+\sigma_{1} \sqrt{P} d Z$ Spike regime: $\log (P-m) \sim N\left(\mu_{2}, \sigma_{2}^{2}\right), P>m$.

prices and lead to better spike identification and goodness-of-fit than in the standard meanreverting Markov regime switching models (i.e. the Vasicek or Ornstein-Uhlenbeck process) with constant volatility dynamics.

Janczura and Weron [2009] obtain maximum likelihood estimates of the parameters of Equations (3.30) and (3.31) using the algorithm of Kim [1994]. The mean daily EEX spot price data is first deseasonalized by removing a long run seasonal trend representing changing climate and consumptions conditions during the year and long-term non-periodic structural changes. In addition a weekly periodic component is removed. Table 3.2 shows the parameter estimates obtained from Janczura and Weron [2009] for the 2001 to 2005 period of their sample. Note that since the estimation is done using daily data, the parameters can be interpreted using a daily time scale.

The model specified in Equations (3.30) and (3.31) is different than that shown in Equation (3.2). In particular the spike regime is not modelled as an Itô process. We adapt the Janczura and Weron [2009] model to conform to standard Itô processes in both regimes so that it can be incorporated into the HJB equation describing optimal hydro dam operation. Specifically it is assumed the regime switching process can be specified as follows for the base and spike regimes respectively:

$$
d P=\eta\left(\mu_{1}-P\right) d t+\sigma_{1} \sqrt{P} d Z+P\left(\xi^{12}-1\right) d X_{12} .
$$




$$
d P=\sigma_{2}(P-m) d z+P\left(\xi^{21}-1\right) d X_{21}, P>m
$$

The P-measure transition intensities are as defined in Equation (3.2).

Note that Equations (3.32) and (3.33) also include terms at the end which specify the jump in price level when a regime change happens. The spike regime is only defined for prices above the median, $m$. It was thus necessary to assume that when a change in regime happens from the base to the spike regime there will be a jump up in price level, with the jump size reflecting the ratio of the long run expected values in the base and spike regimes. When a transition from the spike to the base regime occurs, a jump down in price will occur at level of equal but opposite magnitude.

The parameters in Table 3.2 are estimated under the $\mathrm{P}$ measure (physical measure). To derive the option value using the hedging approach as shown in Appendix A.1.1, we require the market price of risk to convert the $\mathrm{P}$ measure to the $\mathrm{Q}$ measure. Under the $\mathrm{Q}$ measure Equations (3.32) and (3.33) becomes

$$
\begin{gathered}
d P=\left[\eta\left(\mu_{1}-P\right)-\Lambda_{1} \sigma_{1} \sqrt{P}\right] d t+\sigma_{1} \sqrt{P} d \hat{Z}+P\left(\xi^{12}-1\right) d \hat{X}_{12} . \\
d P=\sigma_{2}(P-m) d \hat{z}+P\left(\xi^{21}-1\right) d \hat{X}_{21}, P>m .
\end{gathered}
$$

where $d \hat{Z}$ and $d \hat{z}$ are the increment of the standard Gauss-Wiener processes under the $\mathrm{Q}$ measure specified by the choice of $\Lambda_{1}$ and $\Lambda_{2}$ respectively. $d \hat{X}_{12}$ and $d \hat{X}_{21}$ indicate the transition of the Markov chain under the $\mathrm{Q}$ measure. In this empirical study we rely on existing studies of the market price of risk to convert the drift from the physical measure to the risk neutral measure. However, as there are no current studies converting P-measure transition probabilities to Q-measure probabilities ${ }^{11}$, sensitivity analysis is employed to investigate the impact of changing transition probabilities.

The parameter values adopted for Equations (3.34) and (3.35) are shown in Table 3.3. The long run price, $\mu_{1}$, median price, $m$, mean reversion speed, $\eta$, and volatilities $\sigma_{1}$ and $\sigma_{2}$ are taken from the Janczura and Weron estimates given in Table 3.2. One problem with

\footnotetext{
${ }^{11}$ In practice, Q-measure probabilities can be calibrated to the observed prices of traded options for electricity prices.
} 
Table 3.3: Parameter Values for the Regime Switching Model

\begin{tabular}{cccc}
\hline Parameter & Value & Parameter & Value \\
\hline$\mu_{1}$ & $47.194 \mathrm{EUR} / \mathrm{MWh}$ & $\eta$ & 0.36 \\
$m$ & $46.54 \mathrm{EUR} / \mathrm{MWh}$ & $c$ & $20 \mathrm{EUR} / \mathrm{MWh}$ \\
$\sigma_{1}$ & 0.73485 & $\sigma_{2}$ & 0.83066 \\
$\xi^{12}$ & 1.6470 & $\xi^{21}$ & 0.6072 \\
$\lambda_{12}^{Q}$ & 0.0089 & $\lambda_{21}^{Q}$ & 0.8402 \\
$\Lambda_{1}$ & -0.2481 & $\Lambda_{2}$ & -0.2481 \\
$P_{1}^{\max }$ & $200 \mathrm{EUR} / \mathrm{MWh}$ & $P_{2}^{\max }$ & $200 \mathrm{EUR} / \mathrm{MWh}$ \\
$P_{1}^{\min }$ & $0 \mathrm{EUR} / \mathrm{MWh}$ & $P_{2}^{\min }$ & $48 \mathrm{EUR} / \mathrm{MWh}$ \\
$T$ & $168 \mathrm{~h}$ & $\bar{r}$ & $0.05 \mathrm{annually}$ \\
\hline
\end{tabular}

Base regime: $d P=\left[\eta\left(\mu_{1}-P\right)-\Lambda_{1} \sigma_{1} \sqrt{P}\right] d t+\sigma_{1} \sqrt{P} d \hat{Z}+P\left(\xi^{12}-1\right) d \hat{X}_{12}$. Spike regime: $d P=\sigma_{2}(P-m) d \hat{z}+P\left(\xi^{21}-1\right) d \hat{X}_{21}, P>m$.

using these estimates is that our empirical analysis involves hourly control of the ramping rate. However the parameters from Janczura and Weron [2009] are estimated with mean daily data. Use of daily averages in this context is clearly not ideal. Any regular daily patterns are ignored. The chapter undertakes sensitivities to investigate the impact of these parameter estimates. Estimating parameter values on an hourly basis will be a topic for future research.

As noted above $\xi^{12}$ is set equal to the ratio of the spike regime mean price to the base regime mean price. $\xi^{21}$ is roughly the inverse of $\xi^{12}$. So, on average $\xi^{21} \times \xi^{12}$ is around 1 to avoid arbitrage opportunities. $\lambda_{12}^{Q}$ and $\lambda_{21}^{Q}$ represent the regime transition intensities in the risk neutral world. The risk adjusted probability of switching from regime $\imath$ to regime $j$ is $\lambda_{i j}^{Q} d t$. As expected the probability of remaining in the base regime over a single day is very high, so that the spike regime occurs quite rarely. As was noted earlier, these estimated transition probabilities ${ }^{12}$ from Janczura and Weron [2009] are under the P-measure and we use them as the risk neutral transition probabilities in our empirical study, which is problematic. However, in the sensitivity analysis we will check the robustness of our results by assuming different transition probabilities.

\footnotetext{
${ }^{12}$ In the literature, constant transition probabilities are usually estimated and used for derivative pricing and risk management. However, this specification may not be appropriate in our asset pricing model as the transition probability for electricity prices should not be constant, but depends on some observable variables such as the demand level.
} 
Another important parameter is the market price of risk $\Lambda$, representing the excess return over the risk-free rate that the market expects for taking extra risks. The empirical evidence for the market price of risk in the power markets are mixed in terms of the magnitude, variability and sign which could be positive or negative. It generally varies over time and across markets. Some of the empirical estimations are provided by Lucia and Schwartz [2002], Cartea and Figueroa [2005], Kolos and Ronn [2008] and Weron [2008]. In Weron [2008] the market prices of risk implied from AEO-GB0300 Asian-style options and GB0300 futures ranges from -0.2 to 0.1 during the period November 1, 1999 - January 28,2000 . In this chapter we assume the market price of risk is a deterministic constant ${ }^{13}$ and use the estimated value from Cartea and Figueroa [2005], where they use historical spot data and forward data from the England and Wales electricity market and find the market price of risk is -0.2481. As Weron [2008] explains "negative value is a higher incentive for hedging on the demand side relative to the supply side, because of the non-storability of electricity as compared to the (limited and costly but still existent) storage capabilities of fuel".

Table 3.3 also shows the assumed variable cost of electricity production, $c$, the upper and lower limits on price in the numerical analysis, $P_{\imath}^{\min }$ and $P_{\imath}^{\max }, \imath=1,2$, the timeframe for the analysis in hours, $T$, and the risk free rate, $\bar{r}$.

\subsection{Empirical Results}

\subsubsection{Base Case}

In this section, we present the numerical results for the base case with ramping constraints at 3,000 CFS-hr. The value of the hydro power station is a function of electricity price, water content, release rate, time and regime. We are interested in how these variables will affect the profit and operation of the power plant under various levels of ramping restrictions. Due to the high dimensionality of the value function, we can only analyze the

\footnotetext{
${ }^{13}$ We could specify the market price of risk that is a linear or nonlinear function of the state variables. Then the estimated time-varying market price of risk could be incorporated into the HJB-PDE.
} 
results by looking at various combinations of the variables while holding other variables constant. So, given any ramping restrictions, for the numerical results we will focus on the following relationships: power plant value versus price and release rate; optimal ramping rate versus price and release rate; optimal ramping rate versus price and time; power plant value versus price and reservoir level; optimal ramping rate versus price and reservoir level.

Figures 3.3(a) through 3.7(b) show the results for this regime switching case when the constraints for ramping rate, water release rate and water content are imposed. Figures 3.3(a) and 3.3(b) plot the value of the hydro power plant as a function of water release rate and spot price at time zero for the base regime and the spike regime respectively when the reservoir level is at the full capacity and the inflow rate is at 6,671 CFS. Note that the spike regime is only defined for prices above $46.54 \mathrm{EUR} / \mathrm{MWh}$ while the base regime is defined over the complete range shown from 0 to $200 \mathrm{EUR} / \mathrm{MWh}$. In the base regime we observe that the value of the hydro power plant is increasing with the spot price. However, the hydro power plant's value is increasing with the water release rate for prices above the marginal cost of electricity generation of $20 \mathrm{EUR} / \mathrm{MWh}$ and decreasing with the water release rate for prices below the marginal cost of electricity generation. This is due to the fact that the power plant makes losses by generating power when the spot price moves below the marginal cost. But in the spike regime the value of the hydro power plant is increasing with both water release rate and spot price. Not surprisingly, at any given water release rate and spot price, the value of the hydro power plant in the spike regime is higher than its value in the base regime. More specifically, in the base regime the value reaches the maximum of EUR 1,541,400 when the current release rate is 15,000 CFS and the price is $200 \mathrm{EUR} / \mathrm{MWh}$ and its value reaches the minimum of EUR 1,282,100 when the current release rate is 15,000 CFS and the price is $0 \mathrm{EUR} / \mathrm{MWh}$. Even at a price of $0 \mathrm{EUR} / \mathrm{MWh}$ the power plant's value is still positive due to the fact that the price will move up at a later time and generate positive profits. Similarly, in the spike regime the value reaches the maximum of EUR 1,580,300 when the current release rate is 15,000 CFS and the price is $200 \mathrm{EUR} / \mathrm{MWh}$ and its value reaches the minimum of EUR 1,340,900 when the current release rate is 2,000 CFS and the price is $48 \mathrm{EUR} / \mathrm{MWh}$.

Figures 3.4(a) and 3.4(b) show the optimal control strategies corresponding to Figures 
3.3(a) and 3.3(b) respectively. In general, for the base regime the power plant will ramp up at the maximum limit of 3,000 CFS-hr to generate more power and make higher profits if the price is high and ramp down at the maximum limit of 3,000 CFS-hr to accumulate more water in the reservoir for generation at later time if the price is low. However the constraints on release rates and reservoir size also have an impact on optimal operations, leading to some complex interactions. In Figure 3.4(a) four regions have been labelled. For example, I denotes a region where the release rate is high but prices are low. The optimal action is to ramp down at the maximum rate of 3,000 CFS-hr. This tells us that the high release rate is non-optimal at these low prices, and ramping down allows the reservoir to replenish. In Region II prices are low and the release rate is at or near the minimum. The operator must ramp up to avoid violating the minimum release constraint. However as the release rate is increased, but remaining at low prices we see a switch in the optimal action from ramping up to ramping down. In general, ramping down is preferable when prices are low whenever it is possible, given other constraints. Region III shows an area of high prices and low release rates, and it makes sense that the optimal action is to ramp up. Finally Region IV indicates an area which has a high release rate and high prices as well. The constraint on the maximum release rate prevents ramping up at the maximum rate in this region. For the spike regime, since the price is much higher the power station will ramp up at 3,000 CFS-hr whenever possible given the other constraints. If we truncate Figure 3.4(a) for prices below $48 \mathrm{EUR} / \mathrm{MWh}$, the optimal control strategies for the base regime and the spike regime are very similar, except the controls in the low price and high release rate region where the boundaries for ramping up and ramping down are slightly different for these two regimes. 


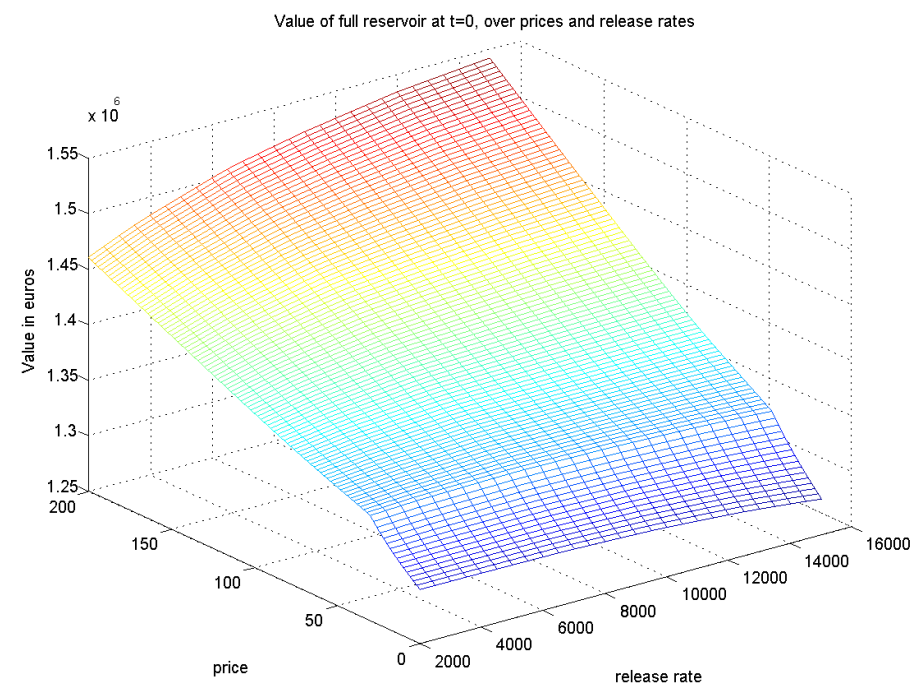

(a) Value over Price and Release Rate for the Base Regime

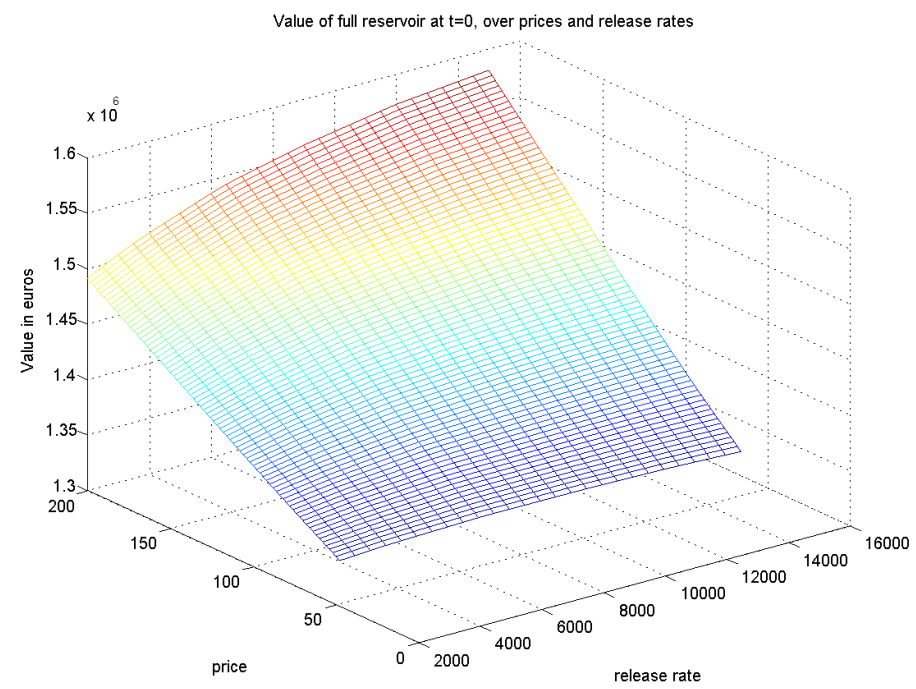

(b) Value over Price and Release Rate for the Spike Regime

Figure 3.3: Value over Price and Release Rate for the Base and Spike Regimes 


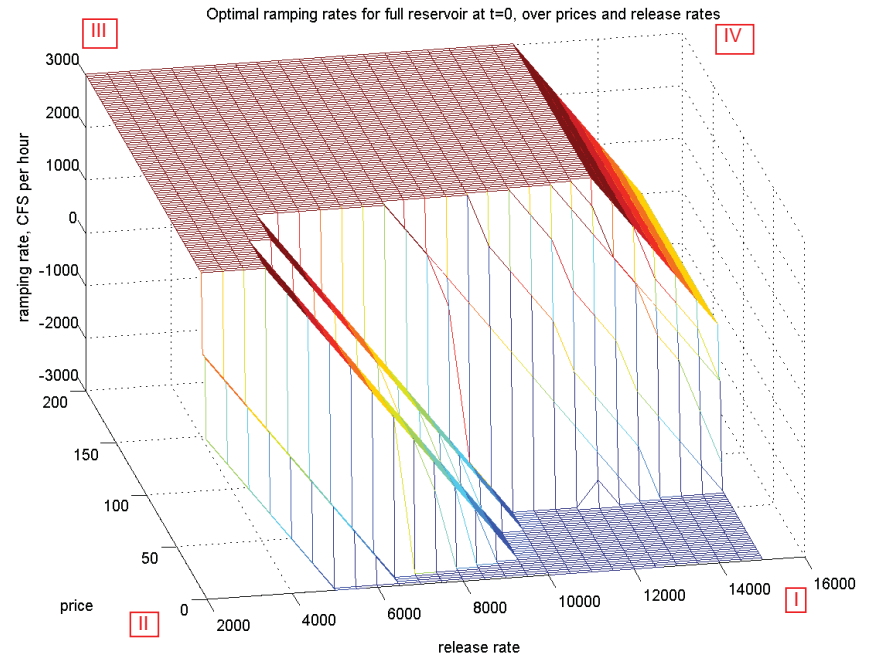

(a) Optimal Ramping Rate over Price and Release Rate for the Base Regime

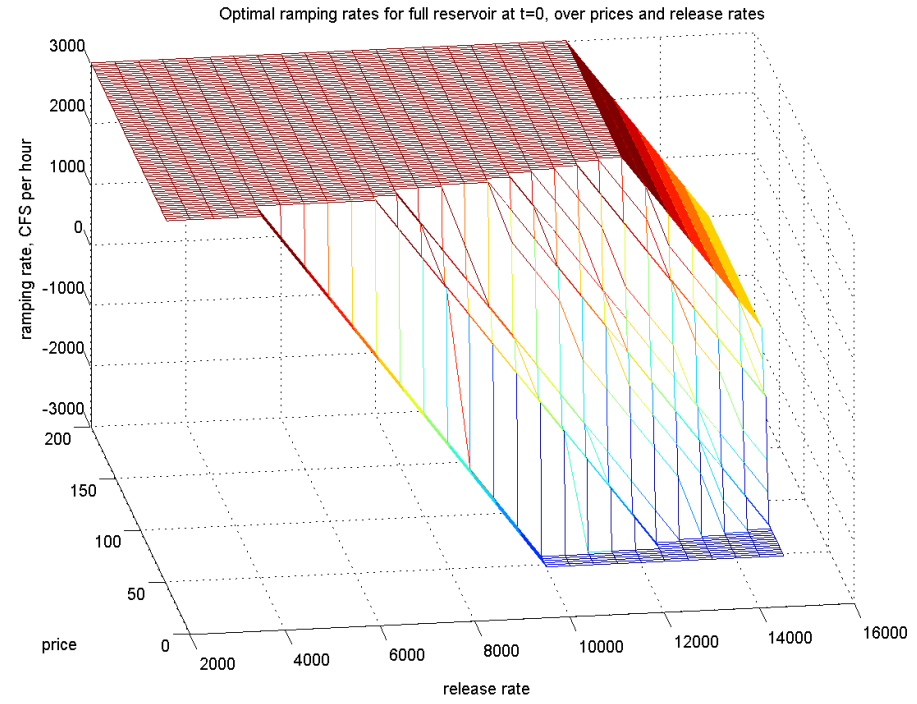

(b) Optimal Ramping Rate over Price and Release Rate for the Spike Regime

Figure 3.4: Optimal Ramping Rate over Price and Release Rate for the Base and Spike Regimes 


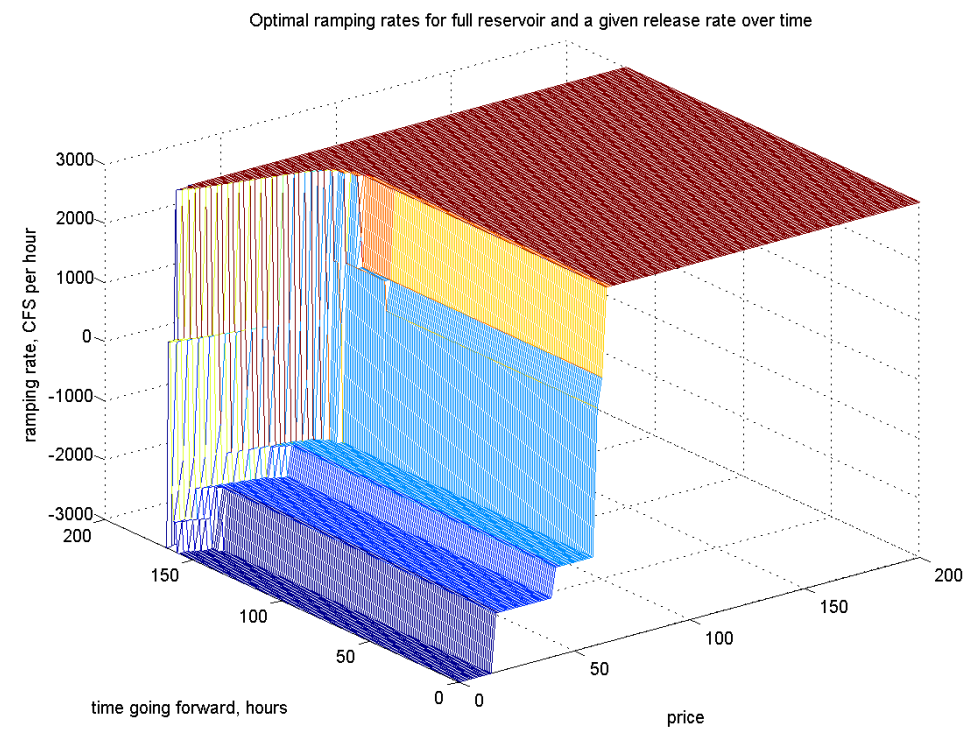

(a) Optimal Ramping Rate over Price and Time for the Base Regime

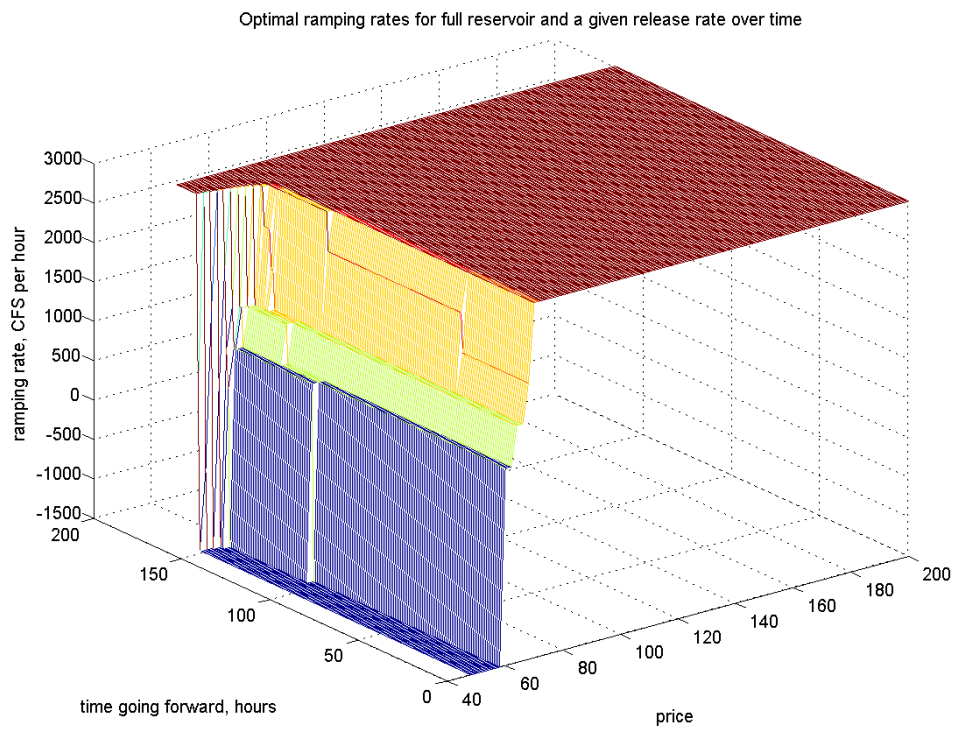

(b) Optimal Ramping Rate over Price and Time for the Spike Regime

Figure 3.5: Optimal Ramping Rate over Price and Time for the Base and Spike Regimes 
Figures 3.5(a) and 3.5(b) plot the optimal operational strategies for the hydro power plant as a function of time and spot price for the base regime and the spike regime respectively when the reservoir level is at the full capacity, the water release rate is at 8,500 CFS which is midway between the upper and lower limits. These diagrams allow a better view of the impact of price on the optimal strategy and also indicate the effect of imposing a terminal time of one week. For the base regime we can clearly observe that at lower prices it is optimal to ramp down and preserve the water in the reservoir, whereas for high prices it is optimal to ramp up thereby increasing the release rate and power generation. More specifically, for a typical hour there are four operational regions: the power plant will ramp down at the maximum limit of 3,000 CFS-hr in order to generate less power and keep the reservoir at full capacity if the price is below $20 \mathrm{EUR} / \mathrm{MWh}$; if the price lies between 20 EUR/MWh and $40 \mathrm{EUR} / \mathrm{MWh}$ the station will ramp down at 2,000 CFS-hr; when the price is between $40 \mathrm{EUR} / \mathrm{MWh}$ and $60 \mathrm{EUR} / \mathrm{MWh}$ the station will ramp down at 1,500 CFS-hr; as the price moves above $60 \mathrm{EUR} / \mathrm{MWh}$ the station will ramp up at the maximum limit of 3,000 CFS-hr. It may also be observed that the optimal control strategy remains largely unchanged over time, but behaves quite differently when the time moves close to the terminal time. Since the terminal boundary condition of zero value is imposed, the power plant will ramp up at the maximum allowed rate when time is close to the end of the time horizon. For the spike regime the station will mostly ramp up at the maximum limit of 3,000 CFS-hr due to higher prices.

Figures 3.6(a) and 3.6(b) plot the value of the hydro power plant as a function of reservoir level and spot price at time zero for the base regime and the spike regime respectively when the release rate is at $8,500 \mathrm{CFS}$. In both regimes we observe that the value of the hydro power plant is increasing with both the reservoir level and spot price. At any given reservoir level and spot price, the value of the hydro power plant in the spike regime is higher than its value in the base regime. More specifically, in the base regime the value reaches the maximum of EUR 1,517,400 when the current reservoir level is 17,000 acre-feet and the price is $200 \mathrm{EUR} / \mathrm{MWh}$ and its value reaches the minimum of EUR 1,162,600 when the current reservoir level is 7,000 acre-feet and the price is 0 EUR/MWh. Similarly, in the spike regime the value reaches the maximum of EUR 1,554,800 when the current 
reservoir level is 17,000 acre-feet and the price is $200 \mathrm{EUR} / \mathrm{MWh}$ and its value reaches the minimum of EUR 1,194,200 when the current reservoir level is 7,000 acre-feet and the price is $48 \mathrm{EUR} / \mathrm{MWh}$.

Figures 3.7(a) and 3.7(b) show the optimal control strategies corresponding to Figures 3.6(a) and 3.6(b) respectively. For the base regime, in the lower reservoir boundary region there are two cases. First, if the price is low it is optimal to ramp down at the maximum limit of 3,000 CFS-hr to let the reservoir accumulate more water. Second, if the price is high enough it is even optimal to ramp up at the maximum limit of 3,000 CFS-hr to make more profits, but this is not sustainable since it tends to violate the lower water content constraint. However, if the initial water content is anywhere above the minimum water content region, this will not cause any concerns. In fact, it is never optimal to operate anywhere close to the lower reservoir boundary since the power plant's value is positively related to the water content as shown in Figure 3.6(a). In addition, for any water content above the lower reservoir boundary there is an operational boundary between up ramping at maximum limit of 3,000 CFS-hr and down ramping at maximum limit of 3,000 CFS-hr. In the low price and full reservoir region it is optimal to ramp down at rates lower than the maximum allowed rate to keep the reservoir full. Furthermore, in the low price region, for any water content below the maximum level the dam will ramp down at the maximum allowed rate to let the reservoir store more water even though it could make profits at the current operating mode. For the spike regime, the optimal control strategies are similar to the base regime for prices above $48 \mathrm{EUR} / \mathrm{MWh}$. We notice that in the high price and lower reservoir boundary it is also optimal to ramp up at the maximum limit of 3,000 CFS-hr. However, because the price will quickly switch back to the base regime in this case the plant will again ramp down at the maximum limit of 3,000 CFS-hr to let the reservoir accumulate more water and avoid violating the lower water content constraint.

\subsubsection{Impact of Changing Ramping Restrictions}

In this section, we analyze the implication of the ramping constraints on the value of the hydro power plant. The impact of these constraints on profits for a range of ramping 
constraints is detailed in Table 3.4, which is represented by Figures 3.8(a) and 3.8(b) graphically. Figure 3.8(a) plots the case at half release rate and full reservoir level and Figure 3.8(b) plots the case at full release rate and full reservoir level. In general, the more restrictive the constraint the greater is the limitation on the station's operational flexibility and the larger the impact on profits. However for this particular example profit is not highly sensitive to ramping rate restrictions. Under the same conditions, higher initial electricity price always gives higher present value.

Without ramping restrictions the total profit from providing power in the base regime is EUR 1,367,600 when the initial price is $40 \mathrm{EUR} / \mathrm{MWh}$, the reservoir level is at the full capacity, the water release rate is at the upper limit and the inflow rate is at 6,671 CFS. With ramping constraints of 5,000 CFS-hr, the total profit drops to EUR 1,361,400, representing a $0.5 \%$ reduction over the scenario without ramping restrictions. As ramping rate restrictions are increased profits continue to drop, until at a restriction of $250 \mathrm{CSF}-\mathrm{hr}$, then the total profit drops to as low as EUR 1,254,100, which is a $8.3 \%$ decrease relative to the scenario without ramping restrictions. The reduction of profit in this most restrictive scenario is similar to what is reported in Niu and Insley [2013] in a deterministic framework in which they assume each day is divided into peaking and off peaking regimes. In Niu and Insley [2013] the value of the hydro operation is reduced by $6.9 \%$ under the most severe ramping restrictions. Keeping other conditions the same, if the initial release rate is half of the upper limit, then the total profit drops to EUR 1,310,700, which is only a $4.3 \%$ decrease relative to the scenario without ramping restrictions. From Figures 3.8(a) and 3.8(b) we also observe profits fall proportionately more as ramping restrictions are increased when ramping rates are already quite restrictive - i.e. for rates of less than 1,000 CFS-hr. Similar results are obtained under other specifications such as different initial price, water release rate and regime. It is noticed that even if the price jumps to $160 \mathrm{EUR} / \mathrm{MWh}$ in the spike regime, at the most restrictive ramping constraint of $250 \mathrm{CSF}$-hr the total profit only drops $5.9 \%$ over the scenario without ramping restrictions.

One concern regarding Janczura and Weron [2009]'s estimation is that they model daily average electricity price, instead of the hourly electricity price. We would expect the daily price spikes and volatility will be smaller than those values for the hourly price, but the 


\section{Table 3.4: Numerical Results Under Various Ramping Restrictions}

\begin{tabular}{|c|c|c|c|c|c|c|}
\hline Case & & $\begin{array}{l}\text { No Ramping } \\
\text { Restrictions }\end{array}$ & 5000 (CFS-hr) & 3000 (CFS-hr) & 1000 (CFS-hr) & 250 (CFS-hr) \\
\hline \multirow[t]{2}{*}{$\mathrm{HF}$} & Profit & 1368900 & 1364000 & 1355500 & 1339800 & 1310700 \\
\hline & $\%$ ch & $\mathrm{N} / \mathrm{A}$ & -0.4 & -1.0 & -2.1 & -4.3 \\
\hline \multirow[t]{2}{*}{$\mathrm{FF}$} & Profit & 1367600 & 1361400 & 1350700 & 1318000 & 1254100 \\
\hline & $\% \mathrm{ch}$ & $\mathrm{N} / \mathrm{A}$ & -0.5 & -1.2 & -3.6 & -8.3 \\
\hline \multicolumn{7}{|c|}{ Total Profit and Change of Total Profit in Regime 2 at Time 0 When the Initial Price is $80 \mathrm{EUR} / \mathrm{MWh}$} \\
\hline \multirow[t]{2}{*}{ HF } & Profit & 1401100 & 1395300 & 1385900 & 1367500 & 1337200 \\
\hline & $\% \mathrm{ch}$ & $\mathrm{N} / \mathrm{A}$ & -0.4 & -1.1 & -2.4 & -4.6 \\
\hline \multirow[t]{2}{*}{ FF } & Profit & 1403600 & 1397700 & 1387700 & 1358000 & 1298800 \\
\hline & $\% \mathrm{ch}$ & $\mathrm{N} / \mathrm{A}$ & -0.4 & -1.1 & -3.2 & -7.5 \\
\hline \multicolumn{7}{|c|}{ Total Profit and Change of Total Profit in Regime 2 at Time 0 When the Initial Price is $160 \mathrm{EUR} / \mathrm{MWh}$} \\
\hline \multirow[t]{2}{*}{$\mathrm{HF}$} & Profit & 1517300 & 1509700 & 1497400 & 1467700 & 1428500 \\
\hline & $\%$ ch & $\mathrm{N} / \mathrm{A}$ & -0.5 & -1.3 & -3.3 & -5.9 \\
\hline \multirow[t]{2}{*}{$\mathrm{FF}$} & Profit & 1529600 & 1524100 & 1514900 & 1490300 & 1449000 \\
\hline & $\% \mathrm{ch}$ & $\mathrm{N} / \mathrm{A}$ & -0.4 & -1.0 & -2.6 & -5.3 \\
\hline
\end{tabular}

Note: HF means half release rate and full reservoir level; FF means full release rate and full reservoir level. Profit is in Euros and \%ch refers to the percent change in profits compared to the case of no ramping restrictions.

daily mean reverting rate will be higher than the mean reverting rate for the hourly price. These could cause an under valuation of the hydro power station. In addition, any regular daily trends are ignored. This shortcoming in the parameter estimates could also affect the results about the impact of ramping restrictions on hydro plant's value and operation. To check the robustness of our results, it is necessary to conduct a sensitivity analysis. 


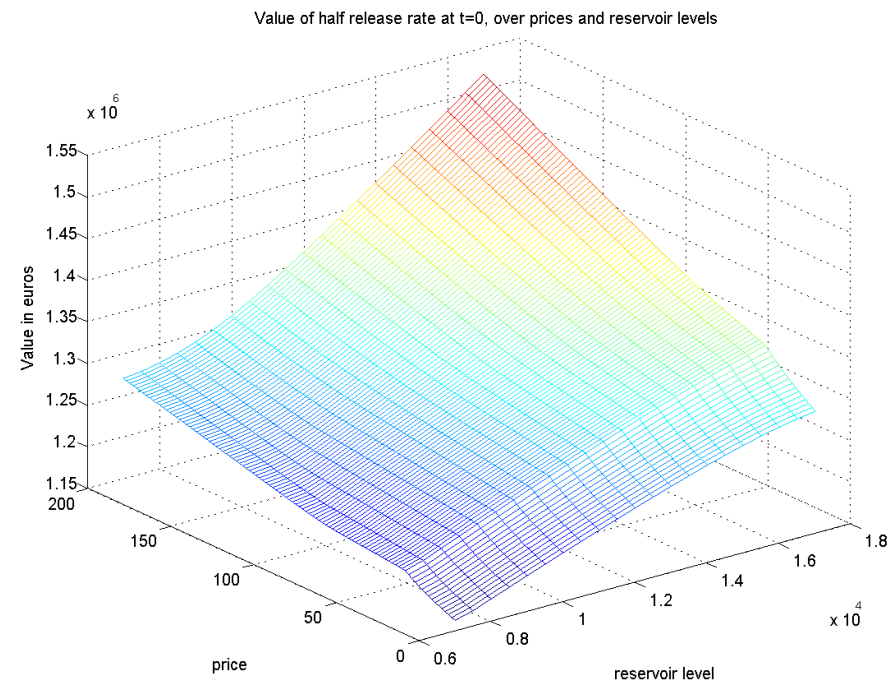

(a) Value over Price and Reservoir Level for the Base Regime

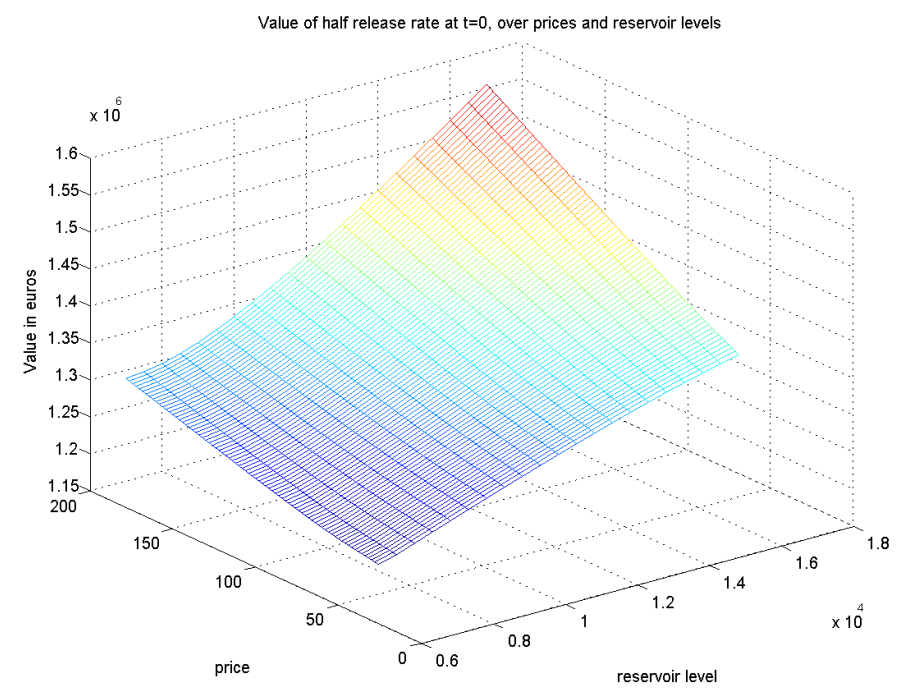

(b) Value over Price and Reservoir Level for the Spike Regime

Figure 3.6: Value over Price and Reservoir Level for the Base and Spike Regimes 


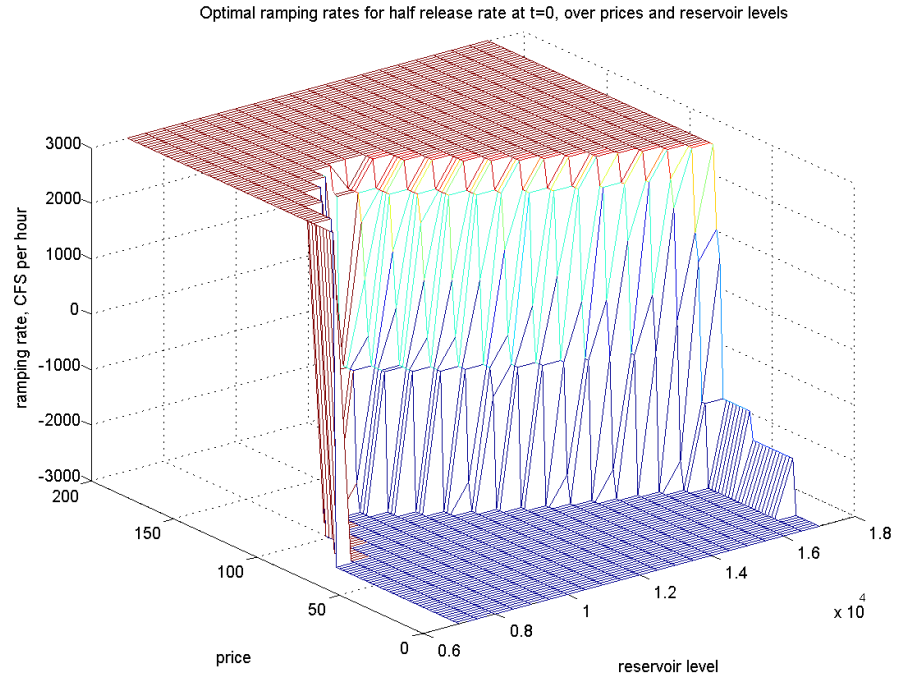

(a) Optimal Ramping Rate over Price and Reservoir Level for the Base Regime

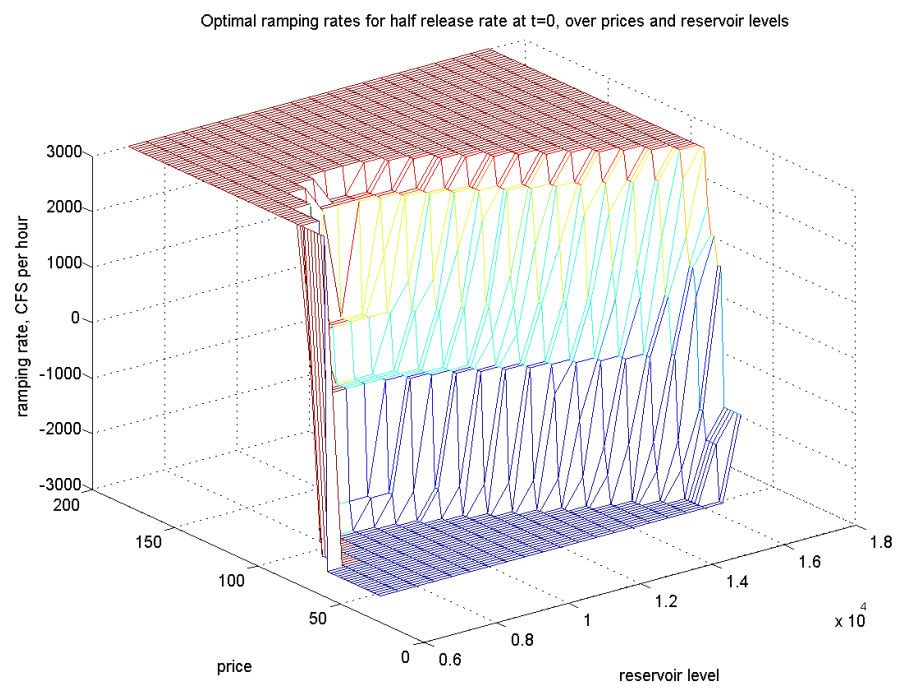

(b) Optimal Ramping Rate over Price and Reservoir Level for the Spike Regime

Figure 3.7: Optimal Ramping Rate over Price and Reservoir Level for the Base and Spike Regimes 


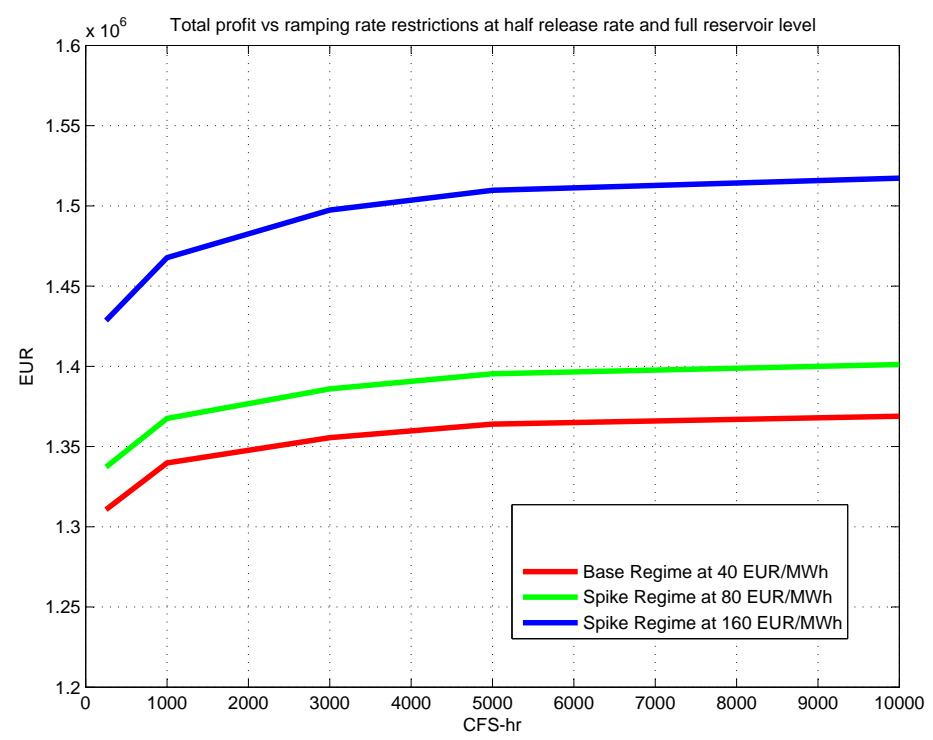

(a) Total Profit vs Ramping Rate Restrictions at Half Release Rate and Full Reservoir Level

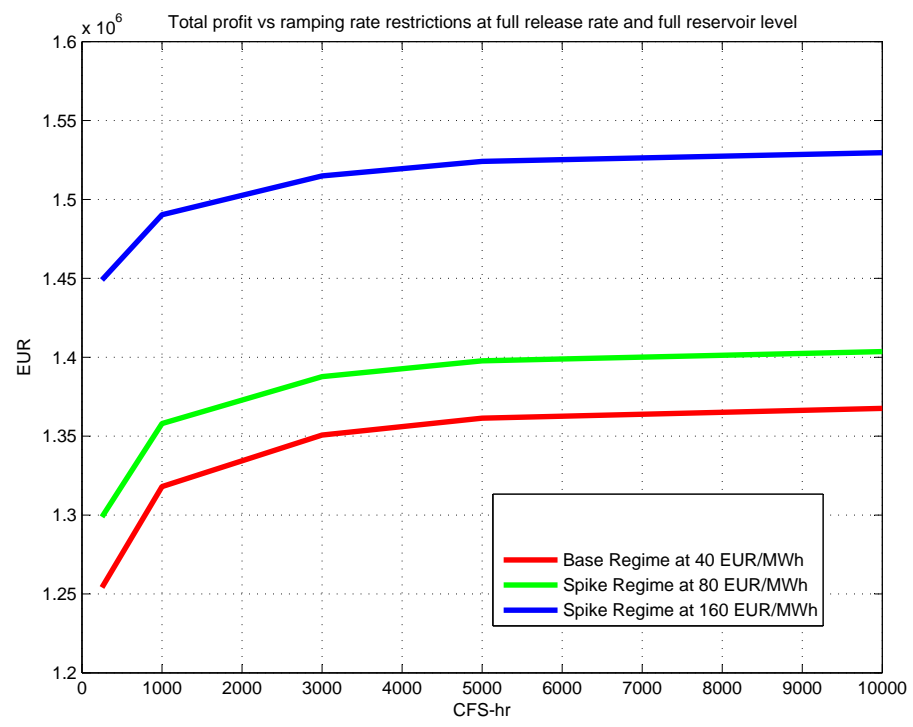

(b) Total Profit vs Ramping Rate Restrictions at Full Release Rate and Full Reservoir Level

Figure 3.8: Total Profit vs Ramping Rate Restrictions at Half Release Rate and Full Reservoir Level and at Full Release Rate and Full Reservoir Level 


\subsubsection{Sensitivity Analysis}

Due to the large number of parameters for the regime switching electricity price model (Table 3.3), we only conduct sensitivity analysis for five key parameters of interest: the speed of mean reversion in the base regime, the transition intensities in each regime, the volatilities, the long run mean in the base regime, and the cost of generating hydroelectric power. A sixth sensitivity is conducted to determine the significance of including the spike regime. In this case the base regime is modelled as a single regime.

For the first sensitivity, the speed of mean reversion is doubled from 0.36 to 0.72 . The results are shown in Table A.1 (Appendix A.2). The impact of ramping restrictions can be seen to be similar to the benchmark case. Ramping restrictions affect the profits negatively and profits fall proportionately more as ramping restrictions are increased when ramping rates are already quite restrictive. However for this higher speed of mean reversion the value of the hydro plant is lower, and the impact on profits of increasing ramping rates is slightly smaller. At the most restrictive ramping restrictions of $250 \mathrm{CFS}-\mathrm{hr}$ the profit drops by $7.6 \%$ in the worst scenario compared to the case of mean reverting rate at 0.36 , where the profit decreases by $8.3 \%$ in the worst scenario. The higher $\eta$ implies that price stays closer to the long run mean, which provides less opportunity for the hydro plant owner to benefit from an upward movement in price by ramping up and selling more power. Since the motivation to ramp up (or down) will be reduced, ramping restrictions would be expected to have a smaller impact.

The next sensitivity involves changing the transition probabilities by increasing the

probability that the price is in the jump regime. Specifically, $\lambda_{12}^{Q}$ is increased from 0.0089 to 0.02 and $\lambda_{21}^{Q}$ is decreased from 0.8402 to 0.7402 while keeping $\eta$ at 0.36 . The results are shown in Table A.2 in Appendix A.2. Again, we observe similar patterns for the impact of ramping restrictions in these two cases. But with the new transition probabilities the value is uniformly higher and the ramping impact is slightly larger (but at the same order of magnitude). At the most restrictive ramping restrictions of $250 \mathrm{CFS}-\mathrm{hr}$ the profit drops by $9.5 \%$ in the worst scenario compared to the benchmark case where the profit decreases by $8.3 \%$ in the worst scenario. The change in transition probabilities implies more time is 
spent in the spike regime, which as expected increases the value of the power station. But with a greater likelihood of being in the spike regime, the imposition of ramping restrictions limiting the ability to respond to higher prices has a larger impact.

For the third sensitivity, the volatilities for both regimes are increased. In the base regime the volatility, $\sigma_{1}$ is increased from 0.73485 to 0.93 . In the spike regime $\sigma_{2}$ is increased from 0.83066 to 1.43 . The other parameters are kept at benchmark case values. The results are given in Table A.3 in Appendix A.2. Compared to Table 3.4, we again observe the same patterns for the impact of ramping restrictions, but the higher volatilities cause two major changes. First, the value of the hydro plant is uniformly higher. This is consistent with the standard result on options pricing, i.e. higher volatility is associated with higher options value. Second, for higher volatilities the impact of ramping restrictions on the power plant's value is slightly larger (but at the same order of magnitude). As Table A.3 shows at the most restrictive ramping restrictions of 250 CFS-hr the profit drops by $9.3 \%$ in the worst scenario compared to the case of low volatilities, where the profit decreases by $8.3 \%$ in the worst scenario. This is caused by the fact that a higher volatility results in more frequent high prices and low prices. So these restrictive ramping restrictions affect the plant's ability to capture profits by ramping up or down quickly in response to more frequent price changes.

For the fourth sensitivity, the long run mean price for the base regime is decreased from 47.194 EUR/MWh to 27.194 EUR/MWh. The results are reported in Table A.4 in Appendix A.2. Compared to the benchmark case, we find similar patterns for the impact of ramping restrictions. However, a $20 \mathrm{EUR} / \mathrm{MWh}$ reduction of the base regime mean price results in a significantly lower value for the power plant. This is caused by the much smaller net profit under this lower mean price. In addition, for a lower mean price the impact of ramping restrictions on the power plant's value is larger. At the most restrictive ramping restrictions of $250 \mathrm{CFS}-\mathrm{hr}$ the profit drops by $13 \%$ in the worst scenario compared to the benchmark case where the profit decreases by $8.3 \%$ in the worst scenario. This is due to the fact that a lower mean price for the base regime increases the probability that the price falls below the cost of production. So these restrictive ramping restrictions limit the plant's ability to avoid negative profit by ramping down quickly in response to more 
frequent low prices.

For the fifth sensitivity, the cost of generating hydroelectric power is halved from 20 EUR/MWh to 10 EUR/MWh. The results are given in Table A.5 in Appendix A.2. The impact of ramping restrictions is very similar to the benchmark case. However for this lower cost of production the value of the hydro plant is significantly higher, and the impact on profits of increasing ramping rates is slightly smaller. At the most restrictive ramping restrictions of 250 CFS-hr the profit drops by $7.6 \%$ in the worst scenario compared to the case with cost at $20 \mathrm{EUR} / \mathrm{MWh}$, where the profit decreases by $8.3 \%$ in the worst scenario. A lower production cost means a higher net profit and higher power plant value. Since this lower cost also reduces the likelihood of negative profit, ramping restrictions would be expected to have a smaller impact.

In the empirical study above, we examine 6 cases: the benchmark case (Table 3.4) and 5 cases (Tables A.1 through A.5) from the sensitivity analysis. The results for the sensitivity analysis are also summarized in Table A.6 (Appendix A.2). The results show that ramping rates will have a larger effect when the price process is one in which more frequent ramping up or down is desirable - such as with higher volatilities or more frequent price spikes. In the empirical example in this chapter, price is mostly above the variable cost of producing power. This is why ramping restrictions do not have an overly severe effect on profits even at the most restrictive level. If the price process were such that price fell below cost frequently, then we expect that ramping restrictions would have a much more noticeable effect since the hydro operator would be unable to quickly respond to the low price by ramping down in order to decrease power supply.

In the final sensitivity the base regime is adopted as a single regime. This is done to determine the importance of the spike regime to the value and optimal operations of the hydro plant. The results are shown in Tables A.7, A.8, A.9, and A.10 in Appendix A.2. As expected, the single regime case gives an overall lower value than the two regime case. However the results are surprisingly close, differing only by 2 to 3 percent. In addition in the single regime case, ramping restrictions have a smaller impact than in the two regime case. However the change is again quite small, with the most severe restrictions reducing profits by $7.5 \%$ in the single regime case compared to $8.3 \%$ in the two regime case. 
Restrictive ramping constraints will limit the power plant's ability to make profits during price spikes, therefore the power plant's value is more severely affected when the spike regime is included. However, price spikes are still rare events. In addition if the hydro plant is already operating at full capacity when a spike occurs, no ramping is needed for the plant to capture the extra profits from the price spike.

These and other additional numerical experiments show that the ramping effect is largely determined by the level of the price relative to the cost of generation, i.e., how long and how frequent the price is close to or lower than the cost of hydro power, and is less sensitive to the price jumps. In other words, in Janczura and Weron [2009] where the electricity price is almost surely higher than the cost of power generation, the results show that the effect of ramping restrictions on the power plant's ability to gain the profit in the spike regime is minimal. This is because the hydro operation will be optimally operating somewhere close to the maximum release rate most of the time. Even though the price jumps frequently, it is still a rare event compared to the base regime. In Janczura and Weron [2009] the unconditional probability of the spike regime is as low as 0.0104 .

This exercise emphasizes the importance of accurately modelling electricity prices in order to obtain an accurate estimation of the impact of ramping restrictions on hydro station's value and operation. However, overall the results are quite robust and we can draw some general conclusions based on this sensitivity analysis. In the empirical example, it has been found that profits are significantly affected (by less than 10\%) in the case of the most severe ramping constraints. However we also find a range of less severe ramping constraints for which profits are impacted by less than $3 \%$.

\subsection{Conclusions}

The accurate modelling of electricity prices is still a topic of considerable debate in the literature. Markov regime switching models are becoming more popular as a means of capturing in a parsimonious manner the major characteristics of electricity prices such as price spikes. This chapter contributes to the literature by demonstrating the effect of using 
a regime switching model to analyze ramping rate issues in a prototype hydro power plant.

For a prototype hydro dam we valued the power plant and modelled the lost profits for a range of ramping restrictions for the regime switching model over a one week period. In most scenarios the optimal control is of a "bang-bang" type, i.e., ramping up at the upper limit when prices are high and ramping down at the upper limit when prices are low. The exception to this is when the dam is up against one of the other constraints such as maximum/minimum release rates or maximum water content in the dam. Profits are negatively affected by ramping restrictions. We find that profits are significantly affected (by less than 10\%) in the case of the most severe ramping constraints. However we also find a range of less severe ramping constraints for which profits are impacted by less than $3 \%$. In addition, by using the base regime in the regime switching model as the single regime, the results show that introducing the spike regime increases the value of the power plant and also increases the impact of ramping restrictions, but in our empirical example this impact is not very sensitive to the spike regime.

All of these results will depend on the specifics of the particular hydro plant under consideration such as the physical structure as well as the market structure that the plant operates in such as the pricing system. Nevertheless, the sensitivity analysis shows that these empirical findings are quite robust for this regime switching model for electricity prices. Notwithstanding price spikes have a significant effect on the value of the hydro power plant, one conclusion is that the ramping effect on profits mostly depends on the level of the price relative to the cost of generation (how long and how frequent the price is close to or below the cost), but is not very sensitive to the price jumps. In general ramping restrictions have a larger effect in an environment where frequent ramping up or down is desired. A lower speed of mean reversion, higher volatility and more frequent transition to the spike regime all have the effect of increasing the impact of ramping restrictions.

An important conclusion of the chapter is that ramping restrictions should not be determined in isolation, but rather using a cost-benefit approach that evaluates the trade offs involved. This chapter has identified some of the important trade offs that should be examined more carefully in future research. These include the impact on hydro plant's operation and profit. 
There are several directions for further research. First, we could account for uncertainty in water inflow assuming it could be modelled as mean reverting stochastic processes, but this would add another dimension to the HJB-PDE and obtaining the numerical solution for this problem is nontrivial. Second, this chapter adapted electricity process parameter estimates found in the literature. Further work is needed in estimating an electricity price model using hourly data, preferably in the Q-measure to avoid the necessity of determining an appropriate market price of risk. Third, further efforts are needed to construct a measure of the environmental benefits for the river ecosystem gained by imposing these ramping restrictions. Finally, the ramping issue could be better studied through a partial equilibrium model of hydro-thermal competition. Then we could analyze the impact of ramping restrictions on the electricity price, production, transmission, associated pollutant emission of thermal power and social welfare. 


\section{Chapter 4}

\section{An Options Pricing Approach to Ramping Rate Restrictions at Hydro Power Plants: An Extension to Three Price Regimes}

\subsection{Introduction}

In the third chapter (Niu and Insley [2014]), the empirical study on the impact of ramping restrictions on our prototype plant is based on the Markov regime switching model of Janczura and Weron [2009], where we assume a constant up-jump size when the price process switches from the base regime to the spike regime and a constant down-jump size when the price process switches from the spike regime to the base regime. The up-jump size is set equal to the ratio of the spike regime mean price to the base regime mean price and the down-jump size is roughly the inverse of the up-jump size. However, when the regime switching occurs it is highly unlikely that the price will jump up or down at some constant size. In fact, the fixed jump size used in the third chapter only represents the average size of a jump when the regime switching happens. Therefore, it is desirable to 
extend the Markov regime switching model of Janczura and Weron [2009] to a model with multiple jump sizes, which allows us to examine the impact of a random jump size on our results.

In this chapter, we conduct numerical experiments on the jump size by proposing a regime switching model with three regimes: the base regime (regime 1), the first spike regime (regime 2), and the second spike regime (regime 3). Assuming different jump sizes among these three regimes allows us to study the effect of multiple jump sizes on the impact of ramping restrictions on the hydro plant. Ideally, we could use more regimes to approximate the random jump size as close as possible. However, this will significantly increase the computational complexity for this regime switching model. In fact, different jump sizes among these three regimes in our proposed model could be viewed as the simplest approximation of the random jump size observed from the data. To reduce the computational burden, we further assume that only two possible switches among these three regimes are permitted: switching between regimes 1 and 2; switching between regimes 1 and 3. In the empirical analysis, the jump size between the base regime and the first spike regime is the same as the jump size (which represents the average jump size) between the base regime and the spike regime in chapter 3. We will analyze two cases for the jump size between the base regime and the second spike regime: a larger than the average jump size; a smaller than the average jump size.

The empirical results from this chapter show that, even if multiple jump sizes are incorporated into the regime switching model by adding the third regime, the findings are very similar to what we get in the third chapter. There is a significant effect on profits for the most restrictive ramping constraints, but we also observe a range of ramping restrictions over which profits are not substantially affected. These numerical experiments provide further evidence that ramping restrictions have a larger impact when the expected variation in price is increased such as through an increase in jump size (for the third regime) which makes it desirable to change water release rates relatively frequently.

The remainder of the chapter is organized as follows. In the next section we present the model and associated parameter values used in the empirical analysis. Sections three and four contain the results from two numerical experiments: large jump size from the base 
regime to the second spike regime; small jump size from the base regime to the second spike regime. Section five concludes.

\subsection{Empirical Analysis}

The empirical analysis in this study is based on the medium-sized hydro plant similar to the Abitibi Canyon generating station located on the Abitibi River in northeastern Ontario. Detailed descriptions about this hydro plant are provided in chapter two. The input parameters for the hydro power production and hydro dam specifications are reported in Table 3.1 of chapter three. Since adding another regime to the model will significantly increase the computation time, the analysis is conducted for a time horizon of three days 1 .

Similar to the third chapter, we base our regime switching model on the model estimated in Janczura and Weron [2009]. Under the Q measure, it is assumed the regime switching process can be specified as follows for the base, the first spike, and the second spike regimes respectively:

$$
\begin{gathered}
d P=\left[\eta\left(\mu_{1}-P\right)-\Lambda_{1} \sigma_{1} \sqrt{P}\right] d t+\sigma_{1} \sqrt{P} d \hat{Z}+P\left(\xi^{12}-1\right) d \hat{X}_{12}+P\left(\xi^{13}-1\right) d \hat{X}_{13} . \\
d P=\sigma_{2}(P-m) d \hat{z}+P\left(\xi^{21}-1\right) d \hat{X}_{21}, P>m . \\
d P=\sigma_{3}(P-m) d \check{z}+P\left(\xi^{31}-1\right) d \hat{X}_{31}, P>m .
\end{gathered}
$$

where $d \hat{Z}, d \hat{z}$, and $d \check{z}$ are the increments of the standard Gauss-Wiener processes under the $\mathrm{Q}$ measure specified by the choice of $\Lambda_{1}, \Lambda_{2}$, and $\Lambda_{3}$ respectively. $d \hat{X}_{12}, d \hat{X}_{21}, d \hat{X}_{13}$, and $d \hat{X}_{31}$ indicate the transition of the Markov chain under the $\mathrm{Q}$ measure.

The parameter values used for Equations (4.1), (4.2) and (4.3) are shown in Table 4.1. The long run price, $\mu_{1}$, median price, $m$, mean reversion speed, $\eta$, and volatilities $\sigma_{1}$ and

\footnotetext{
${ }^{1}$ In the empirical experiments, the computational time for each case varies from several days to two weeks.
} 
Table 4.1: Parameter Values for the Regime Switching Model (Three Price Regimes)

\begin{tabular}{cccccc}
\hline Parameter & Value & Parameter & Value & Parameter & Value \\
\hline$\mu_{1}$ & 47.194 EUR/MWh & $\eta$ & 0.36 & - & - \\
$m$ & 46.54 EUR/MWh & $c$ & 20 EUR/MWh & - & - \\
$\sigma_{1}$ & 0.73485 & $\sigma_{2}$ & 0.83066 & $\sigma_{3}$ & 0.83066 \\
$\xi^{12}$ & 1.6470 & $\xi^{21}$ & 0.6072 & - & - \\
$\xi^{13}$ & $1.8470(1.4470)$ & $\xi^{31}$ & $0.6911(0.5414)$ & - & - \\
$\lambda_{11}^{Q}$ & 0.991 & $\lambda_{12}^{Q}$ & 0.0045 & $\lambda_{13}^{Q}$ & 0.0045 \\
$\lambda_{21}^{Q}$ & 0.8402 & $\lambda_{22}^{Q}$ & 0.1598 & $\lambda_{23}^{Q}$ & 0 \\
$\lambda_{31}^{Q}$ & 0.8402 & $\lambda_{32}^{Q}$ & 0 & $\lambda_{33}^{Q}$ & 0.1598 \\
$\Lambda_{1}^{Q}$ & -0.2481 & $\Lambda_{2}$ & -0.2481 & $\Lambda_{3}$ & -0.2481 \\
$P_{1}^{\max }$ & $200 \mathrm{EUR} / \mathrm{MWh}$ & $P_{2}^{\max }$ & $200 \mathrm{EUR} / \mathrm{MWh}$ & $P_{3}^{\max }$ & $200 \mathrm{EUR} / \mathrm{MWh}$ \\
$P_{1}^{\min }$ & $0 \mathrm{EUR} / \mathrm{MWh}$ & $P_{2}^{\min }$ & $48 \mathrm{EUR} / \mathrm{MWh}$ & $P_{3}^{\min }$ & $48 \mathrm{EUR} / \mathrm{MWh}$ \\
$T$ & $72 \mathrm{~h}$ & $\bar{r}$ & $0.05 \mathrm{annually}$ & - & - \\
\hline
\end{tabular}

Base regime: $d P=\left[\eta\left(\mu_{1}-P\right)-\Lambda_{1} \sigma_{1} \sqrt{P}\right] d t+\sigma_{1} \sqrt{P} d \hat{Z}+P\left(\xi^{12}-1\right) d \hat{X}_{12}+P\left(\xi^{13}-1\right) d \hat{X}_{13}$. First spike regime: $d P=\sigma_{2}(P-m) d \hat{z}+P\left(\xi^{21}-1\right) d \hat{X}_{21}, P>m$.

Second spike regime: $d P=\sigma_{3}(P-m) d \check{z}+P\left(\xi^{31}-1\right) d \hat{X}_{31}, P>m$.

$\sigma_{2}$ are taken from the Janczura and Weron estimates. The volatility for the second spike regime $\sigma_{3}$ is set equal to $\sigma_{2}$. The up-jump and down-jump sizes between the base and the first spike regimes $\xi^{12}$ and $\xi^{21}$ are the same as the jump sizes between the base and spike regimes in chapter 3. We examine two cases for the jump size from the base regime to the second spike regime $\xi^{13}$ : a larger jump size than $\xi^{12}$ (the average jump size); a smaller jump size (the value in the bracket) than $\xi^{12}$. $\xi^{31}$ refers to the corresponding down-jump size. $\lambda_{\imath \jmath}^{Q}, \imath, \jmath=1,2,3$ represent the regime transition intensities in the risk neutral world. $\lambda_{12}^{Q}$ is set equal to $\lambda_{13}^{Q}, \lambda_{21}^{Q}$ is set equal to $\lambda_{31}^{Q}$, and $\lambda_{22}^{Q}$ is set equal to $\lambda_{33}^{Q}$. The transition intensities between the first and second spike regimes $\lambda_{23}^{Q}$ and $\lambda_{32}^{Q}$ are zero since the transition between these two regimes is not permitted by assumption. The market price of risk $\Lambda$ is taken from Cartea and Figueroa [2005] and assumed the same for the three regimes. Table 4.1 also reports the assumed variable cost of electricity production, $c$, the upper and lower limits on price in the numerical analysis, $P_{\imath}^{\min }$ and $P_{\imath}^{\max }, \imath=1,2,3$, the timeframe for the analysis in hours, $T$, and the risk free rate, $\bar{r}$. 


\subsection{Case I: Large Jump Size from the Base Regime to the Second Spike Regime}

For Case I, we examine in detail the impact of ramping restrictions on our prototype power plant for a large jump size from the base regime to the second spike regime. In this case, Table 4.1 shows that the up-jump size $\xi^{13}$ is 1.8470 and the down-jump size $\xi^{31}$ is 0.6911 .

\subsubsection{Base Case for Case I}

In this section, we present the numerical results for the base case when both the up-ramping and down-ramping constraints are set at 3,000 CFS-hr. Similar to the third chapter, we will report the numerical results for the following relationships: power plant value versus price and release rate; optimal ramping rate versus price and release rate; optimal ramping rate versus price and time; power plant value versus price and reservoir level; optimal ramping rate versus price and reservoir level.

Figures 4.1(a) through 4.5(c) show the results for this base case ${ }^{2}$ when the constraints for ramping rate, water release rate and water content are imposed. We first focus on the value of the hydro plant as a function of water release rate and spot price. In Figures 4.1(b) and 4.1(c) the first and second spike regimes are defined for prices from 46.54 to 200 EUR/MWh. However, in Figure 4.1(a) the base regime is defined for the domain from 0 to $200 \mathrm{EUR} / \mathrm{MWh}$. In the base regime we find that the value of the hydro plant and the spot price are positively correlated. However, the hydro plant's value and the water release rate are positively correlated if the price is higher than $20 \mathrm{EUR} / \mathrm{MWh}$ and negatively correlated if the price is lower than $20 \mathrm{EUR} / \mathrm{MWh}$. When the spot price is in one of the two spike regimes the value of the hydro plant is positively correlated with both water release rate and spot price. As expected, at any given water release rate and spot price, the value of the hydro plant in the second spike regime is higher than its value in the first spike regime and the hydro plant has a higher value in both spike regimes than in the base regime.

\footnotetext{
${ }^{2}$ Figures 4.1(a) through 4.5(c) in this chapter are corresponding to Figures 3.3(a) through 3.7(b) in chapter three, but include two spike regimes.
} 
Specifically, in the base regime the hydro plant has the maximum value of EUR 813,250 at the release rate of 15,000 CFS and the price of $200 \mathrm{EUR} / \mathrm{MWh}$ and the minimum value of EUR 567,090 at the release rate of 15,000 CFS and the price of 0 EUR/MWh. In addition, in the first spike regime (the second spike regime) the hydro plant has the maximum value of EUR 853,760 (EUR 880,130) at the release rate of 15,000 CFS and the price of 200 EUR/MWh and the minimum value of EUR 610,040 (EUR 613,080) at the release rate of 2,000 CFS and the price of 48 EUR/MWh.

We plot the optimal control strategies in Figures 4.2(a), 4.2(b), and 4.2(c), which are corresponding to Figures 4.1(a), 4.1(b), and 4.1(c) respectively. Generally, for the base regime the power plant will ramp up (ramp down) at the maximum limit of 3,000 CFS-hr if the price is high (low). Similar to our findings in the third chapter, the constraints on release rates and reservoir size also have an impact on optimal operations particularly in the maximum and minimum release rate regions. For example, Figure 4.2(a) shows in the low price and low release rate region the power plant must ramp up to avoid violating the minimum release constraint. However as the release rate is increased, but remaining at low prices the optimal action switches from ramping up to ramping down. Next, in the high release rate and high price region the operator is not allowed to ramp up at the maximum rate due to the maximum release rate constraint. The optimal control strategies for the two spike regimes are virtually the same. Because of the much higher price in the spike regime it is optimal for the hydro station to ramp up at 3,000 CFS-hr whenever possible given the other constraints.

Regarding the optimal operational strategies for the hydro power plant as a function of time and spot price, for the base regime Figures 4.3(a) shows that at low prices it is optimal to ramp down and for high prices it is optimal to ramp up. The optimal control strategy remains largely unchanged over time. At any given hour (except the terminal time region) we observe four operational regions: ramping down at the maximum limit of 3,000 CFS-hr if the price is below $20 \mathrm{EUR} / \mathrm{MWh}$; ramping down at 2,000 CFS-hr if the price lies between $20 \mathrm{EUR} / \mathrm{MWh}$ and $40 \mathrm{EUR} / \mathrm{MWh}$; ramping down at 1,500 CFS-hr when the price is between $40 \mathrm{EUR} / \mathrm{MWh}$ and $60 \mathrm{EUR} / \mathrm{MWh}$; ramping up at the maximum limit of 3,000 CFS-hr as the price moves above $60 \mathrm{EUR} / \mathrm{MWh}$. Interestingly, the optimal control 
strategy changes dramatically in the terminal time region. Given the terminal boundary condition of zero value, it is optimal to ramp up at the maximum allowed rate as time moves into the terminal time region. For the two spike regimes (Figures 4.3(b) and 4.3(c)) higher prices make the station mostly ramp up at the maximum limit of 3,000 CFS-hr.

Now, we analyze the results for the value of the hydro power plant as a function of reservoir level and spot price. In all the three regimes (Figures 4.4(a), 4.4(b), and 4.4(c)) we find that the value of the hydro power plant has positive relationships with both the reservoir level and spot price. Not surprisingly, at any given reservoir level and spot price, the value of the hydro plant in the second spike regime is higher than its value in the first spike regime and the base regime has the lowest value among these three regimes. Specifically, in the base regime the power plant reaches the highest value of EUR 787,790 at the reservoir level of 17,000 acre-feet and the price of $200 \mathrm{EUR} / \mathrm{MWh}$ and the lowest value of EUR 471,260 at the reservoir level of 7,000 acre-feet and the price of $0 \mathrm{EUR} / \mathrm{MWh}$. In addition, in the first spike regime (the second spike regime) the power plant reaches the highest values of EUR 826,490 (EUR 851,360) at the reservoir level of 17,000 acre-feet and the price of $200 \mathrm{EUR} / \mathrm{MWh}$ and the lowest value of EUR 495,950 (EUR 498,720) at the reservoir level of 7,000 acre-feet and the price of $48 \mathrm{EUR} / \mathrm{MWh}$.

The optimal control strategies corresponding to Figures 4.4(a), 4.4(b), and 4.4(c) are shown in Figures 4.5(a), 4.5(b), and 4.5(c) respectively. As usual, in the base regime it is optimal to ramp up at the maximum limit of 3,000 CFS-hr at high prices and ramp down at the maximum limit of 3,000 CFS-hr at low prices. However, in the low price region the power plant operates quite differently when the reservoir level is close to or at the minimum or maximum limit. First, in the low price and low reservoir level region the power plant will ramp up at the maximum limit of 3,000 CFS-hr, but this tends to violate the lower water content constraint. ${ }^{3}$ However, this should not cause any concerns because it is not optimal for the power plant to operate anywhere close to the lower reservoir boundary. Second, in the low price and full reservoir region the power plant will ramp down at rates lower than the maximum allowed rate to keep the reservoir full. In addition, we also observe that there

\footnotetext{
${ }^{3}$ These results are obtained for the short time horizon of three days. If the model is run for a longer time horizon (seven days), we should obtain more stable results similar to our findings in the third chapter.
} 
are some critical prices and reservoir levels where there is an operational boundary between up ramping at the maximum limit of 3,000 CFS-hr and down ramping at the maximum limit of 3,000 CFS-hr. For the two spike regimes, the power plant operates similarly to the base regime when the price is higher than $48 \mathrm{EUR} / \mathrm{MWh}$.

\subsubsection{Impact of Changing Ramping Restrictions for Case I}

For the hydro power plant, the numerical results for the impact of ramping constraints on profits are summarized in Table 4.2, which is plotted in Figure 4.6(a) for the case of half release rate and full reservoir level and in Figure 4.6(b) for the case of full release rate and full reservoir level. Similar to what we find in the third chapter, as the constraints become more restrictive the impact on profits becomes larger. However, these two figures also show that for a large range of ramping rate restrictions the profit is not very sensitive to these restrictions. Assuming everything else is held constant, higher initial electricity price is always associated with higher present value. Given the same initial price, the second spike regime (with a large jump size from the base regime to the spike regime) always gives higher present value than the first spike regime (with an average jump size from the base regime to the spike regime).

Given the initial price at $40 \mathrm{EUR} / \mathrm{MWh}$, the reservoir level at the full capacity, the water release rate at the upper limit and the inflow rate at 6,671 CFS, with ramping constraints of $250 \mathrm{CSF}-\mathrm{hr}$ the total profit drops $7.9 \%$ relative to the scenario without ramping restrictions. The reduction of profit in this most restrictive scenario is very similar to what is found in chapter 3 where the total profit is reduced by $8.3 \%$ under the most severe ramping restrictions. If the initial release rate is half of the upper limit while keeping other conditions the same, then the total profit only drops $5.8 \%$ relative to the scenario without ramping restrictions. Figures 4.6(a) and 4.6(b) show that profits are becoming more sensitive for the restrictions on ramping rates of less than 1,000 CFS-hr and 3,000 CFS-hr respectively. We obtain similar results for different initial price, water release rate and regime. When the price jumps to $160 \mathrm{EUR} / \mathrm{MWh}$ in the spike regime, the total profit only drops $8.7 \%$ at the most restrictive ramping constraints of $250 \mathrm{CSF}-\mathrm{hr}$. 


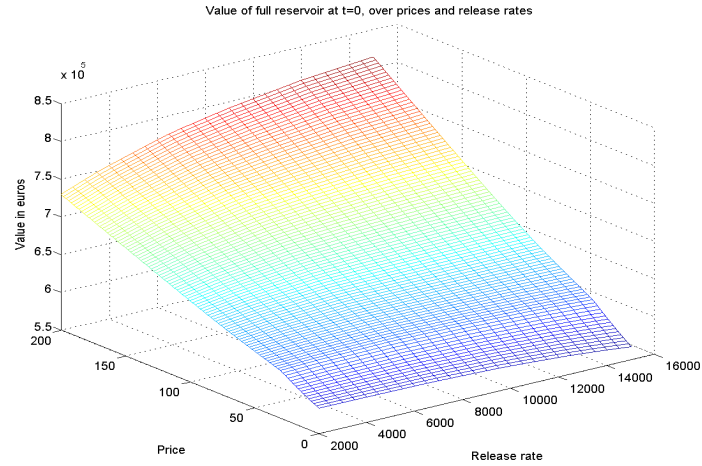

(a) Value over Price and Release Rate for the Base Regime

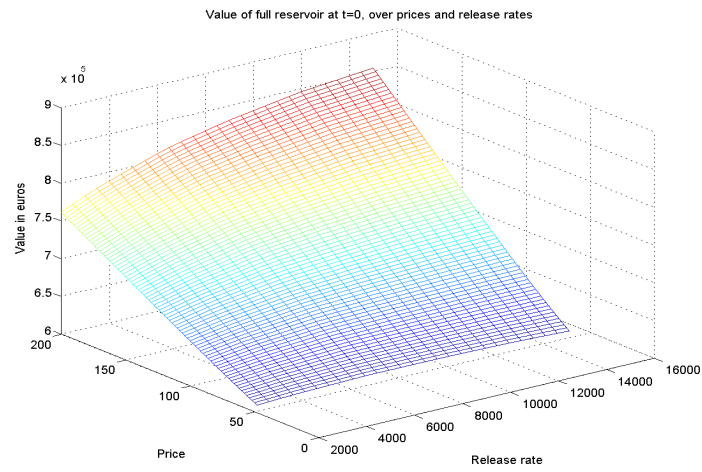

(b) Value over Price and Release Rate for the First Spike Regime

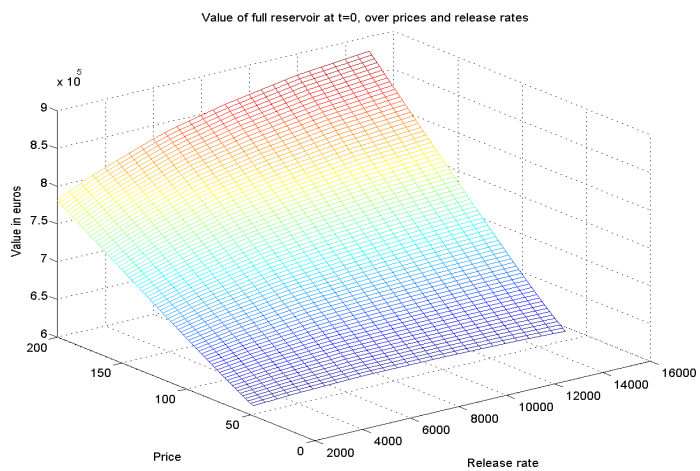

(c) Value over Price and Release Rate for the Second Spike Regime

Figure 4.1: Value over Price and Release Rate for the Base, the First Spike, and the Second Spike Regimes (Case I) 


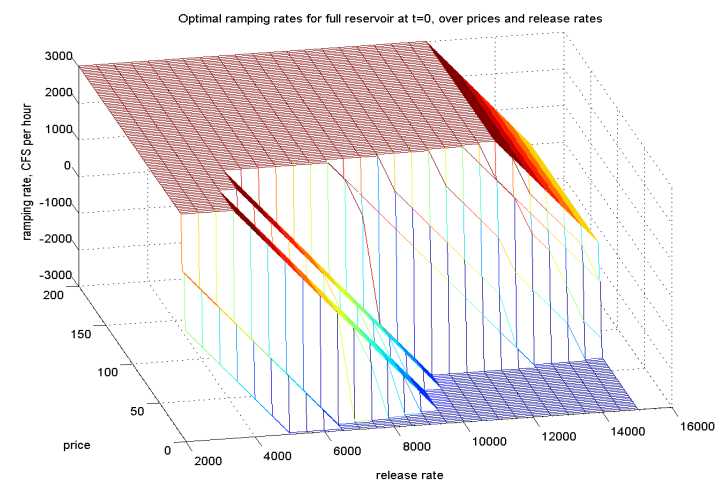

(a) Optimal Ramping Rate over Price and Release Rate for the Base Regime

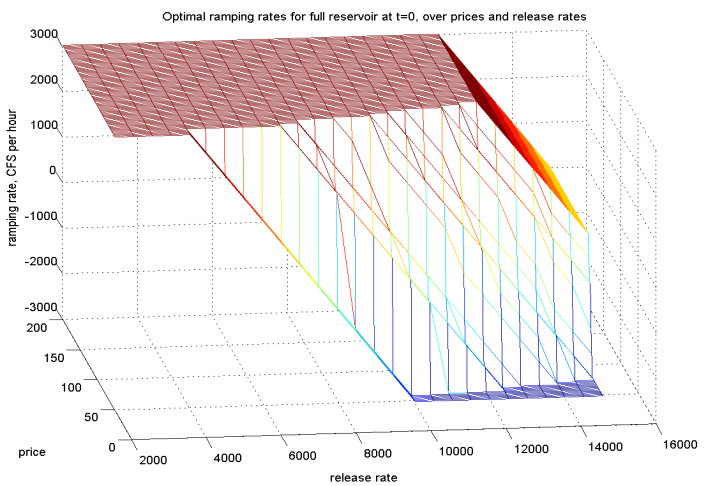

(b) Optimal Ramping Rate over Price and Release Rate for the First Spike Regime

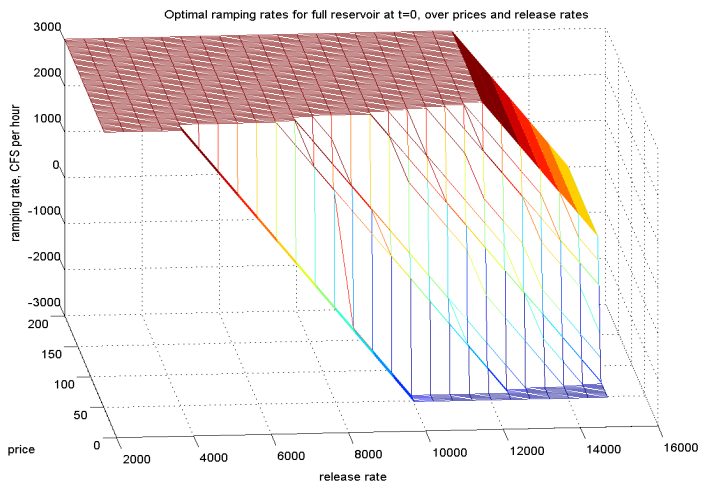

(c) Optimal Ramping Rate over Price and Release Rate for the Second Spike Regime

Figure 4.2: Optimal Ramping Rate over Price and Release Rate for the Base, the First Spike, and the Second Spike Regimes (Case I) 


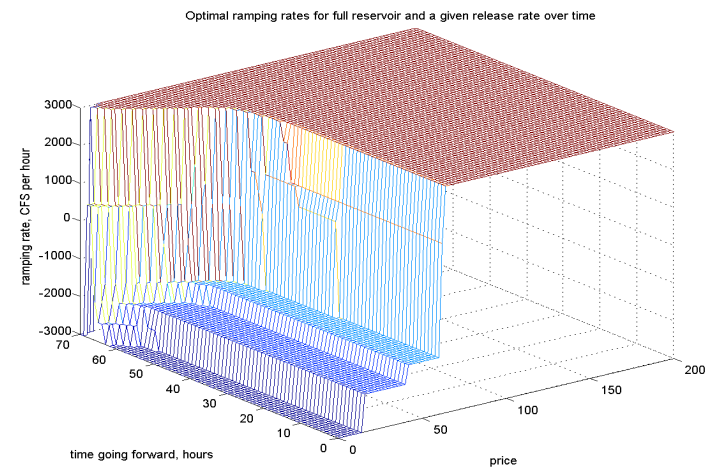

(a) Optimal Ramping Rate over Price and Time for the Base Regime

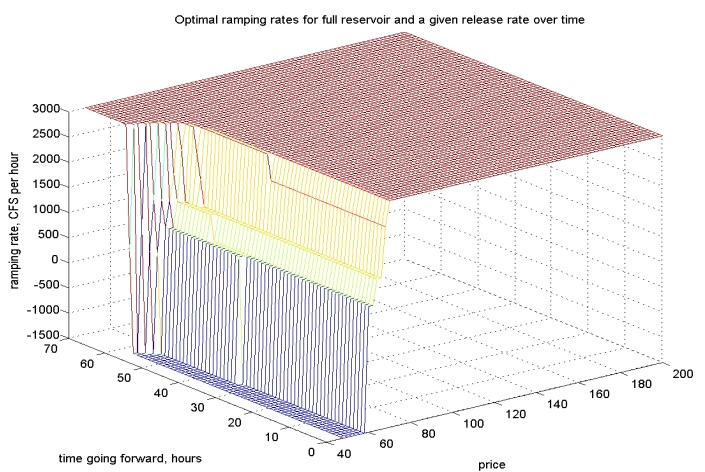

(b) Optimal Ramping Rate over Price and Time for the First Spike Regime

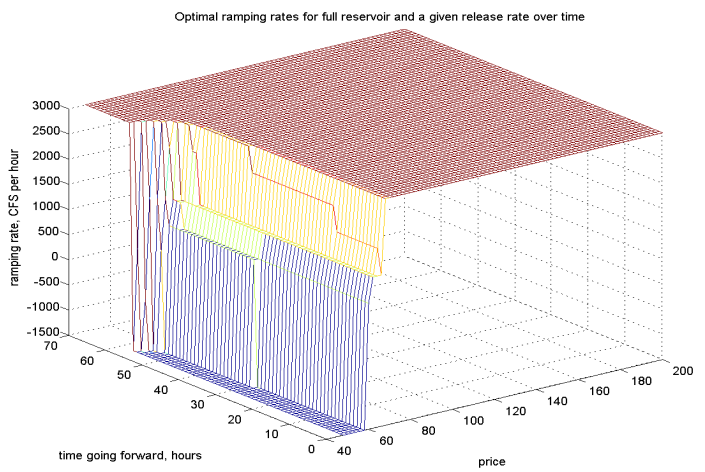

(c) Optimal Ramping Rate over Price and Time for the Second Spike Regime

Figure 4.3: Optimal Ramping Rate over Price and Time for the Base, the First Spike, and the Second Spike Regimes (Case I) 


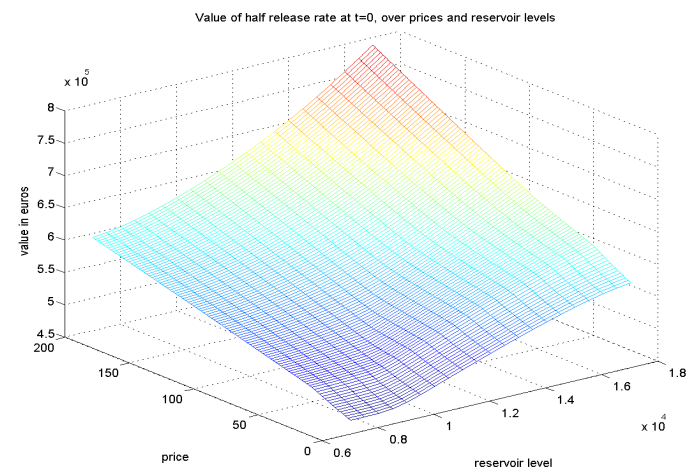

(a) Value over Price and Reservoir Level for the Base Regime

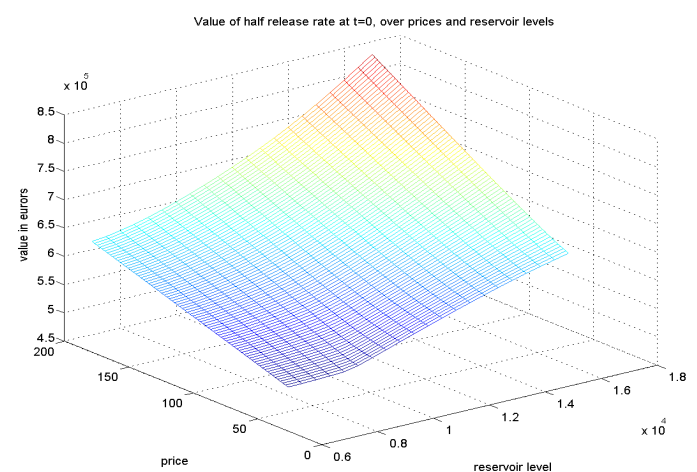

(b) Value over Price and Reservoir Level for the First Spike Regime

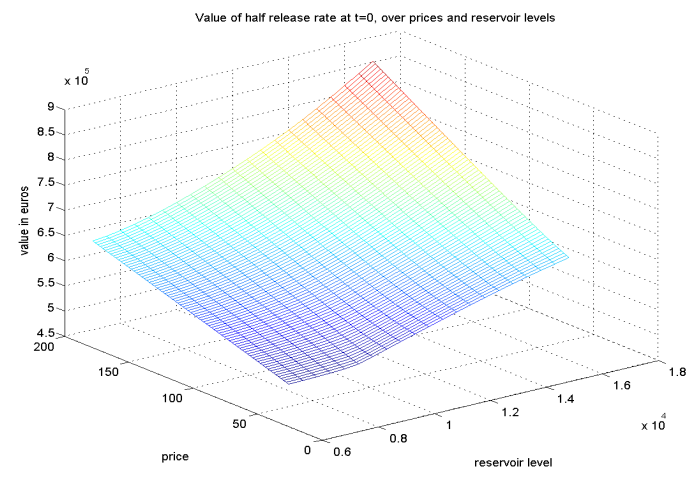

(c) Value over Price and Reservoir Level for the Second Spike Regime

Figure 4.4: Value over Price and Reservoir Level for the Base, the First Spike, and the Second Spike Regimes (Case I) 


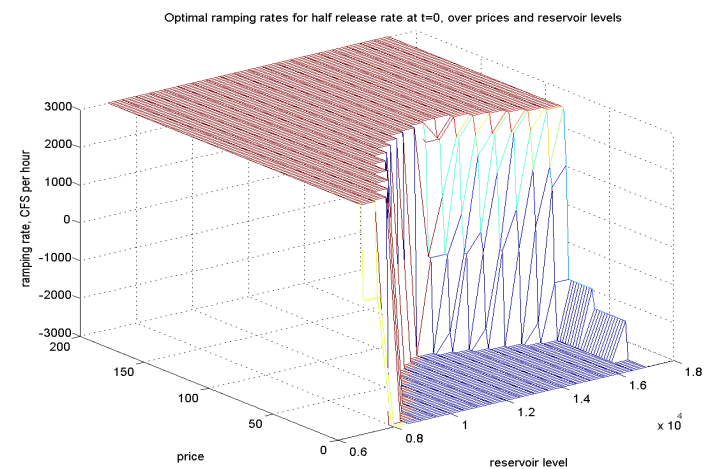

(a) Optimal Ramping Rate over Price and Reservoir Level for the Base Regime

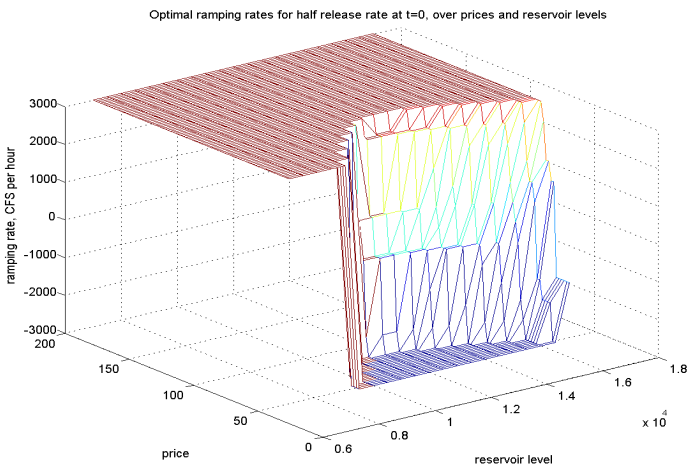

(b) Optimal Ramping Rate over Price and Reservoir Level for the First Spike Regime

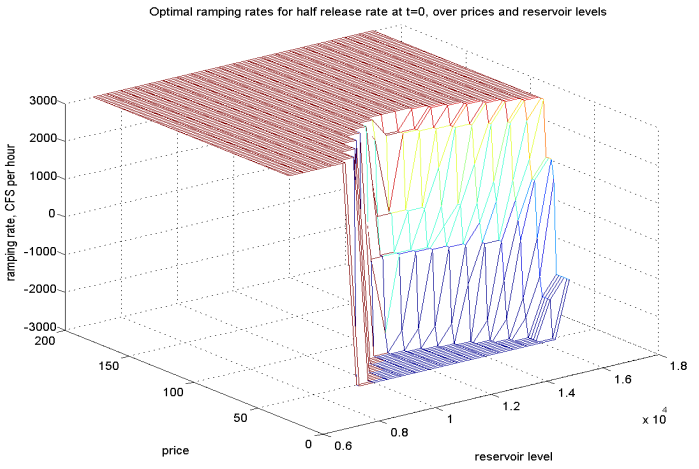

(c) Optimal Ramping Rate over Price and Reservoir Level for the Second Spike Regime

Figure 4.5: Optimal Ramping Rate over Price and Reservoir Level for the Base, the First Spike, and the Second Spike Regimes (Case I) 106 
Table 4.2: Numerical Results Under Various Ramping Restrictions (Large Jump Size from the Base Regime to the Second Spike Regime)

\begin{tabular}{|c|c|c|c|c|c|c|}
\hline Case & & $\begin{array}{l}\text { No Ramping } \\
\text { Restrictions }\end{array}$ & 5000 (CFS-hr) & 3000 (CFS-hr) & 1000 (CFS-hr) & 250 (CFS-hr) \\
\hline \multirow[t]{2}{*}{$\mathrm{HF}$} & Profit & 629520 & 627370 & 623880 & 616160 & 592880 \\
\hline & $\%$ ch & $\mathrm{N} / \mathrm{A}$ & -0.3 & -0.9 & -2.1 & -5.8 \\
\hline \multirow[t]{2}{*}{ FF } & Profit & 628190 & 624780 & 618940 & 594120 & 578810 \\
\hline & $\%$ ch & $\mathrm{N} / \mathrm{A}$ & -0.5 & -1.5 & -5.4 & -7.9 \\
\hline \multicolumn{7}{|c|}{ Total Profit and Change of Total Profit in Regime 2 at Time 0 When the Initial Price is $80 \mathrm{EUR} / \mathrm{MWh}$} \\
\hline \multirow[t]{2}{*}{$\mathrm{HF}$} & Profit & 662050 & 659080 & 654610 & 644370 & 619800 \\
\hline & $\%$ ch & $\mathrm{N} / \mathrm{A}$ & -0.5 & -1.1 & -2.7 & -6.4 \\
\hline \multirow[t]{2}{*}{$\mathrm{FF}$} & Profit & 664670 & 661570 & 656540 & 635530 & 623470 \\
\hline & $\% \mathrm{ch}$ & $\mathrm{N} / \mathrm{A}$ & -0.5 & -1.2 & -4.4 & -6.2 \\
\hline \multicolumn{7}{|c|}{ "Total Profit and Change of Total Profit in Regime 2 at Time 0 When the Initial Price is 160 EUR/MWh } \\
\hline \multirow{2}{*}{ HF } & Profit & 779940 & 775230 & 768060 & 747290 & 714710 \\
\hline & $\%$ ch & N/A & -0.6 & -1.5 & -4.2 & -8.4 \\
\hline \multirow[t]{2}{*}{ FF } & Profit & 792790 & 790360 & 786780 & 776460 & 773980 \\
\hline & $\% \mathrm{ch}$ & $\mathrm{N} / \mathrm{A}$ & -0.3 & -0.8 & -2.1 & -2.4 \\
\hline \multicolumn{7}{|c|}{ Total Profit and Change of Total Profit in Regime 3 at Time 0 When the Initial Price is $80 \mathrm{EUR} / \mathrm{MWh}$} \\
\hline \multirow[t]{2}{*}{$\mathrm{HF}$} & Profit & 668970 & 666000 & 661500 & 650980 & 626200 \\
\hline & $\%$ ch & N/A & -0.4 & -1.1 & -2.7 & -6.4 \\
\hline \multirow[t]{2}{*}{ FF } & Profit & 671570 & 668640 & 663900 & 644330 & 633720 \\
\hline & $\%$ ch & $\mathrm{N} / \mathrm{A}$ & -0.4 & -1.1 & -4.1 & -5.6 \\
\hline \multicolumn{7}{|c|}{ Total Profit and Change of Total Profit in Regime 3 at Time 0 When the Initial Price is $160 \mathrm{EUR} / \mathrm{MWh}$} \\
\hline \multirow[t]{2}{*}{$\mathrm{HF}$} & Profit & 799210 & 794340 & 786920 & 764430 & 729630 \\
\hline & $\% \mathrm{ch}$ & $\mathrm{N} / \mathrm{A}$ & -0.6 & -1.5 & -4.4 & -8.7 \\
\hline \multirow[t]{2}{*}{$\mathrm{FF}$} & Profit & 812460 & 810120 & 806730 & 797610 & 795770 \\
\hline & $\% \mathrm{ch}$ & $\mathrm{N} / \mathrm{A}$ & -0.3 & -0.7 & -1.8 & -2.1 \\
\hline
\end{tabular}

Note: HF means half release rate and full reservoir level; FF means full release rate and full reservoir level. Profit is in Euros and \%ch refers to the percent change in profits compared to the case of no ramping restrictions. 


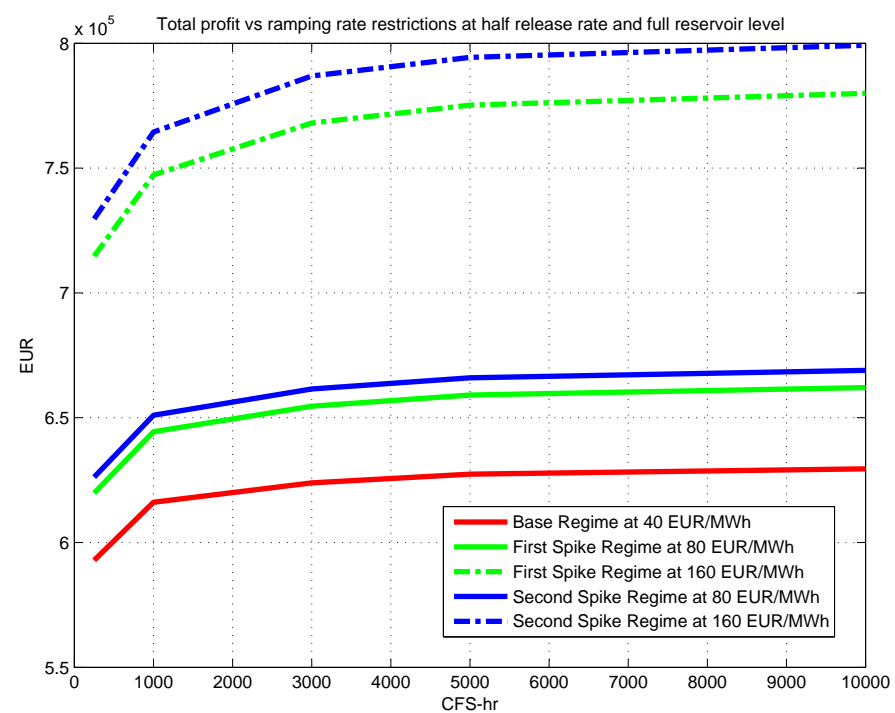

(a) Total Profit vs Ramping Rate Restrictions at Half Release Rate and Full Reservoir Level

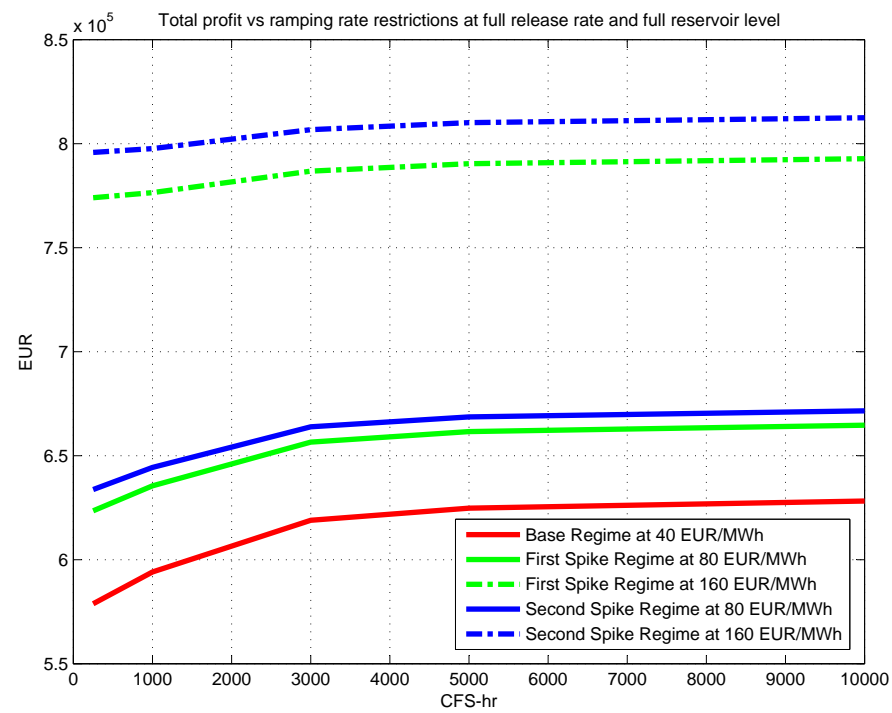

(b) Total Profit vs Ramping Rate Restrictions at Full Release Rate and Full Reservoir Level

Figure 4.6: Total Profit vs Ramping Rate Restrictions at Half Release Rate and Full Reservoir Level and at Full Release Rate and Full Reservoir Level (Case I) 


\subsection{Case II: Small Jump Size from the Base Regime to the Second Spike Regime}

In this section, we investigate the impact of ramping restrictions on our prototype power plant for a small jump size from the base regime to the second spike regime. Now, the up-jump size $\xi^{13}$ is changed to 1.4470 and the down-jump size $\xi^{31}$ is set at 0.5414 (Table 4.1). We first report the numerical results for the base case with both the up-ramping and down-ramping constraints at 3,000 CFS-hr and then analyze the impact of the ramping constraints on the total profit of the hydro plant over a range of ramping constraints. Since the results for this small jump size case are very similar to the findings for the large jump size case, we will mainly focus on their differences.

It is noticed that Figures 4.7(a) through 4.11(c) for Case II are corresponding to Figures 4.1(a) through 4.5(c) for Case I. First, Figures 4.7(a), 4.7(b), and 4.7(c) show how the value of the hydro plant varies with the water release rate and spot price ${ }^{4}$. As expected, at any given water release rate and spot price, the hydro plant has a higher value in both spike regimes than in the base regime, but the value of the hydro plant in the second spike regime is lower than its value in the first spike regime. Specifically, in the base regime the power plant reaches the highest value of EUR 801,480 at the release rate of 15,000 CFS and the price of $200 \mathrm{EUR} / \mathrm{MWh}$ and the lowest value of EUR 552,940 at the release rate of 15,000 CFS and the price of 0 EUR/MWh. Furthermore,, in the first spike regime (the second spike regime) the power plant has the maximum value of EUR 842,130 (EUR 821,560) at the release rate of $15,000 \mathrm{CFS}$ and the price of $200 \mathrm{EUR} / \mathrm{MWh}$ and the minimum value of EUR 596,020 (EUR 592,050) at the release rate of 2,000 CFS and the price of 48 EUR/MWh.

Figures 4.10(a), 4.10(b), and 4.10(c) shows how the value of the hydro power plant depends on the reservoir level and spot price $^{5}$. Not surprisingly, at any given reservoir level

\footnotetext{
${ }^{4}$ The corresponding optimal control strategies in Figures 4.8(a), 4.8(b), and 4.8(c) are virtually the same as those shown in Figures 4.2(a), 4.2(b), and 4.2(c) for Case I. In addition, in Figures 4.9(a), 4.9(b), and $4.9(\mathrm{c})$ the optimal operational strategies for the hydro power plant as a function of time and spot price are also very similar to those shown in Figures 4.3(a), 4.3(b), and 4.3(c) for Case I.

${ }^{5}$ The associated optimal control strategies in Figures 4.11(a), 4.11(b), and 4.11(c) behave similarly as
} 
and spot price, the value of the hydro plant in the first spike regime is higher than its value in the second spike regime and the base regime gives the lowest value. In particular, in the base regime the power plant has the highest value of EUR 775,520 when the current reservoir level is 17,000 acre-feet and the price is $200 \mathrm{EUR} / \mathrm{MWh}$ and the lowest value of EUR 461,040 when the current reservoir level is 7,000 acre-feet and the price is $0 \mathrm{EUR} / \mathrm{MWh}$. Additionally, in the first spike regime (the second spike regime) the value is at the highest level of EUR 814,360 (EUR 794,960) when the current reservoir level is 17,000 acre-feet and the price is $200 \mathrm{EUR} / \mathrm{MWh}$ and at the lowest level of EUR 486,030 (EUR 482,550) when the current reservoir level is 7,000 acre-feet and the price is $48 \mathrm{EUR} / \mathrm{MWh}$.

Now, we analyze impact of the ramping constraints on the value of the hydro power plant. The results are reported in Table 4.3 and plotted in Figures 4.12(a) and 4.12(b). Similar to what we find for Case I, more restrictive constraints have a larger impact on profits. But, the profit is not very sensitive to ramping rate restrictions. As usual, if we keep everything else constant, higher initial electricity price always results in higher present value. However, for the case of small jump size from the base regime to the second spike regime, given the same initial price the second spike regime (with a small jump size from the base regime to the spike regime) always gives lower present value than the first spike regime (with an average jump size from the base regime to the spike regime). As expected, the small jump size case gives an overall lower value than the large jump size case. In addition, in the small jump size case ramping restrictions have a smaller impact than in the large jump size case. However the change is again quite small, with the most severe restrictions reducing profits by $8.2 \%$ in the small jump size case compared to $8.7 \%$ in the large jump size case. Restrictive ramping constraints will limit the power plant's ability to make profits during price spikes, therefore the power plant's value is more severely affected when the spike regime has a large jump size.

those shown in Figures 4.5(a), 4.5(b), and 4.5(c) for Case I. 


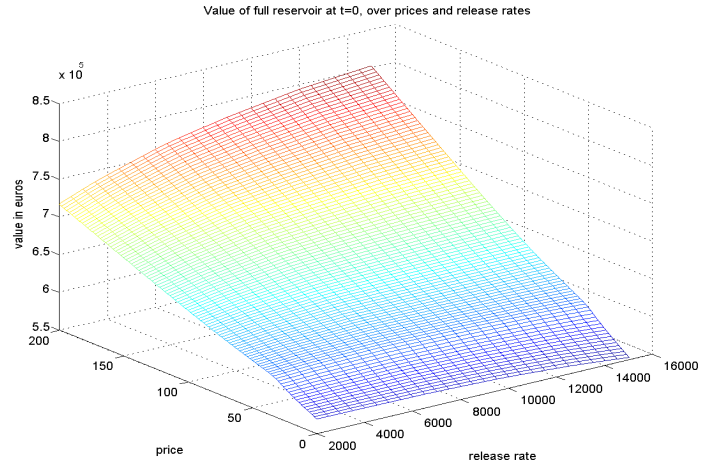

(a) Value over Price and Release Rate for the Base Regime

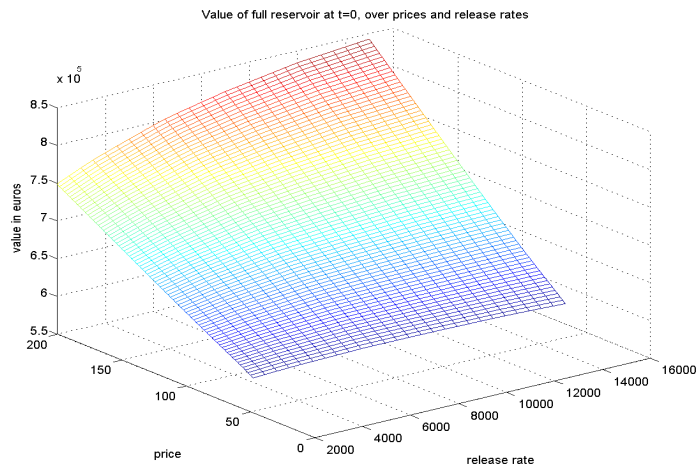

(b) Value over Price and Release Rate for the First Spike Regime

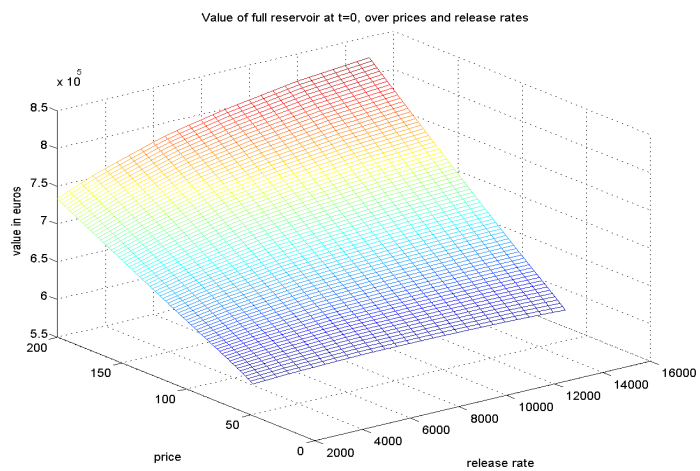

(c) Value over Price and Release Rate for the Second Spike Regime

Figure 4.7: Value over Price and Release Rate for the Base, the First Spike, and the Second Spike Regimes (Case II) 


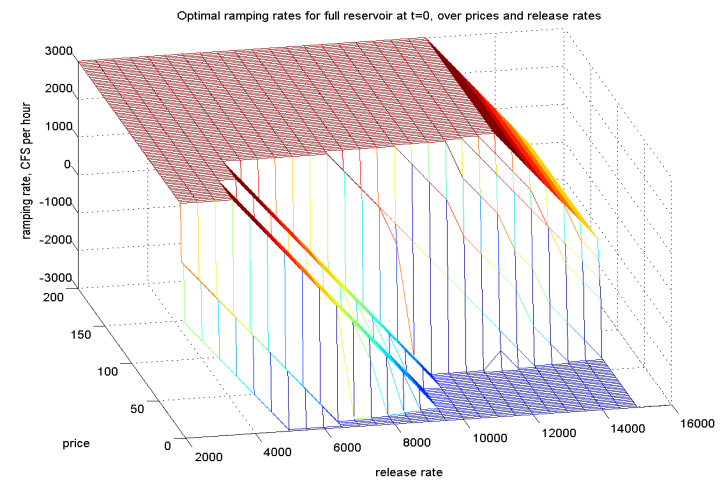

(a) Optimal Ramping Rate over Price and Release Rate for the Base Regime

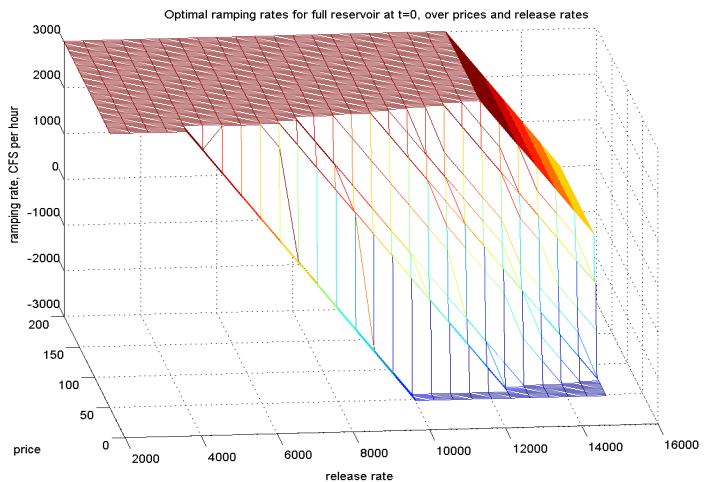

(b) Optimal Ramping Rate over Price and Release Rate for the First Spike Regime

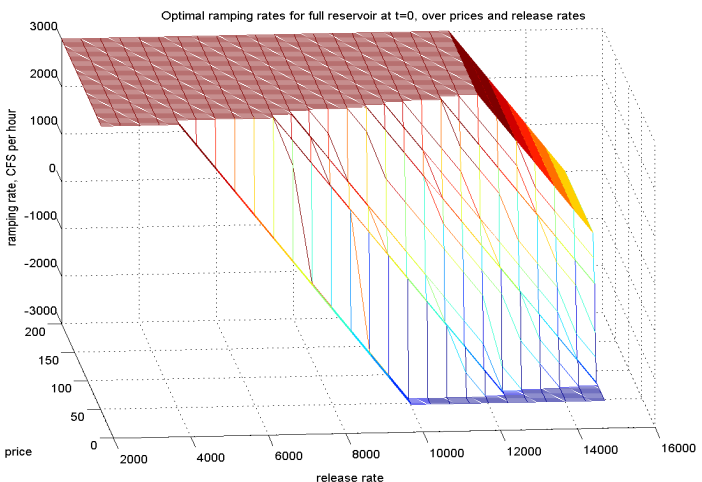

(c) Optimal Ramping Rate over Price and Release Rate for the Second Spike Regime

Figure 4.8: Optimal Ramping Rate over Price and Release Rate for the Base, the First Spike, and the Second Spike Regimes (Case $\left.11 \frac{1}{2}\right)$ 


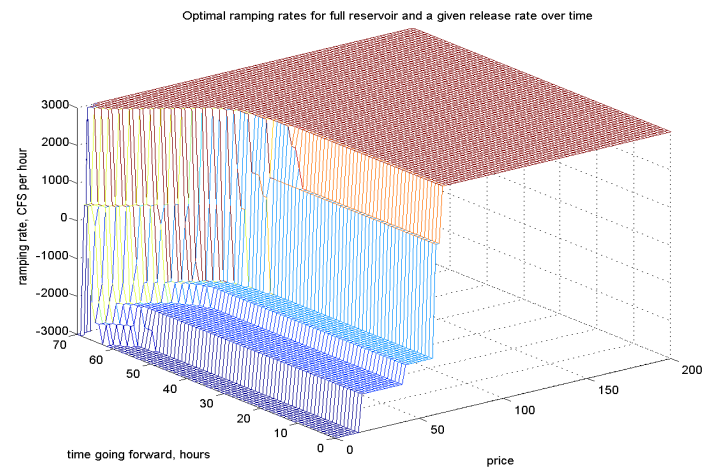

(a) Optimal Ramping Rate over Price and Time for the Base Regime

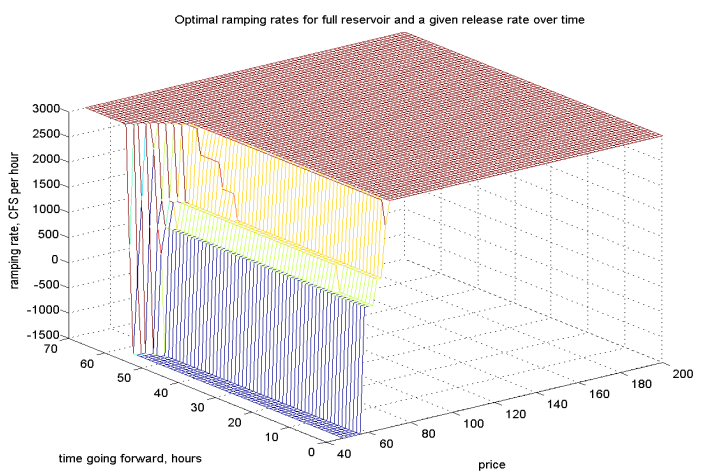

(b) Optimal Ramping Rate over Price and Time for the First Spike Regime

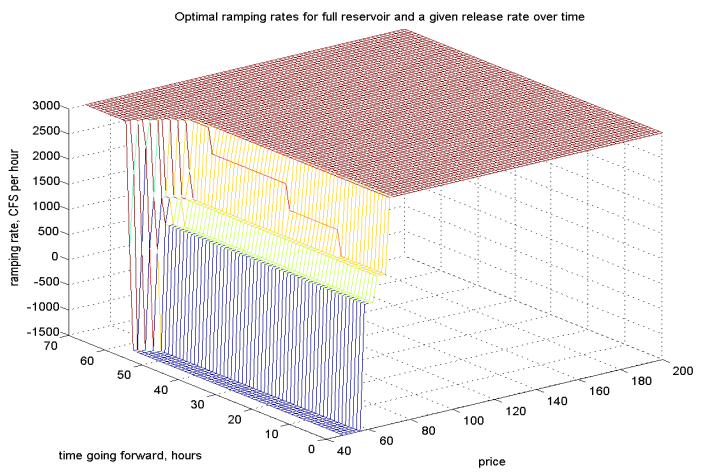

(c) Optimal Ramping Rate over Price and Time for the Second Spike Regime

Figure 4.9: Optimal Ramping Rate over Price and Time for the Base, the First Spike, and the Second Spike Regimes (Case II) 


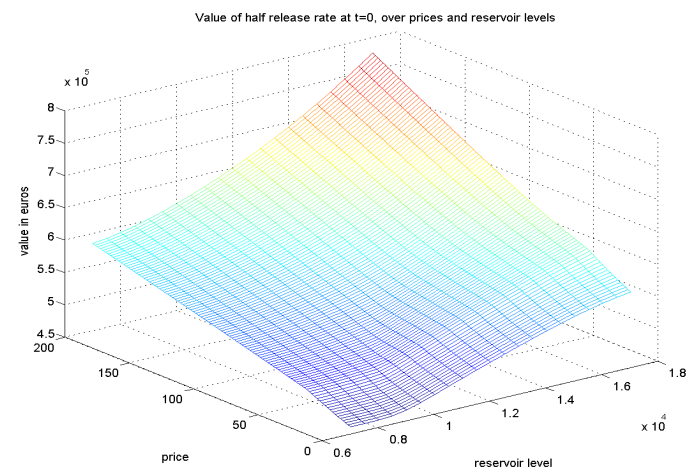

(a) Value over Price and Reservoir Level for the Base Regime

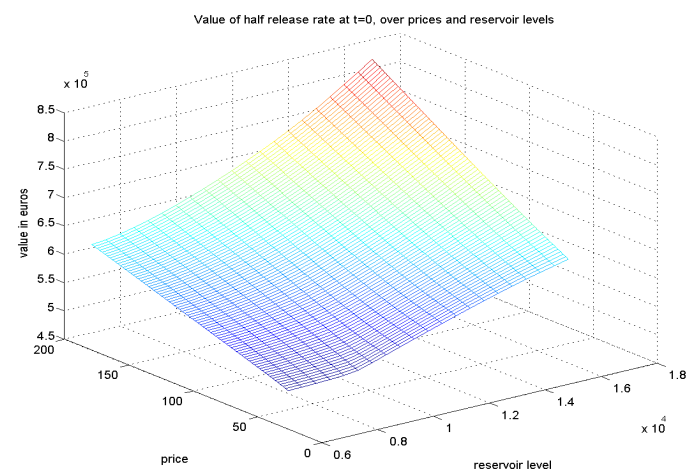

(b) Value over Price and Reservoir Level for the First Spike Regime

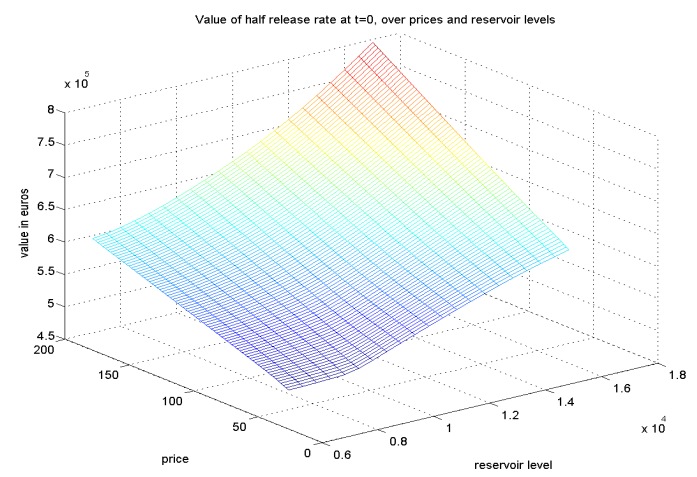

(c) Value over Price and Reservoir Level for the Second Spike Regime

Figure 4.10: Value over Price and Reservoir Level for the Base, the First Spike, and the Second Spike Regimes (Case II) 


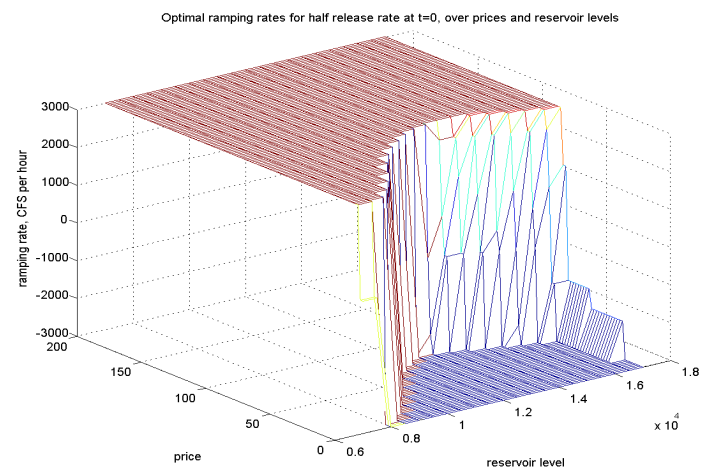

(a) Optimal Ramping Rate over Price and Reservoir Level for the Base Regime

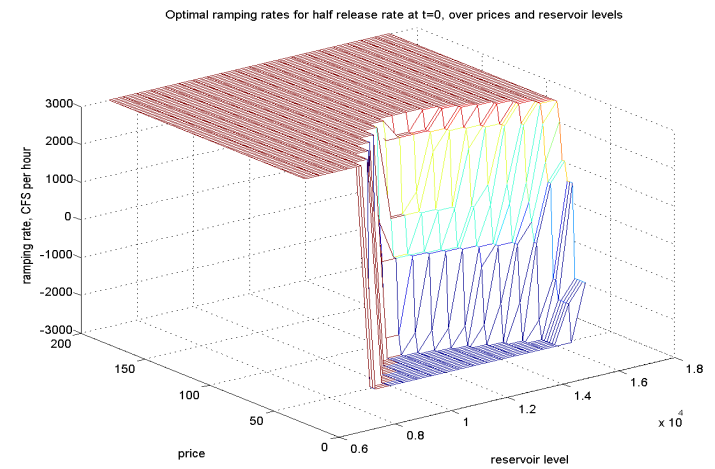

(b) Optimal Ramping Rate over Price and Reservoir Level for the First Spike Regime

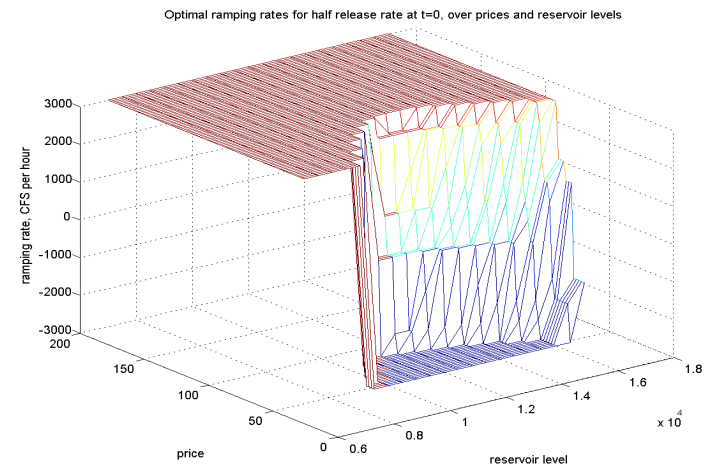

(c) Optimal Ramping Rate over Price and Reservoir Level for the Second Spike Regime

Figure 4.11: Optimal Ramping Rate over Price and Reservoir Level for the Base, the First Spike, and the Second Spike Regimes (Case 115 ) 
Table 4.3: Numerical Results Under Various Ramping Restrictions (Small Jump Size from the Base Regime to the Second Spike Regime)

\begin{tabular}{|c|c|c|c|c|c|c|}
\hline \multicolumn{2}{|l|}{ Case } & $\begin{array}{l}\text { No Ramping } \\
\text { Restrictions }\end{array}$ & 5000 (CFS-hr) & 3000 (CFS-hr) & 1000 (CFS-hr) & 250 (CFS-hr) \\
\hline \multirow[t]{2}{*}{$\mathrm{HF}$} & Profit & 616750 & 614740 & 611470 & 604670 & 582660 \\
\hline & $\%$ ch & $\mathrm{N} / \mathrm{A}$ & -0.3 & -0.9 & -2.0 & -5.5 \\
\hline \multirow[t]{2}{*}{ FF } & Profit & 615460 & 612230 & 606680 & 583220 & 569400 \\
\hline & $\%$ ch & $\mathrm{N} / \mathrm{A}$ & -0.5 & -1.4 & -5.2 & -7.5 \\
\hline \multicolumn{7}{|c|}{ Total Profit and Change of Total Profit in Regime 2 at Time 0 When the Initial Price is $80 \mathrm{EUR} / \mathrm{MWh}$} \\
\hline \multirow[t]{2}{*}{ HF } & Profit & 649420 & 646590 & 642330 & 632940 & 609570 \\
\hline & $\%$ ch & $\mathrm{N} / \mathrm{A}$ & -0.4 & -1.1 & -2.5 & -6.1 \\
\hline \multirow[t]{2}{*}{$\mathrm{FF}$} & Profit & 652120 & 649190 & 644430 & 624760 & 614090 \\
\hline & $\% \mathrm{ch}$ & $\mathrm{N} / \mathrm{A}$ & -0.5 & -1.2 & -4.2 & -5.8 \\
\hline \multicolumn{7}{|c|}{ "Total Profit and Change of Total Profit in Regime 2 at Time 0 When the Initial Price is 160 EUR/MWh } \\
\hline \multirow[t]{2}{*}{ HF } & Profit & 767400 & 762840 & 755890 & 735830 & 704220 \\
\hline & $\%$ ch & N/A & -0.6 & -1.5 & -4.1 & -8.2 \\
\hline \multirow[t]{2}{*}{ FF } & Profit & 780460 & 778230 & 774980 & 765980 & 764000 \\
\hline & $\% \mathrm{ch}$ & $\mathrm{N} / \mathrm{A}$ & -0.3 & -0.7 & -1.9 & -2.1 \\
\hline \multicolumn{7}{|c|}{ Total Profit and Change of Total Profit in Regime 3 at Time 0 When the Initial Price is $80 \mathrm{EUR} / \mathrm{MWh}$} \\
\hline \multirow[t]{2}{*}{$\mathrm{HF}$} & Profit & 643910 & 641070 & 636800 & 627600 & 604360 \\
\hline & $\%$ ch & N/A & -0.4 & -1.1 & -2.5 & -6.1 \\
\hline \multirow[t]{2}{*}{$\mathrm{FF}$} & Profit & 646630 & 643530 & 638520 & 617670 & 605860 \\
\hline & $\% \mathrm{ch}$ & $\mathrm{N} / \mathrm{A}$ & -0.5 & -1.3 & -4.5 & -6.3 \\
\hline \multicolumn{7}{|c|}{ Total Profit and Change of Total Profit in Regime 3 at Time 0 When the Initial Price is $160 \mathrm{EUR} / \mathrm{MWh}$} \\
\hline \multirow[t]{2}{*}{$\mathrm{HF}$} & Profit & 752470 & 748030 & 741280 & 722590 & 692680 \\
\hline & $\% \mathrm{ch}$ & $\mathrm{N} / \mathrm{A}$ & -0.6 & -1.5 & -4.0 & -8.0 \\
\hline \multirow[t]{2}{*}{ FF } & Profit & 765230 & 762940 & 759510 & 749410 & 746840 \\
\hline & $\% \mathrm{ch}$ & $\mathrm{N} / \mathrm{A}$ & -0.3 & -0.8 & -2.1 & -2.4 \\
\hline
\end{tabular}

Note: HF means half release rate and full reservoir level; FF means full release rate and full reservoir level. Profit is in Euros and \%ch refers to the percent change in profits compared to the case of no ramping restrictions. 


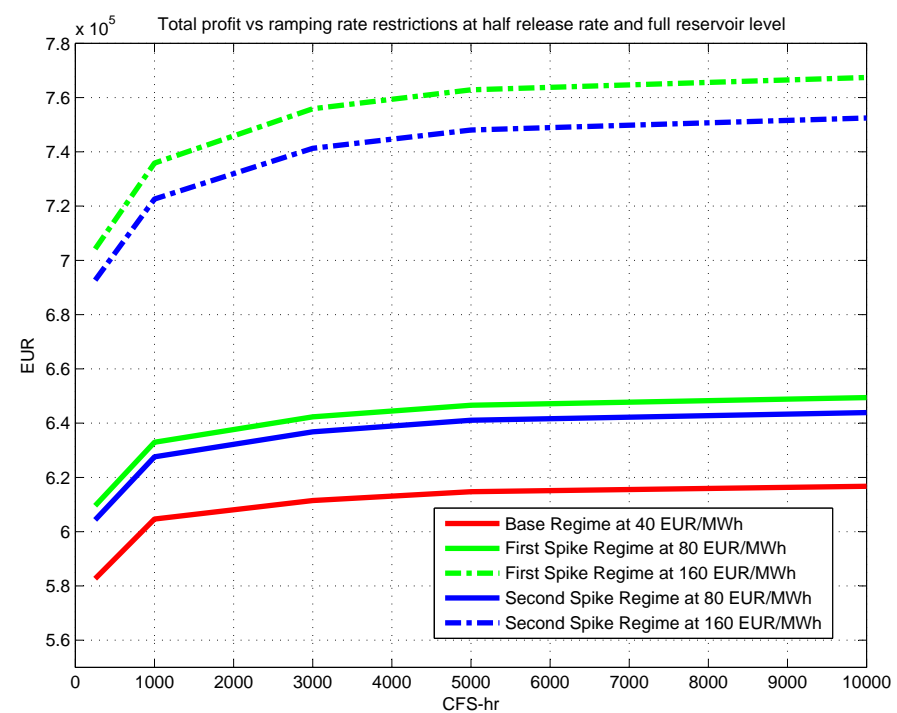

(a) Total Profit vs Ramping Rate Restrictions at Half Release Rate and Full Reservoir Level

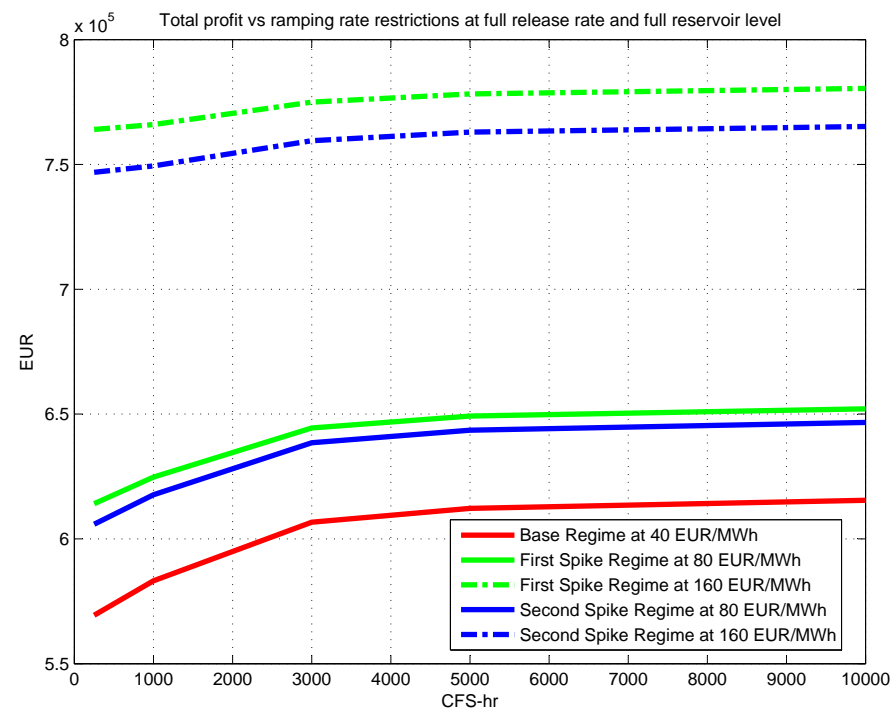

(b) Total Profit vs Ramping Rate Restrictions at Full Release Rate and Full Reservoir Level

Figure 4.12: Total Profit vs Ramping Rate Restrictions at Half Release Rate and Full Reservoir Level and at Full Release Rate and Full Reservoir Level (Case II) 


\subsection{Conclusions}

One of the major features of electricity price spikes is that when a jump occurs the price tends to jump up or down at a random size. To incorporate this characteristic into the regime switching model, we make a simple approximation of the random jump size by proposing a three regimes model (one base and two spike regimes) with multiple jump sizes. Within this framework, this chapter studies the effect of multiple jump sizes on the impact of ramping restrictions on a hydro power plant.

Using a prototype hydro dam, we valued the hydro plant for a range of ramping restrictions for the three regimes model for a three days period. Two numerical experiments are conducted for the multiple jump sizes: average jump size between the base regime and the first spike regime and larger than the average jump size between the base regime and the second spike regime; average jump size between the base regime and the first spike regime and smaller than the average jump size between the base regime and the second spike regime. As expected, these numerical experiments give consistent results as what we find in chapter 3. For both numerical experiments, we find that profits are significantly affected by less than $9 \%$ in the case of the most severe ramping constraints and for a range of less severe ramping constraints profits are impacted by less than $2 \%$. In addition, by introducing multiple jump sizes into the regime switching model, the results show that: (i) the present value in the spike regime with a large jump size is always higher than the present value in the spike regime with a small jump size; (ii) the small jump size case gives an overall lower value than the large jump size case; (iii) in the small jump size case ramping restrictions have a smaller impact than in the large jump size case.

This chapter adopted the Markov regime switching model and the associated parameter estimates found in the literature. We also made strong assumptions on other parameter values such as the transition intensities and the jump sizes. Further work is needed in estimating an electricity price model in the following directions: estimating the model in an hourly frequency; estimating the market price of risk; estimating the distribution of the jump size from one regime to another; improving the timing of spikes. 


\section{Chapter 5}

\section{Conclusions}

The ability of hydro facilities to respond quickly through ramping to changing demand and price conditions is one of the benefits of hydro power. However the significant negative consequences of ramping on aquatic ecosystems needs to be considered by regulators. These negative impacts are case specific, dependent on the physical structure of the dam and the ecological conditions of particular rivers and streams. In cases where ramping rate restrictions are being considered, apart from the environmental gains to the river ecosystem, there should be a recognition of the costs imposed on hydro operators in terms of lost profits as well as potential environmental impacts that result from the need to utilize alternative sources of electricity. Ideally ramping rate regulations would be determined through a careful analysis of all the potential impacts. This thesis contributes to our understanding of the impact of ramping restrictions on the hydro station's operation and profit and the trade offs involved.

In both non-stochastic and stochastic frameworks, the thesis finds highly consistent results on the impact of ramping restrictions on the hydro station's profit. For our mediumsized power plant profits are negatively affected by ramping restrictions. We observe profits are significantly affected (by less than $7 \%$ in chapter 2, by less than $10 \%$ in chapter 3 , and by less than $9 \%$ in chapter 4 ) in the case of the most severe ramping constraints, but we also observe a range of less severe ramping restrictions over which profits are not substantially 
affected (by less than $2 \%$ in chapter 2 , by less than $3 \%$ in chapter 3 , and by less than $2 \%$ in chapter 4).

In chapter two, we examine the effect of ramping restrictions imposed by a regulator on a hydro power plant's operation and profit. In a non-stochastic environment, we solve a dynamic non-linear model of the profit maximizing decision by a prototype hydro plant over 5 days given physical and environmental restrictions on water release rates. We assume the hydro plant must meet a minimum level of contract electricity demand through hydro production, purchasing on the spot market for resale, or some combinations of these two. Using data that is representative of a medium-sized hydro operation in Ontario, we investigate the operator's optimal decisions regarding hydro production and power purchases in on-peak and off-peak periods where on- and off-peak prices are assumed known and constant. In particular, we examine the sensitivity of hydro station profits to ramping restrictions as well as the potential impact on electricity production from hydro and other sources. The associated benefits and costs of ramping rate restrictions include the lost profit for the hydro operator, the environmental impact of changed reliance on thermal power, and the environmental benefit for the river ecosystem. Using the replacement power approach, we estimate the environmental cost (or benefit) of this change in thermal production due to the resulting change in air pollution emissions. In absence of monetary measure for the environmental benefit of ramping restrictions for the river ecosystem, we estimate the other two impacts to provide a lower bound for the environmental benefit of ramping rate restrictions that would be required in order for them to be worthwhile.

In this second chapter, our analysis shows ramping restrictions have a negative effect on profits since a hydro operator is forced to make different choices (on hydro production and power purchases) than when no ramping restrictions are imposed. In particular profits are reduced if ramping restrictions force hydro operators to increase hydro production in off-peak periods when prices are lower. A more surprising result is that ramping restrictions can cause an increase in the total amount of hydro power produced for a given day. This result follows from the desire of the hydro operator to mitigate the effect of the ramping constraints by increasing power production in off-peak periods while at the same time attempting to maintain production as much as possible in on-peak periods. Under 
the replacement power assumption, the increased hydro production results in a reduction in air pollution emissions. This constitutes an added environmental benefit of ramping restrictions, in addition to any benefits to the river ecosystem. We calculate a net cost of the ramping restrictions as the lost profits net of any environmental benefit of reduced air pollution. This net cost can be compared to expected environmental benefits from an improved aquatic ecosystem.

In chapter three, we investigate the impact of ramping restrictions on hydro plant operations and profitability using a Markov regime switching model of electricity prices. Hydro operations are formulated as a stochastic control problem subject to various physical and environmental constraints which results in the HJB-PDE for the value of the hydro station. The optimal control is obtained by solving the HJB-PDE numerically using a fully implicit finite difference approach with semi-Lagrangian time stepping. For the same prototype hydro dam as chapter 2 we value the power plant and model the lost profits for a range of ramping restrictions for the regime switching model over a one week period. We pay special attention to how the impact of ramping restrictions depends on the marginal cost of hydroelectric power and the characteristics of the assumed price process, and how the presence of two price regimes affects hydro plant value and optimal operations. In the stochastic environment, it is notoriously difficult to measure the environmental cost (or benefit) of alternate thermal power generation when ramping restrictions are imposed. In absence of monetary measure for the environmental benefit of ramping restrictions for the aquatic ecosystem, we seek to examine the other side of the equation - the costs of these restrictions in terms of lost profitability. Knowledge of these costs will help illuminate the trade offs involved and inform the design of regulations.

In this third chapter, we find that in most scenarios the hydro plant will ramp up or down at the maximum allowed rates. The exception to this is when the dam is up against one of the other constraints such as maximum/minimum release rates or maximum water content in the dam. The sensitivity analysis shows that ramping restrictions have a larger effect in an environment where frequent ramping up or down is desired. A lower speed of mean reversion, higher volatility and more frequent transition to the spike regime will increase the impact of ramping restrictions. One conclusion is that the ramping effect on 
profits mostly depends on the price relative to the cost of generation (how long and how frequent the price is close to or below the cost), but is not very sensitive to the price jumps.

In chapter four, we study the impact of ramping restrictions on hydro plant operations and profitability by extending the regime switching model in chapter 3 to a model with multiple jump sizes. Specifically, we propose a regime switching model with three regimes among which the jump sizes are different. For the same prototype hydro dam as chapter 2 we examine the effect of multiple jump sizes on the impact of ramping restrictions on the hydro plant's operation and profit over 3 days. In this fourth chapter, the two numerical experiments provide further evidence that ramping restrictions have a larger impact when the expected variation in price is increased such as through an increase in jump size (for the third regime) which makes it desirable to change water release rates relatively frequently.

All of these results in this thesis will depend on the specifics of the particular hydro plant under consideration such as the physical structure as well as the market structure that the plant operates in such as the pricing system. In general, these empirical findings are quite robust under different specifications for electricity prices. An important conclusion of the thesis is that ramping restrictions should not be determined in isolation, but rather using a cost-benefit approach that evaluates the trade offs involved. This thesis has identified some of the important trade offs that should be examined more carefully in future research. These include the impact on hydro plant's operation and profit as well as the environmental impact of a change in the intensity of use of other types of power.

In practice, to determine the appropriate ramping restrictions for a specific hydro plant a regulator should consider the following steps. We should start by specifying the functional form for the generation of hydroelectric power and then estimate the parameter values for this function using historical data including the power output, water flow rate, water head, water content and generation efficiency. Next, we can assume and estimate the stochastic processes for electricity prices based on hourly data from the spot market where the hydro plant sells its power. Now the associated benefits and costs of ramping restrictions could be calculated by using the methods proposed in this thesis. These include the lost profit for the hydro plant and the environmental externality of the replacement thermal power (using the marginal external cost of emissions for that market). It is noticed that the cost- 
benefit analysis is not complete unless we could put a dollar value on the environmental benefit of ramping restrictions on the river ecosystem. However, currently there are no studies available to directly provide monetary measures of the environmental benefit of ramping restrictions ${ }^{1}$. To address this issue, one possible option is to use the contingent valuation (CV) approach to obtain the willingness to pay (WTP) values of restricting the ramping rate of the hydro operator to protect the river ecosystem. If this experiment at the policy site is not feasible due to the time and resource constraints, then the benefit transfer approach could be used to calculate these values when similar studies at another place and time are available. However, adjustments are normally required for the consistency between the study and policy sites with respect to commodities, markets, and welfare measures. In addition, to design the ramping policy the regulator should also consider the environmental scientists' views which are based on the scientific evidence from biological studies.

There are several directions for further research. First, we could account for uncertainty in water inflow and solve the HJB-PDE with two dimensional stochastic processes for the electricity price and water inflow. Second, further work is needed in estimating an electricity price model in the following directions: estimating the model in an hourly frequency; estimating the market price of risk; estimating the distribution of the up- and down-jump sizes; improving the timing of spikes. Third, further efforts are needed to construct a monetary measure of the environmental benefits for the river ecosystem when ramping restrictions are imposed. Fourth, an alternative hydro dam with different physical structure and power production function could be used. Finally, the ramping issue could be better studied through a partial equilibrium model of hydro-thermal competition. This would allow us to analyze the impact of ramping restrictions on the equilibrium price and production, associated pollutant emission of thermal power and social welfare.

\footnotetext{
${ }^{1}$ In this case, it becomes very important to obtain an accurate measure of the associated costs of ramping restrictions such as the lost profit for the hydro plant. Regulators and industry can use this information to negotiate the possible compensation for the hydro plant and the optimal ramping rate for environmental and economic benefits.
} 
APPENDICES 


\section{Appendix A}

\section{Appendix to Chapter 3}

\section{A.1 Derivation and Numerical Algorithms of HJB- PDE}

\section{A.1.1 Derivation of HJB-PDE}

Given Equation (3.21), hedging arguments are used to derive the HJB-PDE, Equation (3.23), describing the value of the hydro dam. The derivation of Equation (3.23) is based on Kennedy [2007] who analyzes the pricing of financial derivatives under regime switching prices. This derivation is also based on Chen and Forsyth [2008] who consider optimal control of a hydro dam assuming prices follow jump diffusion.

A target option $V$ is hedged with a portfolio of $N$ instruments $\left\{\Gamma_{\bar{n}}\right\}_{\bar{n}=1}^{N}$ whose values depend on the underlying electricity price $P$. There is a bank account $B$ that earns the risk free rate $\bar{r}$. The value of the hedge position is

$$
\Pi=-V+\sum_{\bar{n}=1}^{N} \varpi_{\bar{n}} \Gamma_{\bar{n}}+B .
$$

where $\varpi_{\bar{n}}$ is the weight for the $\bar{n}$ th hedging instrument. If the Markov chain is now in state 
$\imath$, the instantaneous change in the target option over an instant $d t$ is obtained using Itô's lemma:

$$
\begin{aligned}
V^{\imath}(P+d P, w+d w, r+d r, t+d t)= & V^{\imath}+V_{t}^{\imath} d t+V_{r}^{\imath} d r+V_{w}^{\imath} d w+V_{P}^{\imath} d P+\frac{1}{2} V_{r r}^{\imath} d r d r \\
& +\frac{1}{2} V_{w w}^{\imath} d w d w+\frac{1}{2} V_{P P}^{\imath} d P d P+V_{P w}^{\imath} d P d w \\
& +V_{P r}^{\imath} d P d r+V_{w r}^{\imath} d w d r .
\end{aligned}
$$

In addition, the profit generated over the instant $d t$ is given by

$$
H(r, w) q(r, h(w))(P-c) d t
$$

Making the appropriate substitutions in Equation (A.2) for $d w, d r$ and $d P$, we have

$$
d V=\hat{\mu}^{\imath} d t+\hat{\sigma}^{\imath} d Z+\sum_{\substack{\jmath=1 \\ \jmath \neq \imath}}^{N} \Delta V^{\imath \jmath} d X_{\imath \jmath}
$$

where

$$
\begin{aligned}
& \hat{\mu}^{\imath}=\left[\frac{\partial V^{\imath}}{\partial t}+z \frac{\partial V^{\imath}}{\partial r}+H(r, w) a(\ell-r) \frac{\partial V^{\imath}}{\partial w}+\mu^{\imath}(P, t) \frac{\partial V^{\imath}}{\partial P}+\right. \\
& \left.\frac{1}{2}\left(\sigma^{\imath}\right)^{2}(P, t) \frac{\partial^{2} V^{\imath}}{\partial P^{2}}+H(r, w) q(r, h(w))(P-c)\right] . \\
& \hat{\sigma}^{\imath}=\sigma^{\imath}(P, t) \frac{\partial V^{\imath}}{\partial P} . \\
& \Delta V^{\imath \jmath}=V^{\jmath}-V^{\imath} . \\
& V^{\kappa}=V^{\kappa}\left(P \xi^{\imath \kappa}, w, r, t\right) .
\end{aligned}
$$

Similarly for each of the hedging instruments, we have

$$
d \Gamma_{\bar{n}}=\bar{\mu}_{\bar{n}}^{\imath} d t+\bar{\sigma}_{\bar{n}}^{\imath} d Z+\sum_{\substack{\jmath=1 \\ \jmath \neq \imath}}^{N} \Delta \Gamma_{\bar{n}}^{\imath \jmath} d X_{\imath \jmath}
$$


where

$$
\begin{aligned}
& \bar{\mu}_{\bar{n}}^{\imath}=\left[\frac{\partial \Gamma_{\bar{n}}^{\imath}}{\partial t}+\mu^{\imath}(P, t) \frac{\partial \Gamma_{\bar{n}}^{\imath}}{\partial P}+\frac{1}{2}\left(\sigma^{\imath}\right)^{2}(P, t) \frac{\partial^{2} \Gamma_{\bar{n}}^{\imath}}{\partial P^{2}}\right] . \\
& \bar{\sigma}_{\bar{n}}^{\imath}=\sigma^{\imath}(P, t) \frac{\partial \Gamma_{\bar{n}}^{\imath}}{\partial P} . \\
& \Delta \Gamma_{\bar{n}}^{\imath \jmath}=\Gamma_{\bar{n}}^{\jmath}-\Gamma_{\bar{n}}^{\imath} . \\
& \Gamma_{\bar{n}}^{\kappa}=\Gamma_{\bar{n}}^{\kappa}\left(P \xi^{\kappa}, t\right) .
\end{aligned}
$$

Now we use equations for $d V$ and $d \Gamma_{\bar{n}}$ to specify the instantaneous change in the value of the hedging portfolio which is

$$
\begin{aligned}
d \Pi= & -d V+\sum_{\bar{n}=1}^{N} \varpi_{\bar{n}} d \Gamma_{\bar{n}}+d B \\
= & {\left[-\hat{\mu}^{\imath}+\sum_{\bar{n}=1}^{N} \varpi_{\bar{n}} \bar{\mu}_{\bar{n}}^{\imath}+\bar{r} B\right] d t+\left[-\hat{\sigma}^{\imath}+\sum_{\bar{n}=1}^{N} \varpi_{\bar{n}} \bar{\sigma}_{\bar{n}}^{\imath}\right] d Z } \\
& +\sum_{\substack{\jmath=1 \\
\jmath \neq \imath}}^{N}\left[-\Delta V^{\imath \jmath}+\sum_{\bar{n}=1}^{N} \varpi_{\bar{n}} \Delta \Gamma_{\bar{n}}^{\imath \jmath}\right] d X_{\imath \jmath} .
\end{aligned}
$$

In order to hedge both the diffusion risk (one equation) and the regime switching risk ( $N-1$ equations), the following $N$ linear equations need to hold

$$
\begin{gathered}
\sum_{\bar{n}=1}^{N} \varpi_{\bar{n}} \bar{\sigma}_{\bar{n}}^{\imath}=\hat{\sigma}^{\imath} . \\
\sum_{\bar{n}=1}^{N} \varpi_{\bar{n}} \Delta \Gamma_{\bar{n}}^{\imath \jmath}=\Delta V^{\imath \jmath} \jmath=1,2, \ldots, N ; \jmath \neq \imath .
\end{gathered}
$$

After eliminating both the diffusion risk and the regime switching risk, the hedging portfolio should earn the risk free rate of return to avoid arbitrage opportunities. This implies that 
$d \Pi=\bar{r} \Pi d t$. Using Equation (A.1) for the portfolio value $\Pi$ and Equation (A.6) for its differential form $d \Pi$, the non-arbitrage condition is given by

$$
\sum_{\bar{n}=1}^{N} \varpi_{\bar{n}}\left(\bar{\mu}_{\bar{n}}^{\imath}-\bar{r} \Gamma_{\bar{n}}^{\imath}\right)=\hat{\mu}^{\imath}-\bar{r} V^{\imath} .
$$

Now, we have a linear system consisting $N+1$ equations and $N$ unknowns.

$$
\left[\begin{array}{cccc}
\bar{\sigma}_{1}^{\imath} & \bar{\sigma}_{2}^{\imath} & \cdots & \bar{\sigma}_{N}^{\imath} \\
\Delta \Gamma_{1}^{\imath 1} & \Delta \Gamma_{2}^{\imath 1} & \cdots & \Delta \Gamma_{N}^{\imath 1} \\
\Delta \Gamma_{1}^{\imath 2} & \Delta \Gamma_{2}^{\imath 2} & \cdots & \Delta \Gamma_{N}^{\imath 2} \\
\vdots & \vdots & \ddots & \vdots \\
\Delta \Gamma_{1}^{\imath(\imath-1)} & \Delta \Gamma_{2}^{\imath(\imath-1)} & \cdots & \Delta \Gamma_{N}^{\imath(\imath-1)} \\
\Delta \Gamma_{1}^{\imath(\imath+1)} & \Delta \Gamma_{2}^{\imath(\imath+1)} & \cdots & \Delta \Gamma_{N}^{\imath(\imath+1)} \\
\vdots & \vdots & \ddots & \vdots \\
\Delta \Gamma_{1}^{\imath N} & \Delta \Gamma_{2}^{\imath N} & \cdots & \Delta \Gamma_{N}^{\imath N} \\
\bar{\mu}_{1}^{\imath}-\bar{r} \Gamma_{1}^{\imath} & \bar{\mu}_{2}^{\imath}-\bar{r} \Gamma_{2}^{\imath} & \cdots & \bar{\mu}_{N}^{\imath}-\bar{r} \Gamma_{N}^{\imath}
\end{array}\right]\left[\begin{array}{c}
\varpi_{1} \\
\varpi_{2} \\
\varpi_{3} \\
\vdots \\
\vdots \\
\vdots \\
\vdots \\
\varpi_{N-1} \\
\varpi_{N}
\end{array}\right]=\left[\begin{array}{c}
\hat{\sigma}^{\imath} \\
\Delta V^{\imath 1} \\
\Delta V^{\imath 2} \\
\vdots \\
\Delta V^{\imath(\imath-1)} \\
\Delta V^{\imath(\imath+1)} \\
\vdots \\
\Delta V^{\imath N} \\
\hat{\mu}^{\imath}-\bar{r} V^{\imath}
\end{array}\right]
$$

Hence, this matrix system is over specified. To obtain a solution this system must be linearly dependent. We assume the following linear combination of the system of equations

$$
\Lambda^{\imath} \times R_{1}-\lambda_{\imath 1}^{Q} \times R_{2}-\lambda_{\imath 2}^{Q} \times R_{3}-\ldots-\lambda_{\imath N}^{Q} \times R_{N}-R_{N+1} .
$$

where $R_{\text {J }}$ refers to the th row of the matrix system above. This means that each element in (A.7) must be zero for some choice of $\Lambda^{\imath}$ and $\left\{\lambda_{\imath \jmath}^{Q}\right\}_{\jmath=1, \jmath \neq \imath}^{N}$. Therefore, following Kennedy [2007] (Appendix A), we obtain another matrix system given by 


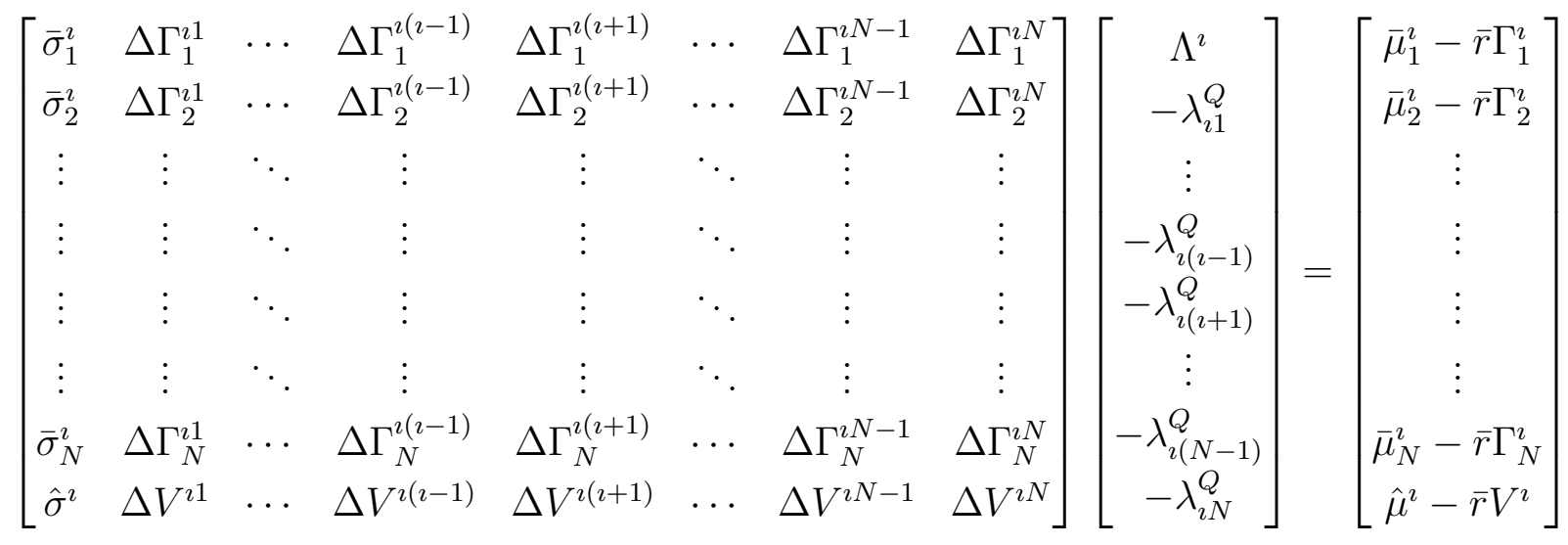

In this matrix system, the linear equations are independent to each other. We take the last equation from this matrix and get

$$
\Lambda^{\imath} \hat{\sigma}^{\imath}-\sum_{\substack{\jmath=1 \\ \jmath \neq \imath}}^{N} \lambda_{\imath \jmath}^{Q} \Delta V^{\imath \jmath}=\hat{\mu}^{\imath}-\bar{r} V^{\imath}
$$

Substituting $\hat{\mu}^{\imath}, \hat{\sigma}^{\imath}$ and $\Delta V^{\imath \jmath}$ from Equation (A.3) into Equation (A.8), we get

$$
\begin{array}{r}
0=\frac{\partial V^{\imath}}{\partial t}+\sup _{z \in Z(r)}\left(z \frac{\partial V^{\imath}}{\partial r}\right)+H(r, w) a(\ell-r) \frac{\partial V^{\imath}}{\partial w}+\frac{1}{2}\left(\sigma^{\imath}\right)^{2}(P, t) \frac{\partial^{2} V^{\imath}}{\partial P^{2}}+\left(\mu^{\imath}(P, t)-\Lambda^{\imath} \sigma^{\imath}(P, t)\right) \frac{\partial V^{\imath}}{\partial P} \\
+H(r, w) q(r, h(w))(P-c)-\bar{r} V^{\imath}+\sum_{\substack{\jmath=1 \\
\jmath \neq \imath}}^{N} \lambda_{\imath \jmath}^{Q}\left(V^{\jmath}-V^{\imath}\right) .
\end{array}
$$

Following the same procedure, the system of PDE's for $\imath=1,2, \ldots, N$ could be derived. Recall that $\Lambda^{2}$ is the market price of risk and is multiplied by the volatility term, $\sigma^{2}(P, t)$, and deducted from the drift, $\mu^{\imath}(P, t)$, giving the risk adjusted drift for price in regime $\imath$. The $\lambda_{\imath \jmath}^{Q}$ 's $(\jmath \neq \imath)$ are the risk adjusted transition intensity of a regime switch. These may be contrasted with the real world transition intensities, $q_{\imath \jmath}$ specified in Equation (3.2). 


\section{A.1.2 Numerical Algorithms}

We use equally spaced grids in $P, w$ and $r$ directions for the PDE discretization, respectively denoted by $\left[P_{0}, P_{1}, \ldots, P_{i_{\max }}\right],\left[w_{0}, w_{1}, \ldots, w_{j_{\max }}\right]$ and $\left[r_{0}, r_{1}, \ldots, r_{k_{\max }}\right]$ with $P_{0}=0, P_{i_{\max }}=$ $P^{\max }, w_{0}=w^{\min }, w_{j_{\max }}=w^{\max }, r_{0}=r^{\min }$ and $r_{k_{\max }}=r^{\max }$. When the jump size is greater than 1 , the price may move above $P^{\max }$. To avoid the price jumping out of the computational domain, we use an augmented jump amplitude near $P^{\max }$ (as done by Kennedy [2007]) and define $\bar{\xi}^{\imath \jmath}(P)$ as follows

$$
\bar{\xi}^{\imath \jmath}(P)= \begin{cases}\xi^{\imath \jmath} & \text { if } 0 \leq P \leq \frac{P^{\max }}{\xi^{\imath \jmath}} \\ \frac{P^{\max }}{P} & \text { if } \frac{P^{\max }}{\xi^{\imath \jmath}}<P \leq P^{\max }\end{cases}
$$

Now $P \bar{\xi}^{\imath \jmath}(P)$ will remain in $\left[0, P^{\max }\right]$.

Let $0=0 \Delta \tau<\ldots<\aleph \Delta \tau=T$ be the discrete timesteps and $\tau^{n}=n \Delta \tau$ denote the $n$th timestep. Let $V^{\imath}\left(P_{i}, w_{j}, r_{k}, \tau^{n}\right)$ denote the exact solution of the pricing equation when the electricity spot price is $P_{i}$, the water content is $w_{j}$, the outflow rate is $r_{k}$, the discrete time is $\tau^{n}$, and the regime is $\imath$. Let $V_{i, j, k}^{\imath, n}$ denote an approximation of the exact solution $V^{\imath}\left(P_{i}, w_{j}, r_{k}, \tau^{n}\right)$.

We use standard finite difference methods to discretize the operator $C_{0} V^{\imath}, C V^{\imath}$ and $C_{1} V^{\imath}$ as defined in Sections 3.5.1 and 3.5.2. Following Chen and Forsyth [2007, 2008], let $\left(C_{\varepsilon} V\right)_{i, j, k}^{\imath, n}$ denote the discrete value of the differential operators $C_{0} V^{\imath}, C V^{\imath}$ or $C_{1} V^{\imath}$ at a node $\left(P_{i}, w_{j}, r_{k}, \tau^{n}\right)$ in regime $\imath$, so that $\left(C_{\varepsilon} V\right)_{i, j, k}^{\imath, n}$ is an approximation for $\left(C_{0} V\right)_{i, j, k}^{\imath, n}$ if $P_{i}=0$, an approximation for $(C V)_{i, j, k}^{\imath, n}$ if $P_{i} \in\left(0, P^{\max }\right)$, and an approximation for $\left(C_{1} V\right)_{i, j, k}^{\imath, n}$ if $P_{i}=$ $P^{\max }$. The operators can be discretized using central, forward, or backward differencing in the $P$ direction to give 


$$
\begin{aligned}
\left(C_{\varepsilon} V\right)_{i, j, k}^{\imath, n} & =\gamma_{i}^{\imath} V_{i-1, j, k}^{\imath, n}+\beta_{i}^{\imath} V_{i+1, j, k}^{\imath, n}-\left(\gamma_{i}^{\imath}+\beta_{i}^{\imath}+\left(\bar{r}+\sum_{\substack{j=1 \\
\jmath \neq \imath}}^{N} \lambda_{\imath \jmath}^{Q}\right)\right) V_{i, j, k}^{\imath, n} \text { if } P_{i} \in\left(0, P^{\max }\right) \\
& =\beta_{i}^{\imath} V_{i+1, j, k}^{\imath, n}-\left(\beta_{i}^{\imath}+\left(\bar{r}+\sum_{\substack{\jmath=1 \\
\jmath \neq \imath}}^{N} \lambda_{\imath \jmath}^{Q}\right)\right) V_{i, j, k}^{\imath, n} \text { if } P_{i}=0 \\
& =\gamma_{i}^{\imath} V_{i-1, j, k}^{\imath, n}-\left(\gamma_{i}^{\imath}+\left(\bar{r}+\sum_{\substack{\jmath=1 \\
\jmath \neq \imath}}^{N} \lambda_{\imath \jmath}^{Q}\right)\right) V_{i, j, k}^{\imath, n} \text { if } P_{i}=P^{\max }
\end{aligned}
$$

where $\gamma_{i}^{\imath}$ and $\beta_{i}^{\imath}$ are defined in Appendices A.1.7, A.1.8, and A.1.9. The algorithm uses central differencing as much as possible at each node since it is more accurate (d'Halluin et al. [2004]). The use of forward or backward differencing guarantees $\gamma_{i}^{\imath}$ and $\beta_{i}^{\imath}$ satisfy the following positive coefficient conditions: $\gamma_{i}^{\imath} \geq 0$ and $\beta_{i}^{\imath} \geq 0$ for $i=0, \cdots, i_{\max }, \imath=1, \cdots, N$.

Let $\left(B_{\varepsilon} V\right)_{i, j, k}^{\imath, n}$ be an approximation of the operator $B V^{\imath}$ at a mesh node $\left(P_{i}, w_{j}, r_{k}, \tau^{n}\right)$. For $B V^{\imath}=\sum_{\substack{\jmath=1 \\ \jmath \neq \imath}}^{N} \lambda_{\imath \jmath}^{Q} V^{\jmath}$, we have

$$
\left(B_{\varepsilon} V\right)_{i, j, k}^{\imath, n}=\sum_{\substack{\jmath=1 \\ \jmath \neq \imath}}^{N} \lambda_{\imath \jmath}^{Q} \chi\left(P_{i} \bar{\xi}^{\imath \jmath}\left(P_{i}\right), V_{i_{\jmath}^{*}, j, k}^{\jmath, n}, V_{i_{\jmath}^{*}+1, j, k}^{\jmath, n}\right) .
$$

where, $V^{\jmath}=V^{\jmath}\left(P_{i} \bar{\xi}^{\imath \jmath}\left(P_{i}\right), w_{j}, r_{k}, \tau^{n}\right)$ is approximated by the linear interpolation using $V_{i_{\jmath}^{*}, j, k}^{\jmath, n}$ and $V_{i_{\jmath}^{*}+1, j, k}^{\jmath, n}$, written as $\chi\left(P_{i} \bar{\xi}^{\imath \jmath}\left(P_{i}\right), V_{i_{\jmath}^{*}, j, k}^{\jmath, n}, V_{i_{\jmath}^{*}+1, j, k}^{\jmath, n}\right)$ with $P_{i_{\jmath}^{*}} \leq P_{i} \bar{\xi}^{\natural \jmath}\left(P_{i}\right) \leq P_{i_{\jmath}^{*}+1}$.

\section{A.1.3 Numerical Scheme for Pricing Equations}

Following Chen and Forsyth [2007, 2008], we discretize the terms in PDE (3.23) using a semi-Lagrangian time-stepping. Regarding $P$ as fixed, then in regime $\imath$ the total (La- 
grangian) derivative for $V^{\imath}=V^{\imath}(P, w(\tau), r(\tau), \tau)$ along a trajectory $w=w(\tau), r=r(\tau)$ is

$$
\begin{aligned}
\frac{D V^{\imath}}{D \tau} & =\frac{\partial V^{\imath}}{\partial \tau}+\frac{\partial V^{\imath}}{\partial r} \frac{d r}{d \tau}+\frac{\partial V^{\imath}}{\partial w} \frac{d w}{d \tau} \\
& =\frac{\partial V^{\imath}}{\partial \tau}-\frac{\partial V^{\imath}}{\partial r} \frac{d r}{d t}-\frac{\partial V^{\imath}}{\partial w} \frac{d w}{d t} \\
& =\frac{\partial V^{\imath}}{\partial \tau}-z \frac{\partial V^{\imath}}{\partial r}-H(r, w) a(\ell-r) \frac{\partial V^{\imath}}{\partial w}
\end{aligned}
$$

Here we use the dynamics of $w$ in Equation (3.6) and $r$ in Equation (3.9). Now Equation (3.23) can be written as

$$
\frac{D V^{\imath}}{D \tau}=C V^{\imath}+B V^{\imath}+H(r, w) q(r, h(w))(P-c) .
$$

Let $\zeta_{i, j, k}^{\imath, n+1}$ denote the value of the control variable $z$ at the mesh node $\left(P_{i}, w_{j}, r_{k}, \tau^{n+1}\right)$. At $\left(P_{i}, w_{j}, r_{k}, \tau^{n+1}\right), \frac{D V^{\imath}}{D \tau}$ is approximated by

$$
\left(\frac{D V}{D \tau}\right)_{i, j, k}^{\imath, n+1}=\frac{1}{\Delta \tau}\left(V_{i, j, k}^{\imath, n+1}-V_{i, \hat{j}, \hat{k}}^{\imath, n}\right)+\text { truncation error } .
$$

where $V_{i, \hat{j}, \hat{k}}^{\imath, n}$ is an approximation of $V^{\imath}\left(P_{i}, w_{\hat{j}}^{n}, r_{\hat{k}}^{n}, \tau^{n}\right)$ obtained by linear interpolation with $w_{\hat{j}}^{n}$ and $r_{\hat{k}}^{n}$ given by

$$
\begin{gathered}
w_{\hat{j}}^{n}=\min \left[\max \left[w_{j}+H\left(r_{k}, w_{j}\right) a\left(\ell-r_{k}\right) \Delta \tau, w^{\min }\right], w^{\max }\right] . \\
r_{\hat{k}}^{n}=r_{k}+\zeta_{i, j, k}^{\imath, n+1} \Delta \tau .
\end{gathered}
$$

Equation (A.15) guarantees that $w_{\hat{j}}^{n}$ will remain in the domain $\left[w^{\min }, w^{\max }\right]$. The control $\zeta_{i, j, k}^{\imath, n+1}$ must satisfy the constraint $\zeta_{i, j, k}^{i, n+1} \in Z\left(r_{k}\right)$. Let $Z_{k} \subseteq Z\left(r_{k}\right)$ denote the set of values of $\zeta_{i, j, k}^{\imath, n+1} \in Z\left(r_{k}\right)$ such that the resulting $r_{\hat{k}}^{n}$ computed from (A.16) is bounded within $\left[r^{\min }, r^{\max }\right]$. 
Now at any discrete mesh node $\left(P_{i}, w_{j}, r_{k}, \tau^{n+1}\right), n \geq 0$, PDE (3.23) and the associated boundary Equations (3.25) and (3.26) can be discretized as

$V_{i, j, k}^{\imath, n+1}=\sup _{\zeta_{i, j, k}^{\imath, n+1} \in Z_{k}} V_{i, \hat{j}, \hat{k}}^{\imath, n}+\Delta \tau\left(C_{\varepsilon} V\right)_{i, j, k}^{\imath, n+1}+\Delta \tau\left(B_{\varepsilon} V\right)_{i, j, k}^{\imath, n+1}+\Delta \tau H\left(r_{k}, w_{j}\right) q\left(r_{k}, h\left(w_{j}\right)\right)\left(P_{i}-c\right)$.

Substituting $\left(C_{\varepsilon} V\right)_{i, j, k}^{\imath, n+1}$ from (A.10) and $\left(B_{\varepsilon} V\right)_{i, j, k}^{\imath, n+1}$ from (A.11) into (A.17), we get

$$
\begin{aligned}
& -\Delta \tau \gamma_{i}^{\imath} V_{i-1, j, k}^{\imath, n+1}+\left[1+\Delta \tau\left(\gamma_{i}^{\imath}+\beta_{i}^{\imath}+\left(\bar{r}+\sum_{\substack{j=1 \\
j \neq \imath}}^{N} \lambda_{\imath \jmath}^{Q}\right)\right)\right] V_{i, j, k}^{\imath, n+1}-\Delta \tau \beta_{i}^{\imath} V_{i+1, j, k}^{\imath, n+1}
\end{aligned}
$$

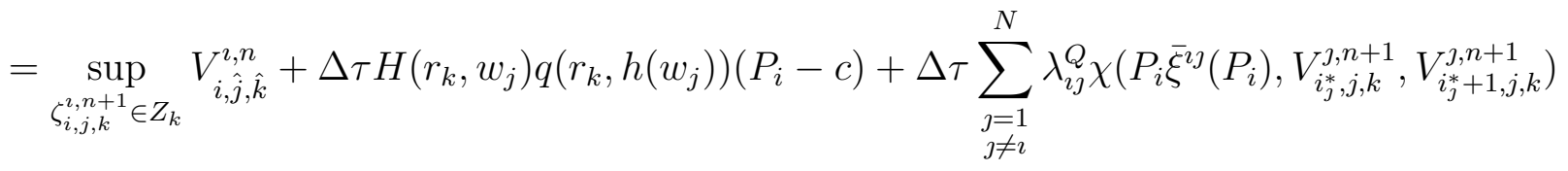

$$
\begin{aligned}
& \text { if } P_{i} \in\left(0, P^{\max }\right) \\
& {\left[1+\Delta \tau\left(\beta_{i}^{\imath}+\left(\bar{r}+\sum_{\substack{j=1 \\
j \neq \imath}}^{N} \lambda_{\imath \jmath}^{Q}\right)\right)\right] V_{i, j, k}^{\imath, n+1}-\Delta \tau \beta_{i}^{\imath} V_{i+1, j, k}^{\imath, n+1}} \\
& =\sup _{\zeta_{i, j, k}^{,, n+1} \in Z_{k}} V_{i, \hat{j}, \hat{k}}^{\imath, n}-\Delta \tau H\left(r_{k}, w_{j}\right) q\left(r_{k}, h\left(w_{j}\right)\right) c+\Delta \tau \sum_{\substack{\jmath=1 \\
\jmath \neq \imath}}^{N} \lambda_{\imath \jmath}^{Q} \chi\left(P_{i} \bar{\xi}^{\imath \jmath}\left(P_{i}\right), V_{i_{\jmath}^{*}, j, k}^{\jmath, n+1}, V_{i_{j}^{*}+1, j, k}^{\jmath, n+1}\right) \\
& \text { if } P_{i}=0
\end{aligned}
$$




$$
\begin{array}{r}
-\Delta \tau \gamma_{i}^{\imath} V_{i-1, j, k}^{\imath, n+1}+\left[1+\Delta \tau\left(\gamma_{i}^{\imath}+\left(\bar{r}+\sum_{\substack{\jmath=1 \\
\jmath \neq \imath}}^{N} \lambda_{\imath \jmath}^{Q}\right)\right)\right] V_{i, j, k}^{\imath, n+1} \\
=\sup _{\zeta_{i, j, k}^{\imath, n+1} \in Z_{k}} V_{i, \hat{j}, \hat{k}}^{\imath, n}+\Delta \tau H\left(r_{k}, w_{j}\right) q\left(r_{k}, h\left(w_{j}\right)\right)\left(P_{i}-c\right)+\Delta \tau \sum_{\substack{\jmath=1 \\
\jmath \neq \imath}}^{N} \lambda_{\imath \jmath}^{Q} \chi\left(P_{i} \bar{\xi}^{\imath \jmath}\left(P_{i}\right), V_{i_{\jmath}^{\prime}, j, k}^{\jmath, n+1}, V_{i_{\jmath}^{*}+1, j, k}^{\jmath, n+1}\right) \\
\text { if } P_{i}=P^{\max }
\end{array}
$$

\section{A.1.4 Solving the Local Optimization Problems}

We use the method suggested by Chen and Forsyth [2008] to solve a discrete local optimization problem

$$
\sup _{\zeta_{i, j, k}^{\imath, n+1} \in Z_{k}} V_{i, \hat{j}, \hat{k}}^{\imath, n}
$$

at a mesh node $\left(P_{i}, w_{j}, r_{k}, \tau^{n+1}\right)$ in scheme (A.17). Given a mesh node $\left(P_{i}, w_{j}, r_{k}, \tau^{n+1}\right)$, $w_{\hat{j}}^{n}$ is fixed in (A.15) and in (A.16) $r_{\hat{k}}^{n}$ varies for different values of $\zeta_{i, j, k}^{i, n+1}$, where all the values of $r_{\hat{k}}^{n}$ form a closed region, denoted by

$$
R_{k}=\left\{r_{\hat{k}}^{n} \mid r_{\hat{k}}^{n}=r_{k}+\zeta_{i, j, k}^{\imath, n+1} \Delta \tau, \forall \zeta_{i, j, k}^{i, n+1} \in Z_{k}\right\}
$$

From $R_{k}$ we select a sequence of values, denoted by $\hat{R}_{k}$, which includes all the discrete grid nodes in the $r$ direction and the maximum and minimum values in $R_{k}$. Then we choose the $r_{\hat{k}}^{n} \in \hat{R}_{k}$ which maximizes $V_{i, \hat{j}, \hat{k}}^{i, n}$. This means we now solve an alternative problem

$$
\sup _{r_{\hat{k}}^{n} \in \hat{R}_{k}} V_{i, \hat{j}, \hat{k}}^{i, n}
$$

It is shown in Chen and Forsyth [2008] that the solutions to problems (A.21) and (A.22) 
are consistent.

\section{A.1.5 Matrix Form of the Discrete Equations}

We now set up the matrix form of the discrete Equation (A.17). Let

$$
V_{j, k}^{\imath, n+1}=\left[\begin{array}{c}
V_{0, j, k}^{\imath, n+1} \\
V_{1, j, k}^{\imath, n+1} \\
\vdots \\
V_{i_{\max }^{\imath, j, k}, k}^{\imath+1}
\end{array}\right]
$$

and

$$
V_{\hat{j}, \hat{k}}^{\imath, n}=\left[\begin{array}{c}
V_{0, \hat{j}, \hat{k}}^{\imath, n} \\
V_{1, \hat{j}, \hat{k}}^{i, n} \\
\vdots \\
V_{i_{\max }, \hat{j}, \hat{k}}^{\imath, n}
\end{array}\right]
$$

Let

$$
\bar{V}_{i, \hat{j}, \hat{k}}^{\imath, n}=\sup _{\zeta_{i, j, k}^{\imath, n+1} \in Z_{k}} V_{i, \hat{j}, \hat{k}}^{\imath, n}
$$

Then we have

$$
\bar{V}_{\hat{j}, \hat{k}}^{\imath, n}=\left[\begin{array}{c}
\bar{V}_{0, \hat{j}, \hat{k}}^{\imath, n} \\
\bar{V}_{1, \hat{j}, \hat{k}}^{i, n} \\
\vdots \\
\bar{V}_{i_{\max }^{\imath, n}, \hat{j}, \hat{k}}
\end{array}\right]
$$

Let

$$
\bar{H}_{j, k}=H\left(r_{k}, w_{j}\right) q\left(r_{k}, h\left(w_{j}\right)\right) .
$$


The discrete Equation (A.17) may be written in matrix form as

$$
\left[I+M^{\imath}\right] V_{j, k}^{\imath, n+1}=\bar{V}_{\hat{j}, \hat{k}}^{\imath, n}+\Delta \tau \bar{H}_{j, k}(P-\iota c)+\Delta \tau \Xi\left(V_{j, k}^{\imath, n+1}\right) .
$$

where

$$
\begin{gathered}
{\left[M^{\imath} V_{j, k}^{\imath, n+1}\right]_{\text {ith row }}=\Delta \tau\left[-\gamma_{i}^{\imath} V_{i-1, j, k}^{\imath, n+1}+\left(\gamma_{i}^{\imath}+\beta_{i}^{\imath}+\left(\bar{r}+\sum_{\substack{\jmath=1 \\
\jmath \neq \imath}}^{N} \lambda_{\imath \jmath}^{Q}\right)\right) V_{i, j, k}^{i, n+1}-\beta_{i}^{\imath} V_{i+1, j, k}^{\imath, n+1}\right]} \\
I=\left[\begin{array}{cccc}
1 & 0 & \cdots & 0 \\
0 & 1 & \cdots & 0 \\
\vdots & \vdots & \ddots & \vdots \\
0 & 0 & \cdots & 1
\end{array}\right] \\
\iota=\left[\begin{array}{c}
1 \\
1 \\
\vdots \\
1
\end{array}\right]
\end{gathered}
$$

and

$$
\Xi\left(V_{j, k}^{i, n+1}\right)=\left[\begin{array}{llllll}
\tilde{V}_{j, k}^{1, n+1} & \tilde{V}_{j, k}^{2, n+1} & \cdots & \tilde{V}_{j, k}^{, n+n+1} & \cdots & \tilde{V}_{j, k}^{N, n+1}
\end{array}\right]\left[\begin{array}{c}
\lambda_{\imath 1}^{Q} \\
\lambda_{\imath 2}^{Q} \\
\vdots \\
0 \\
\vdots \\
\lambda_{\imath N}^{Q}
\end{array}\right]
$$

where 


$$
\tilde{V}_{j, k}^{\jmath, n+1}=\left[\begin{array}{c}
\tilde{V}_{0, j, k}^{\jmath, n+1} \\
\tilde{V}_{1, j, k}^{\jmath, n+1} \\
\vdots \\
\tilde{V}_{i, j, k}^{\jmath, n+1} \\
\vdots \\
\tilde{V}_{i_{\max }^{\jmath, j, k}}^{\jmath, n+1}
\end{array}\right]
$$

and 0 is the $\imath$ th element of the column vector. The $i$ th element of vector $\tilde{V}_{j, k}^{j, n+1}$ for $\jmath=1, \ldots, N$ is given by $\chi\left(P_{i} \bar{\xi}^{\imath \jmath}\left(P_{i}\right), V_{i_{\jmath}^{*}, j, k}^{\jmath, n+1}, V_{i_{\jmath}^{*}+1, j, k}^{\jmath, n+1}\right)$.

\section{A.1.6 Solution Algorithms}

To solve Equation (A.23), we use the fixed point iteration algorithm as described in Kennedy [2007]. The algorithm is given by

$$
\begin{aligned}
& V^{0}=\text { Option Payoff } \\
& \text { for } n=0, \ldots, \text { do } \\
& \text { for } j=0, \ldots, \text { do } \\
& \text { for } k=0, \ldots, \text { do } \\
& \text { for } \imath=0, \ldots, \text { do } \\
& \text { for } i=0, \ldots, \text { do } \\
& \bar{V}_{i, \hat{j}, \hat{k}}^{\imath, n}=\sup _{\zeta_{i, j, k}^{\imath, n+1} \in Z_{k}} V_{i, \hat{j}, \hat{k}}^{\imath, n} \\
& \text { end for } \\
& \text { end for } \\
& \text { for } j=0, \ldots, \text { do } \\
& \text { for } k=0, \ldots, \text { do } \\
& \text { for } \imath=0, \ldots, \text { do } \\
& V_{j, k}^{\imath, n+1,0}=V_{j, k}^{\imath, n} \\
& \text { end for } \\
& \text { end for }
\end{aligned}
$$




\section{end for}

$s=0$

while Error $>$ Tol do

for $\imath=0, \ldots$, do

Solve

$$
\left[I+M^{\imath}\right] V_{j, k}^{\imath, n+1, s+1}=\bar{V}_{\hat{j}, \hat{k}}^{\imath, n}+\Delta \tau \bar{H}_{j, k}(P-\iota c)+\Delta \tau \Xi\left(V_{j, k}^{\imath, n+1, s}\right)
$$

\section{end for}

$$
\begin{aligned}
& \text { Error }=\max _{\imath}\left\{\max _{i}\left(\frac{\left|V_{i, j, k}^{\imath, n+1, s+1}-V_{i, j, k}^{\imath, n+1, s}\right|}{\max \left(1,\left|V_{i, j, k}^{\imath, n+1, s}\right|,\left|V_{i, j, k}^{\imath, n}\right|\right)}\right)\right\} \\
& s=s+1
\end{aligned}
$$

\section{end while}

\section{end for}

\section{end for}

\section{end for}

Theorem 1. (Convergence of the Fixed Point Iteration) Assuming the following conditions are satisfied: $\gamma_{i}^{\imath} \geq 0 ; \beta_{i}^{\imath} \geq 0 ; \bar{r} \geq 0 ; \lambda_{\imath \jmath}^{Q} \geq 0$, then the fixed point iteration is globally convergent, and the maximum error at each iteration satisfies

$$
\left\|\vartheta^{s+1}\right\|_{\infty} \leq\left\|\vartheta^{s}\right\|_{\infty} \frac{\sum_{\substack{\jmath=1 \\ \jmath \neq \imath^{\star}}}^{N} \lambda_{\imath^{\star} \jmath}^{Q} \Delta \tau}{1+\left(\bar{r}+\sum_{\substack{\jmath=1 \\ \jmath \neq \imath^{\star}}}^{N} \lambda_{\imath^{\star} \jmath}^{Q}\right) \Delta \tau} .
$$

Proof. Let $\vartheta_{i, j, k}^{\imath, s}$ be the error between the exact solution $V_{i, j, k}^{\imath, n+1}$ and the $s$ th guess $V_{i, j, k}^{\imath, n+1, s}$ for the sth iteration. This could be written as

$$
\vartheta_{i, j, k}^{\imath, s}=V_{i, j, k}^{\imath, n+1}-V_{i, j, k}^{\imath, n+1, s}
$$

Use the fixed point iteration above and Equation (A.23), we obtain

$$
\left[I+M^{\imath}\right] \vartheta_{j, k}^{\imath, s+1}=\Delta \tau \Xi\left(\vartheta_{j, k}^{\imath, s}\right)
$$

in matrix form. For the $i$ th row we have 


$$
\begin{aligned}
& {\left[1+\Delta \tau\left(\gamma_{i}^{\imath}+\beta_{i}^{\imath}+\left(\bar{r}+\sum_{\substack{\jmath=1 \\
\jmath \neq \imath}}^{N} \lambda_{\imath \jmath}^{Q}\right)\right)\right] \vartheta_{i, j, k}^{\imath, s+1}} \\
& =\Delta \tau \gamma_{i}^{\imath} \vartheta_{i-1, j, k}^{\imath, s+1}+\Delta \tau \beta_{i}^{\imath} \vartheta_{i+1, j, k}^{\imath, s+1}+\Delta \tau \sum_{\substack{\jmath=1 \\
\jmath \neq \imath}}^{N} \lambda_{\imath \jmath}^{Q} \chi\left(P_{i} \bar{\xi}^{\imath \jmath}\left(P_{i}\right), \vartheta_{i_{\jmath}^{*}, j, k}^{\jmath, s}, \vartheta_{i_{\jmath}^{*}+1, j, k}^{\jmath, s}\right)
\end{aligned}
$$

Let, $\left\|\vartheta_{j, k}^{\imath, s}\right\|_{\infty}=\max _{i}\left|\vartheta_{i, j, k}^{\imath, s}\right|$. Since $\chi$ is a linear operator and $\gamma_{i}^{\imath} \geq 0 ; \beta_{i}^{\imath} \geq 0 ; \lambda_{\imath \jmath}^{Q} \geq 0$, we get

$$
\begin{aligned}
& {\left[1+\Delta \tau\left(\gamma_{i}^{\imath}+\beta_{i}^{\imath}+\left(\bar{r}+\sum_{\substack{\jmath=1 \\
\jmath \neq \imath}}^{N} \lambda_{\imath \jmath}^{Q}\right)\right)\right]\left|\vartheta_{i, j, k}^{\imath, s+1}\right|} \\
& \leq \Delta \tau \gamma_{i}^{\imath}\left\|\vartheta_{j, k}^{\imath, s+1}\right\|_{\infty}+\Delta \tau \beta_{i}^{\imath}\left\|\vartheta_{j, k}^{\imath, s+1}\right\|_{\infty}+\Delta \tau \sum_{\substack{\jmath=1 \\
\jmath \neq \imath}}^{N} \lambda_{\imath \jmath}^{Q}\left\|\vartheta_{j, k}^{\jmath, s}\right\|_{\infty}
\end{aligned}
$$

Let, $\left\|\vartheta_{j, k}^{s}\right\|_{\infty}=\max _{\imath}\left\|\vartheta_{j, k}^{\imath, s}\right\|_{\infty}$. We can further write

$$
\begin{aligned}
& {\left[1+\Delta \tau\left(\gamma_{i}^{\imath}+\beta_{i}^{\imath}+\left(\bar{r}+\sum_{\substack{\jmath=1 \\
\jmath \neq \imath}}^{N} \lambda_{\imath \jmath}^{Q}\right)\right)\right]\left|\vartheta_{i, j, k}^{\imath, s+1}\right|} \\
& \leq \Delta \tau \gamma_{i}^{\imath}\left\|\vartheta_{j, k}^{s+1}\right\|_{\infty}+\Delta \tau \beta_{i}^{\imath}\left\|\vartheta_{j, k}^{s+1}\right\|_{\infty}+\Delta \tau \sum_{\substack{j=1 \\
j \neq \imath}}^{N} \lambda_{\imath \jmath}^{Q}\left\|\vartheta_{j, k}^{s}\right\|_{\infty}
\end{aligned}
$$

Similarly, let $\left\|\vartheta_{k}^{s}\right\|_{\infty}=\max _{j}\left\|\vartheta_{j, k}^{s}\right\|_{\infty}$ and $\left\|\vartheta^{s}\right\|_{\infty}=\max _{k}\left\|\vartheta_{k}^{s}\right\|_{\infty}$. We get

$$
\begin{aligned}
& {\left[1+\Delta \tau\left(\gamma_{i}^{\imath}+\beta_{i}^{\imath}+\left(\bar{r}+\sum_{\substack{j=1 \\
\jmath \neq \imath}}^{N} \lambda_{\imath \jmath}^{Q}\right)\right)\right]\left|\vartheta_{i, j, k}^{\imath, s+1}\right|} \\
& \leq \Delta \tau \gamma_{i}^{\imath}\left\|\vartheta^{s+1}\right\|_{\infty}+\Delta \tau \beta_{i}^{\imath}\left\|\vartheta^{s+1}\right\|_{\infty}+\Delta \tau \sum_{\substack{\jmath=1 \\
\jmath \neq \imath}}^{N} \lambda_{\imath \jmath}^{Q}\left\|\vartheta^{s}\right\|_{\infty}
\end{aligned}
$$


This equation holds for all $\imath, i, j, k$. It is also true for $\imath^{\star}, i^{\star}, j^{\star}, k^{\star}$ such that $\left|\vartheta_{i^{\star}, j^{\star}, k^{\star}}^{\imath^{\star}, s+1}\right|=$ $\left\|\vartheta^{s+1}\right\|_{\infty}$. Now,

$$
\begin{aligned}
& {\left[1+\Delta \tau\left(\gamma_{i^{\star}}^{\imath^{\star}}+\beta_{i^{\star}}^{\imath^{\star}}+\left(\bar{r}+\sum_{\substack{j=1 \\
j \neq \imath^{\star}}}^{N} \lambda_{i^{\star} \jmath}^{Q}\right)\right)\right]\left\|\vartheta^{s+1}\right\|_{\infty}} \\
& \leq \Delta \tau \gamma_{i^{\star}}^{\imath^{\star}}\left\|\vartheta^{s+1}\right\|_{\infty}+\Delta \tau \beta_{i^{\star}}^{2^{\star}}\left\|\vartheta^{s+1}\right\|_{\infty}+\Delta \tau \sum_{\substack{\jmath=1 \\
j \neq \imath^{\star}}}^{N} \lambda_{i^{\star} \jmath}^{Q}\left\|\vartheta^{s}\right\|_{\infty} .
\end{aligned}
$$

This reduces to

$$
\left\|\vartheta^{s+1}\right\|_{\infty} \leq\left\|\vartheta^{s}\right\|_{\infty} \frac{\sum_{\substack{\jmath=1 \\ j \neq \imath^{\star}}}^{N} \lambda_{l^{\star} \jmath}^{Q} \Delta \tau}{1+\left(\bar{r}+\sum_{\substack{j=1 \\ j \neq \imath^{\star}}}^{N} \lambda_{\imath^{\star} \jmath}^{Q}\right) \Delta \tau}<\left\|\vartheta^{s}\right\|_{\infty}
$$

\section{A.1.7 Derivation of Equation (A.10) for Interior Prices}

We now derive

$$
\left(C_{\varepsilon} V\right)_{i, j, k}^{\imath, n}=\gamma_{i}^{\imath} V_{i-1, j, k}^{\imath, n}+\beta_{i}^{\imath} V_{i+1, j, k}^{\imath, n}-\left(\gamma_{i}^{\imath}+\beta_{i}^{\imath}+\left(\bar{r}+\sum_{\substack{\jmath=1 \\ \jmath \neq \imath}}^{N} \lambda_{\imath \jmath}^{Q}\right)\right) V_{i, j, k}^{\imath, n} \text { if } P_{i} \in\left(0, P^{\max }\right)
$$

Discretize the following equation and apply the second order central differencing in the first and second order derivative terms.

$$
C V^{\imath}=\frac{1}{2}\left(\sigma^{\imath}\right)^{2} P^{2} \frac{\partial^{2} V^{\imath}}{\partial P^{2}}+\left[\alpha^{\imath}\left(K^{\imath}-P\right)-\Lambda^{\imath} \sigma^{\imath} P\right] \frac{\partial V^{\imath}}{\partial P}-\left(\bar{r}+\sum_{\substack{\jmath=1 \\ \jmath \neq \imath}}^{N} \lambda_{\imath \jmath}^{Q}\right) V^{\imath} .
$$

Now we have 


$$
\begin{aligned}
& \left(C_{\varepsilon} V\right)_{i, j, k}^{\imath, n}=\frac{1}{2}\left(\sigma^{\imath}\right)^{2} P_{i}^{2}\left[\frac{\frac{V_{i+1, j, k}^{\imath, n}-V_{i, j, k}^{\imath, n}}{P_{i+1}-P_{i}}-\frac{V_{i, j, k}^{\imath, n}-V_{i-1, j, k}^{\imath, n}}{P_{i}-P_{i-1}}}{\frac{P_{i+1}-P_{i}+P_{i}-P_{i-1}}{2}}\right] \\
& +\left[\alpha^{\imath}\left(K^{\imath}-P_{i}\right)-\Lambda^{\imath} \sigma^{\imath} P_{i}\right] \frac{V_{i+1, j, k}^{\imath, n}-V_{i-1, j, k}^{\imath, n}}{P_{i+1}-P_{i}+P_{i}-P_{i-1}}-\left(\bar{r}+\sum_{\substack{\jmath=1 \\
j \neq \imath}}^{N} \lambda_{\imath \jmath}^{Q}\right) V_{i, j, k}^{\imath, n} \\
& =\left[\frac{\left(\sigma^{\imath}\right)^{2} P_{i}^{2}}{\left(P_{i}-P_{i-1}\right)\left(P_{i+1}-P_{i-1}\right)}-\frac{\alpha^{\imath}\left(K^{\imath}-P_{i}\right)-\Lambda^{\imath} \sigma^{\imath} P_{i}}{P_{i+1}-P_{i-1}}\right] V_{i-1, j, k}^{\imath, n} \\
& +\left[\frac{\left(\sigma^{\imath}\right)^{2} P_{i}^{2}}{\left(P_{i+1}-P_{i}\right)\left(P_{i+1}-P_{i-1}\right)}+\frac{\alpha^{\imath}\left(K^{\imath}-P_{i}\right)-\Lambda^{\imath} \sigma^{\imath} P_{i}}{P_{i+1}-P_{i-1}}\right] V_{i+1, j, k}^{\imath, n} \\
& -\left[\left[\frac{\left(\sigma^{\imath}\right)^{2} P_{i}^{2}}{\left(P_{i}-P_{i-1}\right)\left(P_{i+1}-P_{i-1}\right)}-\frac{\alpha^{\imath}\left(K^{\imath}-P_{i}\right)-\Lambda^{\imath} \sigma^{\imath} P_{i}}{P_{i+1}-P_{i-1}}\right]\right. \\
& \left.+\left[\frac{\left(\sigma^{\imath}\right)^{2} P_{i}^{2}}{\left(P_{i+1}-P_{i}\right)\left(P_{i+1}-P_{i-1}\right)}+\frac{\alpha^{\imath}\left(K^{\imath}-P_{i}\right)-\Lambda^{\imath} \sigma^{\imath} P_{i}}{P_{i+1}-P_{i-1}}\right]+\left(\bar{r}+\sum_{\substack{\jmath=1 \\
j \neq \imath}}^{N} \lambda_{\imath \jmath}^{Q}\right)\right] V_{i, j, k}^{\imath, n} \\
& =\gamma_{i, \text { central }}^{\imath} V_{i-1, j, k}^{\imath, n}+\beta_{i, \text { central }}^{\imath} V_{i+1, j, k}^{\imath, n}-\left(\gamma_{i, \text { central }}^{\imath}+\beta_{i, \text { central }}^{\imath}+\left(\bar{r}+\sum_{\substack{j=1 \\
j \neq \imath}}^{N} \lambda_{\imath \jmath}^{Q}\right)\right) V_{i, j, k}^{\imath, n} \\
& \text { if } P_{i} \in\left(0, P^{\max }\right)
\end{aligned}
$$

where, $\gamma_{i, \text { central }}^{\imath}$ and $\beta_{i, \text { central }}^{\imath}$ are defined by

$$
\begin{gathered}
\gamma_{i, \text { central }}^{\imath}=\frac{\left(\sigma^{\imath}\right)^{2} P_{i}^{2}}{\left(P_{i}-P_{i-1}\right)\left(P_{i+1}-P_{i-1}\right)}-\frac{\alpha^{\imath}\left(K^{\imath}-P_{i}\right)-\Lambda^{\imath} \sigma^{\imath} P_{i}}{P_{i+1}-P_{i-1}} \\
\beta_{i, \text { central }}^{\imath}=\frac{\left(\sigma^{\imath}\right)^{2} P_{i}^{2}}{\left(P_{i+1}-P_{i}\right)\left(P_{i+1}-P_{i-1}\right)}+\frac{\alpha^{\imath}\left(K^{\imath}-P_{i}\right)-\Lambda^{\imath} \sigma^{\imath} P_{i}}{P_{i+1}-P_{i-1}}, i=1, \ldots, i_{\max }-1 .
\end{gathered}
$$

If either $\gamma_{i \text {,central }}^{\imath}$ or $\beta_{i, \text { central }}^{\imath}$ is negative, the discrete scheme will not be monotone. In this case we need to use first order forward or backward differencing in the first order 
derivative term.

Applying the forward differencing to $C V^{\imath}$, we have

$$
\begin{aligned}
& \left(C_{\varepsilon} V\right)_{i, j, k}^{\imath, n}=\frac{1}{2}\left(\sigma^{\imath}\right)^{2} P_{i}^{2}\left[\frac{\frac{V_{i+1, j, k}^{\imath, n}-V_{i, j, k}^{\imath, n}}{P_{i+1}-P_{i}}-\frac{V_{i, j, k}^{\imath, n}-V_{i-1, j, k}^{\imath, n}}{P_{i}-P_{i-1}}}{\frac{P_{i+1}-P_{i}+P_{i}-P_{i-1}}{2}}\right] \\
& +\left[\alpha^{\imath}\left(K^{\imath}-P_{i}\right)-\Lambda^{\imath} \sigma^{\imath} P_{i}\right] \frac{V_{i+1, j, k}^{\imath, n}-V_{i, j, k}^{\imath, n}}{P_{i+1}-P_{i}}-\left(\bar{r}+\sum_{\substack{\jmath=1 \\
\jmath \neq \imath}}^{N} \lambda_{\imath \jmath}^{Q}\right) V_{i, j, k}^{\imath, n} \\
& =\left[\frac{\left(\sigma^{\imath}\right)^{2} P_{i}^{2}}{\left(P_{i}-P_{i-1}\right)\left(P_{i+1}-P_{i-1}\right)}\right] V_{i-1, j, k}^{\imath, n}+\left[\frac{\left(\sigma^{\imath}\right)^{2} P_{i}^{2}}{\left(P_{i+1}-P_{i}\right)\left(P_{i+1}-P_{i-1}\right)}\right. \\
& \left.+\frac{\alpha^{\imath}\left(K^{\imath}-P_{i}\right)-\Lambda^{\imath} \sigma^{\imath} P_{i}}{P_{i+1}-P_{i}}\right] V_{i+1, j, k}^{\imath, n}-\left[\left[\frac{\left(\sigma^{\imath}\right)^{2} P_{i}^{2}}{\left(P_{i}-P_{i-1}\right)\left(P_{i+1}-P_{i-1}\right)}\right]\right. \\
& \left.+\left[\frac{\left(\sigma^{\imath}\right)^{2} P_{i}^{2}}{\left(P_{i+1}-P_{i}\right)\left(P_{i+1}-P_{i-1}\right)}+\frac{\alpha^{\imath}\left(K^{\imath}-P_{i}\right)-\Lambda^{\imath} \sigma^{\imath} P_{i}}{P_{i+1}-P_{i}}\right]+\left(\bar{r}+\sum_{\substack{\jmath=1 \\
\jmath \neq \imath}}^{N} \lambda_{\imath \jmath}^{Q}\right)\right] V_{i, j, k}^{\imath, n} \\
& =\gamma_{i, \text { forward }}^{\imath} V_{i-1, j, k}^{\imath, n}+\beta_{i, \text { forward }}^{\imath} V_{i+1, j, k}^{\imath, n}-\left(\gamma_{i, \text { forward }}^{\imath}+\beta_{i, \text { forward }}^{\imath}+\left(\bar{r}+\sum_{\substack{\jmath=1 \\
j \neq \imath}}^{N} \lambda_{\imath \jmath}^{Q}\right)\right) V_{i, j, k}^{\imath, n} \\
& \text { if } P_{i} \in\left(0, P^{\max }\right)
\end{aligned}
$$

where, $\gamma_{i \text {,forward }}^{\imath}$ and $\beta_{i \text {,forward }}^{\imath}$ are defined by

$$
\begin{gathered}
\gamma_{i, \text { forward }}^{\imath}=\frac{\left(\sigma^{\imath}\right)^{2} P_{i}^{2}}{\left(P_{i}-P_{i-1}\right)\left(P_{i+1}-P_{i-1}\right)} \\
\beta_{i, \text { forward }}^{\imath}=\frac{\left(\sigma^{\imath}\right)^{2} P_{i}^{2}}{\left(P_{i+1}-P_{i}\right)\left(P_{i+1}-P_{i-1}\right)}+\frac{\alpha^{\imath}\left(K^{\imath}-P_{i}\right)-\Lambda^{\imath} \sigma^{\imath} P_{i}}{P_{i+1}-P_{i}}, i=1, \ldots, i_{\max }-1 .
\end{gathered}
$$

Applying the backward differencing to $C V^{\imath}$, we have 


$$
\begin{aligned}
\left(C_{\varepsilon} V\right)_{i, j, k}^{\imath, n}= & \frac{1}{2}\left(\sigma^{\imath}\right)^{2} P_{i}^{2}\left[\frac{\frac{V_{i+1, j, k}^{\imath, n}-V_{i, j, k}^{\imath, n}}{P_{i+1}-P_{i}}-\frac{V_{i, j, k}^{\imath, n}-V_{i-1, j, k}^{\imath, n}}{P_{i}-P_{i-1}}}{\frac{P_{i+1}-P_{i}+P_{i}-P_{i-1}}{2}}\right] \\
& +\left[\alpha^{\imath}\left(K^{\imath}-P_{i}\right)-\Lambda^{\imath} \sigma^{\imath} P_{i}\right] \frac{V_{i, j, k}^{\imath, n}-V_{i-1, j, k}^{\imath, n}}{P_{i}-P_{i-1}}-\left(\bar{r}+\sum_{\substack{\jmath=1 \\
j \neq \imath}}^{N} \lambda_{\imath \jmath}^{Q}\right) V_{i, j, k}^{\imath, n} \\
= & {\left[\frac{\left(\sigma^{\imath}\right)^{2} P_{i}^{2}}{\left(P_{i}-P_{i-1}\right)\left(P_{i+1}-P_{i-1}\right)}-\frac{\alpha^{\imath}\left(K^{\imath}-P_{i}\right)-\Lambda^{\imath} \sigma^{\imath} P_{i}}{P_{i}-P_{i-1}}\right] V_{i-1, j, k}^{\imath, n} } \\
& +\left[\frac{\left(\sigma^{\imath}\right)^{2} P_{i}^{2}}{\left(P_{i+1}-P_{i}\right)\left(P_{i+1}-P_{i-1}\right)}\right] V_{i+1, j, k}^{\imath, n}-\left[\left[\frac{\left(\sigma^{\imath}\right)^{2} P_{i}^{2}}{\left(P_{i}-P_{i-1}\right)\left(P_{i+1}-P_{i-1}\right)}\right.\right. \\
& \left.\left.-\frac{\alpha^{\imath}\left(K^{\imath}-P_{i}\right)-\Lambda^{\imath} \sigma^{\imath} P_{i}}{P_{i}-P_{i-1}}\right]+\left[\frac{\sigma^{2} P_{i}^{2}}{\left(P_{i+1}-P_{i}\right)\left(P_{i+1}-P_{i-1}\right)}\right]+\left(\bar{r}+\sum_{\substack{j=1 \\
j \neq \imath}}^{N} \lambda_{\imath \jmath}^{Q}\right)\right] V_{i, j, k}^{\imath, n} \\
= & \gamma_{i, \text { backward }}^{\imath} V_{i-1, j, k}^{\imath, n}+\beta_{i, \text { backward }}^{\imath} V_{i+1, j, k}^{\imath, n}-\left(\gamma_{i, \text { backward }}^{\imath}+\beta_{i, \text { backward }}^{\imath}+\left(\bar{r}+\sum_{\substack{j=1 \\
j \neq \imath}}^{N} \lambda_{\imath \jmath}^{Q}\right)\right) V_{i, j, k}^{\imath, n} \\
& \text { if } P_{i} \in\left(0, P^{\max }\right)
\end{aligned}
$$

where, $\gamma_{i \text {,backward }}^{\imath}$ and $\beta_{i \text {,backward }}^{\imath}$ are defined by

$$
\begin{gathered}
\gamma_{i, \text { backward }}^{\imath}=\frac{\left(\sigma^{\imath}\right)^{2} P_{i}^{2}}{\left(P_{i}-P_{i-1}\right)\left(P_{i+1}-P_{i-1}\right)}-\frac{\alpha^{\imath}\left(K^{\imath}-P_{i}\right)-\Lambda^{\imath} \sigma^{\imath} P_{i}}{P_{i}-P_{i-1}} \\
\beta_{i, \text { backward }}^{\imath}=\frac{\left(\sigma^{\imath}\right)^{2} P_{i}^{2}}{\left(P_{i+1}-P_{i}\right)\left(P_{i+1}-P_{i-1}\right)}, i=1, \ldots, i_{\max }-1 .
\end{gathered}
$$




\section{A.1.8 Derivation of Equation (A.10) for the Price at the Lower Bound}

We now derive

$$
\left(C_{\varepsilon} V\right)_{i, j, k}^{\imath, n}=\beta_{i}^{\imath} V_{i+1, j, k}^{\imath, n}-\left(\beta_{i}^{\imath}+\left(\bar{r}+\sum_{\substack{j=1 \\ \jmath \neq \imath}}^{N} \lambda_{\imath \jmath}^{Q}\right)\right) V_{i, j, k}^{\imath, n} \text { if } P_{i}=0
$$

To discretize the following equation, for $i=0$ we apply the forward differencing to evaluate the first order derivative term in $C_{0} V^{\imath}$.

$$
C_{0} V^{\imath}=\alpha^{\imath} K^{\imath} \frac{\partial V^{\imath}}{\partial P}-\left(\bar{r}+\sum_{\substack{\jmath=1 \\ \jmath \neq \imath}}^{N} \lambda_{\imath \jmath}^{Q}\right) V^{\imath}
$$

We get

$$
\begin{aligned}
\left(C_{\varepsilon} V\right)_{i, j, k}^{\imath, n} & =\alpha^{\imath} K^{\imath} \frac{V_{i+1, j, k}^{\imath, n}-V_{i, j, k}^{\imath, n}}{P_{i+1}-P_{i}}-\left(\bar{r}+\sum_{\substack{\jmath=1 \\
\jmath \neq \imath}}^{N} \lambda_{\imath \jmath}^{Q}\right) V_{i, j, k}^{\imath, n} \\
& =\frac{\alpha^{\imath} K^{\imath}}{P_{i+1}-P_{i}} V_{i+1, j, k}^{\imath, n}-\left[\frac{\alpha^{\imath} K^{\imath}}{P_{i+1}-P_{i}}+\left(\bar{r}+\sum_{\substack{\jmath=1 \\
\jmath \neq \imath}}^{N} \lambda_{\imath \jmath}^{Q}\right)\right] V_{i, j, k}^{\imath, n} \\
& =\beta_{0}^{\imath} V_{i+1, j, k}^{\imath, n}-\left(\beta_{0}^{\imath}+\left(\bar{r}+\sum_{\substack{\jmath=1 \\
\jmath \neq \imath}}^{N} \lambda_{\imath \jmath}^{Q}\right)\right) V_{i, j, k}^{\imath, n} \text { if } P_{i}=0
\end{aligned}
$$

where, $\gamma_{0}^{\imath}$ and $\beta_{0}^{\imath}$ are defined by

$$
\gamma_{0}^{\imath}=0
$$




$$
\beta_{0}^{\imath}=\frac{\alpha^{\imath} K^{\imath}}{P_{1}-P_{0}}
$$

\section{A.1.9 Derivation of Equation (A.10) for the Price at the Upper Bound}

We now derive

$$
\left(C_{\varepsilon} V\right)_{i, j, k}^{\imath, n}=\gamma_{i}^{\imath} V_{i-1, j, k}^{\imath, n}-\left(\gamma_{i}^{\imath}+\left(\bar{r}+\sum_{\substack{\jmath=1 \\ j \neq \imath}}^{N} \lambda_{\imath \jmath}^{Q}\right)\right) V_{i, j, k}^{\imath, n} \text { if } P_{i}=P^{\max }
$$

To discretize the following equation, for $i=i_{\max }$ we apply the backward differencing to evaluate the first order derivative term in $C_{1} V^{\imath}$.

$$
C_{1} V^{\imath}=\left[\alpha^{\imath}\left(K^{\imath}-P\right)-\Lambda^{\imath} \sigma^{\imath} P\right] \frac{\partial V^{\imath}}{\partial P}-\left(\bar{r}+\sum_{\substack{\jmath=1 \\ \jmath \neq \imath}}^{N} \lambda_{\imath \jmath}^{Q}\right) V^{\imath}
$$

We obtain

$$
\begin{aligned}
\left(C_{\varepsilon} V\right)_{i, j, k}^{\imath, n} & =\left[\alpha^{\imath}\left(K^{\imath}-P_{i}\right)-\Lambda^{\imath} \sigma^{\imath} P_{i}\right] \frac{V_{i, j, k}^{\imath, n}-V_{i-1, j, k}^{\imath, n}}{P_{i}-P_{i-1}}-\left(\bar{r}+\sum_{\substack{\jmath=1 \\
\jmath \neq \imath}}^{N} \lambda_{\imath \jmath}^{Q}\right) V_{i, j, k}^{\imath, n} \\
& =\left[-\frac{\alpha^{\imath}\left(K^{\imath}-P_{i}\right)-\Lambda^{\imath} \sigma^{\imath} P_{i}}{P_{i}-P_{i-1}}\right] V_{i-1, j, k}^{\imath, n}-\left[-\frac{\alpha^{\imath}\left(K^{\imath}-P_{i}\right)-\Lambda^{\imath} \sigma^{\imath} P_{i}}{P_{i}-P_{i-1}}+\left(\bar{r}+\sum_{\substack{\jmath=1 \\
j \neq \imath}}^{N} \lambda_{\imath \jmath}^{Q}\right)\right] V_{i, j, k}^{\imath, n} \\
& =\gamma_{i_{\max }}^{\imath} V_{i-1, j, k}^{\imath, n}-\left(\gamma_{i_{\max }}^{\imath}+\left(\bar{r}+\sum_{\substack{\jmath=1 \\
\jmath \neq \imath}}^{N} \lambda_{\imath \jmath}^{Q}\right)\right) V_{i, j, k}^{\imath, n} \text { if } P_{i}=P^{\max }
\end{aligned}
$$


where, $\gamma_{i_{\max }}^{\imath}$ and $\beta_{i_{\max }}^{\imath}$ are defined by

$$
\begin{gathered}
\gamma_{i_{\max }}^{\imath}=-\frac{\alpha^{\imath}\left(K^{\imath}-P_{i_{\max }}\right)-\Lambda^{\imath} \sigma^{\imath} P_{i_{\max }}}{P_{i_{\max }}-P_{i_{\max }-1}} . \\
\beta_{i_{\max }}=0 .
\end{gathered}
$$

\section{A.2 Tables for the Sensitivity Analyses}


Table A.1: Numerical Results with Different Mean Reverting Rate

\begin{tabular}{|c|c|c|c|c|c|c|}
\hline \multicolumn{7}{|c|}{ Total Profit and Change of Total Profit in Regime 1 at Time 0 When the Initial Price is $40 \mathrm{EUR} / \mathrm{MWh}$} \\
\hline Case & & $\begin{array}{l}\text { No Ramping } \\
\text { Restrictions }\end{array}$ & 5000 (CFS-hr) & 3000 (CFS-hr) & 1000 (CFS-hr) & 250 (CFS-hr) \\
\hline \multirow[t]{2}{*}{$\mathrm{HF}$} & Profit & 1282900 & 1278600 & 1271400 & 1258800 & 1233000 \\
\hline & $\% \mathrm{ch}$ & $\mathrm{N} / \mathrm{A}$ & -0.3 & -0.9 & -1.9 & -3.9 \\
\hline \multirow[t]{2}{*}{ FF } & Profit & 1281700 & 1276400 & 1267300 & 1240400 & 1184000 \\
\hline & $\% \mathrm{ch}$ & $\mathrm{N} / \mathrm{A}$ & -0.4 & -1.1 & -3.2 & -7.6 \\
\hline \multicolumn{7}{|c|}{ Total Profit and Change of Total Profit in Regime 2 at Time 0 When the Initial Price is $80 \mathrm{EUR} / \mathrm{MWh}$} \\
\hline \multirow[t]{2}{*}{ HF } & Profit & 1310000 & 1304800 & 1296400 & 1281000 & 1254000 \\
\hline & \%ch & $\mathrm{N} / \mathrm{A}$ & -0.4 & -1.0 & -2.2 & -4.3 \\
\hline \multirow[t]{2}{*}{ FF } & Profit & 1312900 & 1307700 & 1299000 & 1273400 & 1220000 \\
\hline & $\% \mathrm{ch}$ & $\mathrm{N} / \mathrm{A}$ & -0.4 & -1.1 & -3.0 & -7.1 \\
\hline \multicolumn{7}{|c|}{ Total Profit and Change of Total Profit in Regime 2 at Time 0 When the Initial Price is $160 \mathrm{EUR} / \mathrm{MWh}$} \\
\hline \multirow[t]{2}{*}{$\mathrm{HF}$} & Profit & 1398200 & 1391000 & 1379500 & 1353800 & 1319100 \\
\hline & $\% \mathrm{ch}$ & $\mathrm{N} / \mathrm{A}$ & -0.5 & -1.3 & -3.2 & -5.7 \\
\hline \multirow[t]{2}{*}{ FF } & Profit & 1412300 & 1407400 & 1399000 & 1373900 & 1329500 \\
\hline & \%ch & $\mathrm{N} / \mathrm{A}$ & -0.4 & -0.9 & -2.7 & -5.9 \\
\hline
\end{tabular}

Note: HF means half release rate and full reservoir level; FF means full release rate and full reservoir level. Profit is in Euros and \%ch refers to the percent change in profits compared to the case of no ramping restrictions. 
Table A.2: Numerical Results with Different Transition Probabilities

\begin{tabular}{|c|c|c|c|c|c|c|}
\hline \multicolumn{7}{|c|}{ Total Profit and Change of Total Profit in Regime 1 at Time 0 When the Initial Price is $40 \mathrm{EUR} / \mathrm{MWh}$} \\
\hline Case & & $\begin{array}{l}\text { No Ramping } \\
\text { Restrictions }\end{array}$ & 5000 (CFS-hr) & 3000 (CFS-hr) & 1000 (CFS-hr) & 250 (CFS-hr) \\
\hline \multirow[t]{2}{*}{$\mathrm{HF}$} & Profit & 1434100 & 1427400 & 1416900 & 1394100 & 1357400 \\
\hline & $\% \mathrm{ch}$ & $\mathrm{N} / \mathrm{A}$ & -0.5 & -1.2 & -2.8 & -5.4 \\
\hline \multirow[t]{2}{*}{ FF } & Profit & 1432700 & 1424700 & 1411700 & 1371000 & 1296200 \\
\hline & \%ch & $\mathrm{N} / \mathrm{A}$ & -0.6 & -1.5 & -4.3 & -9.5 \\
\hline \multicolumn{7}{|c|}{ Total Profit and Change of Total Profit in Regime 2 at Time 0 When the Initial Price is $80 \mathrm{EUR} / \mathrm{MWh}$} \\
\hline \multirow[t]{2}{*}{$\mathrm{HF}$} & Profit & 1471000 & 1463500 & 1451900 & 1426100 & 1387800 \\
\hline & \%ch & $\mathrm{N} / \mathrm{A}$ & -0.5 & -1.3 & -3.1 & -5.7 \\
\hline \multirow[t]{2}{*}{ FF } & Profit & 1473400 & 1465800 & 1453600 & 1416000 & 1346800 \\
\hline & $\%$ ch & $\mathrm{N} / \mathrm{A}$ & -0.5 & -1.3 & -3.9 & -8.6 \\
\hline \multicolumn{7}{|c|}{ Total Profit and Change of Total Profit in Regime 2 at Time 0 When the Initial Price is $160 \mathrm{EUR} / \mathrm{MWh}$} \\
\hline \multirow[t]{2}{*}{$\mathrm{HF}$} & Profit & 1602100 & 1592700 & 1578200 & 1540400 & 1491500 \\
\hline & $\% \mathrm{ch}$ & $\mathrm{N} / \mathrm{A}$ & -0.6 & -1.5 & -3.9 & -6.9 \\
\hline \multirow[t]{2}{*}{$\mathrm{FF}$} & Profit & 1614000 & 1606800 & 1595500 & 1563400 & 1514600 \\
\hline & $\% \mathrm{ch}$ & $\mathrm{N} / \mathrm{A}$ & -0.5 & -1.2 & -3.1 & -6.2 \\
\hline
\end{tabular}

Note: HF means half release rate and full reservoir level; FF means full release rate and full reservoir level. Profit is in Euros and \%ch refers to the percent change in profits compared to the case of no ramping restrictions. 
Table A.3: Numerical Results with Different Volatilities

\begin{tabular}{|c|c|c|c|c|c|c|}
\hline \multicolumn{7}{|c|}{ Total Profit and Change of Total Profit in Regime 1 at Time 0 When the Initial Price is $40 \mathrm{EUR} / \mathrm{MWh}$} \\
\hline \multirow[t]{2}{*}{ Case } & & No Ramping & 5000 (CFS-hr) & 3000 (CFS-hr) & 1000 (CFS-hr) & 250 (CFS-hr) \\
\hline & & Restrictions & & & & \\
\hline \multirow[t]{2}{*}{$\mathrm{HF}$} & Profit & 1444500 & 1437900 & 1427400 & 1405900 & 1370700 \\
\hline & $\% \mathrm{ch}$ & N/A & -0.5 & -1.2 & -2.7 & -5.1 \\
\hline \multirow[t]{2}{*}{$\mathrm{FF}$} & Profit & 1443100 & 1435200 & 1422100 & 1382600 & 1308900 \\
\hline & $\% \mathrm{ch}$ & N/A & -0.6 & -1.5 & -4.2 & -9.3 \\
\hline \multicolumn{7}{|c|}{ Total Profit and Change of Total Profit in Regime 2 at Time 0 When the Initial Price is $80 \mathrm{EUR} / \mathrm{MWh}$} \\
\hline \multirow[t]{2}{*}{$\mathrm{HF}$} & Profit & 1491500 & 1484100 & 1472600 & 1447300 & 1408800 \\
\hline & \%ch & N/A & -0.5 & -1.3 & -3.0 & -5.6 \\
\hline \multirow[t]{2}{*}{ FF } & Profit & 1494000 & 1486400 & 1474200 & 1437600 & 1370700 \\
\hline & $\% \mathrm{ch}$ & $\mathrm{N} / \mathrm{A}$ & -0.5 & -1.3 & -3.8 & -8.3 \\
\hline \multicolumn{7}{|c|}{ Total Profit and Change of Total Profit in Regime 2 at Time 0 When the Initial Price is $160 \mathrm{EUR} / \mathrm{MWh}$} \\
\hline \multirow[t]{2}{*}{$\mathrm{HF}$} & Profit & 1641500 & 1632400 & 1618100 & 1580500 & 1531000 \\
\hline & $\% \mathrm{ch}$ & N/A & -0.6 & -1.4 & -3.7 & -6.7 \\
\hline \multirow[t]{2}{*}{ FF } & Profit & 1653100 & 1646300 & 1635800 & 1608600 & 1565400 \\
\hline & $\%$ ch & $\mathrm{N} / \mathrm{A}$ & -0.4 & -1.1 & -2.7 & -5.3 \\
\hline
\end{tabular}

Note: HF means half release rate and full reservoir level; FF means full release rate and full reservoir level. Profit is in Euros and \%ch refers to the percent change in profits compared to the case of no ramping restrictions. 
Table A.4: Numerical Results with Different Base Regime Mean

\begin{tabular}{|c|c|c|c|c|c|c|}
\hline \multicolumn{7}{|c|}{ Total Profit and Change of Total Profit in Regime 1 at Time 0 When the Initial Price is $40 \mathrm{EUR} / \mathrm{MWh}$} \\
\hline Case & & $\begin{array}{l}\text { No Ramping } \\
\text { Restrictions }\end{array}$ & 5000 (CFS-hr) & 3000 (CFS-hr) & 1000 (CFS-hr) & 250 (CFS-hr) \\
\hline \multirow[t]{2}{*}{$\mathrm{HF}$} & Profit & 353240 & 349390 & 344550 & 335130 & 325670 \\
\hline & $\% \mathrm{ch}$ & $\mathrm{N} / \mathrm{A}$ & -1.1 & -2.5 & -5.1 & -7.8 \\
\hline \multirow[t]{2}{*}{ FF } & Profit & 354010 & 350210 & 345400 & 333960 & 327760 \\
\hline & $\% \mathrm{ch}$ & $\mathrm{N} / \mathrm{A}$ & -1.1 & -2.4 & -5.7 & -7.4 \\
\hline \multicolumn{7}{|c|}{ Total Profit and Change of Total Profit in Regime 2 at Time 0 When the Initial Price is $80 \mathrm{EUR} / \mathrm{MWh}$} \\
\hline \multirow[t]{2}{*}{ HF } & Profit & 403830 & 399100 & 392940 & 378800 & 364600 \\
\hline & \%ch & $\mathrm{N} / \mathrm{A}$ & -1.2 & -2.7 & -6.2 & -9.7 \\
\hline \multirow[t]{2}{*}{ FF } & Profit & 409700 & 405900 & 401180 & 390370 & 386960 \\
\hline & $\% \mathrm{ch}$ & $\mathrm{N} / \mathrm{A}$ & -0.9 & -2.1 & -4.7 & -5.6 \\
\hline \multicolumn{7}{|c|}{ Total Profit and Change of Total Profit in Regime 2 at Time 0 When the Initial Price is $160 \mathrm{EUR} / \mathrm{MWh}$} \\
\hline \multirow[t]{2}{*}{$\mathrm{HF}$} & Profit & 540470 & 533690 & 524440 & 497970 & 470150 \\
\hline & $\% \mathrm{ch}$ & $\mathrm{N} / \mathrm{A}$ & -1.3 & -3.0 & -7.9 & -13.0 \\
\hline \multirow[t]{2}{*}{$\mathrm{FF}$} & Profit & 557720 & 554080 & 549920 & 542920 & 542270 \\
\hline & \%ch & $\mathrm{N} / \mathrm{A}$ & -0.7 & -1.4 & -2.7 & -2.8 \\
\hline
\end{tabular}

Note: HF means half release rate and full reservoir level; FF means full release rate and full reservoir level. Profit is in Euros and \%ch refers to the percent change in profits compared to the case of no ramping restrictions. 
Table A.5: Numerical Results with Different Production Cost

\begin{tabular}{|c|c|c|c|c|c|c|}
\hline \multicolumn{7}{|c|}{ Total Profit and Change of Total Profit in Regime 1 at Time 0 When the Initial Price is $40 \mathrm{EUR} / \mathrm{MWh}$} \\
\hline Case & & $\begin{array}{l}\text { No Ramping } \\
\text { Restrictions }\end{array}$ & 5000 (CFS-hr) & 3000 (CFS-hr) & 1000 (CFS-hr) & 250 (CFS-hr) \\
\hline \multirow[t]{2}{*}{$\mathrm{HF}$} & Profit & 1794800 & 1790600 & 1782100 & 1765500 & 1729300 \\
\hline & $\% \mathrm{ch}$ & $\mathrm{N} / \mathrm{A}$ & -0.2 & -0.7 & -1.6 & -3.7 \\
\hline \multirow[t]{2}{*}{ FF } & Profit & 1793400 & 1787700 & 1776500 & 1738400 & 1657900 \\
\hline & $\% \mathrm{ch}$ & $\mathrm{N} / \mathrm{A}$ & -0.3 & -0.9 & -3.1 & -7.6 \\
\hline \multicolumn{7}{|c|}{ Total Profit and Change of Total Profit in Regime 2 at Time 0 When the Initial Price is $80 \mathrm{EUR} / \mathrm{MWh}$} \\
\hline \multirow{2}{*}{$\mathrm{HF}$} & Profit & 1826200 & 1821200 & 1811700 & 1792600 & 1755700 \\
\hline & \%ch & $\mathrm{N} / \mathrm{A}$ & -0.3 & -0.8 & -1.8 & -3.9 \\
\hline \multirow[t]{2}{*}{ FF } & Profit & 1828500 & 1823200 & 1812800 & 1778000 & 1702600 \\
\hline & $\%$ ch & $\mathrm{N} / \mathrm{A}$ & -0.3 & -0.9 & -2.8 & -6.9 \\
\hline \multicolumn{7}{|c|}{ Total Profit and Change of Total Profit in Regime 2 at Time 0 When the Initial Price is $160 \mathrm{EUR} / \mathrm{MWh}$} \\
\hline \multirow[t]{2}{*}{$\mathrm{HF}$} & Profit & 1939800 & 1932900 & 1920600 & 1890600 & 1846000 \\
\hline & $\% \mathrm{ch}$ & $\mathrm{N} / \mathrm{A}$ & -0.4 & -1.0 & -2.5 & -4.8 \\
\hline \multirow[t]{2}{*}{ FF } & Profit & 1951500 & 1946600 & 1937200 & 1908200 & 1852800 \\
\hline & $\% \mathrm{ch}$ & $\mathrm{N} / \mathrm{A}$ & -0.3 & -0.7 & -2.2 & -5.1 \\
\hline
\end{tabular}

Note: HF means half release rate and full reservoir level; FF means full release rate and full reservoir level. Profit is in Euros and \%ch refers to the percent change in profits compared to the case of no ramping restrictions. 
Table A.6: Summary of Results from the Sensitivity Analysis

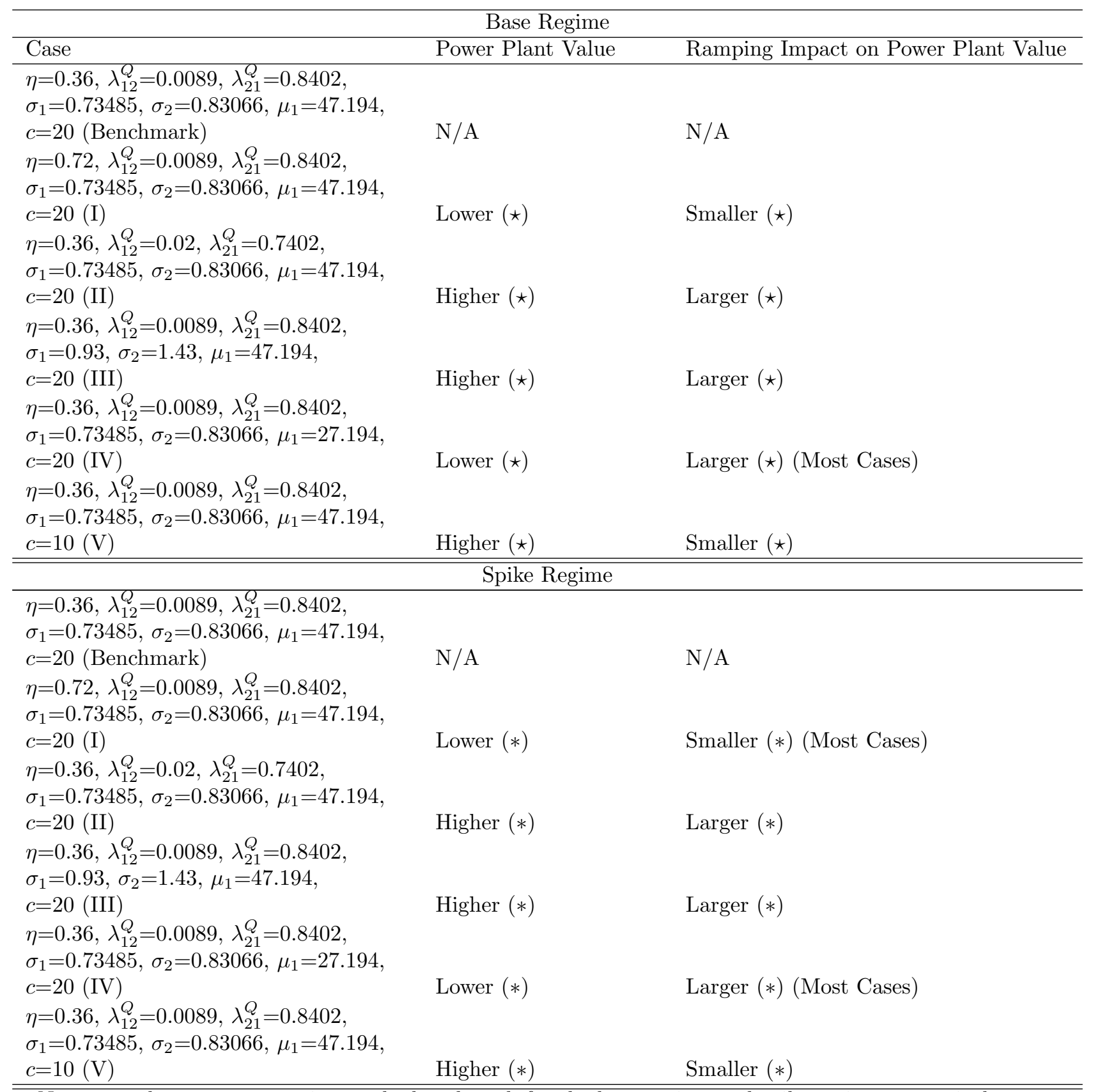

Note: $\star$ and $*$ mean comparing to the benchmark for the base regime and spike regime respectively. Other benchmark parameter values are given in Table 3.3 and the corresponding results are reported in

Table 3.4. 
Table A.7: Base Regime as Single Regime for the Regime Switching Model

\begin{tabular}{|c|c|c|c|c|c|c|}
\hline \multicolumn{7}{|c|}{ Total Profit and Change of Total Profit at Time 0 When the Initial Price is $40 \mathrm{EUR} / \mathrm{MWh}$} \\
\hline Case & & $\begin{array}{l}\text { No Ramping } \\
\text { Restrictions }\end{array}$ & 5000 (CFS-hr) & 3000 (CFS-hr) & 1000 (CFS-hr) & 250 (CFS-hr) \\
\hline \multirow[t]{2}{*}{$\mathrm{HF}$} & Profit & 1329400 & 1325700 & 1318800 & 1307700 & 1282800 \\
\hline & $\% \mathrm{ch}$ & $\mathrm{N} / \mathrm{A}$ & -0.3 & -0.8 & -1.6 & -3.5 \\
\hline \multirow[t]{2}{*}{$\mathrm{FF}$} & Profit & 1328100 & 1323300 & 1314200 & 1286700 & 1228700 \\
\hline & $\%$ ch & $\mathrm{N} / \mathrm{A}$ & -0.4 & -1.1 & -3.1 & -7.5 \\
\hline \multicolumn{7}{|c|}{ Total Profit and Change of Total Profit at Time 0 When the Initial Price is 80 EUR/MWh } \\
\hline \multirow[t]{2}{*}{$\mathrm{HF}$} & Profit & 1363900 & 1359500 & 1351700 & 1338900 & 1315300 \\
\hline & $\%$ ch & $\mathrm{N} / \mathrm{A}$ & -0.3 & -0.9 & -1.8 & -3.6 \\
\hline \multirow[t]{2}{*}{$\mathrm{FF}$} & Profit & 1366100 & 1361900 & 1354300 & 1334700 & 1284400 \\
\hline & $\% \mathrm{ch}$ & $\mathrm{N} / \mathrm{A}$ & -0.3 & -0.9 & -2.3 & -6.0 \\
\hline
\end{tabular}

Note: HF means half release rate and full reservoir level; FF means full release rate and full reservoir level. Profit is in Euros and \%ch refers to the percent change in profits compared to the case of no ramping restrictions.

Table A.8: Base Regime as Single Regime for the Regime Switching Model with Different Mean Reverting Rate

\begin{tabular}{|c|c|c|c|c|c|c|}
\hline \multicolumn{7}{|c|}{ Total Profit and Change of Total Profit at Time 0 When the Initial Price is 40 EUR/MWh } \\
\hline Case & & $\begin{array}{l}\text { No Ramping } \\
\text { Restrictions }\end{array}$ & 5000 (CFS-hr) & 3000 (CFS-hr) & 1000 (CFS-hr) & 250 (CFS-hr) \\
\hline \multirow[t]{2}{*}{$\mathrm{HF}$} & Profit & 1249800 & 1246900 & 1241300 & 1233100 & 1210400 \\
\hline & $\%$ ch & $\mathrm{N} / \mathrm{A}$ & -0.2 & -0.7 & -1.3 & -3.2 \\
\hline \multirow[t]{2}{*}{ FF } & Profit & 1248600 & 1244800 & 1237400 & 1215300 & 1163200 \\
\hline & $\%$ ch & $\mathrm{N} / \mathrm{A}$ & -0.3 & -0.9 & -2.7 & -6.8 \\
\hline \multicolumn{7}{|c|}{ Total Profit and Change of Total Profit at Time 0 When the Initial Price is $80 \mathrm{EUR} / \mathrm{MWh}$} \\
\hline \multirow[t]{2}{*}{ HF } & Profit & 1267900 & 1264500 & 1258100 & 1249000 & 1227100 \\
\hline & \%ch & N/A & -0.3 & -0.8 & -1.5 & -3.2 \\
\hline \multirow[t]{2}{*}{ FF } & Profit & 1270500 & 1267200 & 1260800 & 1242200 & 1192400 \\
\hline & \%ch & $\mathrm{N} / \mathrm{A}$ & -0.3 & -0.8 & -2.2 & -6.2 \\
\hline
\end{tabular}

Note: HF means half release rate and full reservoir level; FF means full release rate and full reservoir level. Profit is in Euros and \%ch refers to the percent change in profits compared to the case of no ramping restrictions. 
Table A.9: Base Regime as Single Regime for the Regime Switching Model with Different Volatilities

\begin{tabular}{|c|c|c|c|c|c|c|}
\hline \multicolumn{7}{|c|}{ Total Profit and Change of Total Profit at Time 0 When the Initial Price is 40 EUR/MWh } \\
\hline \multirow[t]{2}{*}{ Case } & & No Ramping & 5000 (CFS-hr) & 3000 (CFS-hr) & 1000 (CFS-hr) & 250 (CFS-hr) \\
\hline & & Restrictions & & & & \\
\hline \multirow[t]{2}{*}{$\mathrm{HF}$} & Profit & 1379900 & 1374400 & 1365500 & 1351100 & 1324200 \\
\hline & $\% \mathrm{ch}$ & $\mathrm{N} / \mathrm{A}$ & -0.4 & -1.0 & -2.1 & -4.0 \\
\hline \multirow[t]{2}{*}{ FF } & Profit & 1378500 & 1371800 & 1360500 & 1329000 & 1267400 \\
\hline & $\% \mathrm{ch}$ & $\mathrm{N} / \mathrm{A}$ & -0.5 & -1.3 & -3.6 & -8.1 \\
\hline \multicolumn{7}{|c|}{ Total Profit and Change of Total Profit at Time 0 When the Initial Price is 80 EUR/MWh } \\
\hline \multirow[t]{2}{*}{$\mathrm{HF}$} & Profit & 1414700 & 1408400 & 1398500 & 1382500 & 1357000 \\
\hline & $\% \mathrm{ch}$ & $\mathrm{N} / \mathrm{A}$ & -0.5 & -1.2 & -2.3 & -4.1 \\
\hline \multirow[t]{2}{*}{ FF } & Profit & 1416700 & 1410600 & 1400900 & 1377400 & 1323600 \\
\hline & $\% \mathrm{ch}$ & $\mathrm{N} / \mathrm{A}$ & -0.4 & -1.1 & -2.8 & -6.6 \\
\hline
\end{tabular}

Note: HF means half release rate and full reservoir level; FF means full release rate and full reservoir level. Profit is in Euros and \%ch refers to the percent change in profits compared to the case of no ramping restrictions.

Table A.10: Base Regime as Single Regime for the Regime Switching Model with Different Production Cost

\begin{tabular}{|c|c|c|c|c|c|c|}
\hline \multicolumn{7}{|c|}{ Total Profit and Change of Total Profit at Time 0 When the Initial Price is $40 \mathrm{EUR} / \mathrm{MWh}$} \\
\hline Case & & $\begin{array}{l}\text { No Ramping } \\
\text { Restrictions }\end{array}$ & 5000 (CFS-hr) & 3000 (CFS-hr) & 1000 (CFS-hr) & 250 (CFS-hr) \\
\hline $\mathrm{HF}$ & Profit & 1755700 & 1752800 & 1746000 & 1733900 & 1701600 \\
\hline & $\% \mathrm{ch}$ & $\mathrm{N} / \mathrm{A}$ & -0.2 & -0.6 & -1.2 & -3.1 \\
\hline FF & Profit & 1754300 & 1750000 & 1740600 & 1707700 & 1632600 \\
\hline & $\% \mathrm{ch}$ & $\mathrm{N} / \mathrm{A}$ & -0.3 & -0.8 & -2.7 & -6.9 \\
\hline \multicolumn{7}{|c|}{ Total Profit and Change of Total Profit at Time 0 When the Initial Price is $80 \mathrm{EUR} / \mathrm{MWh}$} \\
\hline $\mathrm{HF}$ & Profit & 1789100 & 1785500 & 1777800 & 1764600 & 1734100 \\
\hline \multirow{3}{*}{ FF } & $\%$ ch & $\mathrm{N} / \mathrm{A}$ & -0.2 & -0.6 & -1.4 & -3.1 \\
\hline & Profit & 1791000 & 1787500 & 1779800 & 1755700 & 1688300 \\
\hline & $\%$ ch & N/A & -0.2 & -0.6 & -2.0 & -5.7 \\
\hline
\end{tabular}

Note: HF means half release rate and full reservoir level; FF means full release rate and full reservoir level. Profit is in Euros and \%ch refers to the percent change in profits compared to the case of no ramping restrictions. 


\section{References}

BC Hydro (2005). Shuswap riverwater use plan (shuswap falls and sugar lake project). http://www.ll bc.leg.bc.ca/public/PubDocs/bcdocs/404094/environment33163.pdf.

Bensalem, A., A. Miloudi, S. E. Zouzou, B. Mahdad, and A. Bouhentala (2007). Optimal short term hydro scheduling of large power systems with discretized horizon. Journal of Electrical Engineering 58(4), 214-219.

Benth, F. E., J. Kallsen, and T. Meyer-Brandis (2007). A non-Gaussian OrnsteinUhlenbeck process for electricity spot price modeling and derivatives pricing. Applied Mathematical Finance 14(2), 153-169.

Berland, G., T. Nickelsen, J. Heggenes, F. Okland, E. B. Thorstad, and J. Halleraker (2004). Movements of wild atlantic salmon parr in relation to peaking flows below a hydropower station. River Research and Applications 20(8), 957-966.

Bierbrauer, M., C. Menn, S. T. Rachev, and S. Trueck (2007). Spot and derivative pricing in the EEX power market. Journal of Banking \& Finance 31(11), 3462-3485.

Bradford, M. J., G. C. Taylor, J. A. Allan, and P. A. Higgins (1995). An experimental study of the stranding of juvenile coho salmon and rainbow trout during rapid flow decreases under winter conditions. North American Journal of Fisheries Management 15(2), 473479 . 
Cartea, A. and M. G. Figueroa (2005). Pricing in electricity markets: A mean reverting jump diffusion model with seasonality. Applied Mathematical Finance 12(4), 313-335.

Catalão, J. P. S., S. J. P. S. Mariano, V. M. F. Mendes, and L. A. F. M. Ferreira (2006). Parameterisation effect on the behaviour of a head-dependent hydro chain using a nonlinear model. Electric Power Systems Research 76 (6-7), 404-412.

Chen, S. and M. Insley (2012). Regime switching in stochastic models of commodity prices: An application to an optimal tree harvesting problem. Journal of Economic Dynamics and Control 36(2), 201-219.

Chen, Z. (2008). Numerical methods for optimal stochastic control in finance. Ph.D. thesis, University of Waterloo.

Chen, Z. and P. A. Forsyth (2007). A semi-lagrangian approach for natural gas storage valuation and optimal operation. SIAM Journal on Scientific Computing 30(1), 339-368.

Chen, Z. and P. A. Forsyth (2008). Pricing Hydroelectric Power Plants with/without Operational Restrictions: a Stochastic Control Approach. Nova Science Publishers. In Ehrhardt, M. (eds.), Nonlinear Models in Mathematical Finance. pp. 253-281.

Chen, Z. and P. A. Forsyth (2010). Implications of a regime-switching model on natural gas storage valuation and optimal operation. Quantitative Finance 10(2), 159-176.

Conejo, A. J., J. M. Arroyo, J. Contreras, and F. A. Villamor (2002). Self-scheduling of a hydro producer in a pool-based electricity market. IEEE Transactions on Power Systems 17(4), 1265-1272.

Cross, D. and J. M. DosSantos (1988). Lower flathead system fisheries study. http://pisces.bpa.gov/release/documents/documentviewer.aspx?doc=39830-1A.

Cushman, R. M. (1985). Review of ecological effects of rapidly varying flows downstream from hydroelectric facilities. North American Journal of Fisheries Management 5(3a), 330-339. 
De Jong, C. (2006). The nature of power spikes: A regime-switch approach. Studies in Nonlinear Dynamics \& Econometrics 10(3), Article 3.

Deng, S. (2000). Stochastic models of energy commodity prices and their applications: Mean-reversion with jumps and spikes. Working Peper, University of California Energy Institute.

Deng, S., Y. Shen, and H. Sun (2006). Stochastic co-optimization for hydro-electric power generation. http://www.pserc.wisc.edu/documents/publications/papers/2007-generalpublications/hydropowercooptimdeng.pdf.

Dewees, D. N. (2008). Pollution and the price of power. The Energy Journal 29(2), 81-100.

d'Halluin, Y., P. Forsyth, and G. Labahn (2004). A penalty method for american options with jump diffutions processes. Numerische Mathematik 97, 321-352.

Edwards, B. K. (2003). The Economics of Hydroelectric Power. Edward Elgar Publishing.

Edwards, B. K., S. J. Flaim, and J. D. Ancrile (1992). Maximizing the value of thermally integrated hydroelectric generating facilities. http://www.osti.gov/bridge/servlets/purl/10155080-eyHG0j/10155080.pdf.

Edwards, B. K., S. J. Flaim, and R. E. Howitt (1999). Optimal provision of hydroelectric power under environmental and regulatory constraints. Land Economics 75(2), 267-283.

Escribano, A., J. I. Pena, and P. Villaplana (2011). Modeling electricity prices: International evidence. Oxford Bulletin of Economics and Statistics 73(5), 622-650.

Ethier, R. and T. Mount (1998). Estimating the volatility of spot prices in restructured electricity markets and the implications for option values. Working Paper, Cornell University.

Flathead Lakers (2005). Required minimum flows for the Flathead River below Kerr Dam. http://www.flatheadlakers.org/flatheadlakebasin/lakelevels/minimumflows2.html. 
Flodmark, L. E. W., H. A. Urke, J. H. Halleraker, J. V. Arnekleiv, L. A. Vollestad, and A. B. S. Poleo (2002). Cortisol and glucose responses in juvenile brown trout subjected to a fluctuating flow regime in an artificial stream. Journal of Fish Biology 60(1), 238-248.

Forsyth, P. and K. Vetzal (2014). An optimal stochastic control framework for determining the cost of hedging of variable annuities. Journal of Economic Dynamics and Control 44 , $29-53$.

Freeman, M. C., Z. H. Bowen, K. D. Bovee, and E. R. Irwin (2001). Flow and habitat effects on juvenile fish abundance in natural and altered flow regimes. Ecological Applications 11(1), 179-190.

Geman, H. and A. Roncoroni (2006). Understanding the fine structure of electricity prices. Journal of Business 79(3), 1225-1262.

Gouraud, V., H. Capra, C. Sabaton, L. Tissot, P. Lim, F. Vandewalle, G. Fahrner, and Y. Souchon (2008). Long-term simulations of the dynamics of trout populations on river reaches bypassed by hydroelectric installations - analysis of the impact of different hydrological scenarios. River Research and Applications 24 (9), 1185-1205.

Grand, T. C., S. F. Railsback, J. W. Hayse, and K. E. LaGory (2006). A physical habitat model for predicting the effects of flow fluctuations in nursery habitats of the endangered colorado pikeminnow (Ptychocheilus Lucius). River Research and Applications 22(10), $1125-1142$.

Guan, X., A. Svoboda, and C. Li (1999). Scheduling hydro power systems with restricted operating zones and discharge ramping constraints. IEEE Transactions on Power Systems $14(1), 126-131$.

Harpman, D. A. (1999). Assessing the short-run economic cost of environmental constraints on hydropower operations at Glen Canyon Dam. Land Economics 75(3), 390-401.

Hendry, C. and C. Chang (2001). Investigations of fish communities and habitat in the Abitibi Canyon generating station tailwater. OMNR, Northeast Science and Technology, NEST Information Report IR-026, 42p. + App. 
Heydari, S. and A. Siddiqui (2010). Valuing a gas-fired power plant: A comparison of ordinary linear models, regime-switching approaches, and models with stochastic volatility. Energy Economics 32(3), 709-725.

Hreinsson, E. B. (1988). Optimal short term operation of a purely hydroelectric system. IEEE Transactions on Power Systems 3(3), 1072-1077.

Huisman, R. (2008). The influence of temperature on spike probability in day-ahead power prices. Energy Economics 30(5), 2697-2704.

Huisman, R. and C. De Jong (2003). Option pricing for power prices with spikes. Energy Power Risk Management 7(11), 12-16.

Huppert, D. D. (1999). Snake river salmon recovery: Quantifying the costs. Contemporary Economic Policy 17(4), 476-491.

Hvidsten, N. A. (1985). Mortality of pre-smolt atlantic salmon, Salmo Salar 1., and brown trout, Salmo Trutta l., caused by fluctuating water levels in the regulated river Nidelva, central Norway. Journal of Fish Biology 27(6), 711-718.

Jager, H. I. and M. S. Bevelhimer (2007). How run-of-river operation affects hydropower generation and value. Environmental Management 40(6), 1004-1015.

Jager, H. I. and B. T. Smith (2008). Sustainable reservoir operation: Can we generate hydropower and preserve ecosystem values? River Research and Applications 24(3), 340-352.

Janczura, J. and R. Weron (2009). Regime-switching models for electricity spot prices: Introducing heteroskedastic base regime dynamics and shifted spike distributions. http://mpra.ub.uni-muenchen.de/18784/.

Janczura, J. and R. Weron (2010). An empirical comparison of alternate regime-switching models for electricity spot prices. Energy Economics 32(5), 1059-1073.

Kennedy, J. S. (2007). Hedging contingent claims in markets with jumps. Ph.D. thesis, University of Waterloo. 
Kilgour, B. W., J. Neary, D. Ming, and D. Beach (2005). Preliminary investigations of the use and status of instream-flow-needs methods in Ontario with specific reference to application with hydroelectric developments. Canadian Manuscript Report of Fisheries and Aquatic Sciences 2723: v + 30p.

Kim, C.-J. (1994). Dynamic linear models with markov-switching. Journal of Econometrics $60(1-2), 1-22$.

Kolos, S. P. and E. I. Ronn (2008). Estimating the commodity market price of risk for energy prices. Energy Economics 30(2), 621-641.

Kotchen, M. J., M. R. Moore, F. Lupi, and E. S. Rutherford (2006). Environmental constraints on hydropower: An ex-post benefit-cost analysis of dam relicensing in Michigan. Land Economics 82(3), 384-403.

Lucia, J. J. and E. S. Schwartz (2002). Electricity prices and power derivatives: Evidence from the Nordic power exchange. Review of Derivatives Research 5(1), 5-50.

Marty, J., K. Smokorowski, and M. Power (2009). The influence of fluctuating ramping rates on the food web of boreal rivers. River Research and Applications 25(8), 962-974.

Ministry of Natural Resources (2003). Water management planning guidelines for waterpower, appendix g: Aquatic ecosystem guidelines. Technical report. http://www.owa.ca/pdfs/AEG-Jan2003.pdf.

Misiorek, A., S. Trueck, and R. Weron (2006). Point and interval forecasting of spot electricity prices: Linear vs. non-linear time series models. Studies in Nonlinear Dynamics \& Econometrics 10(3), Article 2.

Mount, T. D., Y. Ning, and X. Cai (2006). Predicting price spikes in electricity markets using a regime-switching model with time-varying parameters. Energy Economics 28(1), $62-80$. 
Murchie, K. J., K. P. Hair, C. E. Pullen, T. D. Redpath, H. R. Stephens, and S. J. Cooke (2008). Fish response to modified flow regimes in regulated rivers: Research methods, effects and opportunities. River Research and Applications 24(2), 197-217.

Niu, S. and M. Insley (2013). On the economics of ramping rate restrictions at hydro power plants: Balancing profitability and environmental costs. Energy Economics 39, 39-52.

Niu, S. and M. Insley (2014). An options pricing approach to ramping rate restrictions at hydro power plants. Working Paper, University of Waterloo.

Oliveira, P., S. McKee, and C. Coles (1993). Optimal scheduling of a hydro thermal power generation system. European Journal of Operational Research 71 (3), 334-340.

Petts, G. E. and I. P. Maddock (1994). Flow Allocation for In-river Needs. Blackwell Science. In Calow, P., and G. E. Petts (eds.), The Rivers Handbook. pp. 289-307.

Philpott, A. B., M. Craddock, and H. Waterer (2000). Hydro-electric unit commitment subject to uncertain demand. European Journal of Operational Research 125(2), 410424.

Rambharat, B. R., A. E. Brockwell, and D. J. Seppi (2005). A threshold autoregressive model for wholesale electricity prices. Journal of the Royal Statistical Society: Series C (Applied Statistics) 54(2), 287-299.

Saltveit, S. J., J. H. Halleraker, J. V. Arnekleiv, and A. Harby (2001). Field experiments on stranding in juvenile atlantic salmon (Salmo Salar) and brown trout (Salmo Trutta) during rapid flow decreases caused by hydropeaking. Regulated Rivers: Research \& Management 17(4-5), 609-622.

Scruton, D. A., L. M. N. Ollerhead, K. D. Clarke, C. Pennell, K. Alfredsen, A. Harby, and D. Kelley (2003). The behavioural response of juvenile atlantic salmon (Salmo Salar) and brook trout (Salvelinus Fontinalis) to experimental hydropeaking on a Newfoundland (Canada) river. River Research and Applications 19(5-6), 577-587. 
Scruton, D. A., C. Pennell, L. M. N. Ollerhead, K. Alfredsen, M. Stickler, A. Harby, M. Robertson, K. D. Clarke, and L. J. LeDrew (2008). A synopsis of 'hydropeaking' studies on the response of juvenile atlantic salmon to experimental flow alteration. $H y$ drobiologia 609(1), 263-275.

Scruton, D. A., C. J. Pennell, M. J. Robertson, L. M. N. Ollerhead, K. D. Clarke, K. Alfredsen, A. Harby, , and R. S. McKinley (2005). Seasonal response of juvenile atlantic salmon to experimental hydropeaking power generation in Newfoundland, Canada. North American Journal of Fisheries Management 25(3), 964-974.

Shawwash, Z. K., T. K. Siu, and S. O. Russell (2000). The B.C. Hydro short term hydro scheduling optimization model. IEEE Transactions on Power Systems 15(3), 1125-1131.

Smokorowski, K. E., R. A. Metcalfe, N. E. Jones, J. Marty, S. Niu, and R. S. Pyrce (2009). Studying ramping rate restrictions. Hydro Review 28(5), 68-88.

Soliman, S. A. and G. S. Christensen (1988). Optimization of the production of hydroelectric power systems with a variable head. Journal of Optimization Theory and Applications 58(2), 301-317.

Statistics Canada (2000). Electric power generating stations. Statistics Canada Catalogue, 57-206-XIB, 1-35.

Svoboda, A. J., C. Tseng, C. Li, and R. B. Johnson (1997). Short-term resource scheduling with ramp constraints. IEEE Transactions on Power Systems 12(1), 77-83.

Thompson, M., M. Davison, and H. Rasmussen (2004). Valuation and optimal operation of electric power plants in competitive markets. Operations Research 52(4), 546-562.

Trebilcock, M. J. and R. Hrab (2005). Electricity restructuring in Ontario. The Energy Journal 26(1), 123-146.

Veselka, T. D., S. Hamilton, and J. McCoy (1995). Optimizing hourly hydro operations at the Salt Lake City area integrated projects. http://www.osti.gov/bridge/servlets/purl/73032-TmMZuC/webviewable/73032.pdf. 
Weron, R. (2008). Market price of risk implied by Asian-style electricity options and futures. Energy Economics 30(3), 1098-1115.

Weron, R., M. Bierbrauer, and S. Truck (2004). Modeling electricity prices: Jump diffusion and regime switching. Physica A 336(1), 39-48.

Wilmott, P. (1998). Derivatives : The Theory and Practice of Financial Engineering. John Wiley \& Sons. 